Florida International University FIU Digital Commons

$11-10-2016$

\title{
Wide-Area Time-Synchronized Closed-Loop Control of Power Systems And Decentralized Active Distribution Networks
}

Mehmet Hazar Cintuglu

Florida International University, mcint015@fiu.edu

DOI: 10.25148 /etd.FIDC001202

Follow this and additional works at: https:// digitalcommons.fiu.edu/etd

Part of the Power and Energy Commons, and the Systems and Communications Commons

\section{Recommended Citation}

Cintuglu, Mehmet Hazar, "Wide-Area Time-Synchronized Closed-Loop Control of Power Systems And Decentralized Active Distribution Networks" (2016). FIU Electronic Theses and Dissertations. 3031.

https://digitalcommons.fiu.edu/etd/3031 


\title{
FLORIDA INTERNATIONAL UNIVERSITY
}

Miami, Florida

WIDE-AREA TIME-SYNCHRONIZED CLOSED-LOOP CONTROL OF POWER SYSTEMS AND DECENTRALIZED ACTIVE DISTRIBUTION NETWORKS

\author{
A dissertation submitted in partial fulfillment of \\ the requirements for the degree of \\ DOCTOR OF PHILOSOPHY \\ in \\ ELECTRICAL ENGINEERING
}

by

Mehmet Hazar Cintuglu 


\section{To: Interim Dean Ranu Jung}

College of Engineering and Computing

This dissertation, written by Mehmet Hazar Cintuglu, and entitled Wide-Area TimeSynchronized Closed-Loop Control of Power Systems and Decentralized Active Distribution Networks, having been approved in respect to style and intellectual content, is referred to you for judgment.

We have read this dissertation and recommend that it be approved.

$\begin{array}{r}\hline \text { Kemal Akkaya } \\ \hline \text { Berrin Tansel } \\ \hline \text { Arif Sarwat } \\ \hline \text { Ismail Guvenc } \\ \hline \text { Mark J. Roberts } \\ \hline \text { Osama Mohammed, Major Professor }\end{array}$

Date of Defense: November 10, 2016

The dissertation of Mehmet Hazar Cintuglu is approved.

Interim Dean Ranu Jung College of Engineering and Computing

Andrés G. Gil Vice President for Research and Economic Development and Dean of the University Graduate School

Florida International University, 2016 
(C) Copyright 2016 by Mehmet Hazar Cintuglu

All rights reserved. 


\section{DEDICATION}

This dissertation is dedicated to my parents, my brother and my girlfriend. Without their support, encouragement, and most importantly their everlasting love, the completion of this work would never have been possible. 


\section{ACKNOWLEDGMENTS}

I would like to express my sincere gratitude to my principal advisor, Professor Osama Mohammed, for his guidance, support, encouragement, and thought-provoking discussions during my doctoral research. This dissertation would not have been possible without his help, support, resolute dedication, and patience. His passion for success has inspired me to come up with new ideas. His expert advice and unsurpassed knowledge of the various fields of electrical energy systems has always provided me an endless supply of idea to move to the next step and complete this dissertation. I am also grateful for the chance he gave me to work under his supervision at his excellent research group at the Energy Systems Research Laboratory. In addition, this atmosphere enabled me to develop

professionally in an environment where research work and engineering ethics are highly respected.

I highly acknowledge the support I received as a research assistant under my major professor research grants received from the Office of Naval Research and the US Department of Energy throughout my research at the energy systems research laboratory. I thank the university graduate school for awarding me the Dissertation Year Fellowship (DYF) which significantly helped me complete this dissertation.

I would like to also thank my dissertation committee members for their insightful comments and constructive suggestions in the review of my dissertation.

Also, I am thankful to all my colleagues in the Energy Systems Research Laboratory. Many graduate and undergraduate student scholars have helped me with discussions, contributions, and assistance contributing to achieving my research goals. I 
also want to mention the support of our departmental staff, especially Ms. Patricia Brammer and Mr. Oscar Silveira, who gave me great help and support.

Finally, I would like to give a special thanks to my mother and father for their unconditional support and love. I also extend my gratitude to for their encouragement and assistance. 


\title{
ABSTRACT OF THE DISSERTATION \\ WIDE-AREA TIME-SYNCHRONIZED CLOSED-LOOP CONTROL OF POWER SYSTEMS AND DECENTRALIZED ACTIVE DISTRIBUTION NETWORKS
}

by

\author{
Mehmet Hazar Cintuglu
}

Florida International University, 2016

Miami, Florida

\section{Professor Osama Mohammed, Major Professor}

The rapidly expanding power system grid infrastructure and the need to reduce the occurrence of major blackouts and prevention or hardening of systems against cyberattacks, have led to increased interest in the improved resilience of the electrical grid. Distributed and decentralized control have been widely applied to computer science research. However, for power system applications, the real-time application of decentralized and distributed control algorithms introduce several challenges.

In this dissertation, new algorithms and methods for decentralized control, protection and energy management of Wide Area Monitoring, Protection and Control (WAMPAC) and the Active Distribution Network (ADN) are developed to improve the resiliency of the power system. To evaluate the findings of this dissertation, a laboratoryscale integrated Wide WAMPAC and ADN control platform was designed and implemented. The developed platform consists of phasor measurement units (PMU), intelligent electronic devices (IED) and programmable logic controllers (PLC). On top of the designed hardware control platform, a multi-agent cyber-physical interoperability 
framework was developed for real-time verification of the developed decentralized and distributed algorithms using local wireless and Internet-based cloud communication.

A novel real-time multiagent system interoperability testbed was developed to enable utility independent private microgrids standardized interoperability framework and define behavioral models for expandability and plug-and-play operation. The state-of-theart power system multiagent framework is improved by providing specific attributes and a deliberative behavior modeling capability. The proposed multi-agent framework is validated in a laboratory based testbed involving developed intelligent electronic device prototypes and actual microgrid setups. Experimental results are demonstrated for both decentralized and distributed control approaches.

A new adaptive real-time protection and remedial action scheme (RAS) method using agent-based distributed communication was developed for autonomous hybrid AC/DC microgrids to increase resiliency and continuous operability after fault conditions. Unlike the conventional consecutive time delay-based overcurrent protection schemes, the developed technique defines a selectivity mechanism considering the RAS of the microgrid after fault instant based on feeder characteristics and the location of the IEDs. The experimental results showed a significant improvement in terms of resiliency of microgrids through protection using agent-based distributed communication. 


\section{TABLE OF CONTENTS}

CHAPTER

PAGE

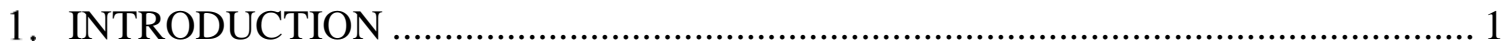

1.1. Introduction to Smart Grids .......................................................................... 1

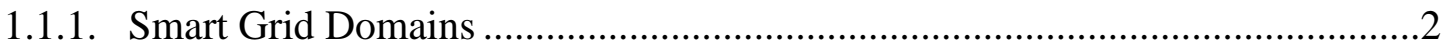

1.1.2. Smart Grid Priority Research Areas .............................................................

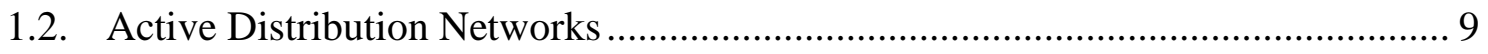

1.2.1. Information and Communication Technology …………...............................10

1.2.2. Decentralized and Distributed Control ...........................................................13

1.2.3. Multi-agent Systems ....................................................................................

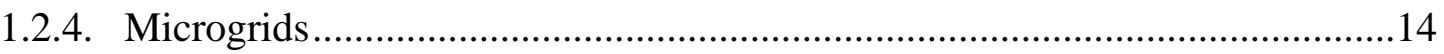

1.3. Wide-Area Monitoring and Control............................................................. 15

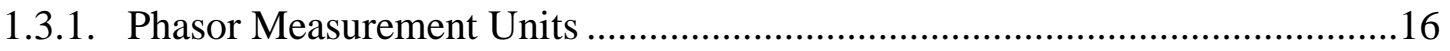

1.3.2. Time Synchronization in Power Systems ……………………….................17

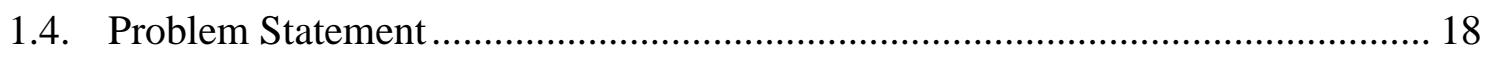

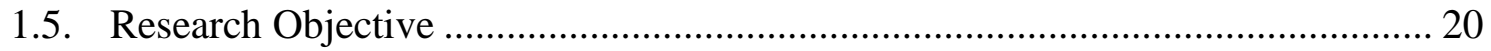

1.6. Original Contributions of the Dissertation........................................................ 24

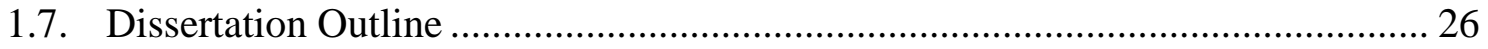

2. DESIGN AND IMPLEMENTATION OF INDUSTRIAL MICROGRID ................ 31

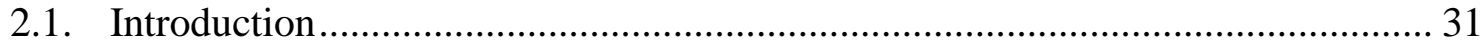

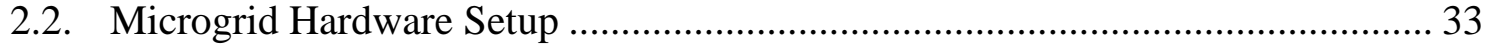

2.2.1. Synchronous Generator..............................................................................

2.2.2. Distribution Lines and Load Models ………………........................................

2.2.3. PV Emulator and Embedded Controller ……………………………..........36

2.2.4. Bi-Directional AC/DC and DC/DC Converters...............................................37

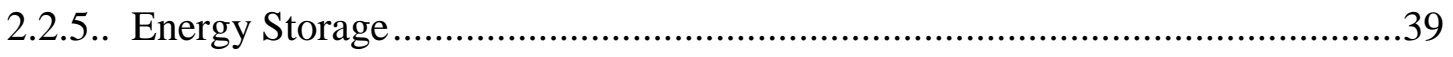

2.3. Microgrid Automation, Protection and Control Setup .......................................... 39

2.3.1. Phasor Measurement Unit.............................................................................. 40

2.3.2. Phasor Data Concentrator ……………………..........................................41 


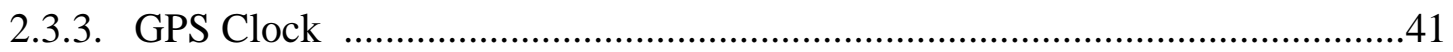

2.3.4. Programmable Logic Controller .................................................................42

2.3.5. Real-Time Automation Controller ………………....................................42

2.3.6. SCADA Interface ...............................................................................43

2.3.7. Grid Connection and Information/Communication Architecture ...................44

3. ISLANDING PERFORMANCE AND CONTROL OF HYBRID MICROGRID .... 46

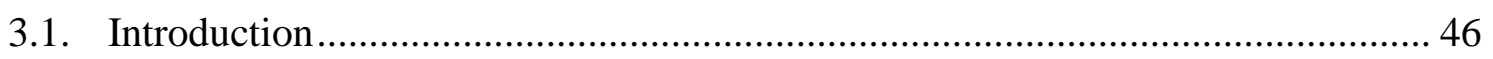

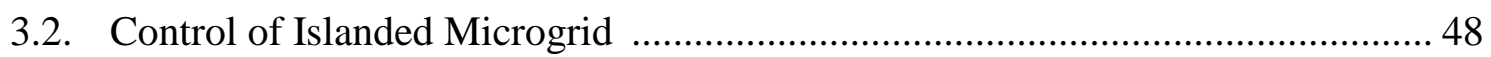

3.3. Operation Mode of Distributed Energy Resources ................................................ 49

3.3.1. Synchronous Generator Grid-Connected Operation .........................................50

3.3.2. Synchronous Generator Islanded Operation ....................................................50

3.3.3. Inverter-based DER Grid-Connected Operation...............................................53

3.3.4. Inverter-based DER Islanded Operation ......................................................56

3.3.5. Inverter-based DER and Synchronous Generator Islanded Operation .............57

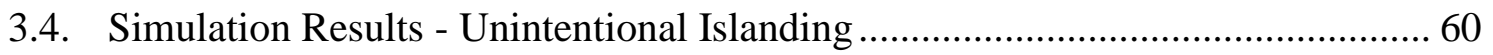

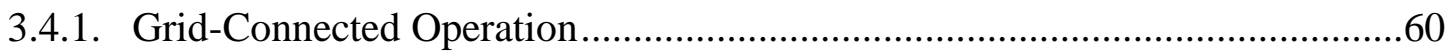

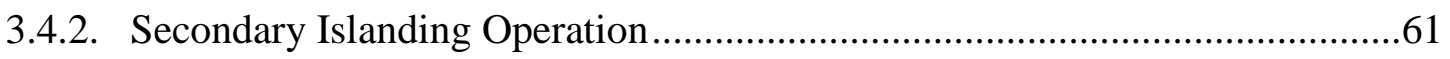

3.4.2. Facility Islanding Operation ......................................................................64

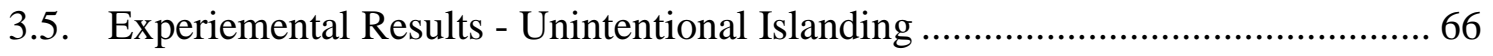

3.5.1. Grid-Connected Operation...........................................................................67

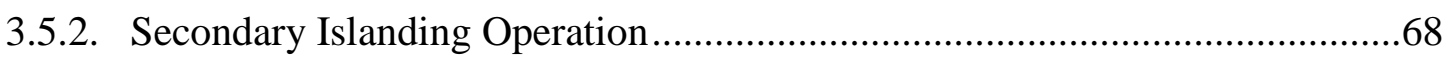

3.5.3. Facility Islanding Operation ...........................................................................69

3.6. Summary of the Results and Conclusion ........................................................... 71

4. SYNCHROPHASOR ASSISTED CONTROL OF HYBRID MICROGRID ............. 72

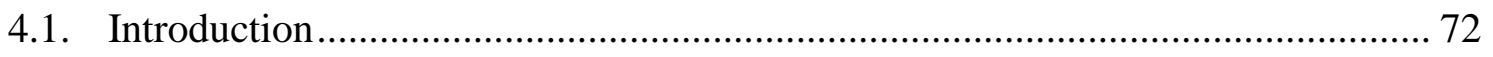

4.2. Synchrophasor Applications in Microgrids ......................................................... 74

4.2.1. Synchrophasor-based Islanding Detection.......................................................74

4.2.2. Resychronization of Microgrids .................................................................76

4.2.3. Protection and Event Analysis .....................................................................77 
4.2.4. DER Scheduling and Ancillary Services ................................................... 79

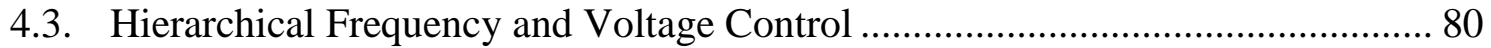

4.4. Performance Monitoring under Pulsed Loads ................................................ 83

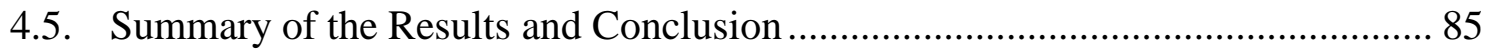

5. PROTECTION OF AUTONOMOUS MICROGRIDS USING AGENT BASED

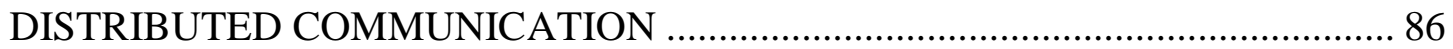

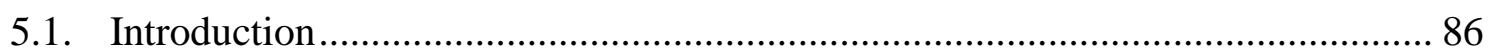

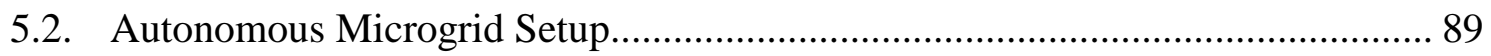

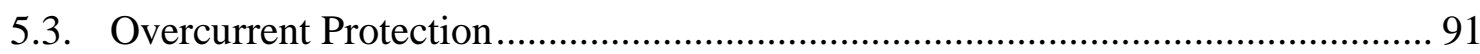

5.3.1. Overcurrent Protection Selectivity Challanges ............................................91

5.3.2. Agent-based Overcurrent Selectivity Method .............................................94

5.3.3. Real-Time Implementation of Overcurrent Protection ...............................100

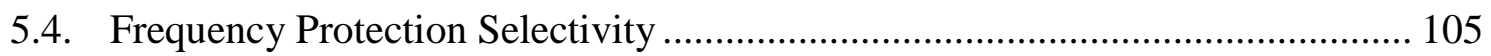

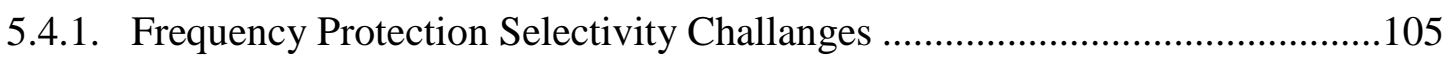

5.4.2. Agent-based Frequency Protection Method..............................................108

5.4.3. Real-Time Implementation of Agent-based Frequency Protection ...............109

5.5. Summary of the Results and Conclusion .................................................... 112

6. FULLY DISTRIBUTED APPROACH FOR AGENT-BASED PROTECTION OF

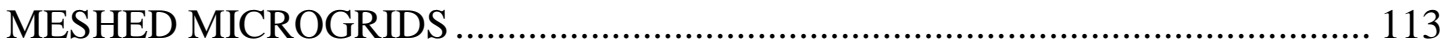

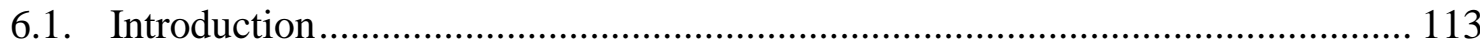

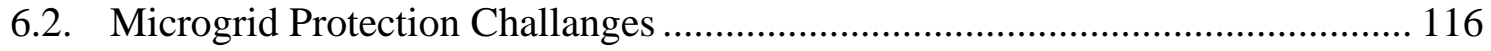

6.2.1. Distributed Microgrid Protection................................................................116

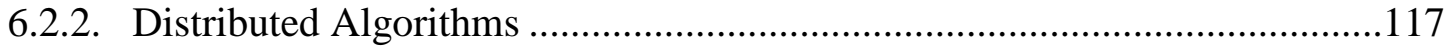

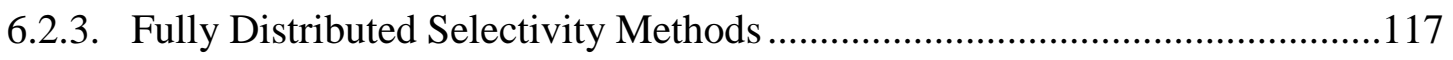

6.3. Implementation of Distributed Algorithms ................................................ 119

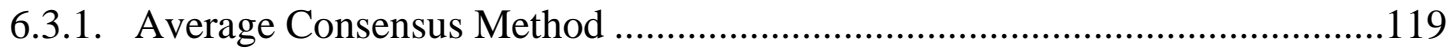

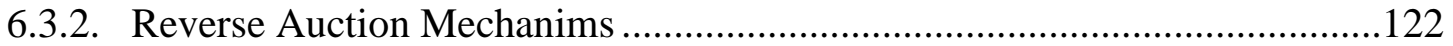

6.3.3. Distributed Depth First Search Algorithm ................................................123

6.4. Simulation and Experimental Results ........................................................... 126

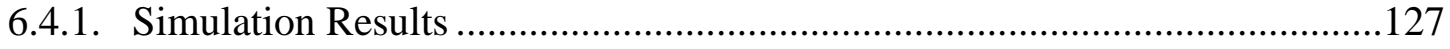


6.4.2. Experimental Results

6.5. Conclusion and Summary of the Results

7. DEVELOPMENT AND APPLICATION OF MICROGRID MULTIAGENT INTEROPERABILITY TEST BED ................................................................. 138

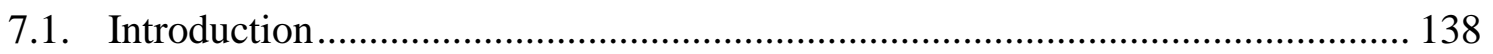

7.2. Hierarchical Microgrid Management............................................................ 142

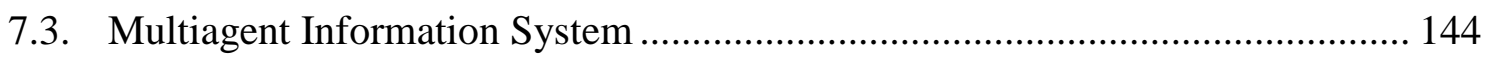

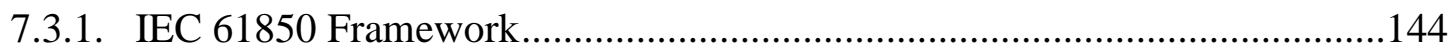

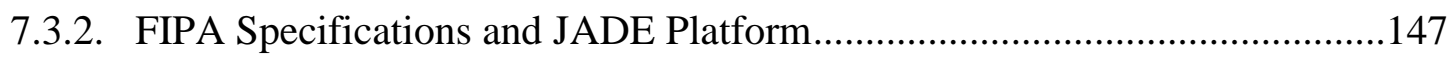

7.3.3. Open Connectivity Unified Architecture ................................................. 148

7.3.4. Developed Prototype Intelligent Electronic Devices ..................................149

7.3.5. Microgrid Management Test Setup ......................................................... 150

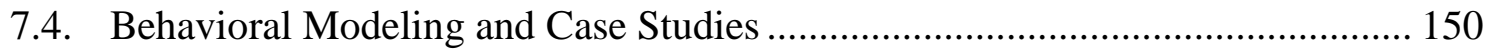

7.4.1. Decentralized Hierarchical Control Case Studies .......................................151

7.4.2. Distributed Control Consensus-based Economic Dispatch Case Study ........158

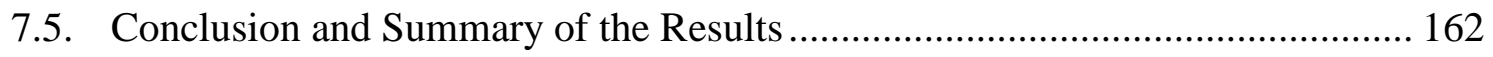

\section{DEVELOPMENT OF CLOUD COMMUNICATION FOR REMOTE ACCESS}

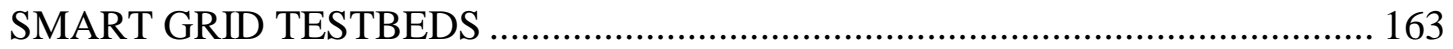

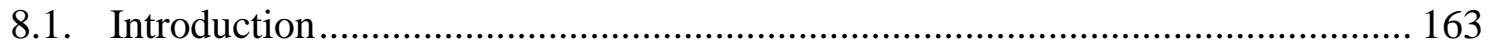

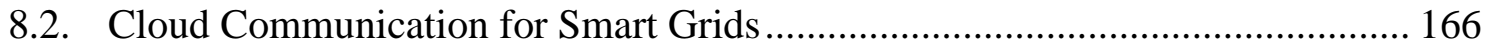

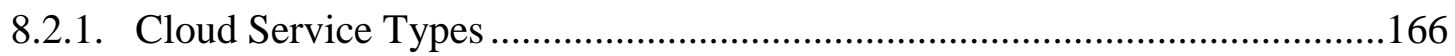

8.2.2. Cloud-based Emerging Power System Applications ...................................166

8.2.3. Security, Privacy and Delay Challanges ..................................................166

8.3. Smart Grid Test Bed Implementation ........................................................ 168

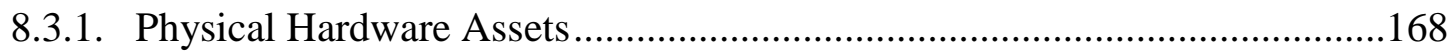

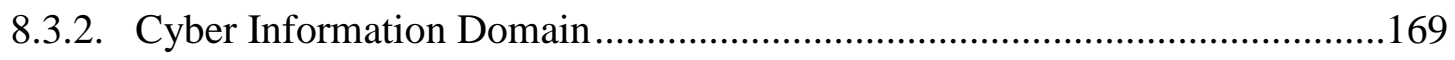

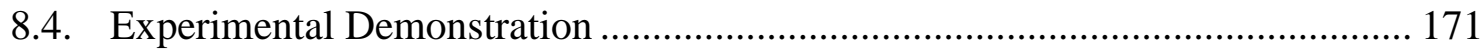

8.5. Conclusion and Summary of the Results ................................................. 174

9. MULTIAGENT-BASED FULLY DISTRIBUTED MICROGRID CONTROL AND COMMUNICATION ARCHITECTURE ............................................. 175 


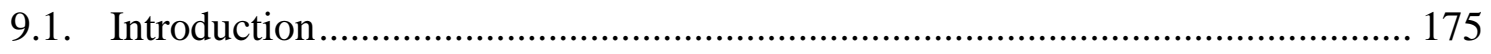

9.2. Wireless and Cloud Communication Architecture ............................................ 177

9.2.1. Hybrid Wireless Mesh Network Protocol.....................................................177

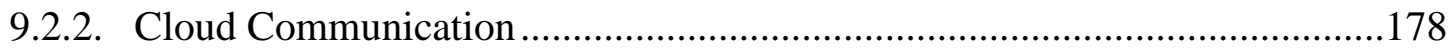

9.3. Multiagent System and Control Hierarchy ………………………................... 179

9.3.1. Microgrid Control Hierarchy …………………..........................................

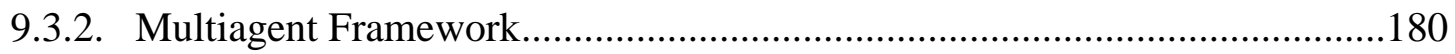

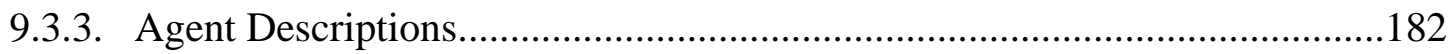

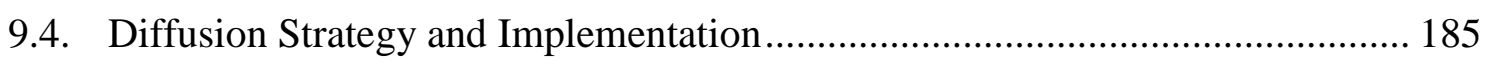

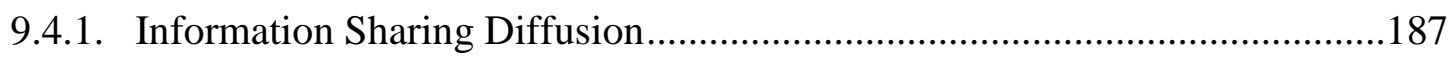

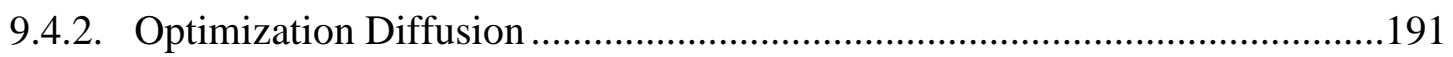

9.4.3. Modified Optimization Diffusion ................................................................193

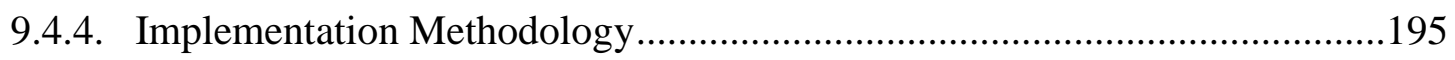

9.5. Simulation and Experimental Results ........................................................... 198

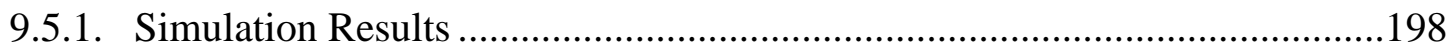

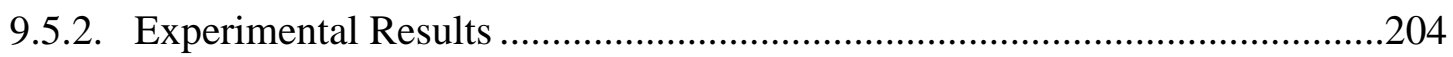

9.6. Conclusion and Summary of the Results ......................................................... 206

10. MULTIAGENT-BASED GAME THEORETIC REVERSE AUCTION MODEL

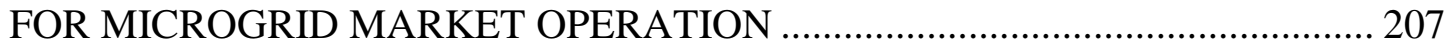

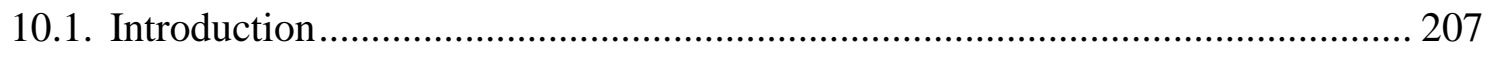

10.2. Game Theoretic Reverse Auction Model ........................................................ 210

10.3. Microgrid Agent Modeling for Market Operation.............................................. 212

10.4. Hardware Setup for Market Agents Implementation........................................... 218

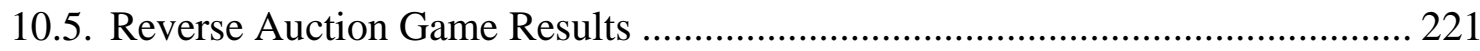

10.6. Conclusion and Summary of the Results ......................................................... 228

\section{BEHAVIOR MODELING AND AUCTION ARCHITECTURE OF} NETWORKED MICROGRIDS FOR WIDE AREA FREQUENCY SUPPORT ... 229

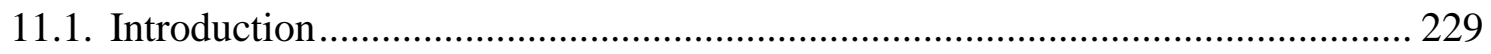

11.2. Automatic Generation Control for Intermittent Generation Profile .................. 231

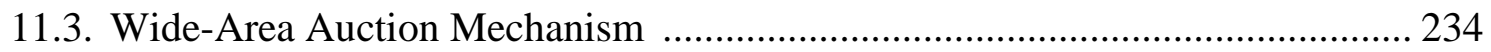

11.3.1. Reserved Price Selection for Aggregator Agent ............................................234 
11.3.2. Reserved Price Selection for Microgrid Agents

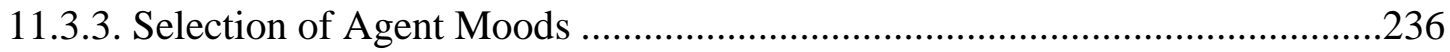

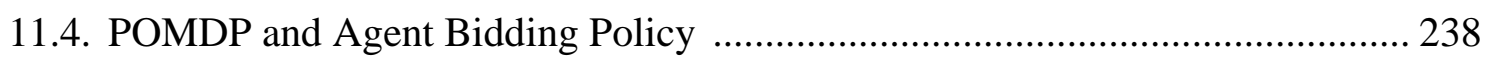

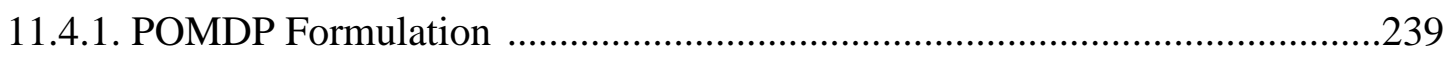

11.4.2. Short Term Policy Determination ........................................................241

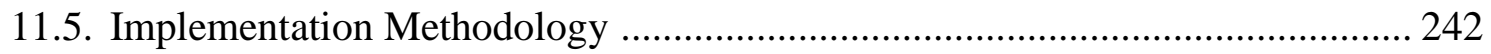

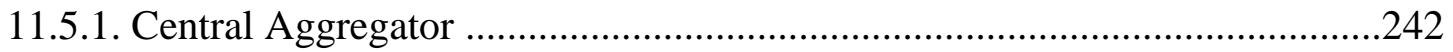

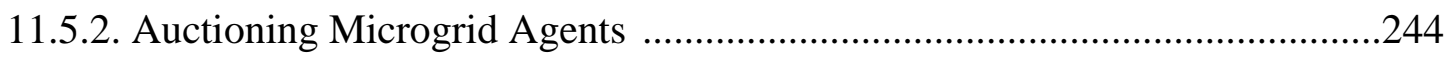

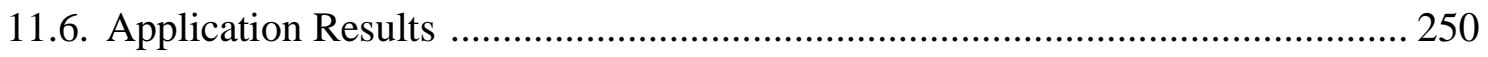

11.6.1. Cyber-Physical Multiagent Framework ................................................250

11.6.2. Real-Time Experiemental Demonstration ...........................................252

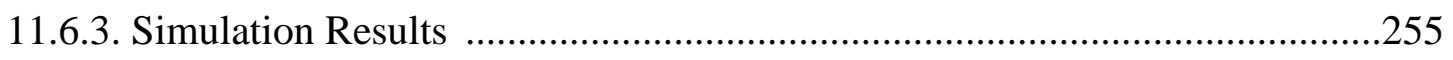

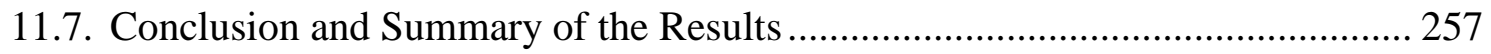

12. CONCLUSIONS AND RECOMMENDATIONS FOR FUTURE WORK............ 259

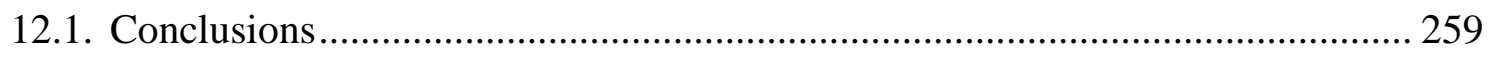

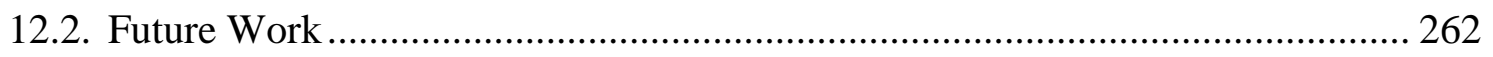

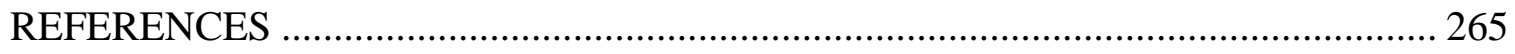

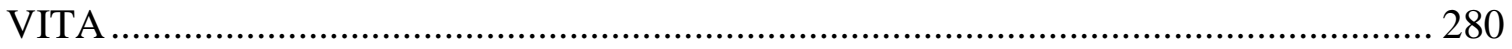




\section{LIST OF TABLES}

TABLE

PAGE

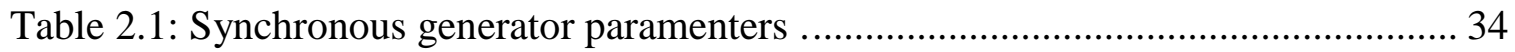

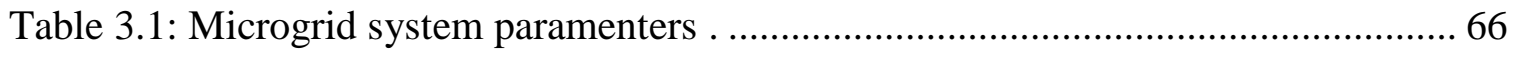

Table 5.1: Fault currents for grid-connected operation . ........................................ 93

Table 5.2: Definite time-delay characteristics ..................................................... 95

Table 6.1: Consensus-based auction fault clearing algorithm ................................. 123

Table 6.2: Distributed depth-first search node discovery algorithm ........................... 125

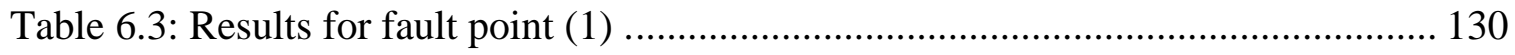

Table 9.1: Diffusion vs consensus convergence comparison ................................... 190

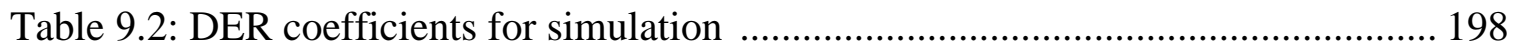

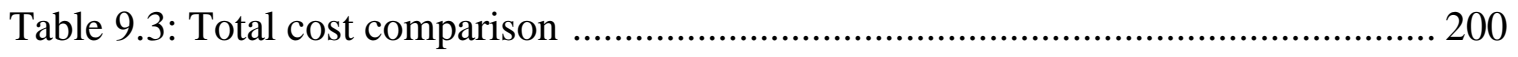

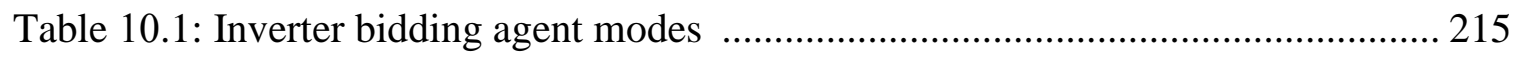

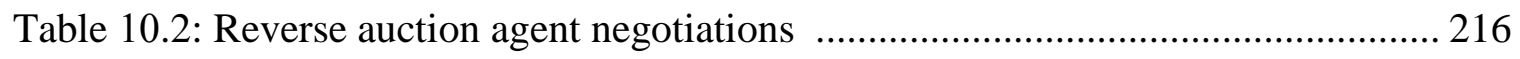

Table 10.3: One hour random game output .......................................................... 223

Table 11.1: Behavioral response-based auction algorithm .................................... 243

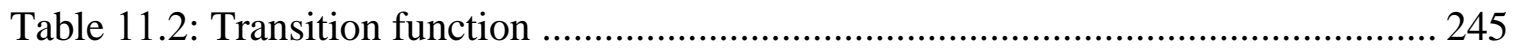

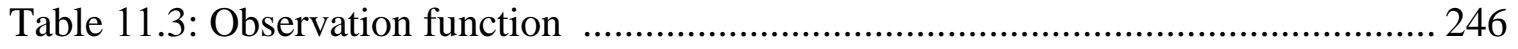

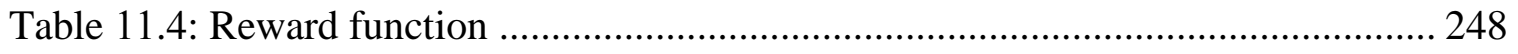

Table 11.5: 3 -step conditional plan outcome .................................................... 248

Table 11.6: Reverse auction result ................................................................... 254

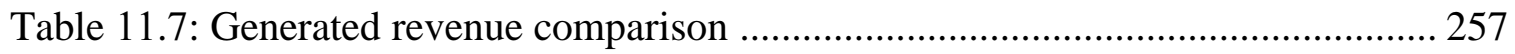




\section{LIST OF FIGURES}

FIGURE

PAGE

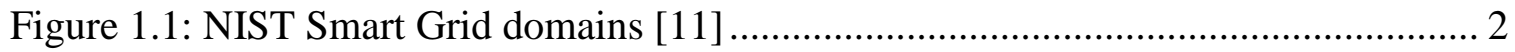

Figure 1.2: Active distribution network overview ............................................... 10

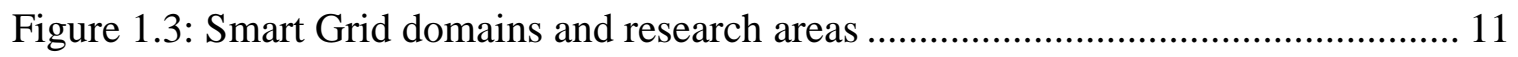

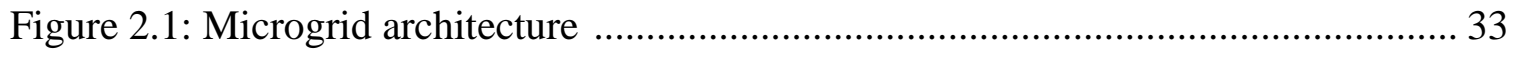

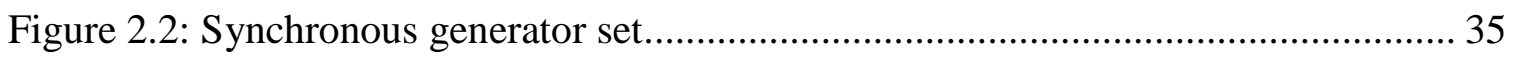

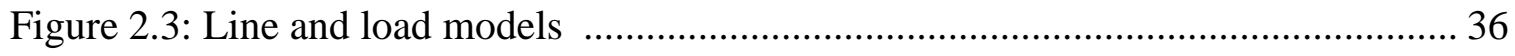

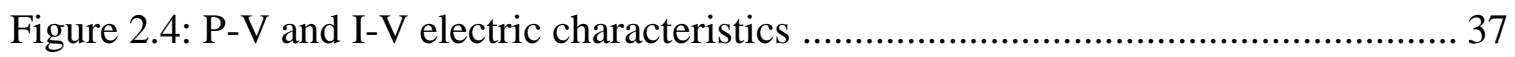

Figure 2.5: DSpace embedded controller and PV emulator ....................................... 37

Figure 2.6: (a) AC/DC converter; (b) DC/DC converter; (c) battery bank ..................... 39

Figure 2.7: Microgrid automation architecture .................................................... 40

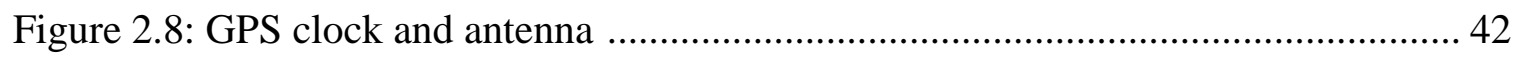

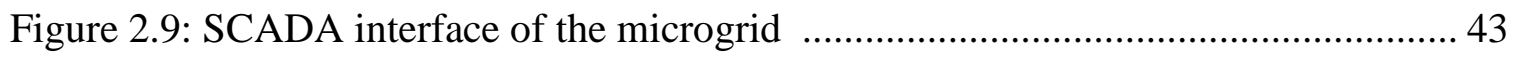

Figure 3.1: Microgrid architecture scheme .......................................................... 47

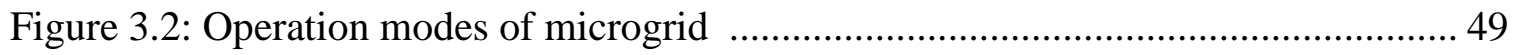

Figure 3.3: Synchronous generator grid connected (P-V) operation ............................ 50

Figure 3.4: Synchronous generator islanded operation .......................................... 51

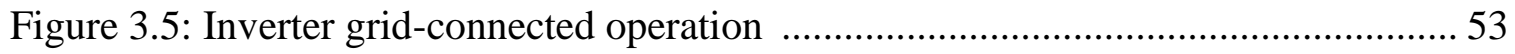

Figure 3.6: Control block for bi-directional DC/DC converter ................................. 55

Figure 3.7: Detailed inverter grid connected operation ........................................ 55

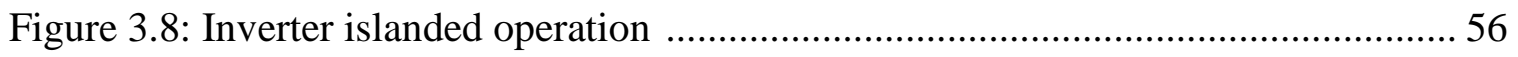

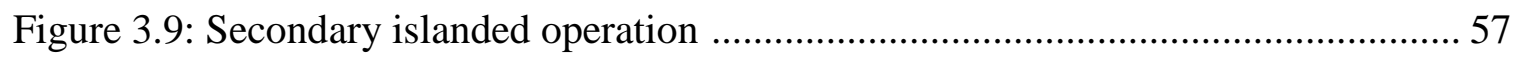

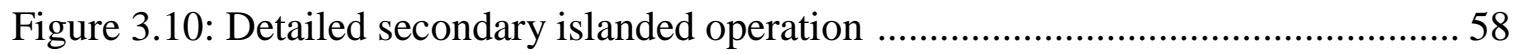




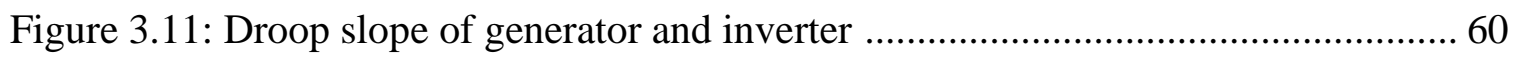

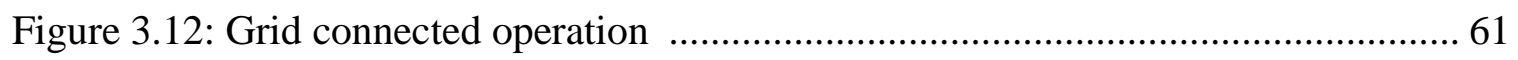

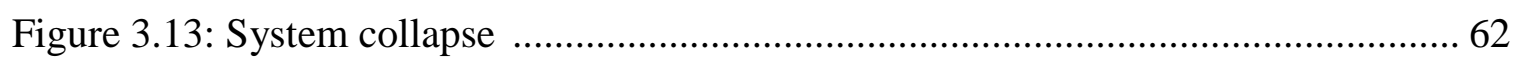

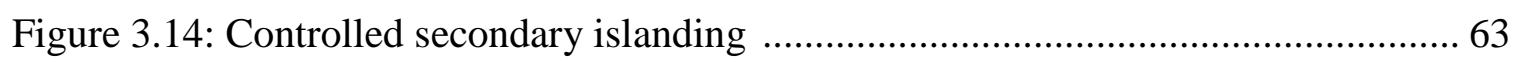

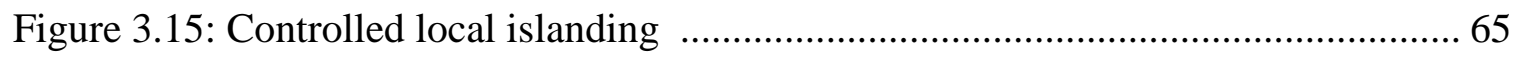

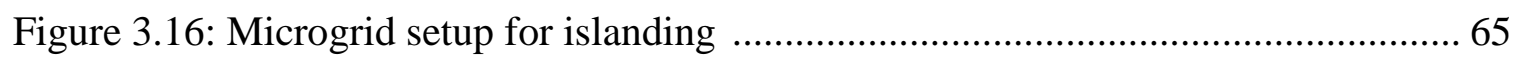

Figure 3.17: Synchronous generator grid-connected operation in experiment ............... 67

Figure 3.18: Inverter-based DER grid-connected operation in experiement ................... 67

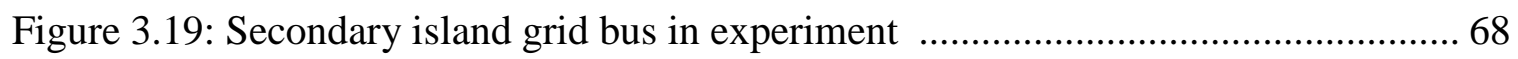

Figure 3.20: Secondary islanding inverter-based DER bus in experiment ..................... 69

Figure 3.21: Facility islanding of synchronous generator in experiment $\ldots \ldots \ldots \ldots \ldots \ldots \ldots \ldots . . . . . . . . . .70$

Figure 3.22: Facility islanding of inverter-based DER in experiment .......................... 71

Figure 4.1: Islanding detection function blocks .................................................. 75

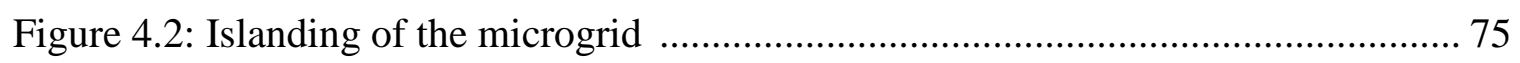

Figure 4.3: Resynchronization of the microgrid ................................................. 76

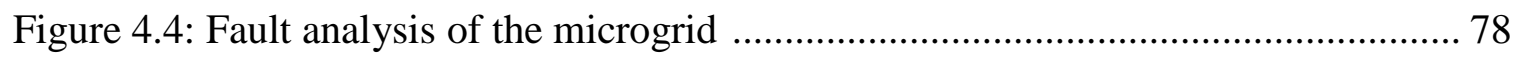

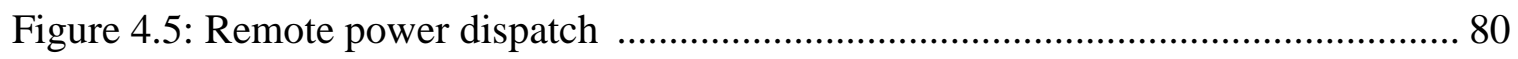

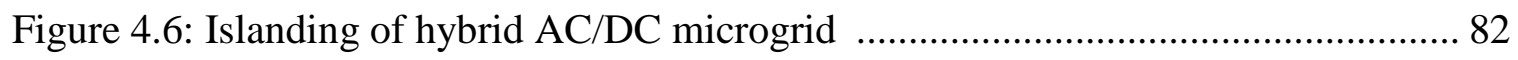

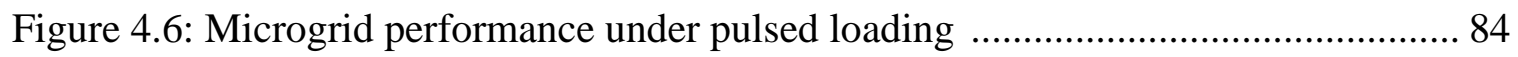

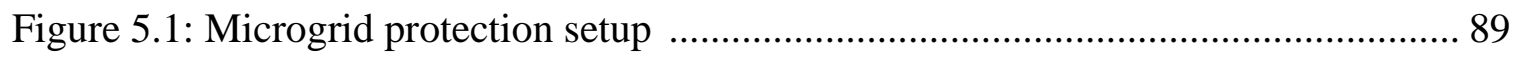

Figure 5.2: IED deployment on microgrid feeders and the related logical nodes .......... 90

Figure 5.3: Fault current routes for different fault points ....................................... 92

Figure 5.4: Publisher / subscriber pattern and GOOSE message traffic ........................ 96

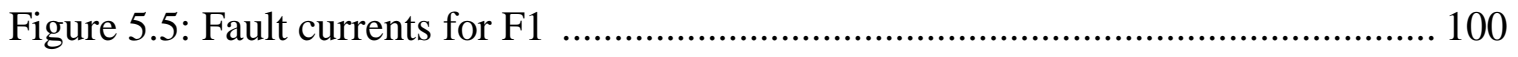

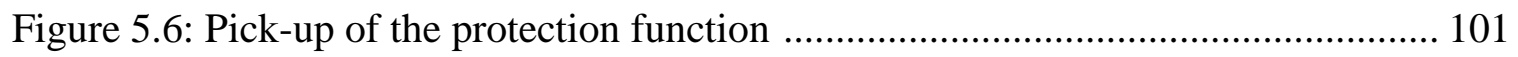


Figure 5.7: Blocked circuit breaker during IED4 pick-up ..................................... 102

Figure 5.8: Circuit breaker status and active setting change .................................... 103

Figure 5.9: Microgrid frequency recovery after islanding ......................................... 104

Figure 5.10: Voltage, active and reactive power change during islanding ................... 104

Figure 5.11: Operation modes of autonomous microgrids ....................................... 105

Figure 5.12: Power demand under pulsed load .................................................... 110

Figure 5.13: Microgrid frequency under pulse load train ........................................ 111

Figure 5.14: Phase angle difference between microgrid buses ................................... 111

Figure 5.15: Blocked frequency protection and load shedding operation ................... 112

Figure 6.1: Meshed microgrid architecture ........................................................ 120

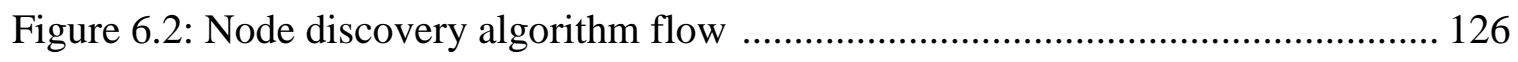

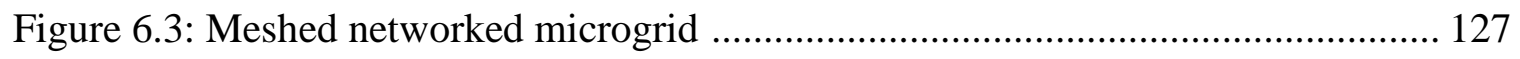

Figure 6.4: Fault currents in each node for three different fault impedence ................. 128

Figure 6.5: First average consensus result ........................................................... 129

Figure 6.6: Second round average consensus result ............................................ 129

Figure 6.7: Consensus on IEEE 14 bus network topology ....................................... 131

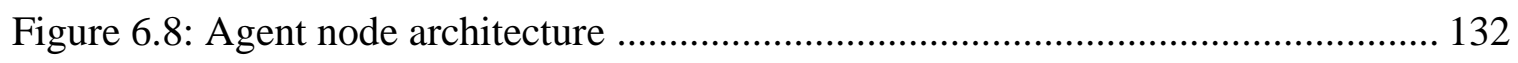

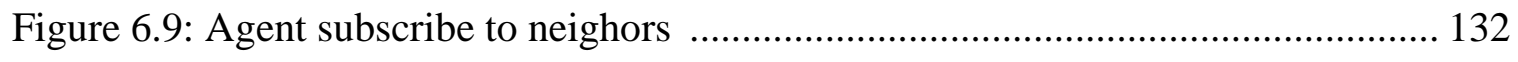

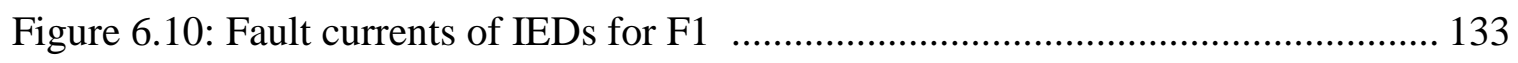

Figure 6.11: Relay1 convergence at 232nd iteration and message content .................. 134

Figure 6.12: Relay1 communication with adjacent agents ..................................... 134

Figure 6.13: Relay1 agent initializes DDFS algorithm ........................................ 135

Figure 7.1: Network types for microgrid control .................................................... 139

Figure 7.2: Hierarchical control of a microgrid ................................................ 142

Figure 7.3: Cyber-physical infrastrcutre at FIU Smart Grid test bed .......................... 145 
Figure 7.4: Object name of a circuit breaker position value 146

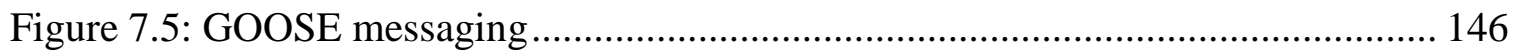

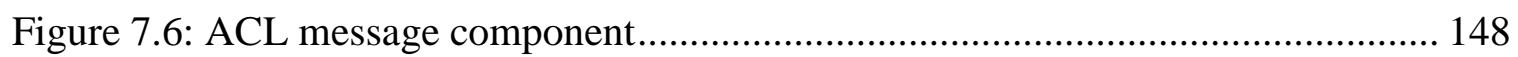

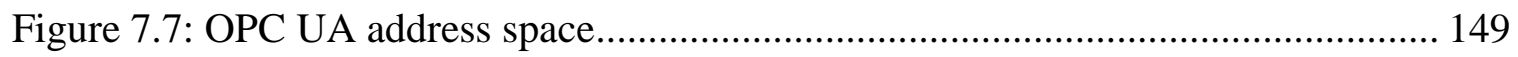

Figure 7.8: Case study flow chart ……………………........................................... 151

Figure 7.9: Implemented agent behaviors.............................................................. 152

Figure 7.10: Tertiary control process ………………………………………….... 155

Figure 7.11: Secondary control process …………………........................................ 157

Figure 7.12: Graph representation of the microgrid network ....................................... 159

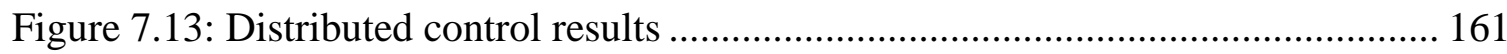

Figure 8.1: Cyber-physical infrastructure and cloud communication setup ................... 168

Figure 8.2: Overcurrent protection one-line diagram ..................................................... 171

Figure 8.3: Overcurrent protection experimental results .............................................. 173

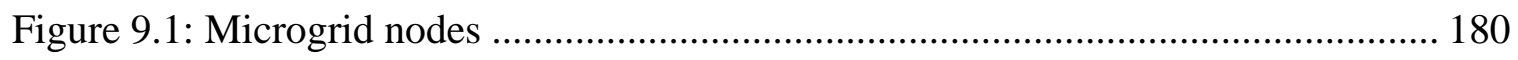

Figure 9.2: Agent communication network ………………................................... 181

Figure 9.3: Energy storage system marginal cost function.......................................... 184

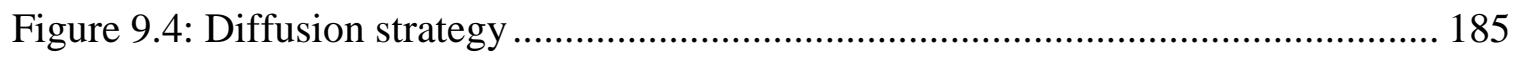

Figure 9.5: Consensus vs diffusion information sharing (9-Node)................................ 189

Figure 9.6: Consensus vs diffusion information sharing (73-Node)............................... 191

Figure 9.7: Nudge methodology ............................................................................. 194

Figure 9.8: Droop no-load frequency adjustments ...................................................... 196

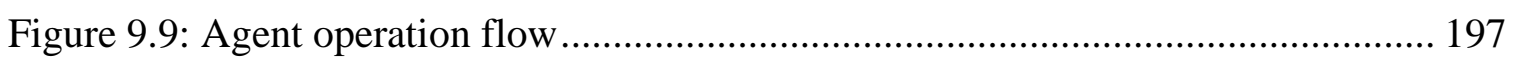

Figure 9.10: Information sharing performance comparison ........................................... 199

Figure 9.11: Cost optimization comparison (centralized vs diffusion)........................... 200

Figure 9.12: Microgrid islanding power output and battery state of charge................... 201 


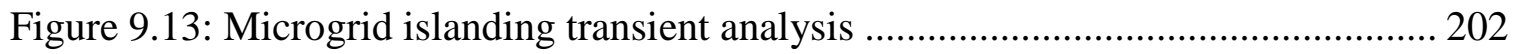

Figure 9.14: Battery energy storage system no-load frequency adjustments ................ 203

Figure 9.15: Experimental islanding - distributed optimization performance .............. 205

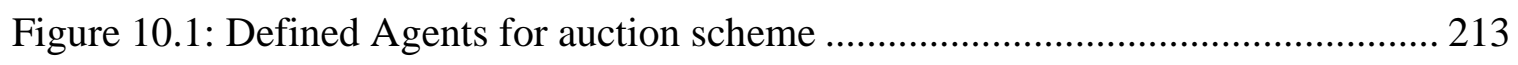

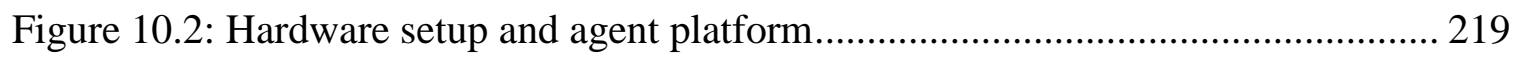

Figure 10.3: Auctioning agents hardware setup .................................................. 220

Figure 10.4: Auction process in 16th hour............................................................... 224

Figure 10.5: Sniffer agent: Aggregator agent interaction with other bidder agents ....... 225

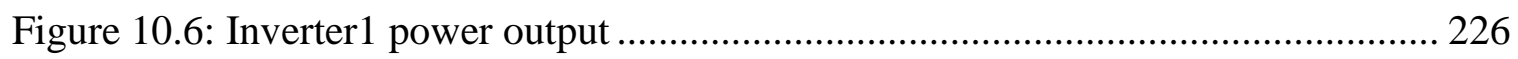

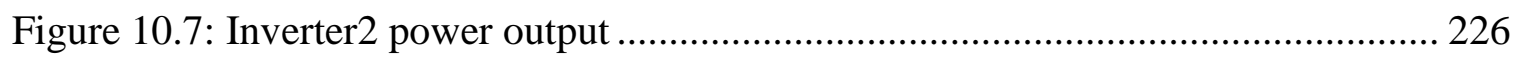

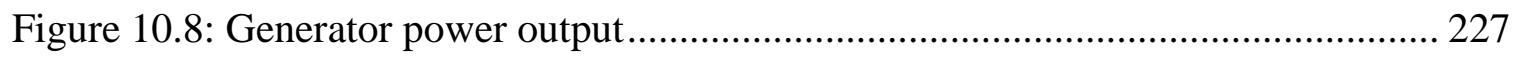

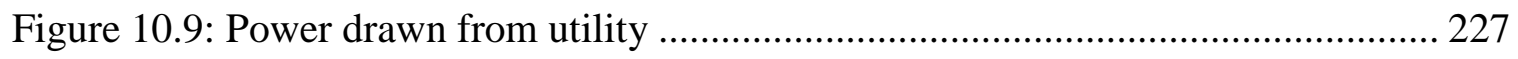

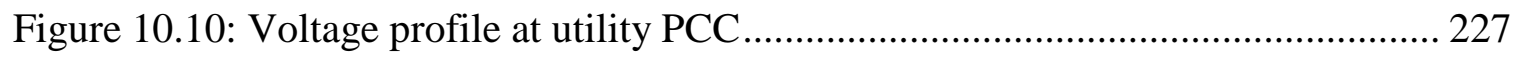

Figure 11.1: Two-area power system with microgrid penetration .............................. 232

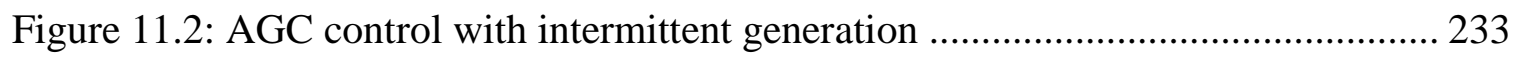

Figure 11.3: Belief monitoring in policy execution for MG1 .................................... 249

Figure 11.4: Agent node architecture.............................................................. 251

Figure 11.5: Real-time experiment of AGC and auction scheme .............................. 252

Figure 11.6: Correspondence between central aggreagtor and microgrid agent ........... 253

Figure 11.7: Simulation results of AGC and auction scheme.................................. 256

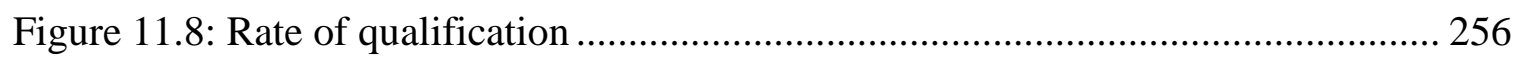




\section{LIST OF ACRONYMS}

\begin{tabular}{|c|c|}
\hline NIST & National Institute of Standards and Technology \\
\hline DER & Distributed Energy Resource \\
\hline IED & Intelligent Electronic Device \\
\hline EMS & Energy Management System \\
\hline DMS & Distribution Management System \\
\hline RTO & Regional Transmission Operator \\
\hline ISO & Independent System Operator \\
\hline WAMPAC & Wide Area Monitoring Protection and Control \\
\hline $\mathrm{ADN}$ & Active Distribution Network \\
\hline PMU & Phasor Measurement Unit \\
\hline DR & Demand Response \\
\hline RAS & Remedial Action Scheme \\
\hline $\mathrm{AGC}$ & Automatic Generation Control \\
\hline POMDP & Partially Observable Markov Decision Process \\
\hline DSO & Distribution System Operator \\
\hline PEV & Plug-in Electric Vehicle \\
\hline AMI & Advanced Metering Infrastructure \\
\hline VAR & Volt-Ampere-Reactive \\
\hline DNP & Distributed Network Protocol \\
\hline HAN & Home Area Network \\
\hline M2M & Machine-to-Machine \\
\hline LTE & Long Term Evolution \\
\hline
\end{tabular}




$\begin{array}{ll}\text { SDN } & \text { Software Defined Network } \\ \text { PLC } & \text { Programmable Logic Controller } \\ \text { GOOSE } & \text { Generic Object Oriented Substation Event } \\ \text { ROCOF } & \text { Rate of Change of Frequency } \\ \text { FE } & \text { Frequency Error } \\ \text { TVE } & \text { Total Vector Error } \\ \text { PDC } & \text { Phasor Data Concentrator } \\ \text { UTC } & \text { Coordinated Universal Time } \\ \text { DG } & \text { Distributed Generation } \\ \text { RTAC } & \text { Real-Time Automation Controller } \\ \text { OPC UA } & \text { Open Connectivity Unified Architecture } \\ \text { FIPA } & \text { Foundation of Intelligent Physical Agents } \\ \text { IOT } & \text { Internet of Things } \\ \text { HWMP } & \text { Hybrid Wireless Mesh Protocol } \\ \text { ACL } & \text { Agent Communication Language } \\ \text { DDFS } & \text { Distributed Depth First Search } \\ \text { UMTS } & \text { Universal Mobile Telecommunication System } \\ \text { DSL } & \text { Digital Subscriber Line } \\ \text { SCADA } & \text { Supervisory Control and Data Acquisition } \\ \text { LED } & \text { Light Emitting Diode } \\ \text { MPPT } & \text { Maximum Power Point Tracking } \\ \text { PCC } & \text { Point of Common Coupling } \\ \text { Digital Signal Processor } \\ \text { HSP }\end{array}$




$\begin{array}{ll}\text { IGBT } & \text { Insulated Gate Bipolar Transistor } \\ \text { UTC } & \text { Coordinated Universal Time } \\ \text { SEL } & \text { Schweitzer Engineering Laboratory } \\ \text { HMI } & \text { Human Machine Interface } \\ \text { RES } & \text { Renewable Energy System } \\ \text { PI } & \text { Proportional Integral } \\ \text { VSI } & \text { Voltage Source Inverter } \\ \text { PLL } & \text { Phase Locked Loop } \\ \text { RTU } & \text { Remote Terminal Unit } \\ \text { MG } & \text { Micro Grid } \\ \text { ACE } & \text { Area Control Error } \\ \text { DT } & \text { Definite Time Delay } \\ \text { VSR } & \text { Vector Surge Relay } \\ \text { DF } & \text { Directory Facilitator }\end{array}$




\section{INTRODUCTION}

\subsection{Introduction to Smart Grids}

The existing power grid was not designed in a flexible way to meet future widearea control and decentralized active distribution network demands to support advanced monitoring, integration of renewable energy resources, adaptive protection, and deregulated operation of power markets. The emerging involvement of "smart" devices, communication, and management mechanisms are promising [1]. However, the existing power grid cannot provide satisfactory solutions to extensive demands with its current state. Therefore, the power grid infrastructure is being restructured as a cyber-physical system including smart devices not only to carry power flow, but also to transmit data for advanced monitoring and control applications [2],[3],[4],[5],[6]. Enhancement of the power grid using two-way flows of electricity and information is expected to form the smart grids equipped with intelligent features such as self-healing [10], adaptive protection and control, customer involvement [8],[9], and electric vehicles [7]. In order to stimulate smart grid realization, National Institute of Standards and Technology (NIST) has defined interconnected domains and priority research areas [11]. In this chapter, the commonly agreed smart grid domains and on-going research efforts for smart grids are presented. This chapter also specifically discusses the role of wide-area control and decentralized control of active distribution networks in smart grid environment. 


\subsubsection{Smart Grid Domains}

The smart grid is composed of seven interconnected domains according to NIST as shown in Figure 1.1. Each domain and its sub-domains include corresponding devices, systems, or programs. The devices can be smart meters, distributed energy resources (DER), or intelligent electronic devices (IED). Various systems composed of these devices establish decision-making and information exchange between domains with intended applications. Individual domains have to be evaluated separately; however, there are common requirements such as communication protocols, communication media, networking, and security.

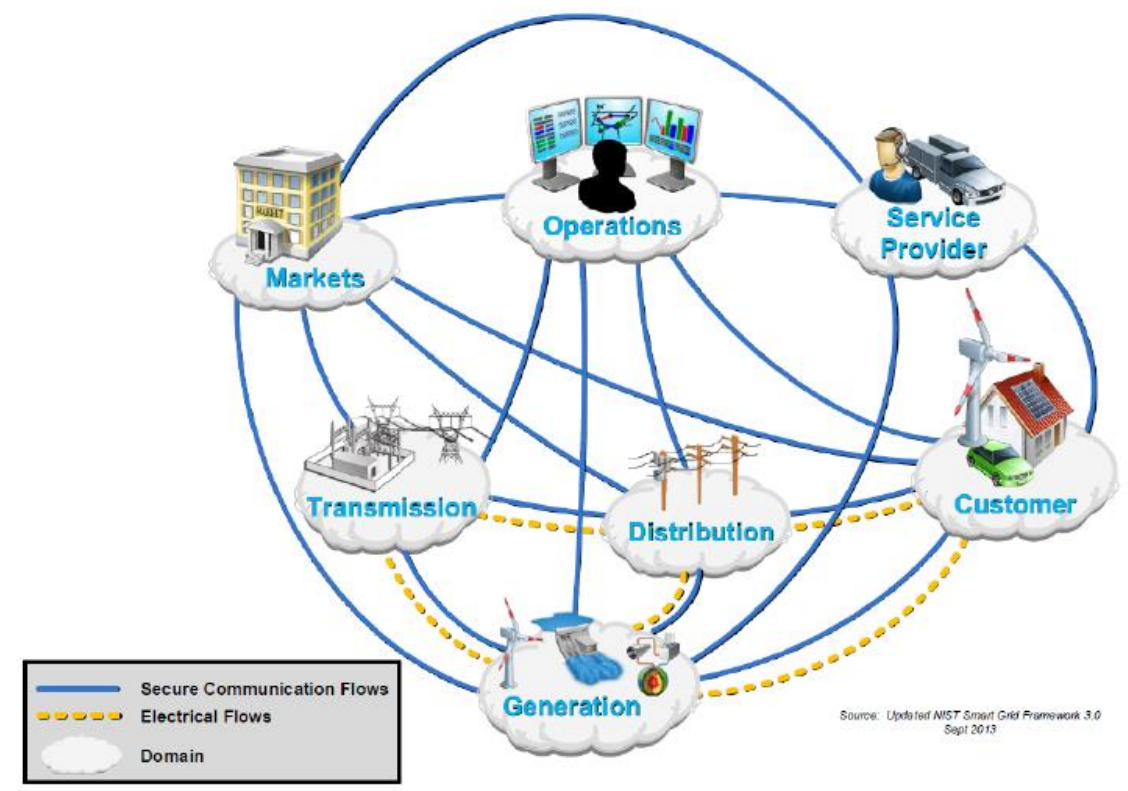

Figure 1.1: NIST Smart Grid Domains [11].

\subsubsection{Customer Domain}

In the customer domain, three types of end users exist: home, commercial/building, and industrial. With the emerging smart grid, end users are also in an ongoing change from 
consumers to producer-consumers (prosumers) by providing DER, storage, and interactive energy management. The main interaction of the prosumers and grid operators is realized through microgrid management [12], [13]. Microgrids are the small scale decentralized electricity networks featuring internal generation and distribution with individual owner priorities. Prosumers are expected to be equipped with a cyber-physical infrastructure and be aware of the consequences of their actions on the power grid.

\subsubsection{Market Domain}

Market management, retailing, aggregation, trading, economical unit commitment, scheduling of DERs, and power sharing between multiple units in a microgrid are the major concerns of this domain [14]. In open market conditions, utilities are no longer monopolized, and DER stakeholders are the private entities that can compete with regional utilities. The electricity trade can be handled locally in a region by several microgrid investors or cross-border trading by utility wholesale market and neighboring regional systems. A vast number of DERs should be able to operate incorporating a large number of complicated operational functions [15]. New generation grids require fast intelligent decision-making algorithms and advanced cyber-physical infrastructure since the power system operators will be inefficient in dealing with highly active and changing operations in the future grid.

\subsubsection{Service Provider Domain}

The dynamic market-driven ecosystem system is the major concern of the service providers, while ensuring a safe operation of the critical power structure. Customer management, smart device installment, and smart building management which can respond 
to demand response signals and billing facilities are the typical applications in the service provider domain [16], [17].

\subsubsection{Operations Domain}

The operation domain is the entity responsible for safe and reliable operation of the power system. Energy management systems (EMS) handle the efficient operation of the transmission level operations, while distribution management systems (DMS) are utilized to handle distribution level. The applications of this domain includes extensive power system operations such as monitoring, control, protection, and analysis [18].

\subsubsection{Bulk Domain}

Traditional large scale generation units such as nuclear, thermal, hydro plants, wind farms, and large scale solar generation are considered as bulk generation units. The generated power is delivered through transmission lines, thus the interaction with the transmission domain is the most critical interface for this domain.

\subsubsection{Transmission Domain}

Regional Transmission Operators or Independent System Operators (RTO/ISO) are the responsible entities for safe operation of the transmission domain. The generated power from bulk generation units is safely transferred to the distribution domain by the transmission domain with the help of generation (supply) and load (demand) balancing practices. Substations are the core components of the transmission domain where high voltage is reduced to distribution level across the electric supply chain. 


\subsubsection{Distribution Domain}

The distribution domain serves the interconnection between transmission domains and the customer domain. Metering points, loads, DERs and microgrids are the components of the distribution domain.

\subsubsection{Smart Grid Priority Research Areas}

Smart grid research is prioritized on nine key functionalities: wide-area situational awareness, demand response and consumer energy efficiency, distributed energy resources, energy storage, electric transportation, advanced metering infrastructure, distribution grid management, and cybersecurity and network communications [11]. A cyber-physical system, including cyber security and network communications is a common necessity for all key functionalities.

\subsubsection{Wide-Area Situational Awareness}

A major blackout, such as the one occurred August 14, 2003 in North American Eastern Interconnection, can result wide scale power loss for millions of customers [15]. In order to avoid such devastating disasters, monitoring and display of the power system components is required across the interconnection over large geographic areas in near realtime. The aim of the situational awareness research is to diagnose, anticipate, and respond to prevent problems before disruptions arise. A safe, reliable, and economical electric power system requires advanced WAMPAC capabilities and applications. PMUs are the devices that provide time-synchronized coherent data from the entire network, which enables the complete system status to be observable and controllable in real-time. Traditionally, most of the efforts have been shown for WAMPAC systems in transmission networks for various applications, including transient stability, modal estimation, and load 
shedding; and more recently, it has been realized that PMUs represent a useful contribution for the challenging operation of ADNs.

\subsubsection{Demand Response and Consumer Energy Efficiency}

Demand response (DR) in deregulated electricity markets provide a mechanism and incentives for utilities, business, industrial, and residential customers to lower energy use during times of peak demand or when the power reliability is at risk [19]. Reliable operation of the electricity system necessitates a perfect balance between supply and demand in realtime, where DR is crucial for optimizing the balance of the power and supply through smart loads with an advanced communication infrastructure such as a meshed network, cellular, cloud or web-based energy information system.

\subsubsection{Distributed Energy Resources}

DER research area covers utility-independent generation units (non-bulk) and energy storage behind the prosumer energy meter. The generated power is mainly consumed on the prosumer premise as a negative load [20]. Although it is not favorable to distribution system operators (DSO), in some cases, reverse power might be drawn from the prosumer side. Advanced aggregated DERs would form independent grid architectures incorporating microgrids, which can be isolated from the grid in case of a utility outage to form a more resilient and reliable system.

\subsubsection{Energy Storage}

The energy storage concept covers conversion of electrical energy from a power network into a form of energy which can be stored and converted back to electrical energy [21]. Electricity storage research includes many physical forms and is thus managed by various interdisciplinary engineering relationships such as pumped hydroelectric, 
compressed gas, flywheel (mechanic), battery, and super capacitor. New storage capabilities - especially for distributed storage would benefit the entire grid, from generation to end use [22].

\subsubsection{Electric Transportation}

Economic and environmental incentives reshape the traditional transportation scheme by enabling large-scale integration of plug-in electric vehicles (PEV), which can significantly reduce dependence on oil and dramatically reduce the carbon footprint [23], [24]. The research on electric transportation covers PEV battery banks, wired-wireless charging stations, and large-scale grid integration.

\subsubsection{Advanced Metering Infrastructure}

Advanced metering infrastructure (AMI) technology is an integration of technologies that provide an intelligent connection between consumers and system operators. System operators implement a residential demand response and price signaling mechanism to serve according to dynamic pricing [25]. It consists of communications between hardware and software with advanced local communication capabilities (e.g. Bluetooth and ZigBee protocols) [26] and Internet communications [27] (e.g. Wi-Fi, digital subscriber line (DSL), and universal mobile telecommunication system (UMTS).

\subsubsection{Distribution Grid Management}

Distribution grid management focuses on active distribution operations including Volt-VAR optimization/control, conservation voltage reduction, power quality improvements, system reliability improvements, and outage management. Advanced cyber-physical architectures on distribution grid management aims to maximize the 
performance of feeders, transformers, and other components of the networked distribution systems and to integrate with transmission systems and customer operations.

\subsubsection{Cyber Security}

The development of smart grid solutions are heavily dependent on power system communication and information infrastructures [28], [29]. While total network and computer integration boost power system capability, vulnerabilities to cyber-attack threats drastically increases. Existing cyber security solutions may not be favorable or efficient for smart grid cyber-physical system security concerns, but require domain specific approaches and solutions. Hence, cyber security in smart grid considers specific communication protocols in various domains, such as the Distributed Network Protocol (DNP3.0), Modbus, IEEE Std. C37.118 and IEC 61850 [30]. Open research areas on cyber security for the smart grid includes confidentiality, integrity and availability, authentication, and vulnerability assessment.

\subsubsection{Network Communications}

In smart grid operations, power utilities and costumers use a variety of public and private communication networks, both wired and wireless. Utility network communication applications cover residential meters, transformer meters, feeder meters, and field distribution automation communication such as re-closers, switches, voltage regulators and capacitor banks. Prosumer network communication facilitates mainly in home area network (HAN) to intelligently manage devices. Wireless machine-to-machine (M2M) communication between smart meters eliminate human intervention necessity to operate the grid intelligently. Wireless communication is one of the key aspects of realizing the smart grid visions using different technologies such as IEEE 802.11 based wireless local 
area network (LAN), IEEE 802.16 based WiMAX, 3G/4G cellular, long term evolution (LTE), ZigBee based on IEEE 802.15, and IEEE 802.20 based MobileFi.

\subsection{Active Distribution Networks}

ADN is an emerging concept that refers to complicated operation of bidirectional energy flow as results of high penetrations of renewable DERs. ADN is the new form of power distribution networks blended with widely known microgrid concept [31]. A number of investigations about the technical and economic advantages of ADNs are presented in literature. This includes cost-benefit analysis on annual and investment reduction basis [32], [33], voltage control with DERs and protection application [34], expansion planning with distributed generation and load response uncertainties [35], and the dynamic optimal power flow for ADNs [36].

As increasing numbers of distribution feeders gain ADN capabilities with microgrid penetrations, the availability of providing auxiliary services with coordinated bidirectional power flow enhances. In order to implement a futuristic ADN, the interoperability with transmission network is imperative. The highly capable interaction can be only possible with advanced communication infrastructure along with wellestablished operation layers. The technical features of an ADN make it suitable for exploiting of storage reserves in dispersed microgrids and deferrable loads. From the utility point of view, ADN can be considered as controllable resource. The flexible control can be triggered through price signals to captivate private microgrid entities which aims to maximize profits and commercial electricity users who wants to reduce bills. However, in emergency conditions, to achieve a stable and secure operation, the dispatch and load 
shedding signals should be mandatory without violating non-deferrable loads. ADN can be considered sum of the distribution feeders which are connected to lower voltage level of step-down substation transformer. A number of distribution feeders are connected to main bus where DERs and loads dispersed along the feeder. Figure 1.2 shows a representation of future distribution network overview. Microgrids and deferrable loads are expected to be penetrated along the feeders. ADNs are closely coupled with the transmission network to enable coordinated wide-area closed loop control to increase resiliency. In case of emergency conditions, some parts of the distribution network would be partitioned until the complete network recovery is accomplished.

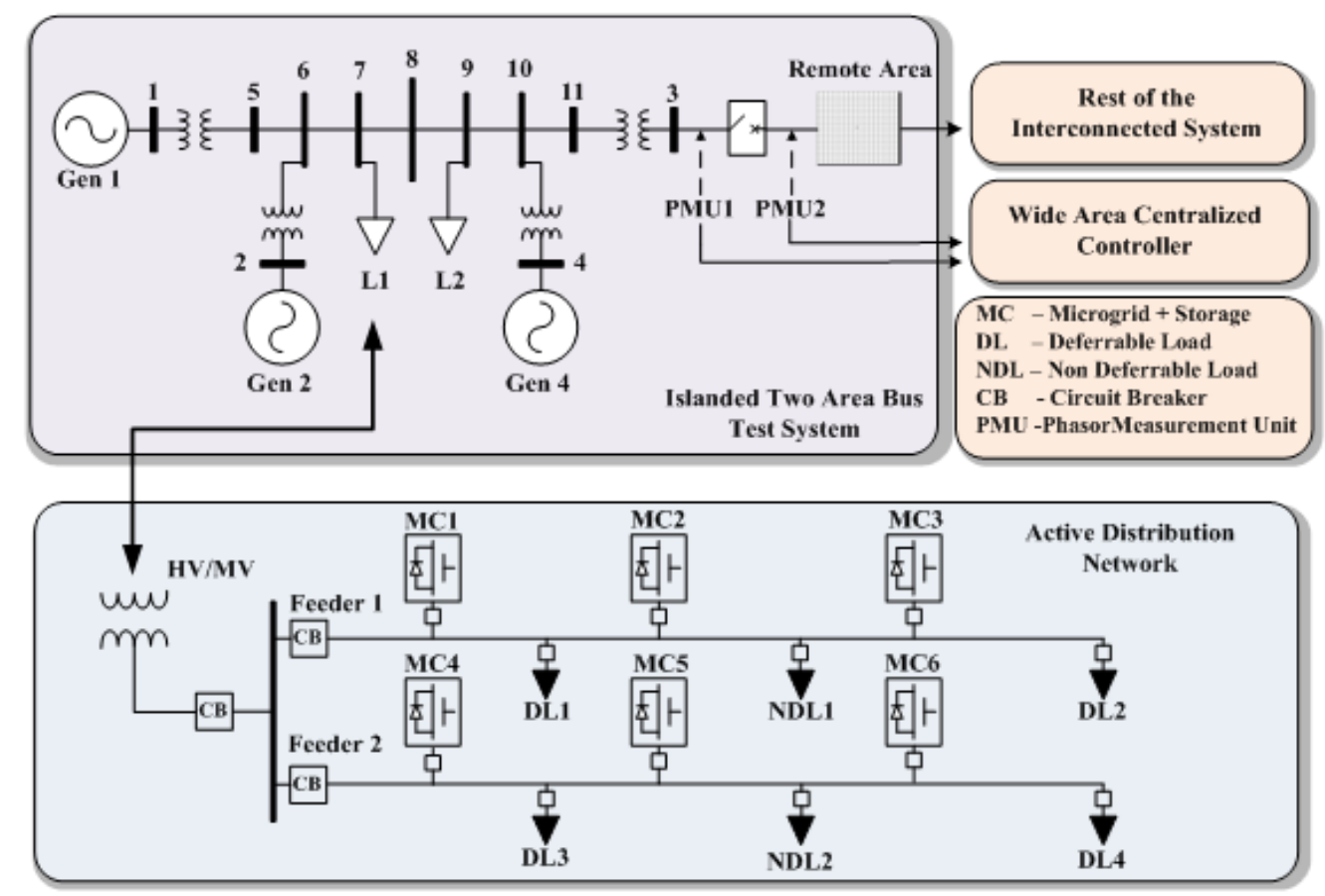

Figure 1.2: Active distribution network overview.

\subsubsection{Information and Communication Technology}

Optimization, automation and control of the smart grid are based on grid-integrated near-real-time communications between cyber-physical system sensors and actuators. Advanced communication technologies are essential to save energy, reduce costs and 
increase the reliability of the grid [37]. Cyber-physical infrastructure is the major driving force of the future smart grid vision as a foundational support system, meanwhile the key challenge for actual field deployment. Utilities and independent system operators seek proper ways to implement future smart grid concepts easily and securely for different application layers, such as metering, monitoring, operation, protection, automation and markets. Modern sensor networks stimulate the flexibility and application range for wide area implementation of smart grids. To conduct wireless communication experiments comparable to a real world environment, there is a significant need for wireless communication testbeds specifically for smart grids. This backbone would allow more dynamical topology changes and field deployments. Wired communication technologies for distributed control applications such as market negotiations, power sharing, aggregation, and protection may not be adequate.

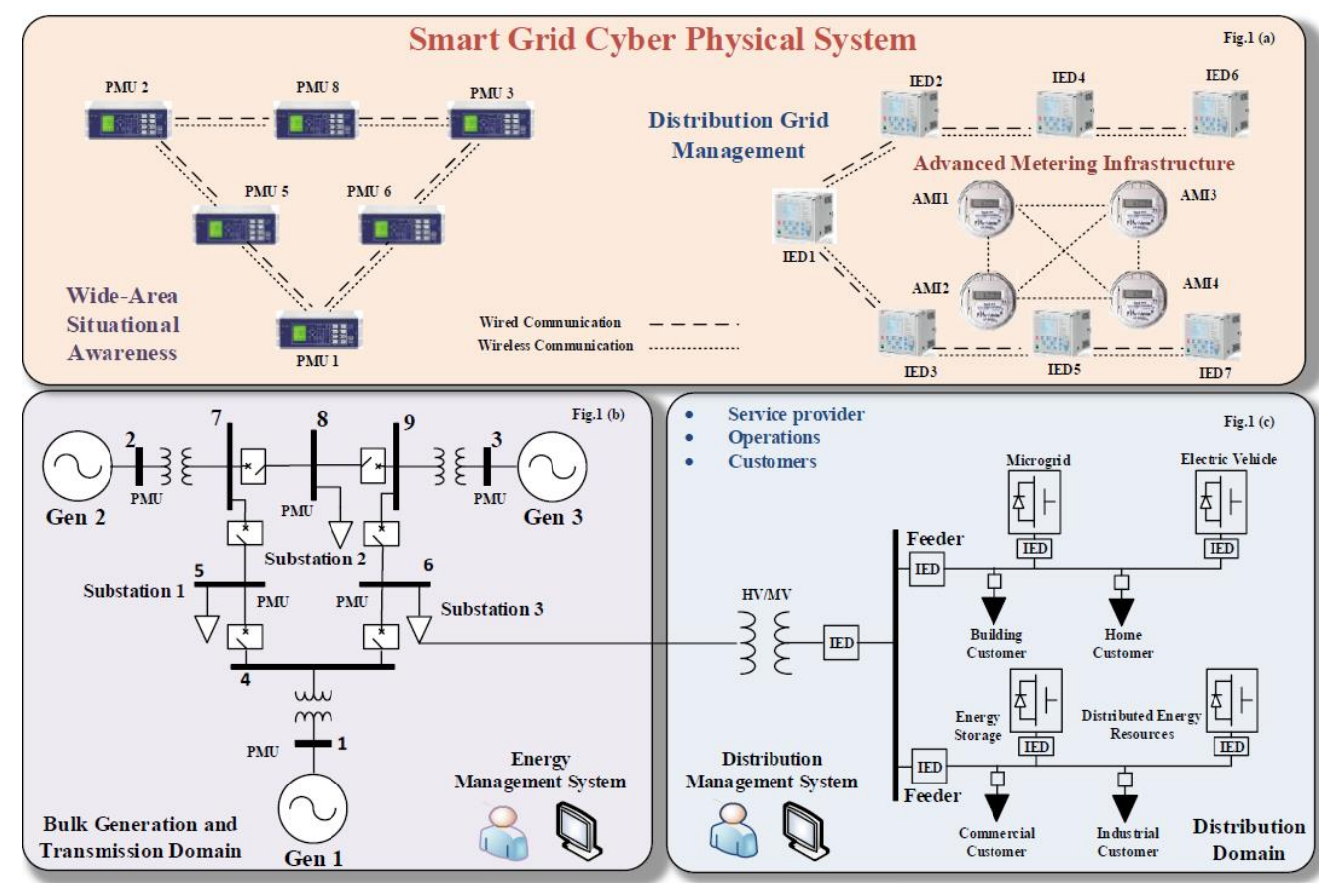

Figure 1.3: Smart Grid domains and research areas. 
Therefore, the smart grid requires an extensive wireless communication research effort including new protocols and enhancing the existing ones. Figure 1.3 shows the complete overview of smart grid domains and research areas.

\subsubsection{Security \& Privacy}

Any research domain in smart grids should be accompanied with a cyber-physical interface [38], [39]. Thus far, many security studies focused on intrusion detection [40] and attack demonstrations. However, to achieve the future goals and provide a robust mechanism, further countermeasure techniques and algorithms should be developed and verified in cyber-physical smart grid testbeds. Online and offline forensic analysis research should be encouraged. Encryption and certificate methods need to be studied.

\subsubsection{Communication Networks}

The recently emerged software defined networking (SDN) paradigm can perfectly address resilient and secure data collection challenges by splitting controls of networks and data flow operations [41]. The major goal of SDN is to interact with the switches, and thus create an open networking architecture for everyone [42]. In this way, one can get a global view of the entire network and make global changes without having to access to each device's unique hardware. Therefore, various large-scale network architectures can be deployed and maintained with ease while still featuring resiliency and robustness. The data

communication of smart grid necessitates upgrading the existing infrastructure with various components. SDN-enabled devices would offer a resilient communication infrastructure in all smart grid domains [43]. 


\subsubsection{Data Interoperability}

Interoperability is the ability of two or more devices to exchange information and work together in a system [44]. This is achieved using published objects and data definitions, standard commands and protocols. The smart grid interoperability requirements are clear [45]. All stakeholders need to use commonly agreed upon compatible data exchange formats for fully integrated framework. The power system framework must work together not just across the technical domains of the smart grid but across stakeholder communities in enterprises not part of the existing utility industry [46]. The future challenges include expanding existing protocols and providing ongoing definition of information models and information exchange requirements [47].

\subsubsection{Decentralized and Distributed Control}

Centralized control methods of operation require a high performance central processing unit and are more susceptible to single point failures, where managing the vast amount of data generated from the extensive deployment of smart devices becomes infeasible. In contrast to centralized control, the emerging smart grid concept is in a trend to adopt distributed methods as a result of the highly dynamic behavior of the power grid. Distributed control approaches intend to provide autonomy for different control layers by enabling an event-driven peer-to-peer communication structure, where central control schemes mainly rely on master-slave interactions [48]. In power system applications, the implementation of distributed control is established using multi-agent frameworks, which are composed of interacting multiple intelligent agents to achieve a global or a local objective function. 


\subsubsection{Multi-agent Systems}

In power system applications, the implementation of decentralized control is established using multi-agent frameworks, which are composed of interacting multiple intelligent agents to achieve a global or a local objective function. Multi-agent based schemes are widely applied to power system controls in literature; including self-healing, resilient grid automation [49], [50] and power system protection [51], [52]. Multiagentbased microgrid control draws considerably more attention than any other smart grid applications as one of the main assets of emerging AND concept [53]. Multi-agent based hierarchical hybrid control for microgrid is proposed to maintain voltage and maximize economic benefit [54]. Although many multiagent-based microgrid studies have been reported, none of the previous works address the industrial data interoperability, which is crucial for actual field deployment. Most of the works have been validated in simulation environments where data interoperability of the cyber-physical components have never been an issue. In reality, an agent requires to interact with its environment through sensors and actuators. A sensor acquires the data from the outside world and the actuator responds according to the agent's decision. For actual implementation of decentralized control schemes in power systems, it is imperative to link multi-agent objects to distributed industrial control systems such as smart meters, PMUs, IEDs and programmable logic controllers (PLC). The required interface is established through a combination of interoperable data and protocols.

\subsubsection{Microgrids}

The microgrids offer an essential solution to enable a resilient grid infrasturture since they have the ability to continue operating in case of a utility outage [55]. A microgrid 
can be viewed as a cluster of DERs, which have a connection to main utility grid. Microgrids could be composed of conventional or renewable DERs. Microgrids mostly depend on intermittent renewable resources [56]. This may introduce important stability problems especially during the islanded operation. Minor stability problems can cause cascaded outages which would result in a large scale blackout if proper action is not taken on time. An effective way to prevent such risks, ensure robustness, and resiliency is to operate the grid in smaller regions by spliting the network when necessary.

Grid-connected operation is the mode when the microgrid is coupled to the main utility grid in order to deliver/receive power to/from the grid. In the grid-connected mode, the frequency and voltage of microgrid can be supported by the stronger power system. Therefore, a complex regulation control is not necessary [57]. The transition process from grid-connected to the islanded operation mode should be smooth without violating power quality indicators, such as voltage and frequency levels. Microgrids should be able to provide the real and reactive power requirements of the loads in the islanded area while regulating the voltage and frequency within limits.

\subsection{Wide-Area Monitoring and Control}

Wide-area monitoring and control aims to provide protection for globally interconnected transmission networks. Measurements from dispersed substations are collected in a central controller to monitor system status in very precise synchronization. The recent demolishing catastrophic events have focused attention on the resilient and reliable electric grid which requires a proper risk assessment and emergency preparedness. Essentially, power system is able to withstand disturbances and contingencies depending 
on the design and operating conditions. However, an unusual combination of circumstances and events may cause a portion of the interconnected system to separate completely and form one or more electrical islands [58]. Although the separation process is expected to be controlled by enabling minimum load-generation unbalance, most of the cascading power system collapses result uncontrolled islands where the system recovery may not be assured [59]. In a wide-area protection and control scheme, central control units may force local substations to carry out mandatory emergency and remedial actions such as controlled islanding in case of blackout. Under-frequency load shedding schemes and aggregated distributed generation control can be adopted according to global monitoring feedback.

\subsubsection{Phasor Measurement Units}

Synchronized phasor measurements offer an opportunity for measuring power power system frequency which eliminates wrong frequency detection [60]. The frequency measurement is primarily obtained from rotor speed of connected generator and the phasors give the fundamental frequency components of the voltages therefore harmonics can have an effect on frequency on independent grids, energy transfer between neighbour networks. New generation PMUs offer advanced solutions by means of communication and protection with network communication protocols such as IEC 61850 and IEEE C37.118. IEC 61850 is a widely accepted substation automation protocol that can be fully implemented on a global approach on communication and information handling [61], [62]. It also fulfill the needs of the time critical communication tasks by proper GOOSE (Generic Object Oriented Substation Event) messaging for horizontal communication. GOOSE automatically broadcasts messages containing status control and measured values on network for use by other intelligent electronic devices. This communication ability enables 
solution for complex power system control and decision making process. The C37.118 standard defines synchronized phasor and frequency measurements in substations along with methods and requirements for measurement verification [63], [64]. Moreover communication and recording of phasor measurements are covered in C37.118 standard. IED based relays are high speed line protection relay feasturing single-pole and three-pole tripping and reclosing with synchronism check, circuit breaker monitoring, circuit breaker failure protection and series-compensated line protection logic. PMUs can provide information to power system operator with synchronized measurements for positive sequence voltage and current, phase angles, local frequency, rate of change of frequency (ROCOF), harmonics, negative and zero sequence quantities along with the time stamped information from globa measurement based upon phasor estimation.

\subsubsection{Time Synchronization in Power Systems}

The time synchronization is generally established using IRIG-B code by a satellite clock to have a proper time reference value from a GPS clock to accomplish a reliable synchronized measurements from the whole network [65]. A PMU should be capable of receiving reliable and accurate time over a broad geographic zones. GPS can provide the coordinated universal time (UTC) with a sufficient accuracy to keep the total vector error (TVE), frequency error (FE) and ROCOF within the acceptable limits. In a $60 \mathrm{~Hz}$ system, 1 micro seconds represents 0.022 degrees. $1 \%$ TVE corresponds to 26 micro second error [66]. It is recommended to have a time reference at least 10 times more accurate than this value. The latency is an important factor while evaluating the performance of PMU and phasor data concentrator (PDC). The latency is assumed to be the maximum time interval between the time stamped data and when the data becomes available at PMU output 
expressing the first bit of data. The latency is dependent on various factors, such as sampling window, calculation/estimation method, PMU processing time and reporting interval.

\subsection{Problem Statement}

As highlighted in previous sections, decentralized control is of a significant importance in future smart grid operations in both wide area control and distribution networks. In power systems, decentralized control is accomplished by advanced multiagent frameworks. Multiagent systems have been well-studied in computer science for various applications. However, the real-time application of multiagent systems in power system operations come with several challenges. Interoperability is one of the major challenges to accomplish a complete smart grid infrastructure due to the large amount of processed data from different vendor and communication protocols. IEC 61850 is the new international standard of communications, which enables the integration of all substation functions in self, such as protection, control, measurement and monitoring. However, the smart grid concept covers an extensive control, automation and protection applications such that a single standard may not meet all the required forms of monitoring and information exchange demands. Considering the emerging ADNs, new energy market policies are necessary such as implementation of real-time auction models and scheduling the dispatch of DERs. Hierarchical control of microgrids require interaction with utilities for dynamic adjustment of the primary, secondary and tertiary control levels. Taking into account the mentioned requirements of the future grid, this dissertation proposes utilization 
of advanced intelligent multi agent frameworks with a flexible ability to create tailor-made decentralized control schemes while following the legacy protocols.

In terms of power system protection, the conventional protection schemes require radial protection devices connected in series, and the trip characteristics of the downstream protective device must be faster that the upstream devices. However, the integration of multiple distributed generators (DGs) would form extremely complicated meshed microgrid networks with dynamically connecting/disconnecting DGs. This situation results in volatile network impedances, and accordingly stochastic fault currents. Thus, new challenges emerge by relying on predefined fault current settings on IEDs. Traditionally, conventional protection is operated for high current levels in distribution networks. However, in islanded microgrid operation, the high fault currents from the utility are not present. Various fault levels would prevail due to the wide range of short circuit impedances, and it is likely that multiple IEDs reporting fault locations for the same fault, yet actual clearing requirements are different. The emerging challenges compel microgrids to adapt communication-based cooperative methods. Distributed control architectures inherently rely on tight coupling between the cyber and physical layers, thus the networked information systems are the core components of microgrid interaction. In a dynamic network, the implementation of a centralized control with access to all IEDs in the system is not realistic. Major drawbacks of the centralized methods are the requirement of high communication capability with a powerful central controller, and being susceptible to single point failure. This can easily jeopardize the system with a complete collapse. Due to their inherent resilience, agent-based distributed adaptive approaches can be used to self- 
check and react accordingly to prevailing environment conditions even with communication constraints.

As intermittent generation profiles introduce more stress due to the sudden imbalance in supply and demand, power systems are becoming more dependent on online aggregated support of networked utility-independent private microgrids. During the aggregation process, microgrid operators' preferences and bidding behaviors are priority. New aggregation approaches are required considering the individual behaviors of the networked and geographically dispersed independent microgrids. Agent-based systems consistently provide higher profits than their human counterparts, yet these agents still might require occasional human operator support. Thus far, agent based market and auction models have been implemented to maximize the benefits of generation units by analyzing the effects and evaluation of bidding strategies. However, intentions and behaviors of the agents should be considered, and self-decision strategies should be modeled to maximize the generated revenue on behalf of microgrid operators. As selfish and greedy agents' preferences are priority as independent microgrid bidders, behavioral modeling of the independent agents is an open research problem.

\subsection{Research Objective}

The decentralized multiagent system is an effective solution to provide resiliency and interoperability with reliability in various power system applications, such as those in hierarchical control, protection, aggregator, economic dispatch, as well as cyber-physical system security. A comprehensive, proper and well-designed multiagent system framework can play a significant role in improvements of the power system efficiency, 
resiliency and reliability. This dissertation involves the development and experimental validation of various agent-based decentralized and distributed control techniques for wide area and active distribution network control of power systems. Furthermore, its real-time application and prototype implementations verify the performance of the system.

The main research objectives and contributions of this dissertation can be summarized as follows:

- To accurately design, model and implement a hybrid AC/DC microgrid system incorporating industrial data acquisition, control and protection technologies and enhanced control capabilities at the Energy Systems Research Laboratory, Florida International University. The industrial microgrid system is a hardware/software-based setup with synchronous generator, inverter-based DER, and energy storage capability. This platform is equipped with a state-of-the-art monitoring, control and protection capabilities using PMUs, IEDs, PDC, PLC and a real-time automation controller (RTAC). The developed system includes implementations of advanced control strategies including seamless islanding, seal-healing capability, adaptive protection, and synchrophasor assisted automation.

- To design, develop and implement a hybrid multiagent framework for microgrid and multi-microgrid operations featuring legacy information and communication protocols at the Energy Systems Research Laboratory, Florida International University. The multiagent framework implements IEC61850, open connectivity unified architecture (OPC UA) and foundation of intelligent physical agents (FIPA) protocols as information 
interoperability. Cloud communication is used to provide solutions to embedded systems in the internet of things (IoT) which significantly reduced the operational costs compared to dedicated high bandwidth side area links. IEEE 802.11s-based mesh network using hybrid wireless mesh protocol (HWMP) is used for internal local area network communication.

- To designed and experimentally evaluate a bi-directional distributed optimization framework featuring local and interconnected microgrids. The developed optimization scheme should consider individual microgrids implementing a fully distributed optimization algorithm, and a quasidecentralized optimization approach using cloud communication on a global framework featuring interconnected microgrids. The underlying communication architecture also should consists of bi-level communication network consisting of peer-to-peer wireless mesh networks and cloud communication infrastructure, using legacy protocols particularly such as IEC 61850 , to achieve certain delay performance so that optimization convergence is guaranteed.

- To develop and experimentally evaluate an advanced protection scheme to overcome selectivity challenges of low inertia inverter and synchronous generator-based harmonized microgrids. The developed protection scheme should be able to properly coordinate presence of power converters and relatively tolerate low inertia. The developed scheme should be practical and address both overcurrent and frequency selectivity. Furthermore, the 
selectivity mechanism should consider the RAS of the microgrid after a fault instant based on feeder characteristics and the location of the relays.

- To develop and experimentally evaluate a multi-agent based game theoretic reverse auction model for microgrid market operations featuring conventional and DER. The experiments should be conducted on a grid connected microgrid consisting of a synchronous generator, two inverterbased generation units and a combined load. The multi-agent based platform needs to be applied to monitor, control and perform the reverse auction process of DERs. A competitive game-theory reverse auction model should be investigated to schedule the DER unit commitment with an hourahead market approach for the twenty-four hours of the day.

- To develop and experimentally evaluate auction framework for aggregation of networked utility-independent private microgrids. During the aggregation process, microgrid operators' preferences and bidding behaviors should be taken into account as priority. The bidding behavioral modeling and auction architecture should consist of a central aggregator and networked microgrid agents. The bidding behavioral states should be mathematically formulized to determine belief-updates and short-term policy determination in order to maximize the individual benefit of each agents. 


\subsection{Original Contributions of the Dissertation}

In order to verify the contributions of this dissertation, a laboratory-scale hardware/software-based hybrid microgrid and as the cyber-physical infrastructure multiagent framework is developed. This microgrid research platform is equipped with synchronous generator, inverter-based DER, energy storage and features industrial data acquisition, control and protection technologies. The enhanced control capabilities include seamless islanding, seal-healing capability, adaptive protection, and synchrophasor assisted automation.

The cyber-physical multi-agent framework implements FIPA, OPC UA, and IEC 61850 standards. The IEC 61850 logical node concept is extended using FIPA based agent communication language (ACL) with application specific attributes and deliberative behavior modeling capability. The OPC UA middleware is adopted to enable a real-time publisher-subscriber interoperability mechanism between platforms. The proposed multiagent framework is validated in a laboratory based testbed involving developed IED prototypes and actual microgrid setups. Experimental results are demonstrated for both decentralized and distributed control approaches. Secondary and tertiary control levels of a microgrid are demonstrated for decentralized hierarchical control case study. A consensus-based economic dispatch case study is demonstrated as a distributed control example. It has been shown that the developed agent platform is industrially applicable for actual smart grid field deployment.

In this dissertation, two agent-based protection schemes are developed: autonomous microgrid protection using distributed protection and fully distributed approach for meshed type of networks. Protection of an autonomous microgrid requires 
special considerations compared to large scale distribution networks due to the presence of power converters and relatively low inertia. In this dissertation, we introduce a practical overcurrent and a frequency selectivity method to overcome conventional limitations. The proposed overcurrent scheme defines a selectivity mechanism considering the RAS of the microgrid after a fault instant based on feeder characteristics and the location of the IEDs. A synchrophasor-based online frequency selectivity approach is proposed to avoid pulse loading effects in low inertia microgrids. Experimental results are presented for verification of the proposed schemes using a laboratory based microgrid. The setup is composed of actual generation units and IEDs using IEC 61850 protocol. The experimental results are in excellent agreement with the proposed protection scheme.

This dissertation also includes an agent-based distributed approach for meshed microgrid protection with a fully distributed approach where each agent can only communicate with neighbor agents. A consensus-based auction algorithm is proposed for cooperative fault localization method in islanded mesh microgrids. A distributed depthfirst search (DDFS) algorithm is introduced for adaptive agent discovery and network matrix reconfiguration after fault clearance. Experimental and simulation results are presented for verification of the proposed algorithms. Results show that the proposed distributed algorithms offer promising communication-assisted solutions to overcome microgrid protection challenges.

As intermittent generation profiles introduce more stress due to the sudden imbalance in supply and demand, power systems are becoming more dependent on online aggregated support of networked utility-independent private microgrids. During the aggregation process, microgrid operators' preferences and bidding behaviors are priority. 
New aggregation approaches are required considering the individual behaviors of the networked and geographically dispersed independent microgrids. In this dissertation, a novel bidding behavior modeling and an auction architecture consisting of a central aggregator and networked microgrid agents is developed. The bidding behavioral states of the microgrid agents are formalized as POMDP for the belief updates and short-term policy determination in order to maximize individual profit. A reverse auction model is adopted to enable competitive negotiations between the central aggregator and networked microgrid agents. The auction and aggregation processes are implemented in power system control area along with an AGC scheme to contribute frequency control. The proposed AGC and auction mechanism are verified with an industrial multi-agent framework in a laboratory-based real-time application.

\subsection{Dissertation Outline}

This dissertation is organized in eleven chapters, including the current chapter, which contains a brief introduction to the dissertation and statement of the problem. Chapter 2 is presents a description of the hybrid industrial hybrid AC/DC microgrid setup developed as one of the verification tool for this dissertation. The components of this

system that include synchronous generator, power electronic converter, photovoltaic emulator, energy storage, load emulators. Also, industrial automation, protection, and control infrastructure featuring PMUs, IEDs, and PLCs is included in this chapter.

Chapter 3 investigates the operation and performance issues related to hybrid AC/DC microgrids. The focus of this study is to achieve effective resiliency in a hybrid microgrid consisting of different DERs such as synchronous generators and inverter-based. 
The main objective of this chapter is to present a proper voltage and frequency control during cascading islanding by switching appropriate operation modes according to the DER type. Various simulation and experimental tests forming different islanding conditions are performed and the results are analyzed.

Chapter 4 explains the development of synchrophasor assisted microgrid operation with a time-synchronized and high-sampling rate approach. High sampling rate data acquisition and monitoring and the developed supervisory control and data acquisition (SCADA) interface presented. Synchrophasor-based islanding detection techniques and how synchrophasors improved resynchronization process is demonstrated. Protection and event analysis are discussed for postmortem analysis. Experimental tests of the ancillary service, hierarchical frequency and voltage control, performance analysis under pulsed loads are presented.

Chapter 5 proposes a new protection scheme for autonomous microgrids using agent based distributed communication. The agent based distributed communication architecture is used to overcome conventional overcurrent and frequency protection selectivity challenges. A new selectivity mechanism is proposed based on feeder characteristics and the location of the IEDs considering the RAS of the microgrid after a fault instant. An online frequency selectivity approach is proposed to avoid pulse load effects in low inertia autonomous microgrids. IEC61850 framework and GOOSE protocol messaging are used to enable high-speed peer-to-peer communication capability. To demonstrate the proposed protection scheme, real-time laboratory experiments are performed using the developed industrial hybrid AC/DC microgrid. 
Chapter 6 focuses on a fully distributed approach for protection of meshed microgrids. The proposed scheme is based on a distributed approach for microgrid protection with communication constraints, where each agent can only communicate with its neighbor agents. The application of consensus-based auction algorithm for fault localization, and the clearing processes are discussed. A DDFS algorithm is introduced for adaptive dynamic agent discovery and network adjacency matrix reconfiguration after fault clearance. Simulation and experimental results are presented.

Chapter 7 presents a novel multiagent interoperability framework. This framework provides a flexible multi-agent framework for decentralized hierarchical and distributed control of microgrids merging the IEC 61850, FIPA and data OPC UA standards. The OPC UA middleware is adopted to provide a real-time publisher-subscriber interoperability mechanism between platforms. Prototype IEDs are built integrating the protocols, and deployed in actual microgrid hardware setups. Case studies are demonstrated for both decentralized and distributed control approaches. Secondary and tertiary hierarchical control levels of a microgrid are demonstrated for decentralized control case study. A consensus-based economic dispatch case study is demonstrated for distributed control of the microgrid.

Chapter 8 presents implementation of a remote access smart grid test bed using cloud communication technology. The cyber-physical infrastructure of the laboratory test bed at Florida International University Smart Grid test bed is introduced. A substation model is used to demonstrate the remote access platform along with the industrial generators, PMUs, IEDs and PLCs. Industrial data sets, models and metrics are addressed. A realistic protection scheme is demonstrated as well as laboratory based information 
models. It has been presented that cloud communication can be successfully implemented for actual smart grid power systems test beds.

Chapter 9 presents multiagent-based fully distributed microgrid control and communication network design to support distributed control/optimization in smart grids. In this chapter, we investigate distributed optimization using a new fully distributed optimization method using diffusion strategy with a hierarchical approach to maintain frequency regulation and economic dispatch. The proposed control scheme employs peerto-peer communication among the agents replacing the need for a centralized controller to maintain system frequency regulation and additionally performs optimal economic dispatch simultaneously. The results are compared with centralized and consensus-based optimization algorithms.

Chapter 10 presents a grid-connected real-time operation of a multi-agent based microgrid system for market operations of renewable and conventional resources in a microgrid incorporating PMUs and IEDs. A multi-agent based platform is utilized to monitor and control microgrid operations through sensors and actuators of generation units such as the governor and inverter control of the power storage device. A multi-agent based game theory reverse auction model is utilized to enable a competition between DERs in order to obtain the cheapest power supply for a lumped load on a microgrid. The realistic study is successfully implemented and operated using developed multiagent framework.

Chapter 11 provides a novel aggregation framework for networked auctioning agents for a provision of local area frequency support in case of a sudden supply/demand imbalance. A reverse auction mechanism for AGC scheme to enable a competition among microgrid agents for provision of the local area frequency support by a central aggregator 
controller. The POMDP technique is used to determine a short-term bidding policy of the microgrid agents by online belief updates. To demonstrate effectiveness and communication architecture of the proposed auction architecture, a real-time laboratory experiment is performed.

Chapter 12 provides general outcomes of the results and the conclusion of this dissertation. Also, some suggestions and recommendation for further work related to the topics covered. 


\section{DESIGN AND IMPLEMENTATION OF INDUSTRIAL MICROGRID}

\subsection{Introduction}

In this chapter, the design and implementation of an industrial hybrid AC/DC microgrid is presented. This microgrid infrastructure is used for experimental verification of the developed algorithms in the sections to follow. The developed test setup was implemented at the Energy Systems Research Laboratory at Florida International University. The hardware of the hybrid microgrid includes a synchronous generator, an inverter-based distributed energy resource, energy storage, and load models. The automation, protection, and control part of the microgrid contains PMU, PDC, RTAC, satellite clock GPS, IED, PLC, and advanced metering infrastructure. In the following section, the microgrid hardware components are described. Section 2.2 presents the generation units, storage, and load models. Microgrid automation, protection, and control devices are included in section 2.3.

Hybrid power systems are gaining popularity due to increasing microgrid deployments, which feature renewable power systems connected to low voltage AC distribution systems. Furthermore, the development of new semiconductor technologies and sustainable DC power sources, such as solar energy, are causing a resurgence in the use of DC grids. There has also been an increase in the use of DC loads, such as PEVs and light emitting diodes (LEDs), connected to the grid to save energy and decrease greenhouse

gas emissions. Power converters have presented new horizons for effective integration of $\mathrm{AC}$ and DC distribution networks in the context of microgrid operation. At the same time, 
various utility grids, and some hybrid microgrids, are increasing the penetration of renewable energy resources.

The development of a resilient and intelligent hybrid microgrid setup requires extensive deployment of a cyber-physical architecture. Apart from the communication infrastructure, the information domain has vital importance to facilitate an enhanced protection and control scheme. PMUs allow power system operators to measure timesynchronized phasor measurements, frequency, and rate of change of frequency (ROCOF) from the wide-area power system. The standard IEEE Std. C37.118 involves the use of synchrophasor measurements from power systems and the data information model.

Hybrid power systems face far more challenges when operating in islanded mode than they do in grid-connected mode. While in islanded mode, the AC side can no longer be viewed as an infinite bus, which results in load variations adversely affecting the frequency and voltage of the system. If the system has a high penetration of renewable power, the situation can be even worse. At any time, reactive and active power flow should be balanced between the AC and DC sides to maintain stability on both sides of the grid.

The developed microgrid architecture allows a comprehensive frequency and voltage control scheme for a hybrid AC/DC operation consisting of a synchronous generator, solar generation emulator, and bidirectional (AC/DC and DC/DC) converters. A bidirectional controlled AC/DC converter, with an active and reactive power decoupling technique, is used to link the $\mathrm{AC}$ bus with the $\mathrm{DC}$ bus while regulating the system voltage and frequency. A DC/DC boost converter, with a maximum power point tracking (MPPT) function, is implemented to maximize the intermittent energy generation from solar generators. Current controlled, bidirectional DC/DC converters are applied to connect each 
lithium-ion battery bank to the DC bus. Lithium-ion battery banks act as energy storage devices that serve to increase the system resiliency by absorbing, or injecting, power. Section 2.2 describes the hardware components of the microgrid. The automation, protection and control components are then presented in section 2.3.

\subsection{Microgrid Hardware}

The complete overview of the microgrid setup is illustrated in Figure 2.1. The hybrid microgrid involves a synchronous generation unit and an inverter-based DER. In addition to the local loads, which are connected to the point of common coupling (PCC) of the generation unit, the system has a global load on the main bus.

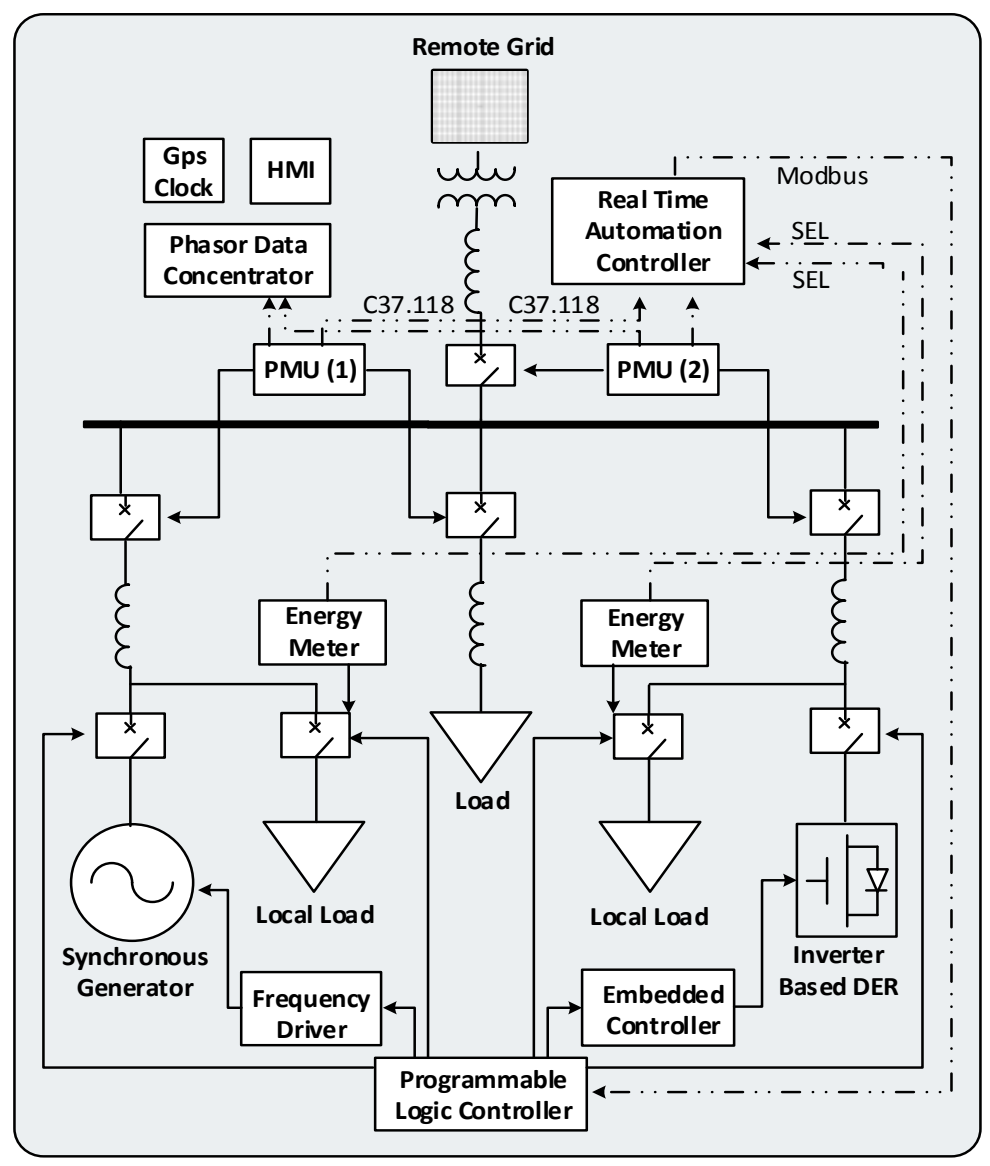

Figure 2.1: Microgrid architecture. 
The microgrid has a connection to utility grid, which enables both in grid-connected and islanded operation. The operational voltage level of the microgrid is $208 \mathrm{~V}$ rms lineto-line, which is the utility voltage level. PMUs are located on both sides of the PCC to utility grid connection. The internal microgrid consists of local loads, a synchronous generator, and inverter DER.

\subsubsection{Synchronous Generator}

A 13.8-kVA, 60-Hz, 208-V, and $1800 \mathrm{Rpm}$ AC synchronous generator represents a conventional synchronous generation unit. The machine is equipped with a half-wave phase-controlled thyristor-type automatic voltage regulator. Excitation current is derived directly from the generator terminals. An induction motor driven by a frequency driver is used to emulate the generator governor model. The frequency drive follows "vector speed control" that serves to keep the frequency constant. In order to emulate the PV buses, the prime mover of the generator is operated by a torque-controlled frequency drive, which regulates the output active power of the generation station. The torque, or speed reference, of the frequency driver is controlled by a PLC. The specifications of the synchronous generator are summarized in Table 2.1. Figure 2.2 shows the synchronous generator set including frequency driver and automatic voltage regulator.

Table 2.1: Synchronous generator parameters.

\begin{tabular}{|c|c|c|}
\hline Component & Parameter & Specification \\
\hline \multirow{3}{*}{ Synchronous } & $\mathrm{SN}_{\mathrm{N}}$ & $3 \mathrm{kVA}$ \\
\cline { 2 - 3 } Generator & $\mathrm{VN}_{\mathrm{N}}$ & $208 \mathrm{~V}$ \\
\cline { 2 - 3 } & $\mathrm{FN}$ & $60 \mathrm{~Hz}$ \\
\cline { 2 - 3 } & $\mathrm{XL}, \mathrm{Rs}$ & $6.87 \mathrm{mH}, 1.45 \Omega$ \\
\hline
\end{tabular}




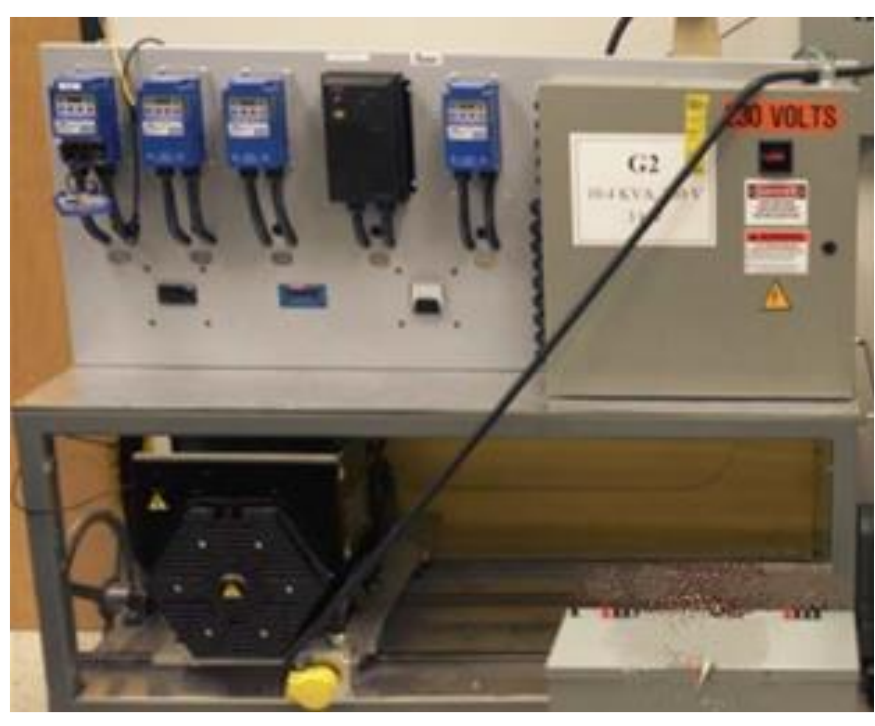

(a)

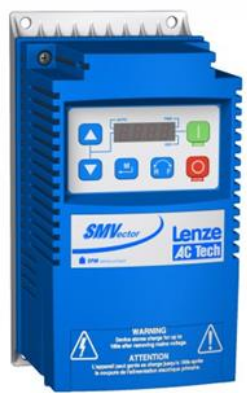

(b)

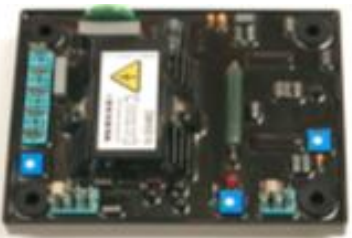

(c)

Figure 2.2: Synchronous generator set: (a) overview; (b) frequency driver; (c)

\subsubsection{Distribution Lines and Load Models}

The transmission lines are modeled as medium length $\pi$-type lines, which are composed of series inductors and two parallel capacitors. The load module is composed of 10 levels of parallel resistive loads, from $0-3 \mathrm{~kW}$ in steps of $300 \mathrm{~W}$, at nominal voltage. $[2 \times 72 \Omega+4 \times 144 \Omega]$ resistance models, which can be controlled by a PLC, are utilized. The inductive loads have been designed to provide reactive power consumption in the microgrid. The design is based on constant inductance, so they are similar to constant impedance loads in the case that the system frequency is constant. Otherwise, the frequency drift changes the inductance, and this change needs to be taken into account for further analysis. For emulating the circuit breaker, 530 V, 25 Ampere solid state relays are used. The trip/close signals can be controlled by 3-32 V DC supplied by the PLC. Figure 2.3 shows the transmission line resistive and inductive loads models. 


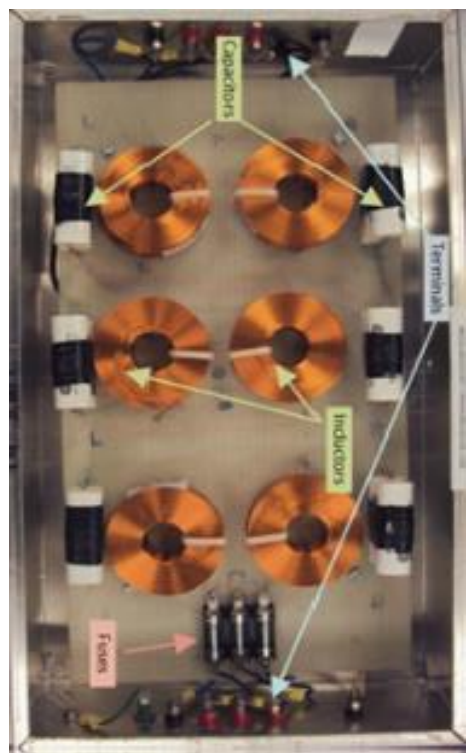

(a)

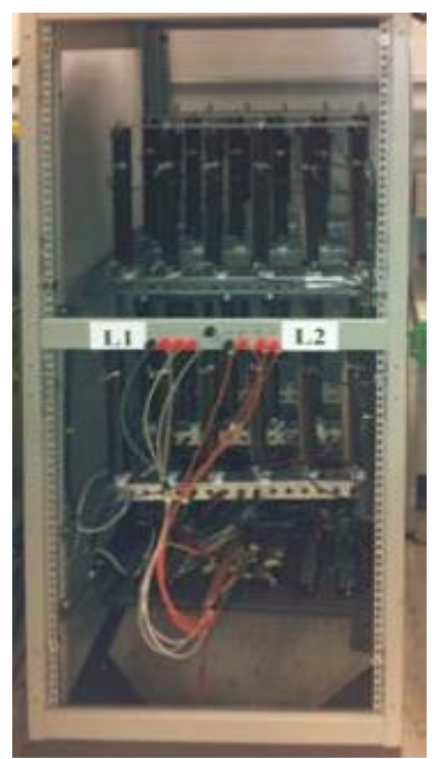

(b)

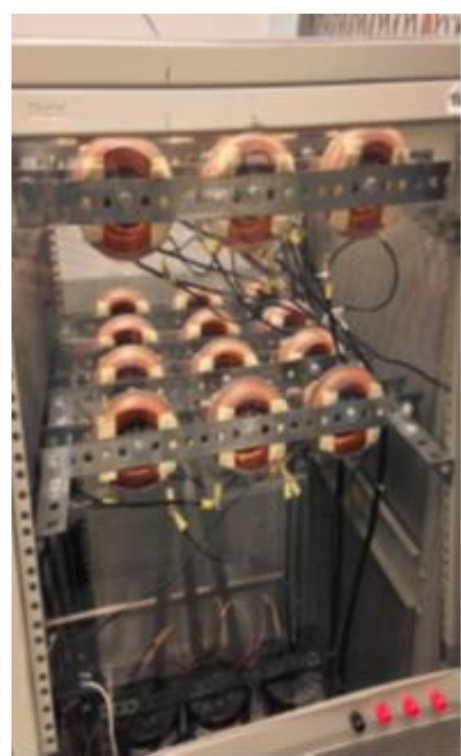

(c)

Figure 2.3: Line and load models: (a) medium length line; (b) resistive load; (c) inductive load.

\subsubsection{PV Emulator and Embedded Controller}

The PV panel can be viewed as a current source in parallel with a diode. The SunPower SPR-305-WHT solar cell, with 305W maximum output power, is used. Thirtythree cells are used in the configuration of 11 parallel strings, with 3 serially connected cells per string. Figure 2.4 shows the non-linear P-V and I-V electric characteristics of a single SunPower SPR-305- WHT solar cell. Under different levels of solar irradiations, the maximum power points of the power-voltage curves are associated with different output voltages. Also, under certain levels of solar irradiance, the output of the PV panel varies with different terminal voltages. The digital signal processor (DSP) based embedded dSpace control platform is used to control the inverter-based DER model. The internal active/reactive power generation of an insulated-gate bipolar transistor (IGBT) based inverter is programmed using the Matlab/Simulink platform. 

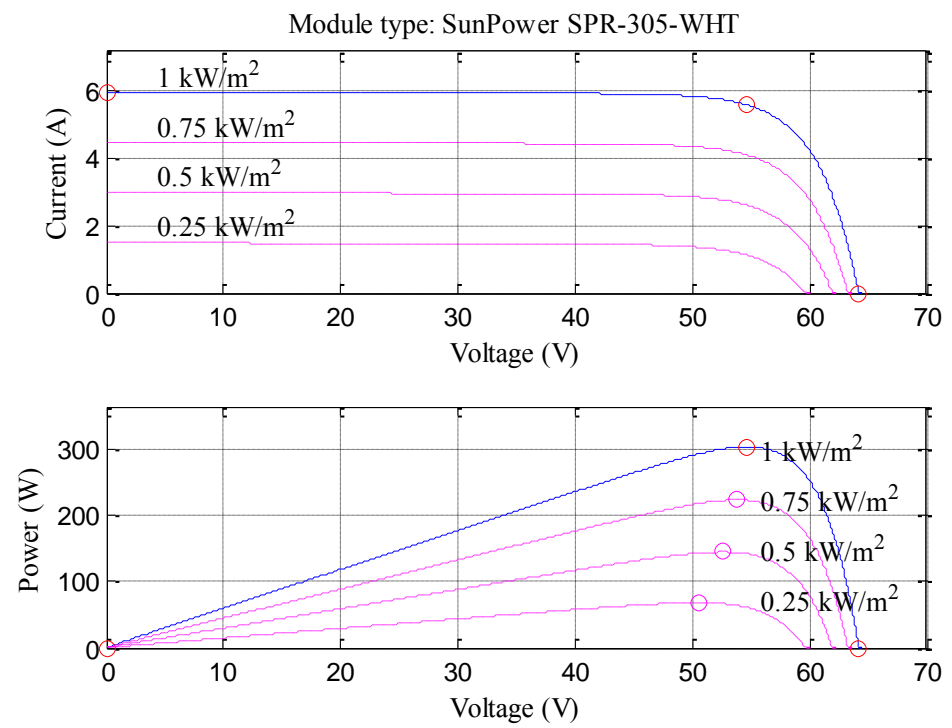

Figure 2.4: P-V and I-V electric characteristics.

The communication of this field actuator and PLC control is established through a built-in RS232 serial port. Figure 2.5 shows the PV emulator and embedded dSpace control platform.

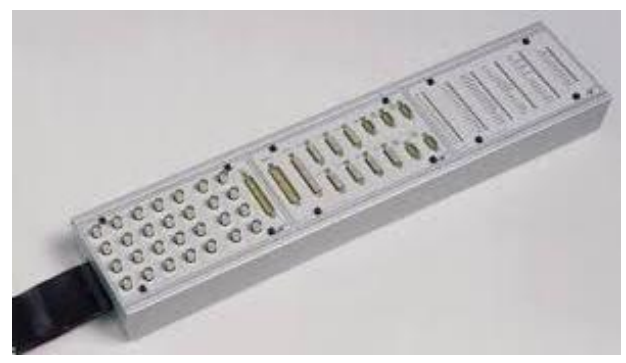

(a)

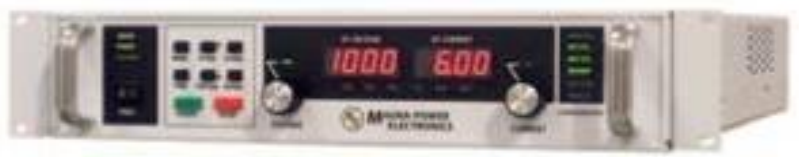

(b)

Figure 2.5: (a) dSpace embedded controller; (b) PV emulator.

\subsubsection{Bi-Directional $\mathrm{AC} / \mathrm{DC}$ and $\mathrm{DC} / \mathrm{DC}$ Converters}

The PV emulator is connected to the DC bus as a DC energy source through a DC/DC boost converter with MPPT functionality. Five 50Ah, lithium-ion battery banks, with $51.8 \mathrm{~V}$ terminal voltage, are connected to the DC bus through five bi-directional 
DC/DC boost converters. The rated voltages of DC and AC sides are $300 \mathrm{~V}$ and $208 \mathrm{~V}$ phase-to-phase, respectively.

\subsubsection{DC/DC Boost Converter with MPPT}

To maximize the utilization of renewable energy from the PV module, the boost converter should be operated in on-MPPT mode when the hybrid microgrid is connected to the utility grid. The battery banks in the microgrid can be used as an energy buffer, and the charging/discharging rates can be controlled based on the status of the output power from the PV farm and the power flow in the AC side.

\subsubsection{Bi-Directional DC/DC Converter}

The bi-directional DC/DC converters are used to connect the battery banks to the DC bus. The hardware setup consists of a battery bank, and a bi-directional DC/DC converter including the related measurement circuits. In grid-connected mode, these converters only regulate the battery banks' charging rates. Based on the SOCs of the battery banks and the power flow conditions in the $\mathrm{AC}$ side, the charging/discharging current references are generated to regulate the current flow in the converters. Each battery has its own bi-directional DC/DC converter, which means they can have different charging rates. The battery banks can inject power to, or absorb power from, the DC bus. Also, they can transfer energy between different battery banks if necessary.

\subsubsection{Bi-Directional AC/DC Converter}

The bi-directional AC/DC converter can take control of the AC side frequency and voltage amplitude. The DC bus voltage is regulated by controlling the charging and discharging of the battery banks, which also means controlling the current flow through the bidirectional DC/DC converter. The DSP-based embedded dSpace control platform is 
used to control the inverter-based DER model. The internal active/reactive power generation of the IGBT-based inverter is programmed using the Matlab/Simulink platform. The communication of this field actuator and PLC control is established through a built-in RS232 serial port.

\subsubsection{Energy Storage}

Five 50Ah lithium-ion battery banks, with $51.8 \mathrm{~V}$ terminal voltage, are connected to the DC bus through five bi-directional DC/DC boost converters. Figure 2.6 shows the bidirectional AC/DC converter, bidirectional DC/DC converter, and energy storage components.

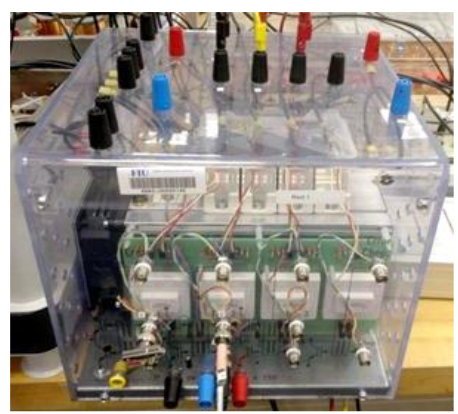

(a)

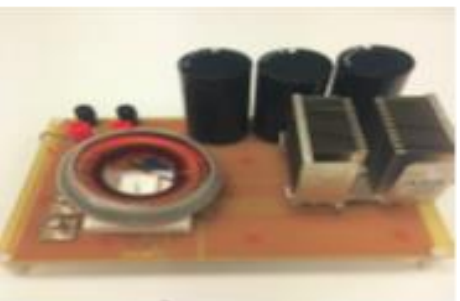

(b)

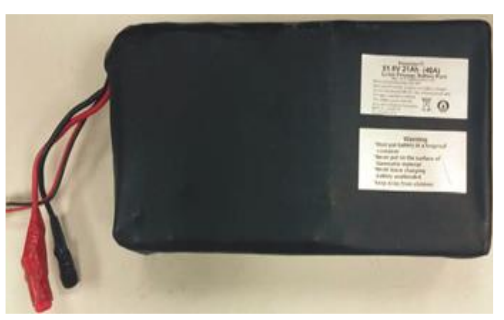

(c)

Figure 2.6: (a) AC/DC converter; (b) DC/DC converter; (c) battery bank.

\subsection{Microgrid Automation, Protection and Control}

A microgrid is the core structure of the complicated active distribution network concept, which enables multiple DERs to be run collectively by a central control entity. The communication of a microgrid, with the central control entity, is enabled through various network infrastructures. These networks include a wired network, such as high speed Ethernet and serial communications, and wireless networks with GPS synchronized 
time servers. Figure 2.7 illustrates the complete overview of the microgrid automation architecture including the components and the related communication protocols.

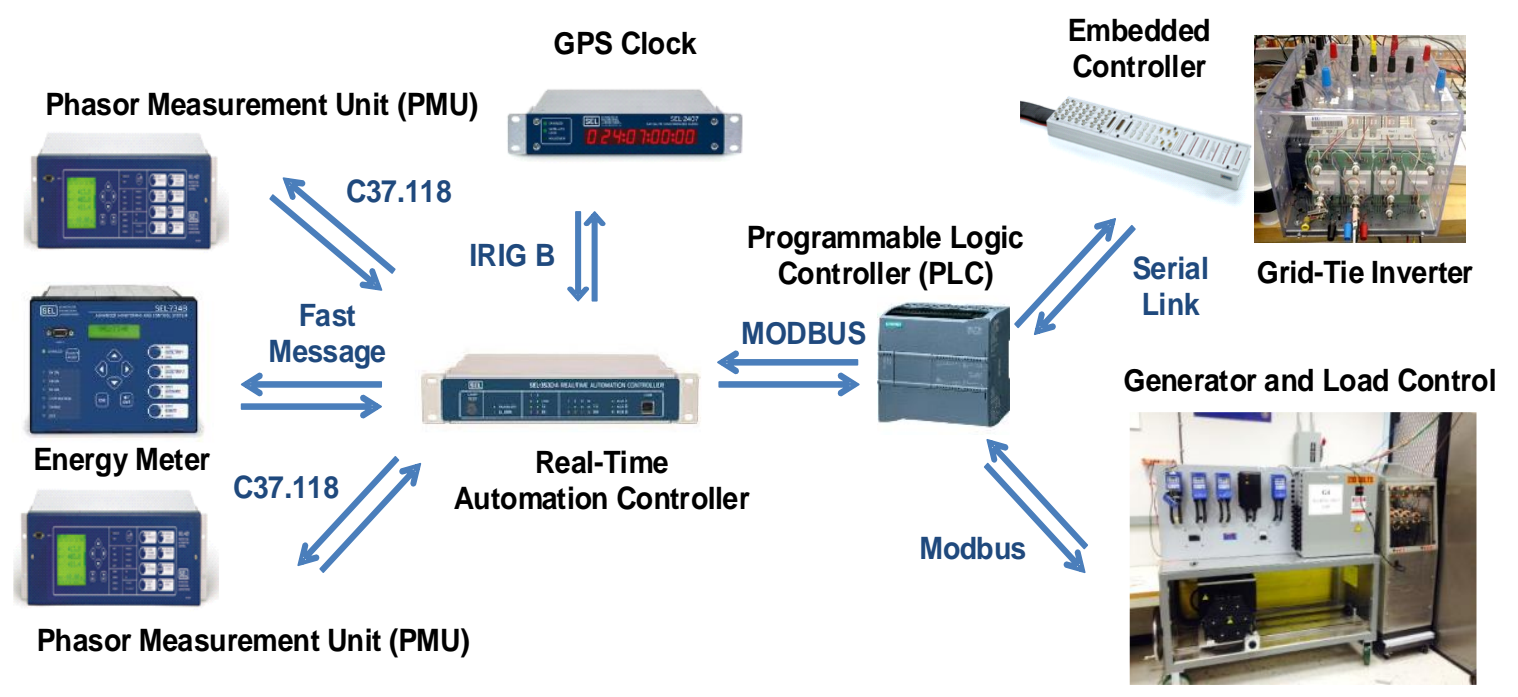

Figure 2.7: (a) Microgrid automation architecture

\subsubsection{Phasor Measurement Unit}

PMU applications explicitly deal with the system disturbances involved in the dynamic operation of complicated systems. High sampled data acquisition can reach up to 60 messages per second. In this setup, the reporting rate is 30 frames per second. The phasor representation of a sinusoidal AC signal is given as equation (2.1), where the phasor magnitude is $X_{m}, f_{o}$ is nominal system frequency, and $\Psi$ is the phase angle relative to synchronized coordinated universal time (UTC). Frequency drift is one of the most reliable indicators of system disturbance. The frequency and rate of change of frequency are given in equations (2.2) and (2.3).

$$
\begin{aligned}
& x(t)=x_{m} \cos [\Psi(t)] \\
& f_{o}(t)=\frac{1}{2 \pi} \frac{d \Psi(t)}{d t}
\end{aligned}
$$




$$
\operatorname{Rocof}(t)=\frac{d f(t)}{d t}
$$

The PMU measurements are evaluated according to the total vector error (TVE) expression, where the theoretical values of a sinusoid signal vary from the values obtained from a PMU. The standard IEEE Std. C37.118 includes the data transfer protocol for PMUs for power systems. The messaging format with required reporting rates is defined. The locally available measurements are frequency, ROCOF, primary voltages, currents, real and active power flows, and other analog/digital quantities defined by the system operators. The time-synchronized frequency, voltage, active, and reactive power measurements are important to safely operate microgrids.

\subsubsection{Phasor Data Concentrator}

A PDC can be considered as a station in a communication network, where PMUs populate time-aligned measurements. The deployed PMUs in the system send acquired data, with time-stamp information, to the PDC. The data collected from a number of PMUs

are sorted and correlated according to the time-stamp value, which enables comparable real-time monitoring of the system with high precision sampling. The collected data is also stored in a large database system for accurate post-mortem applications, such as fault event monitoring, loss-of-mains, and blackout analysis.

\subsubsection{GPS Clock}

PMUs are used for time referenced critical applications, such as fault event analysis and protection relays, which require highly reliable satellite clocks. Inaccurate time-stamps can cause misdiagnosis of the network and degrading the controllability of the distribution network. The Inter-Range Instrumentation Group (IRIG-B) time code is widely accepted 
for time distribution in substations. In this study, we use a GPS clock with IRIG-B outputs.

Figure 2.8 shows the GPS antenna and clock deployed for this setup.
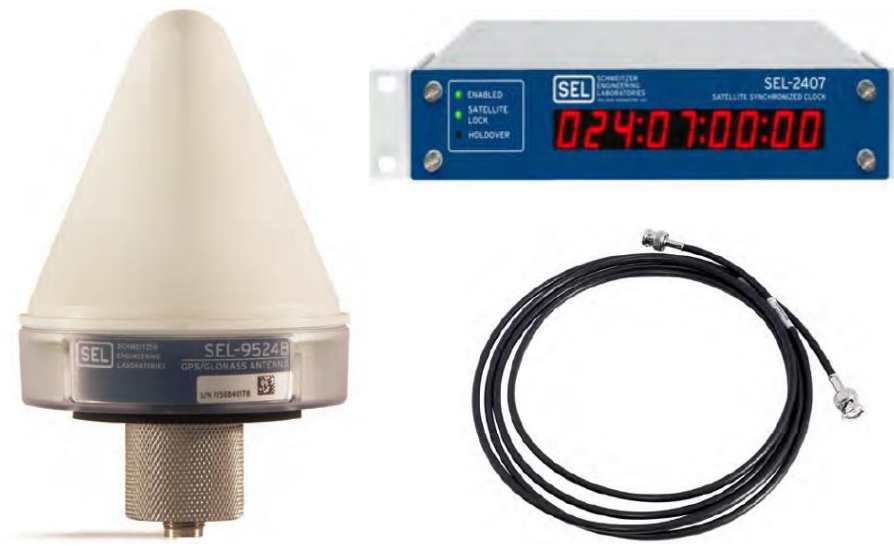

Figure 2.8: GPS clock and antenna.

\subsubsection{Programmable Logic Controller}

Active distribution networks require more field actuator controls, due to DERs, when compared to the passive grid structure. Generator process control and field sequential relay control are some of the applications of PLCs on the field. The SCADA system is capable of providing the interface of the PLCs through built-in communication ports with options for RS485 and TCP/IP. In this study, the RTAC and PLC interface is established with the very common Modbus protocol.

\subsubsection{Real-Time Automation Controller}

Interoperability is one the major challenges facing power system infrastructure, as many new and existing devices need to securely and effectively communicate to each other. RTAC is an industrial automation platform that enables collection and combination of the number of measurements from the system with the ability to support many communication protocols. This platform enables the central controller to communicate with other devices that exist in the network, such as IEDs, PMUs, and PLCs. In this setup, we use some of the 
commonly used industrial communication protocols, such as Modbus, Schweitzer Engineering Laboratory (SEL), and C37.118 synchrophasor protocols. As shown in Figure 2.7, RTAC has an interface with PMUs, PLCs, and the energy meter through C37.118, Modbus, and SEL, respectively.

\subsubsection{SCADA Interface}

A complete human machine interface (HMI) and supervisory control and data acquisition (SCADA) system is necessary to establish network management by distribution network operators. A SCADA system is built to monitor and control the generic microgrid model. The system is running on a webserver, and is available from the internet, with valid credentials. Figure 2.9 shows the main window of the implemented SCADA system.

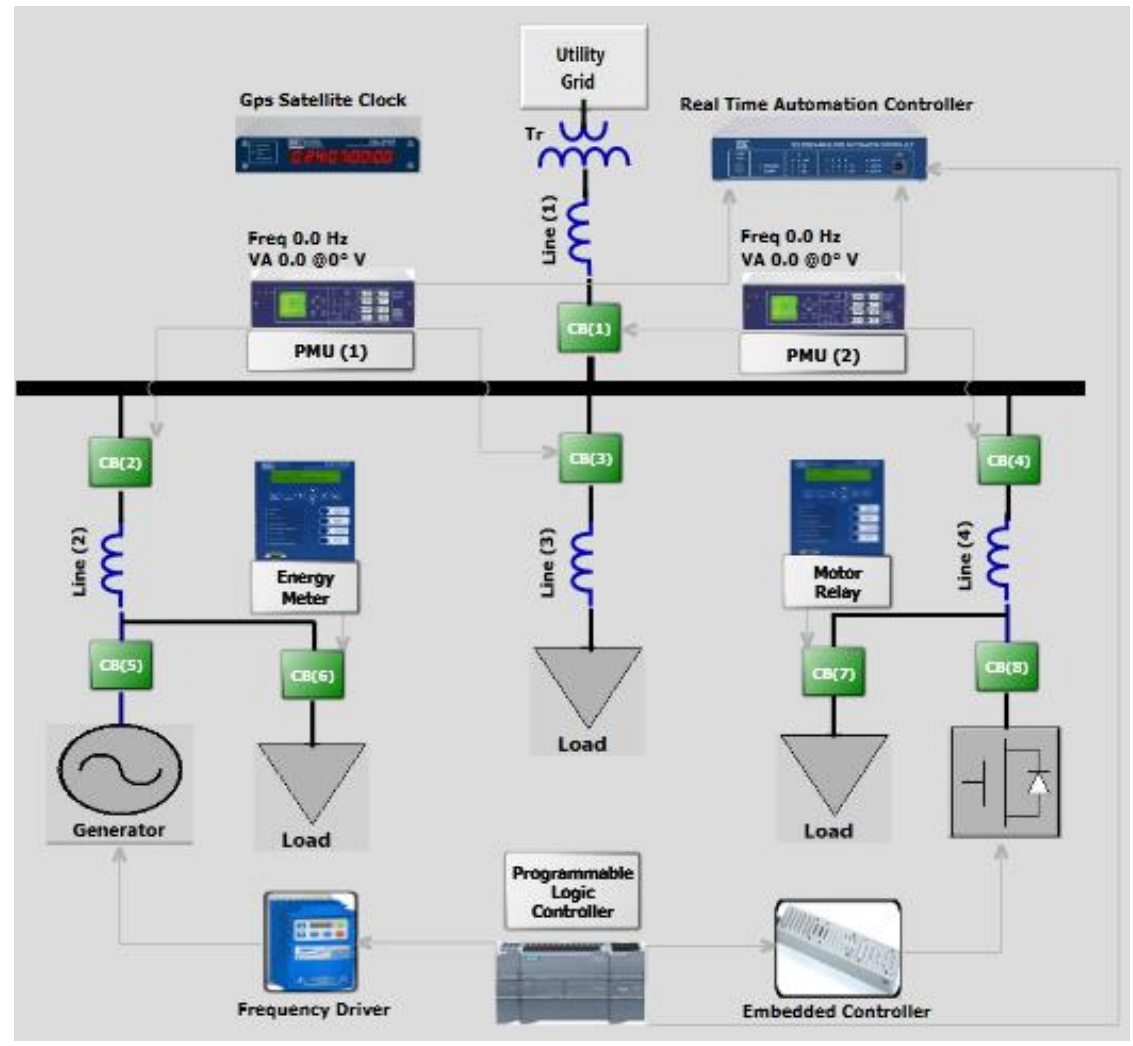

Figure 2.9: SCADA interface of the microgrid. 


\subsubsection{Grid Connection and Information/Communication Architecture}

The grid connection and flexible disconnecting is established using circuit breaker entitled to PMUs and other IED relays. Time synchronized frequency, voltage, current and power measurements are available by PMUs. This has been enabled by the availability of a global positioning system (GPS). Thanks to precise sampled data, the measurements from different system buses are comparable. Two PMUs and an energy meter are deployed on the system as sensor devices. PMUs are capable of measuring two network buses at the same time since they provide two analog measurement channels. Lumped load model is monitored using energy meter and the motor load is protected by the motor protection relay. A phasor data concentrator (PDC) is implemented to measure system parameters through the PMUs. The PMU time-synchronized data update rate is adjusted to 5 messages per second. A PLC is used to enable a load-switching function according to a predetermined load profile. An AC synchronous generator model is used for experiments, which is a 13.8kVA, 60-Hz, 208-V and $1800 \mathrm{rpm}$ synchronous machine. The Automatic Voltage Regulator (AVR) model is a half-wave phase-controlled thyristor-type regulator. Excitation current is derived directly from the generator terminals. The synchronous generator turbine shaft is directly coupled to an induction motor to emulate a governor model. A frequency driver provides the required amount of torque to the induction motor, hence the synchronous generator operates. An inverter-based DER is interfaced to the ac microgrid via a bidirectional ac-dc/dc-ac converter to allow bidirectional power flow between ac and dc sources. A 5-kW programmable dc power supply is used to emulate a typical renewable energy resource. This power supply can be considered as a solar or wind resource. The complete inverter system control is enabled by the digital signal processor 
(DSP)-based platform dSpace Control Desk. A variety of industrial communication protocols have been implemented to acquire field data and to provide information to the actuators. The load is interfaced to the energy meter, which uses Schweitzer Engineering Laboratory (SEL) Fast Message protocol. Modbus is a de facto standard protocol, which is used by many power systems and industrial electronic device vendors. PLC communication is established by Modbus TCP protocol through Ethernet ports. To control the inverter, a raw data exchange between the PC and embedded controller is established with a RS232 serial port. Modbus RTU is used to communicate with the generator through variable frequency drive, which emulates synchronous generator governor model. 


\section{ISLANDING PERFORMANCE AND CONTROL OF HYBRID MICROGRID}

\subsection{Introduction}

In this chapter, islanding performance and control aspects related to hybrid microgrids are investigated. The objective of this chapter is to present a proper voltage and frequency control scheme during cascading islanding by switching appropriate operation modes according to the DER types and locations. First, the frequency and voltage control upon unintetional islanding situations are discussed. Then, the operation mode of DERs are investigated in detail. Finally, the simulation and experimental results are given for cascading unintentional islanding scenarios.

Microgrids offer an essential solution to enable a resilient grid infrasturture since they have the ability to continue operating in case of a utility outage. A microgrid can be viewed as a cluster of DERs, which have a connection to the main utility grid. Microgrids mostly depend on intermittent renewable resources, and this situation may introduce important stability problems, especially during the islanded operation. Minor stability problems can cause cascaded outages, which would result in a large scale blackout unless proper action is taken in time. An effective way to prevent such risks, to ensure robustness and resiliency, is to operate the grid in smaller regions by partitioning the network in case emergency conditions arise.

The islanding process can be classified into two categories: intentional and unintentional. The unintentional islanding process is most likely due to the disconnection of the microgrid when a fault or a utility outage occurs in the system. A microgrid should 
continue to operate by maintaining the voltage and frequency in the islanded area with the appropriate control techniques. A facility island can be assumed as one single source in a microgrid, which is connected through only one circuit breaker to the main utility grid. A secondary island can be considered as multiple DERs and customers' connections to the secondary side of a distribution transformer.

In term of operation, microgrid functionality can be operated in 4 modes: gridconnected, transition-to-island, island, and reconnection. Grid-connected operation occurs when the microgrid is coupled to the main utility grid in order to deliver/receive power to/from the grid. In the grid-connected mode, the frequency and voltage of microgrid can be supported by the strong power system; therefore, a complex regulation control is not necessary. The transition process from the grid-connected to the islanded operation mode should be smooth, without violating power quality indicators, such as voltage and frequency levels. Microgrids should be able to provide the real and reactive power requirements in the islanded area while regulating the voltage and frequency within limits.

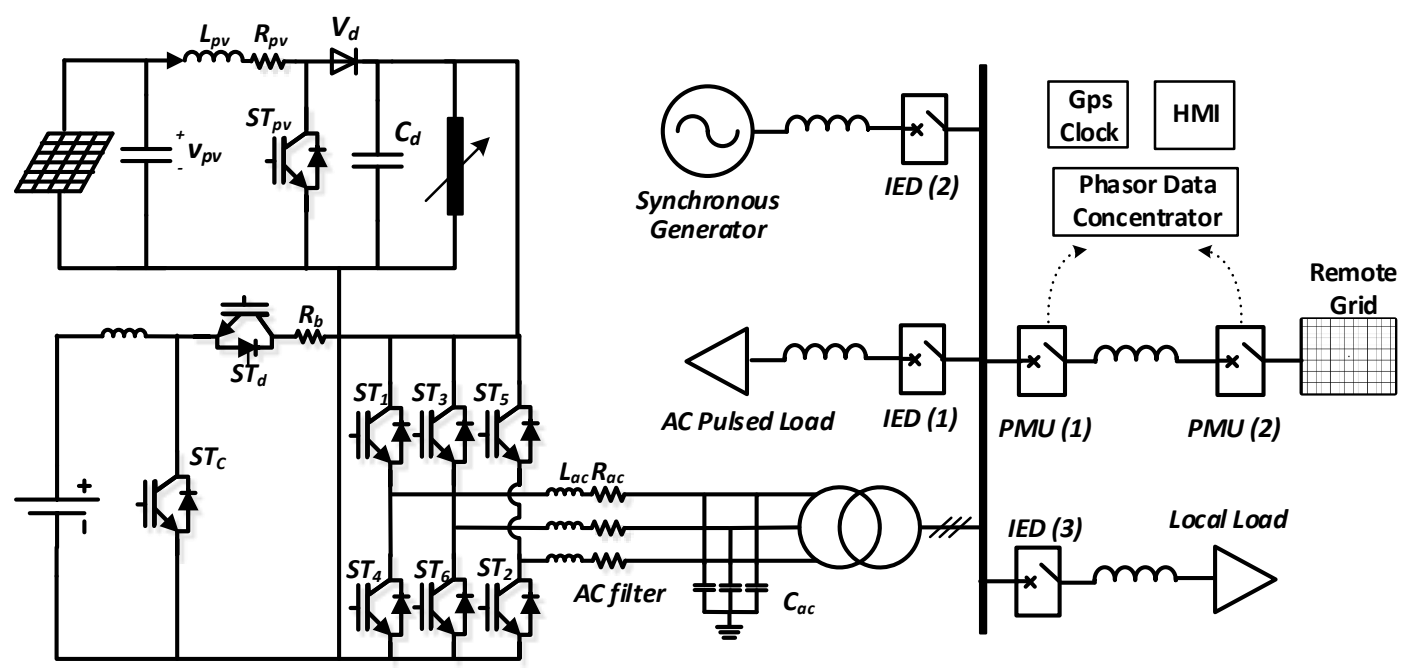

Figure 3.1: Microgrid architecture scheme. 
In order to evaluate the operation of the microgrid, the hybrid microgrid that we have developed is reconfigured, as shown in Figure 3.1. This architecture is composed of DC/DC and AC/DC converters, a synchronous generator, and load models.

\subsection{Control of Islanded Microgrid}

Control of the voltage and frequency subsequent to islanding operation of a microgrid is a major challenge for proper operation. In islanded microgrids, conventional DERs have a slower response to load changes when compared to inverter-based DER due to their high inertia. Inverter-based DERs, which are power electronically interfaced, have a faster response to load changes than the conventional DERs. Most of the DERs cannot connect to the grid directly since the renewable energy generation is mostly based on DC resources, such as solar panels. Therefore, a power electronic interface is required to establish an AC grid connection.

Frequency is directly related to the active power balance in the system. Similarly, the voltage is regulated by reactive power balance. The unintentional islanding process can cause severe problems due to unexpected frequency and voltage stability issues, if either active or reactive power imbalances exist between the microgrid and the main utility grid. Load shedding is one of the easiest solutions to disconnect the required load inside the microgrid to achieve supply and demand balance. However, many systems involve critical loads, where the shortage of electricity is prohibited. In this chapter, it is assumed that the generation capacity is enough to meet the load demand in the islanded area. Frequency and voltage should be kept within limits for the reconnection mode, which is between $59.3 \mathrm{~Hz}$ to $60.5 \mathrm{~Hz}$. 
Although the ultimate goal is to regulate frequency and voltage, the control of the synchronous generator and inverter-based DERs are different. Once the unintentional islanding occurs, the main grid support is no longer available for the microgrid. Operation modes of microgrids are shown in Figure 3.2. According to this scheme, DER should be able to switch operation modes according to pre-determined islanding conditions to regulate frequency and voltage in the islanded area.

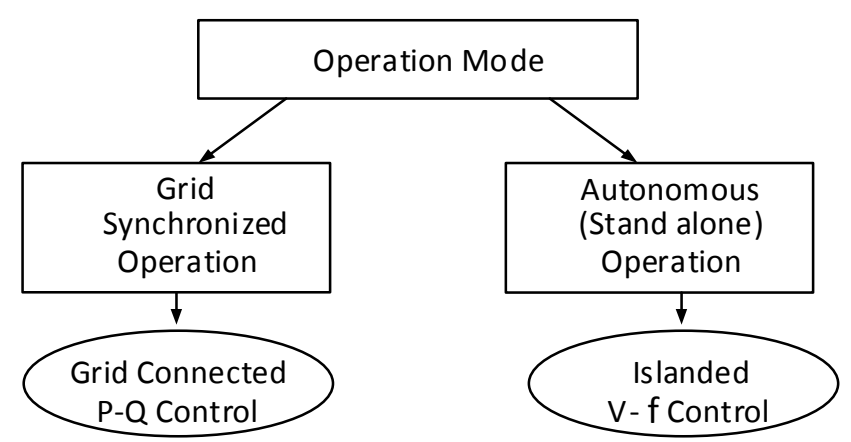

Figure 3.2: Operation modes of microgrid.

If the form of the island is local (facility), where only a single generation unit exists, the DER should be switched to isochronous mode of operation to supply all electrical power of the host facility. Alternatively, if the form of the island is secondary, where multiple generation units exist, a proper load sharing mode should be applied. If the power mismatch is less than what a generation unit can handle, one unit can be selected as an isochronous machine to regulate system frequency and voltage. Otherwise, a droop control can be applied to the generation units for the desired load sharing operation.

\subsection{Operation Mode of DERs}

The major challenge is to decide the appropriate operation mode of each individual DER while taking into consideration intermittent, renewable, and limited fuel resources. In 
the rest of this section, different forms of islanding, involving renewable energy sources (RES) and traditional resources, are discussed.

\subsubsection{Synchronous Generator Grid-Connected Operation}

Synchronous generators can operate in parallel with the grid in P-V (fixed active power and terminal voltage) mode, or $\mathrm{P}-\mathrm{Q}$, constant (fixed active and reactive power) mode. Synchronous generators follow the grid frequency and deliver the required power to the grid by applying necessary amount of torque by generator governor control. In $\mathrm{P}-\mathrm{V}$ mode, the synchronous generator excitation system arranges the current flow from excitation field windings to regulate voltage by generating the required reactive power. Synchronous generators have the capability to operate under-excited and over-excited modes to accommodate reactive power requirements. In P-Q mode operation, the synchronous generator is adjusted to deliver a constant amount of active and reactive power with a predefined power factor to the grid. The only difference between P-V and P-Q mode is that the generation unit contributes to the voltage regulation in the former. The related control block diagram is shown in Figure 3.3.

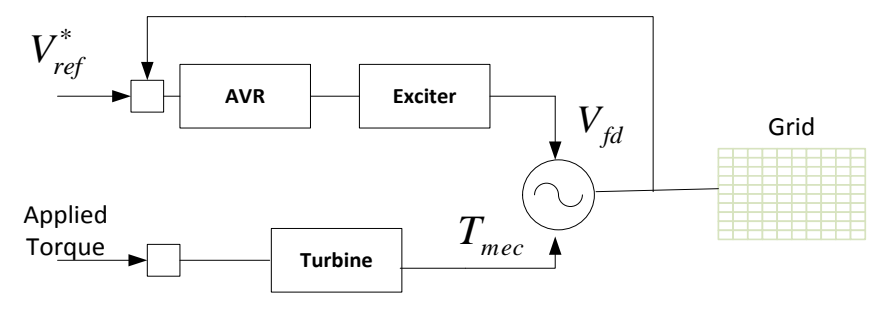

Figure 3.3: Synchronous generator grid connected (P-V) operation.

\subsubsection{Synchronous Generator Islanded Operation}

Subsequent to islanding process, if the synchronous generator is operating in $\mathrm{P}-\mathrm{V}$ or P-Q mode, a power mismatch would appear between islanded microgrid power demand 
and supply. This would cause a stabilization problem, namely frequency drop or increase, related to the power mismatch ratio. For a stable operation, the load and supply should exactly match inside the grid while considering the losses. The voltage inside the microgrid is to be regulated by an automatic voltage regulator if the reactive power demand is within the synchronous generator's limits.

In order to prevent the power mismatch problem, the synchronous generator should be switched to the isochronous mode of operation. In this mode, the generator simply follows the speed or frequency reference of the microgrid. By increasing the applied torque according to the speed error reference, the supplied active power increases. If the microgrid is exporting power to the utility grid, the speed reference turns into a negative value, and the generator then decreases the applied torque to regulate frequency. The V-f operational mode of the synchronous generator, in stand-alone mode, is shown in Figure 3.4 .

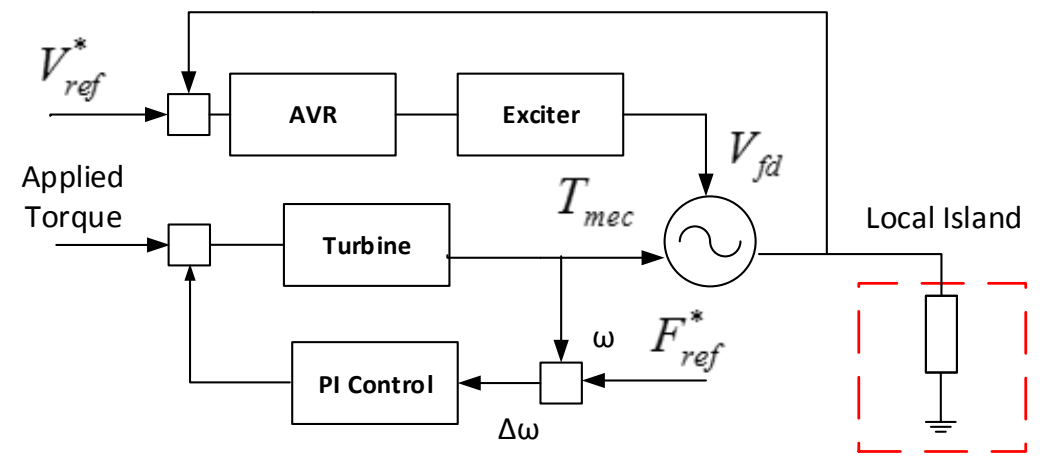

Figure 3.4: Synchronous generator islanded operation.

Synchronous generator motion and excitation equations in the dq0 domain are given as:

$$
\frac{d \delta}{d t}=\omega_{o} \Delta \omega
$$




$$
\begin{gathered}
\frac{d \omega}{d t}=\frac{1}{2 H}\left(T_{m}-T_{e}-K_{D} \Delta \omega\right) \\
\frac{d e_{q}}{d t}=\frac{1}{T_{d o}}\left(E_{f d}-e_{q}-\left(X_{d}-X_{d}^{\prime}\right)\right.
\end{gathered}
$$

$\delta$ is the rotor (load) angle, $\omega_{o}$ is the nominal synchronous speed, $\omega$ is the rotor speed, $H$ is the inertia constant, $K_{D}$ is the damping factor, $e_{q}$ is the transient internal armature voltage, $T_{d o}$ is the direct-axis time constant, $E_{f d}$ is the steady state armature voltage, $X_{d}$ and $X_{d}{ }^{\prime}$ are the direct axis reactance and transient reactance respectively, $T_{m}$ and $T_{e}$, are the mechanical and electrical torques respectively, and $i_{d}$ is the direct axis current. Electrical output torque, terminal active and reactive power:

$$
\begin{gathered}
T_{e}=\frac{V_{d} i_{d}+V_{q} i_{q}}{\omega} \\
P=\omega_{o} T_{e}=V_{d} i_{d}+V_{q} i_{q} \\
Q=V_{q} i_{q}-V_{d} i_{d}
\end{gathered}
$$

$P, Q$ are terminal active and reactive powers respectively.

$$
\begin{gathered}
V_{t}=\sqrt{V_{d}^{2}+V_{q}^{2}} \\
I=\frac{\sqrt{P^{2}+Q^{2}}}{V_{t}} \\
\theta=\tan ^{-1}\left(\frac{Q}{P}\right)
\end{gathered}
$$

The terminal voltage and currents are given as:

$$
\begin{gathered}
V_{d}=X_{q} i_{q}-X_{e} i_{q} \\
V_{q}=-\left(X_{d}-X_{e}\right) i_{d}+X_{a d} i_{f}
\end{gathered}
$$




$$
\begin{aligned}
& i_{d}=\frac{e_{q}-V_{q}}{X_{d}{ }^{\prime}} \\
& i_{q}=\frac{V_{d}}{X_{q}}
\end{aligned}
$$

$i_{d}$ and $i_{q}$ are currents in the $\mathrm{d}$-axis and q-axis. $V_{d}, V_{q}$ are the d-axis and q-axis output voltages. Here, $X_{a d}$ is the d-axis mutual reactance and $i_{f}$ is the field current.

\subsubsection{Inverter-based DER Grid-Connected Operation}

Inverter-based DERs can operate in both grid-connected or standalone mode. If the inverter is operating in grid-connected mode, the voltage and frequency are regulated by the grid; therefore, the inverter is responsible for delivering reactive or active power according to the current control reference generated by a hysteresis-based proportional integral (PI) control. Figure 3.5 shows the block diagram of a grid-connected inverter operation.

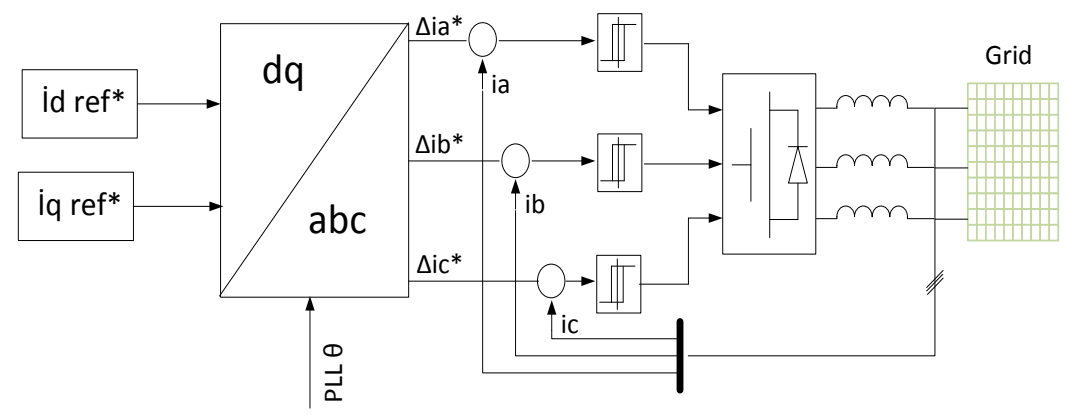

Figure 3.5: Inverter grid-connected operation.

According to instantaneous power theory in the synchronous reference frame, the state space representation of the injected current and the system voltage relations are given below. Here, $V_{g}$ is the grid voltage and, $L_{s}$ and $R_{S}$ represent resistance and inductance of the filter, respectively. 


$$
\begin{gathered}
\frac{d}{d t}\left[\begin{array}{l}
i_{a} \\
i_{b} \\
i_{c}
\end{array}\right]=\frac{R_{s}}{L_{s}} V_{g}\left[\begin{array}{l}
i_{a} \\
i_{b} \\
i_{c}
\end{array}\right]+\frac{1}{L_{s}}\left(\left[\begin{array}{c}
V_{g a} \\
V_{g a} \\
V_{g a}
\end{array}\right]-\left[\begin{array}{c}
V_{a} \\
V_{b} \\
V_{c}
\end{array}\right]\right) \\
{\left[i_{q d 0}\right]=T\left[i_{a b c}\right]} \\
\theta=\omega t+\theta_{0} \\
T(\theta)=\frac{2}{3}\left[\begin{array}{ccc}
\cos (\theta) & \cos (\theta-2 \pi / 3) & \cos (\theta+2 \pi / 3) \\
\sin (\theta) & \sin (\theta-2 \pi / 3) & \sin (\theta+2 \pi / 3) \\
1 / 2 & 1 / 2 & 1 / 2
\end{array}\right]
\end{gathered}
$$

$\omega$ is the angular frequency, $\theta$ is the electrical angle and $\theta_{0}$ represents initial value.

$$
\begin{gathered}
\frac{d}{d t}\left[\begin{array}{l}
i_{d} \\
i_{q}
\end{array}\right]=\left[\begin{array}{cc}
-\frac{R_{S}}{L_{s}} & \omega \\
-\omega & -\frac{R_{S}}{L_{S}}
\end{array}\right]\left[\begin{array}{l}
i_{d} \\
i_{q}
\end{array}\right]+\frac{1}{L_{s}}\left(\left[\begin{array}{l}
V_{g d} \\
V_{g q}
\end{array}\right]-\left[\begin{array}{l}
V_{d} \\
V_{q}
\end{array}\right]\right) \\
S=P+j Q=3 V_{g d} I^{*}{ }_{g d}=3\left(\frac{V_{q}-j V_{d}}{\sqrt{2}}\right)\left(\frac{I_{q}+j I_{d}}{\sqrt{2}}\right)
\end{gathered}
$$

The power injected to the grid for a three-phase system results:

$$
\begin{aligned}
& P=\frac{3}{2} V_{q} I_{q}+I_{q} I_{d} \\
& Q=\frac{3}{2} V_{q} I_{d}-V_{d} I_{q}
\end{aligned}
$$

In grid-connected mode, the AC side can be viewed as an infinite bus; therefore, the deviation of the voltage amplitude and frequency can be ignored. In this case, the bidirectional $\mathrm{AC} / \mathrm{DC}$ converter only needs to regulate the $\mathrm{DC}$ bus voltage. In order to operate in unity power factor, reference $i_{q}$ can be set as 0 . The controller only needs to control the $i_{d}$, which controls the active power flow through the converter. A more detailed control block diagram for the bi-directional AC/DC converter in grid-connected mode is shown in 
Figure 3.7. A two-loop controller is used to regulate the DC bus voltage, as shown in Figure 3.6.

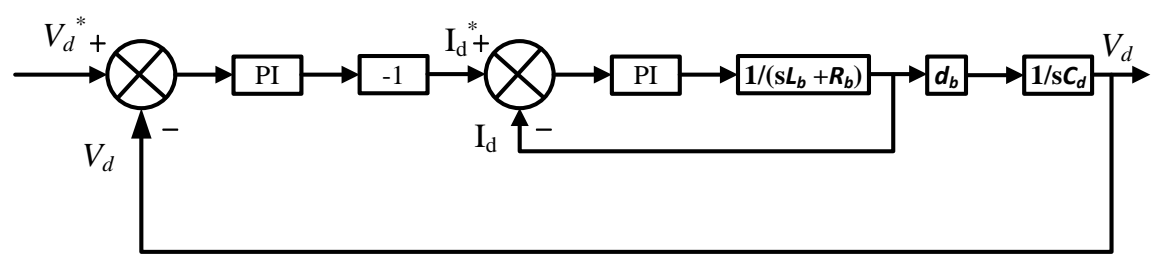

Figure 3.6: Control block for bi-directional DC/DC converter.

Based on the error between the DC bus reference voltage and measured voltage, the outer voltage control loop generates the $i_{d}$ reference, which is used to regulate $i_{d}$ in the bi-directional converter, as shown in Figure 3.6. In $d$-q coordinates, $I_{d}$ is controlled to regulate the active power flow through the inverter, and $I_{q}$ is controlled to regulate the reactive power flow through the inverter. In the AC side, the active and reactive power flow influence the frequency and voltage amplitude, respectively.

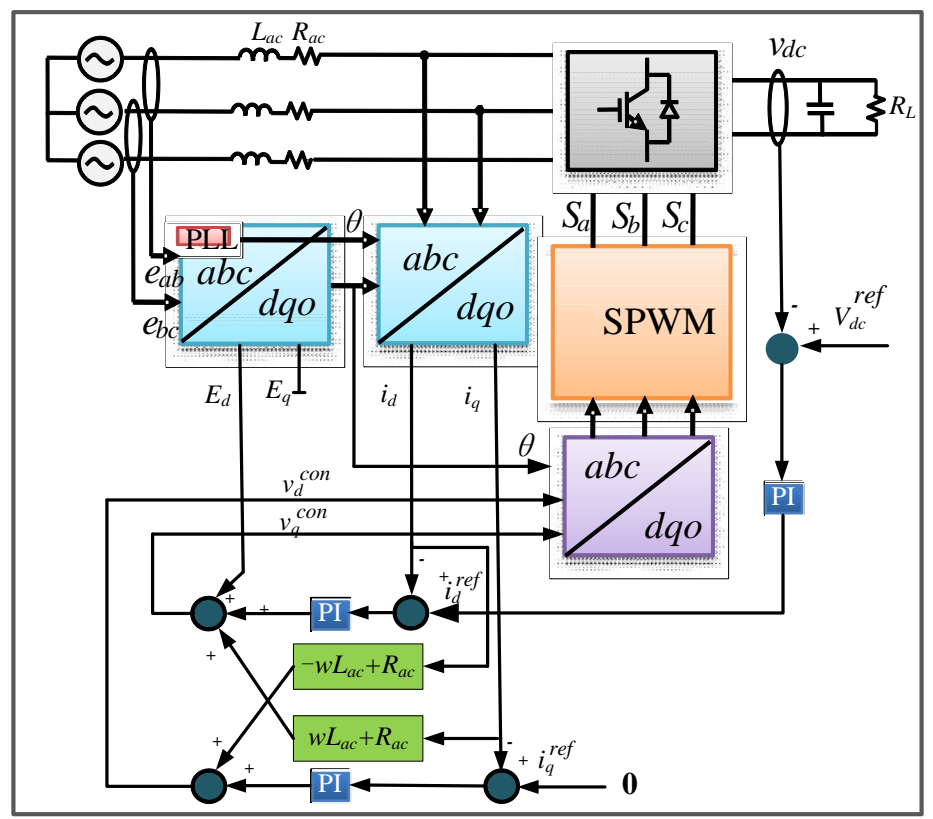

Figure 3.7: Detailed inverter grid-connected operation. 


\subsubsection{Inverter-based DER Islanded Operation}

When the islanding condition is detected, the inverter still operates in gridconnected mode, but the phase lock loop (PLL) fails to operate since the grid frequency reference no longer exist. This situation can cause extreme conditions, and these conditions may interrupt the microgrid's services. In order to improve resiliency and maintain continuous service, the inverter controller must be automatically reconfigured as voltage control upon islanding detection. The reconfiguration process can be triggered by the trip signal from the islanding detection algorithm, which is basically a comparison of the DER and utility phase angle difference.

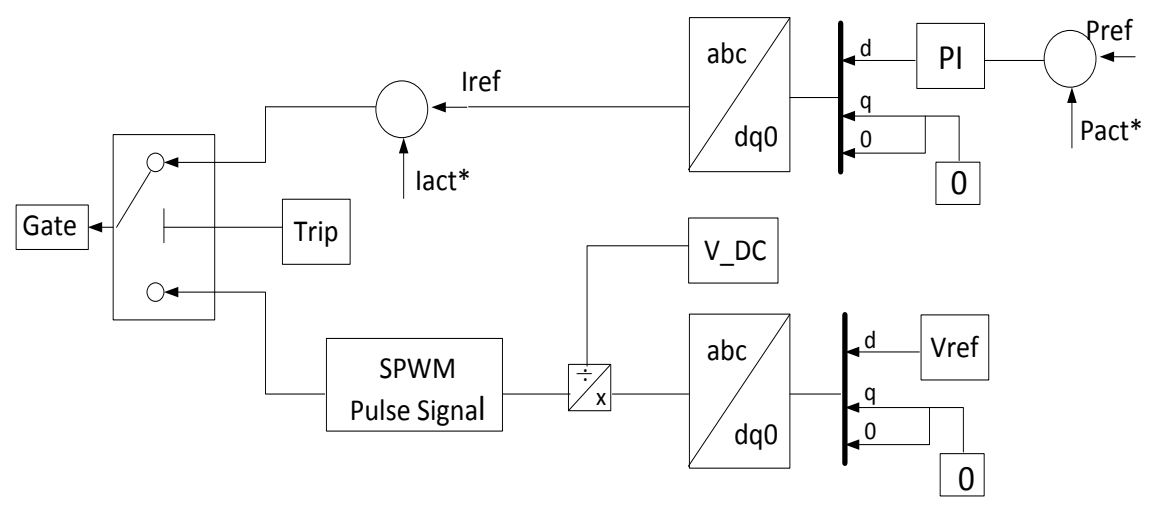

Figure 3.8: Inverter Islanded Operation.

Figure 3.8 shows the block diagram for the proposed reconfigurable converter control algorithm. If the grid voltage signal is no longer available, the inverter cannot track the grid frequency reference. Thus, the control is switched to generate its own internal frequency reference and operate in voltage control mode on the terminal point by the adjusted modulation index. The system's voltage and frequency are regulated by supplying the required amount of active and reactive power to the local load. 
In the three-phase voltage source inverter (VSI) space vector modulation pulsed width modulation (SVPWM) technique, the maximum amplitude of the fundamental phase voltage in the linear region is $m_{a}<1$ is $V_{d c} / 2$, where the modulation index is $m_{a}$. The maximum amplitude of the fundamental $\mathrm{AC}$ output line voltage is:

$$
V_{a b}=m_{a} \frac{\sqrt{3}}{2} V_{d c}
$$

\subsubsection{Inverter-based DER and Synchronous Generator Secondary Island}

In a secondary island situation, multiple DERs are connected to the same system feeder or bus. In this case, one of the generation units should take control to regulate frequency and voltage in the islanded area. Since the inverter has a faster response and the ability to track system change, the inverter-based DER is chosen to operate in isochronous mode. The required amount of active and reactive power is injected from the inverter-based DER in order to cover the power mismatch between supply and demand. In addition to the basic current control strategy, one more control loop is implemented to regulate the frequency and voltage, as shown in equations (3.23) and (3.24). Figure 3.9 shows the block diagram of a secondary island.

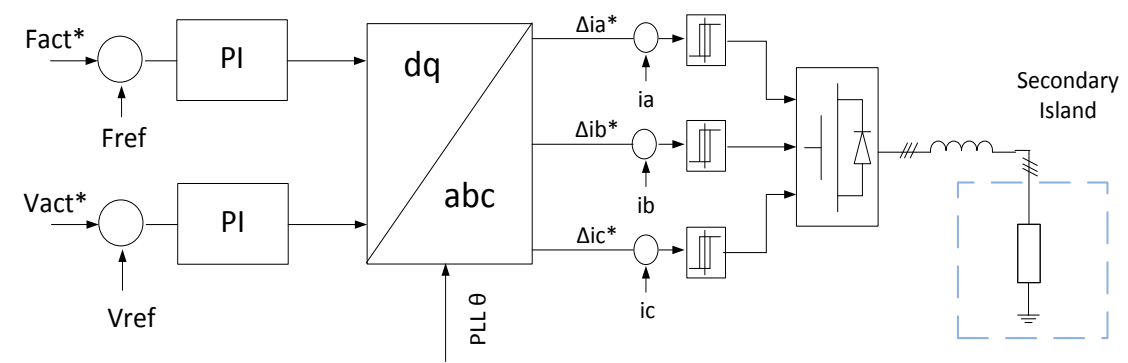

Figure 3.9: Secondary Islanded Operation. 


$$
\begin{aligned}
& i_{q}^{*}=\left(V_{\text {ref }}-V_{\text {meas }}\right)\left(K_{p v}-K_{\text {iv }} / s\right) \\
& i^{*}{ }_{d}=\left(f_{\text {ref }}-f_{\text {meas }}\right)\left(K_{p f}-K_{i f} / s\right)
\end{aligned}
$$

In islanded operation mode, the frequency and voltage amplitude of the three-phase $\mathrm{AC}$ side are volatile. The bi-directional AC/DC inverter is used to regulate the active and reactive power by controlling the $i_{d}$ and $i_{q,}$, respectively. A more detailed control scheme for the bi-directional AC/DC inverter is shown in Figure 3.10. Two-loop controllers are applied for both frequency and voltage regulation. For frequency control, the error between measured frequency and reference frequency is sent to a PI controller, which generates the $i_{d}$ reference. To control the voltage amplitude, the error between the measured voltage amplitude and the reference voltage amplitude is sent to a PI controller to generate the $i_{q}$ reference.

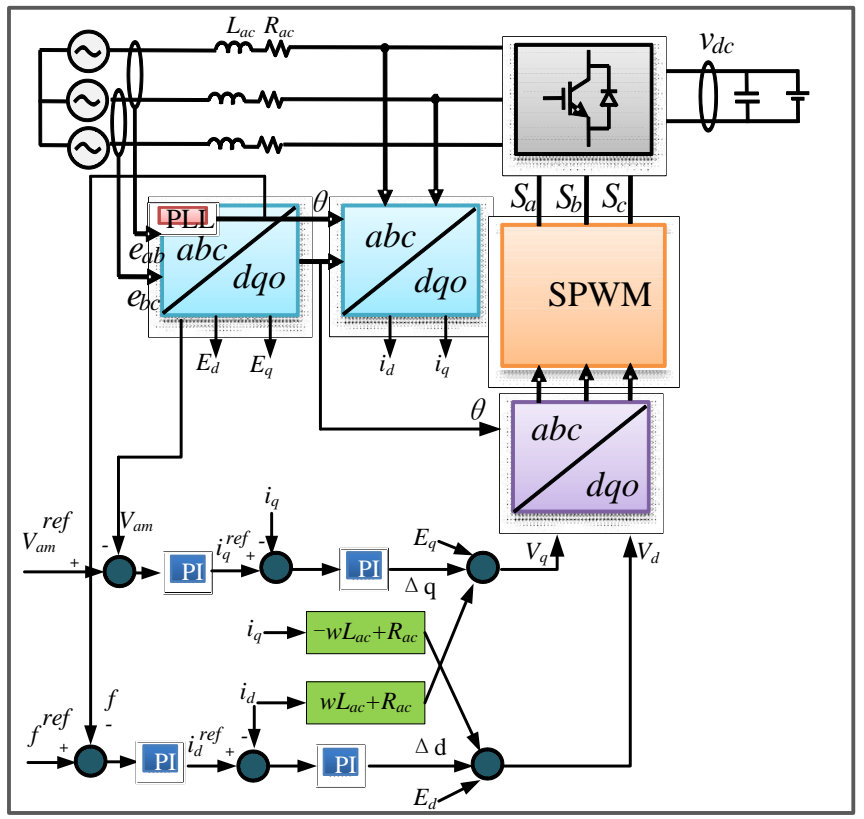

Figure 3.10: Detailed secondary islanded operation. 
Equations (3.25) and (3.26) show the AC side voltage equations of the bidirectional $\mathrm{AC} / \mathrm{DC}$ inverter in $\mathrm{ABC}$ and $d-q$ coordinates, respectively. Where $\left(V_{a}, V_{b}, \mathrm{~V}_{\mathrm{c}}\right)$ are the $\mathrm{AC}$ side voltages of the inverter, and $\left(\mathrm{E}_{\mathrm{a}}, \mathrm{E}_{\mathrm{b}}, \mathrm{E}_{\mathrm{c}}\right)$ are the voltages of the $\mathrm{AC}$ bus. $\left(\Delta_{\mathrm{a}}, \Delta_{\mathrm{b}}, \Delta_{\mathrm{c}}\right)$ are the signals adjusting the PI controller output as an input for the current control loop.

$$
\begin{aligned}
& L_{a c} \frac{d}{d t}\left[\begin{array}{l}
i_{a} \\
i_{b} \\
i_{c}
\end{array}\right]+R_{a c}\left[\begin{array}{l}
i_{a} \\
i_{b} \\
i_{c}
\end{array}\right]=\left[\begin{array}{l}
V_{a} \\
V_{b} \\
V_{c}
\end{array}\right]-\left[\begin{array}{l}
E_{a} \\
E_{b} \\
E_{c}
\end{array}\right]+\left[\begin{array}{l}
\Delta_{a} \\
\Delta_{b} \\
\Delta_{c}
\end{array}\right] \\
& L_{a c} \frac{d}{d t}\left[\begin{array}{l}
i_{d} \\
i_{q}
\end{array}\right]=\left[\begin{array}{ll}
-R_{a c} & \omega L_{a c} \\
-\omega L_{a c} & -R_{a c}
\end{array}\right]\left[\begin{array}{l}
i_{d} \\
i_{q}
\end{array}\right]+\left[\begin{array}{l}
V_{d} \\
V_{q}
\end{array}\right]-\left[\begin{array}{c}
\Delta_{d} \\
\Delta_{q}
\end{array}\right]
\end{aligned}
$$

When the pulse load is connected or disconnected to the AC side, the frequency or voltage amplitude changes. After detecting the deviation using the PLL or voltage transducer, $I_{d}$ and $I_{q}$ reference signals are adjusted to regulate power flow through the bidirectional AC/DC inverter. Because of the power flow deviation, the DC bus voltage is also influenced. The DC bus voltage transistor senses the voltage variation in DC bus, and the bi-directional DC/DC converter regulates the current flow between the battery and the DC bus. At the end, the energy is transferred between the battery and the AC side to balance the power flow.

For further frequency and voltage regulation, a droop control mechanism is implemented for solving the microgrid primary control problem, as shown in Figure 3.11. A synchronous generator and a grid-tie inverter adjust their power output according to the no-load speed setting with respect to the system frequency, as per equations (3.27) and (3.28). The power output for any given system frequency can be controlled. The no-load 
frequency of a given generator can be set to obtain any desired power output according to its droop slope, $R$.

$$
\begin{gathered}
f_{N L}=P\left(f_{N L}-f_{F L}\right) /\left(P_{\max }-P_{\text {min }}\right)+f_{s y s} \\
f_{N L}=-P \cdot R+f_{s y s}
\end{gathered}
$$

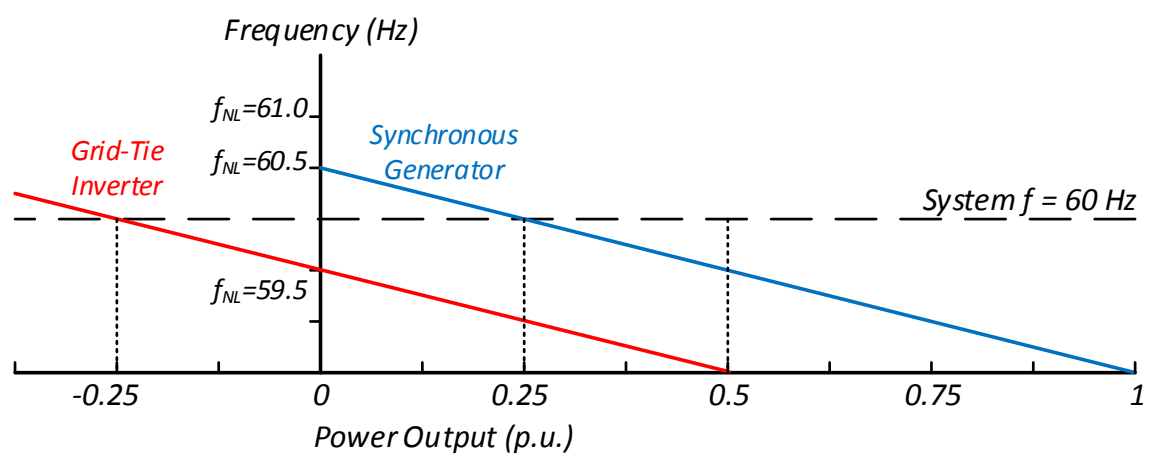

Figure 3.11: Droop slope of generator and inverter.

\subsection{Simulation Results}

The system is modeled in a Matlab/Simulink environment to investigate the possible scenarios. The studied system consists of one conventional synchronous generator model, which can be considered as a diesel or gas turbine-driven generator, and one inverter-based DER model with combined storage. Each DER is modeled for flexible operation, whether in grid-connected or islanded mode. This control structure is achieved by switching the operation modes of the generators from (P-Q) mode to (V-f) mode. The synchronous generator is equipped with excitation, AVR, and governor systems. Both DERs are coupled to the main utility through circuit breakers. Each generation unit has a 
local load of $250 \mathrm{~W}$ and $450 \mathrm{~W}$, respectively, while directly coupled to each DER. The utility grid is connected to the microgrid through a 50kVA $11 / 0.208 \mathrm{kV}$ transformer.

\subsubsection{Grid Connected Operation}

In this case, the synchronous generator and the inverter-based DER are operating in the P-Q grid-connected mode. The frequency and voltage are regulated by the main grid. Figure 3.12 shows the operating conditions prior to islanded operation. Since the synchronous generator is equipped with AVR, the voltage on the generator bus is regulated to a constant reference point through an excitation system.
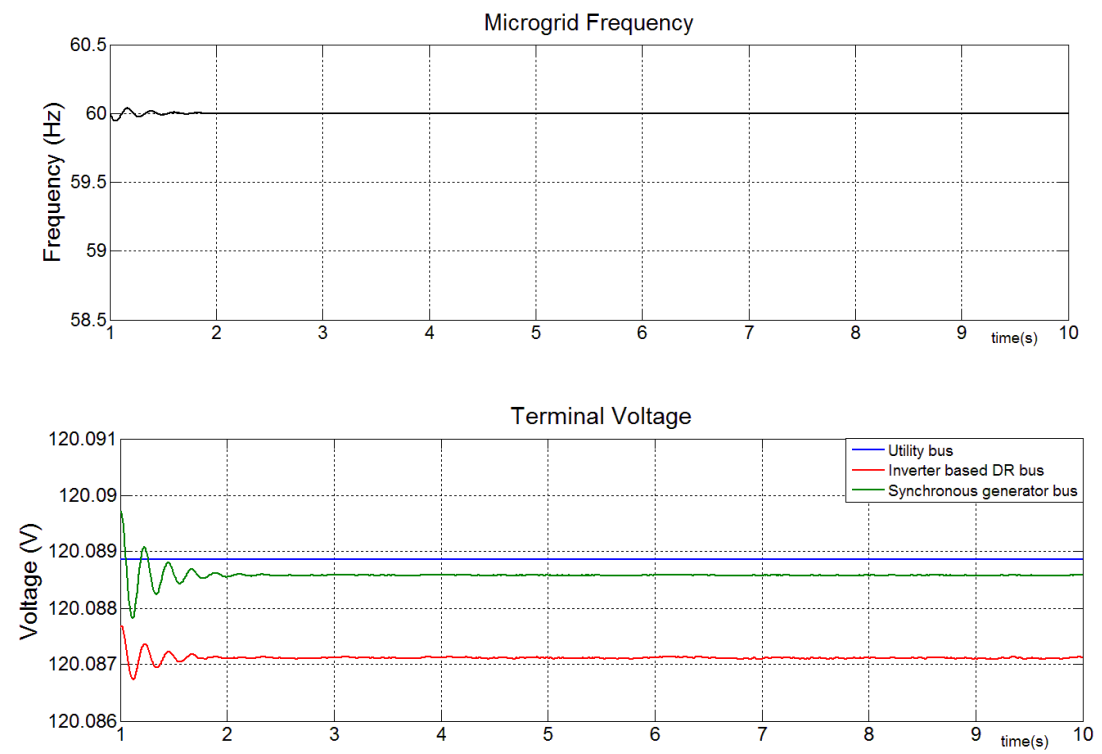

Figure 3.12: Grid connected operation.

\subsubsection{Secondary Islanding Operation}

At the $5^{\text {th }}$ second, a secondary islanding case is created by opening the circuit breaker at the grid's CCP. The resulting island consists of a synchronous generator, an inverter-based DER, and local loads. Figure 3.13 shows the system's frequency and voltage 
collapse as expected, and an instant power mismatch occurred between generation and system load.
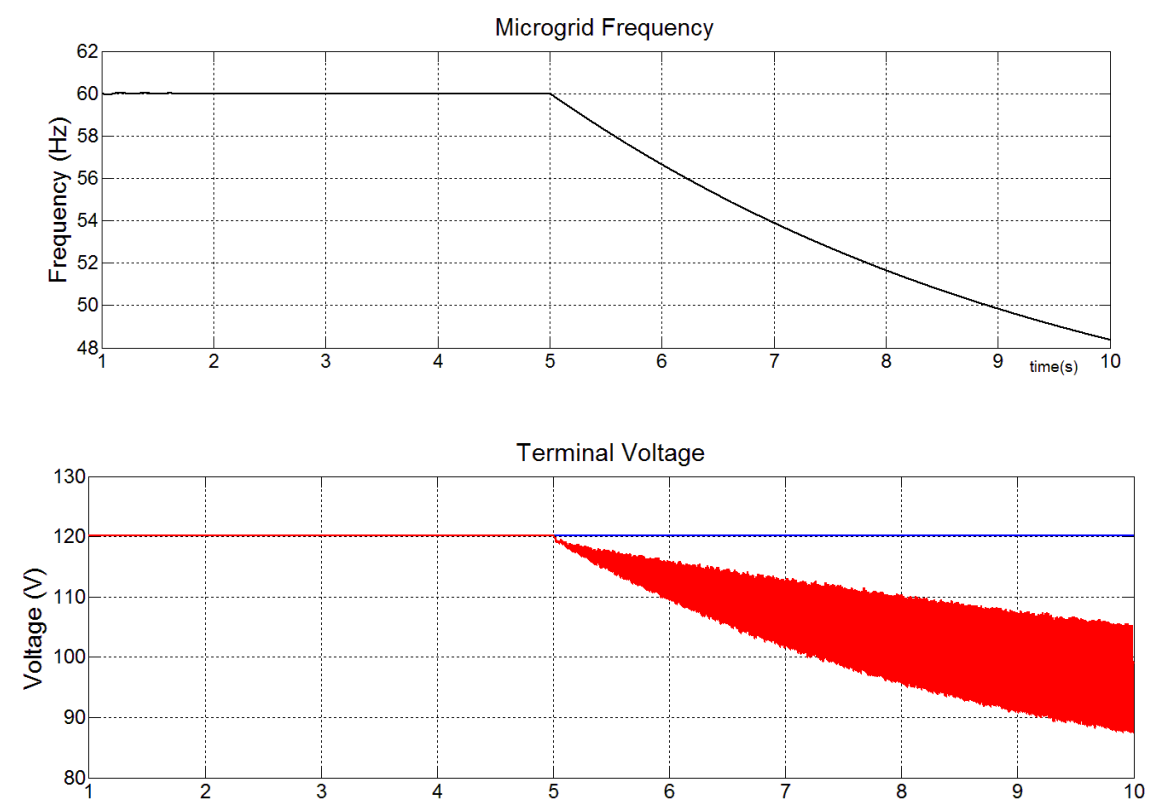

Figure 3.13: System collapse.

If an unintentional islanding protection method is not enabled, due to this mismatch between generation and demand, the system is subject to a frequency and voltage collapse. The frequency measured from the system bus starts to drop gradually, depending on the system inertia. This results in a tripping of the generator due to under frequency, phase angle difference, or ROCOF relays. The frequency drift of the island, with respect to power mismatch, is given in equation (3.29). The system inertia and mismatch ratio are the main effects, which accelerates the frequency change rate during the islanding process.

$$
\frac{d f}{d t}=\frac{\Delta P}{2 H G} f
$$

$\Delta P:$ Power difference between load and generation

$H$ : Moment of inertia generator/system 


\section{$G$ : Rated generation capacity of the generator}

The same scenario is repeated by adding the proposed islanding control of enabling automatic switching of the inverter-based DER operation mode from (P-Q) to (V-f). This is enabled by the islanding detection relay modeled in the simulation environment. Under frequency, phase angle difference, and ROCOF methods are used to enable islanding detection while the synchronous generator remains in the (P-Q) mode. Figure 3.14 shows the measured frequency, voltage, and active power from each unit.
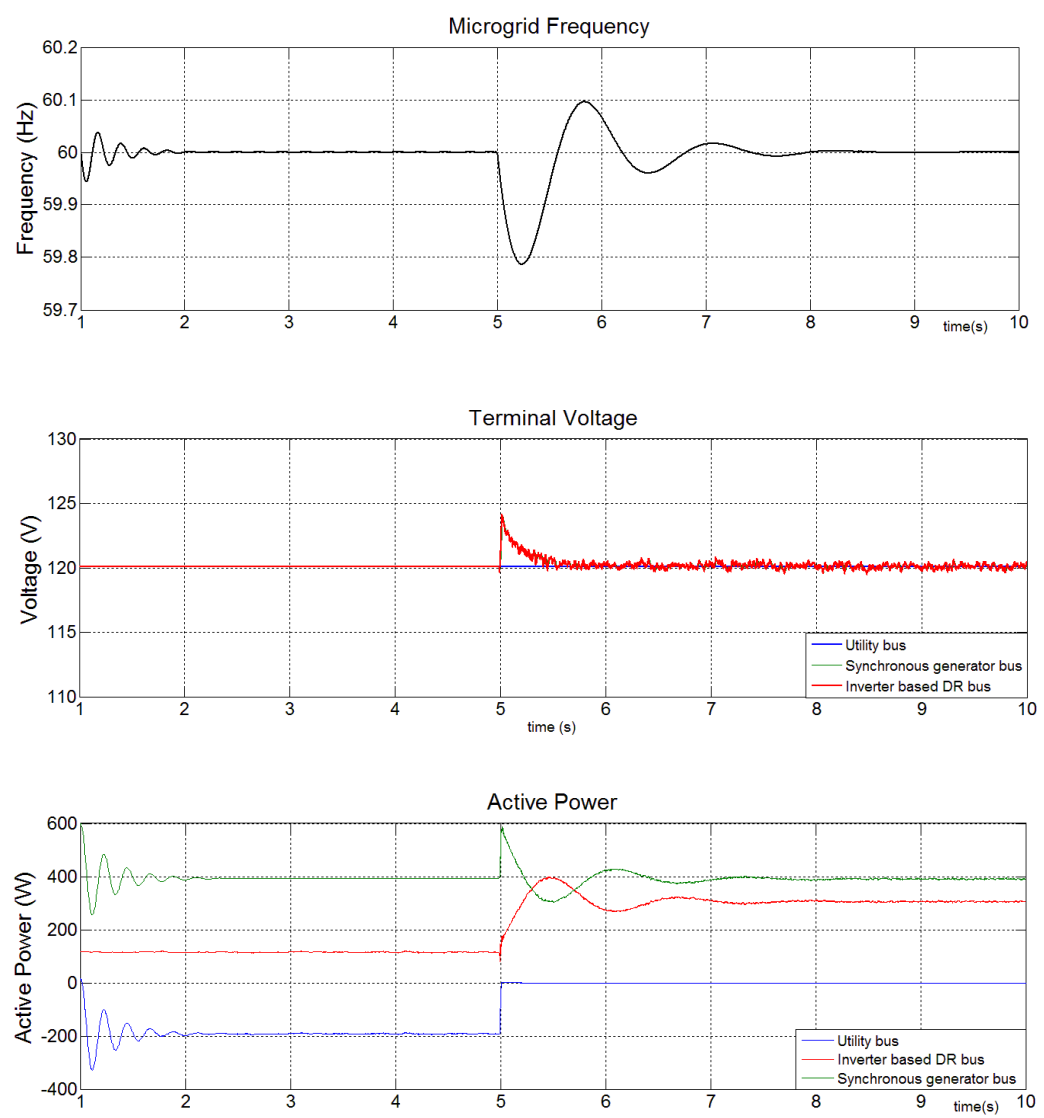

Figure 3.14: Controlled Secondary Islanding.

From observation, we see that the system frequency experiences an oscillation during the transition, which eventually settles to the reference value. The terminal voltage experiences temporary spikes during transition as well, and it settled within the limits due 
to reactive power control inside the island. The delivered active power from the synchronous generator remains the same, and the power drawn from the grid becomes zero due to the disconnection. The inverter-based DER then starts to generate the required amount of active power to keep the frequency within the limits.

\subsubsection{Facility Islanding Operation}

In this case study, while the system is operating in secondary island mode, two separate local islands are formed by opening two circuit breakers on the CCP of the synchronous generator and the inverter-based DER. The synchronous generator is switched to speed (frequency) control mode. The required amount of power supplied is adjusted by applying torque to the generator shaft, which is regulated by the speed governor, according to the frequency error input. In the inverter based DER local island control, neither the grid nor the synchronous generator frequency signal is available for PLL to track; therefore, the inverter-based DER operation mode is switched from the (V-f) mode to voltage control. During the transition, the generator bus frequency experienced a high overshoot since the generated power was more than required. By employing speed control regulation, the generated power was forced down to match the local load requirement, and the frequency settled around the nominal value. Unlike the synchronous generator, the generated power by the inverter-based DER was less than the local load demand, causing the power drawn from the grid to remain at zero. The terminal voltage of the synchronous generator was adjusted by the generated reactive power and AVR control. The predefined modulation index determined the terminal voltage of the inverter-based DER, as illustrated in equation (3.22). Figure 3.15 shows the measured frequency, voltage, and active power for local islanding. 

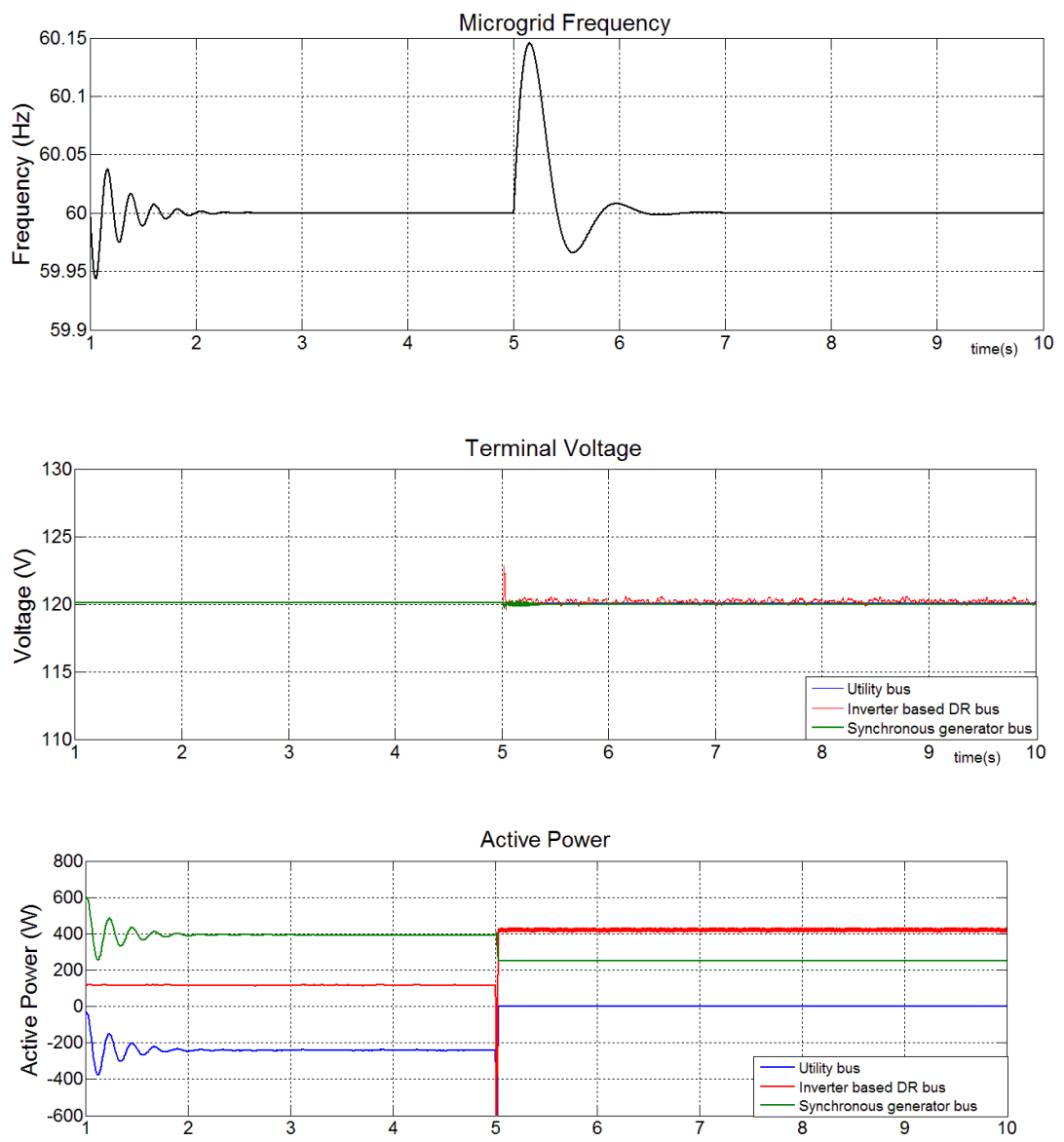

Figure 3.15: Controlled local islanding.

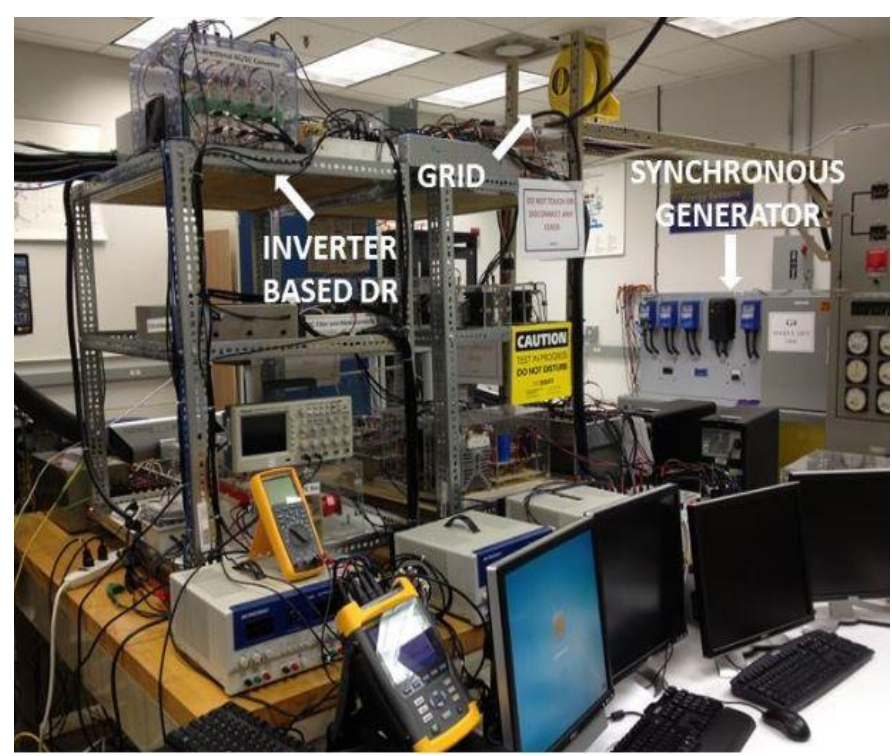

Figure 3.16: Microgrid setup for islanding. 


\subsection{Experimental Results}

The same model is implemented on the microgrid presented in the previous section.

Figure 3.16 shows the hybrid microgrid structure used for real-time experiments. Table 3.1 presents the hybrid microgrid system parameters.

Table 3.1: Microgrid system parameters.

\begin{tabular}{lll}
\hline \hline Symbol & \multicolumn{1}{c}{ Description } & Value \\
\hline$C_{p v}$ & Solar panel capacitor & $100 \mathrm{uF}$ \\
$L_{p v}$ & Inductor for solar Panel boost converter & $5 \mathrm{mH}$ \\
$C_{d}$ & DC bus capacitor & $6000 \mathrm{uF}$ \\
$L_{a c}$ & AC filter inductor & $1.2 \mathrm{mH}$ \\
$R_{a c}$ & Inverter equivalent resistance & $0.3 \mathrm{ohm}$ \\
$L_{b}$ & Battery converter inductor & $3.3 \mathrm{mH}$ \\
$R_{b}$ & Resistance of $L_{b}$ & $0.5 \Omega$ \\
$f$ & Rated AC grid frequency & $60 \mathrm{~Hz}$ \\
$V_{d}$ & Rated DC bus voltage & $300 \mathrm{~V}$ \\
$V m$ & Rated AC bus p-p voltage (rms) & $208 \mathrm{~K}$ \\
$n 1 / n 2$ & Transformer ratio & $1: 1$ \\
\hline \hline
\end{tabular}

\subsubsection{Grid Connected Operation}

In this experiment, the synchronous generator and inverter-based DER synchronize with the grid to supply some portion of local load demand while the remaining power was drawn from grid, as presented in the simulation scenario. Figure 3.17 shows the system frequency and active power measurement on the synchronous generator bus. The system 
frequency is $60 \mathrm{~Hz}$, the delivered active power from synchronous generator is around 400 $\mathrm{W}$, and power drawn from the grid is around $250 \mathrm{~W}$.

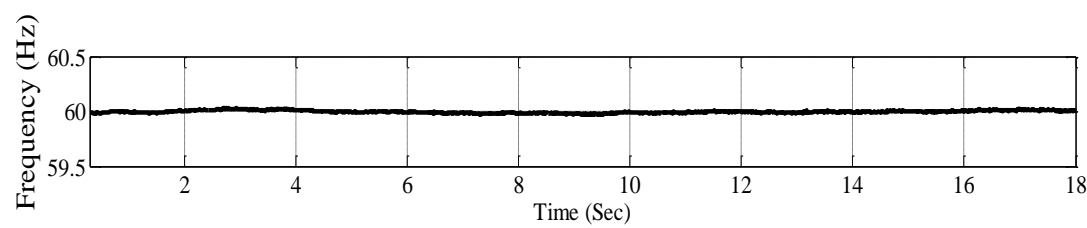

(a)

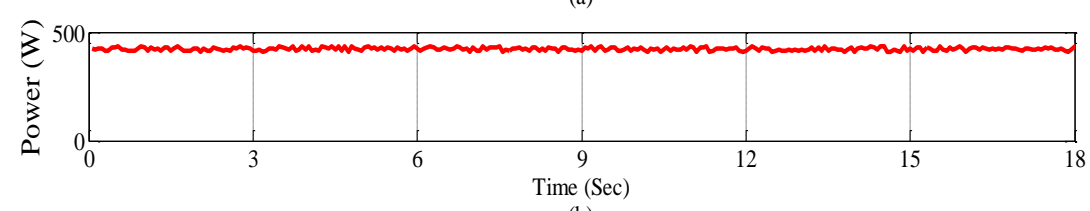

(b)

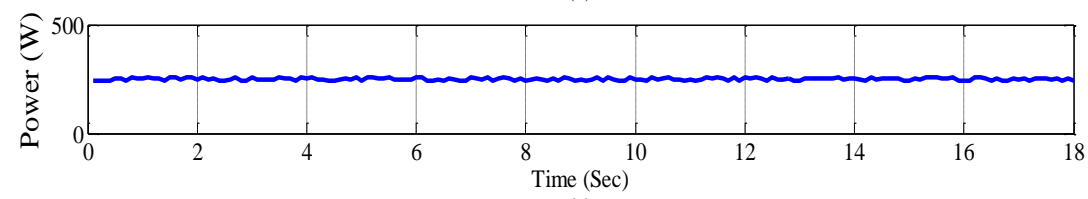

(c)

Figure 3.17: Synchronous generator grid connected operation in experiment. (a) Synchronous generator bus frequency; (b) synchronous generator active power injection to grid (c) active power drawn from grid.

Figure 3.18 shows the active power injected to the grid by the inverter is around $110 \mathrm{~W}$ and the terminal voltage is constant at $120 \mathrm{~V}$.
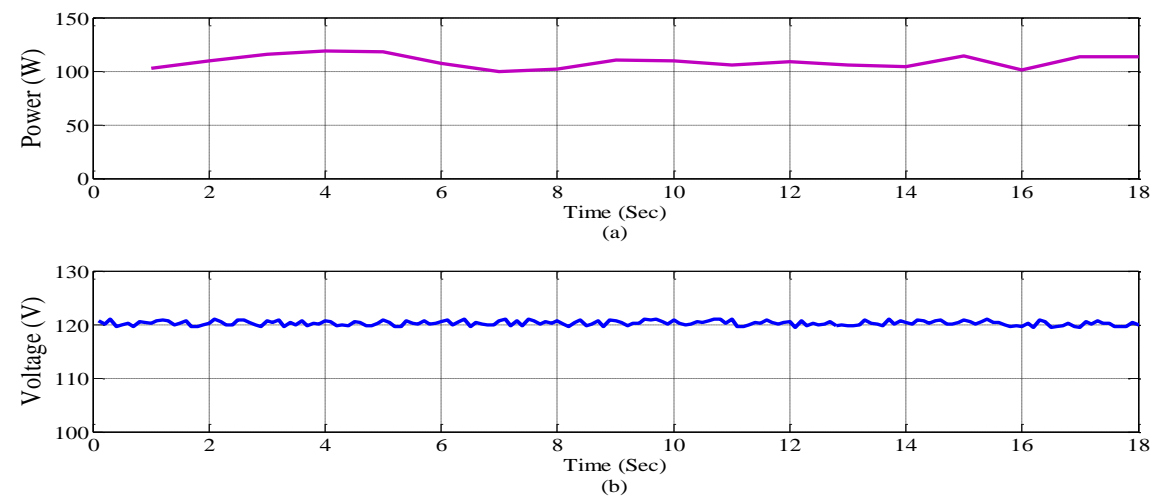

Figure 3.18: Inverter based DER grid connected operation in experiment. (a) Active power supplied by the inverter; (b) terminal voltage. 


\subsubsection{Secondary Islanding Operation}

At the $10.5^{\text {th }}$ second, the secondary island is created by opening circuit breaker between the external grid and the microgrid. Islanding is detected when frequency drops to a defined value, which triggers the switching inverter-based DER to operate in isochronous mode. The inverter-based DER is assigned to operate in isochronous mode to regulate system stability by increasing the amount of active and reactive power to regulate system frequency and voltage. The secondary island experiences a deep frequency drop when utility support is not available. Figure 3.19 (a) shows the recovery of system frequency change and settling on a constant acceptable limit.

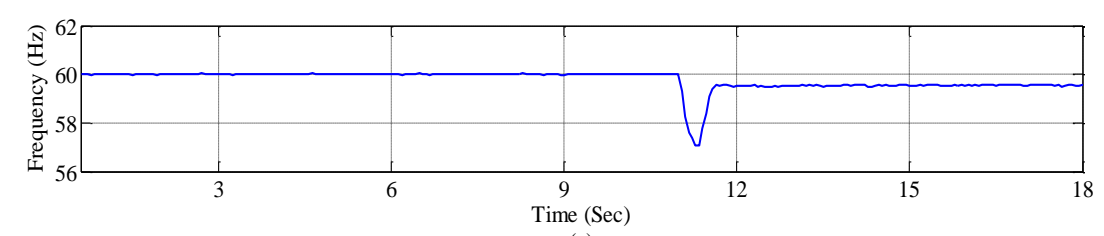

(a)

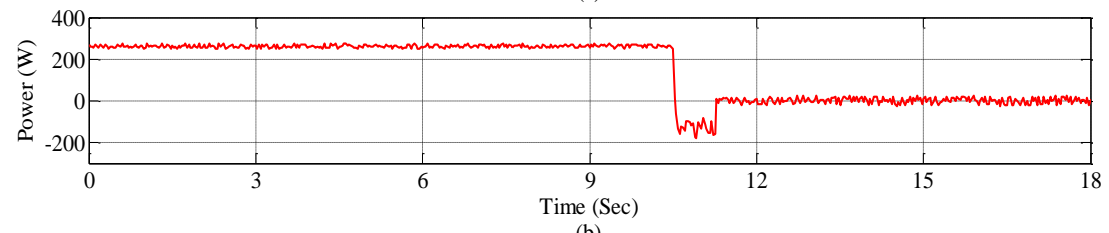

(b)

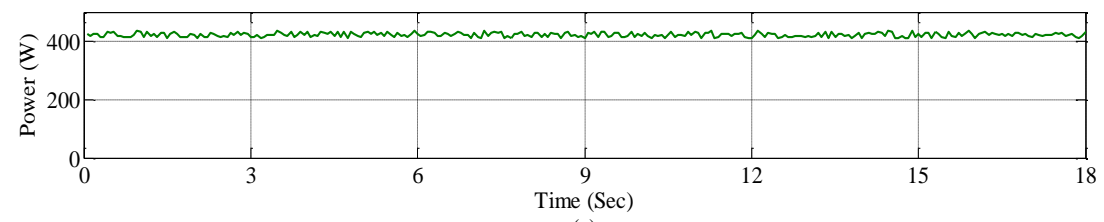

(c)

Figure 3.19: Secondary island grid bus in experiment. (a) Bus frequency; (b) active power drawn from grid (c) active power generation of the synchronous generator.

Frequency decreases to $57 \mathrm{~Hz}$ during the detection and recovery process while frequency is regulated gradually up to $59.5 \mathrm{~Hz}$, which is within the accepted limits according to IEEE 1547.4. Figure 3.19 (b) shows that the active power exchange with the grid dropped to zero which shows that utility support is no longer available. The active 
power generated by the synchronous generator remains almost constant at $420 \mathrm{~W}$, as shown in Figure 3.19 (c).
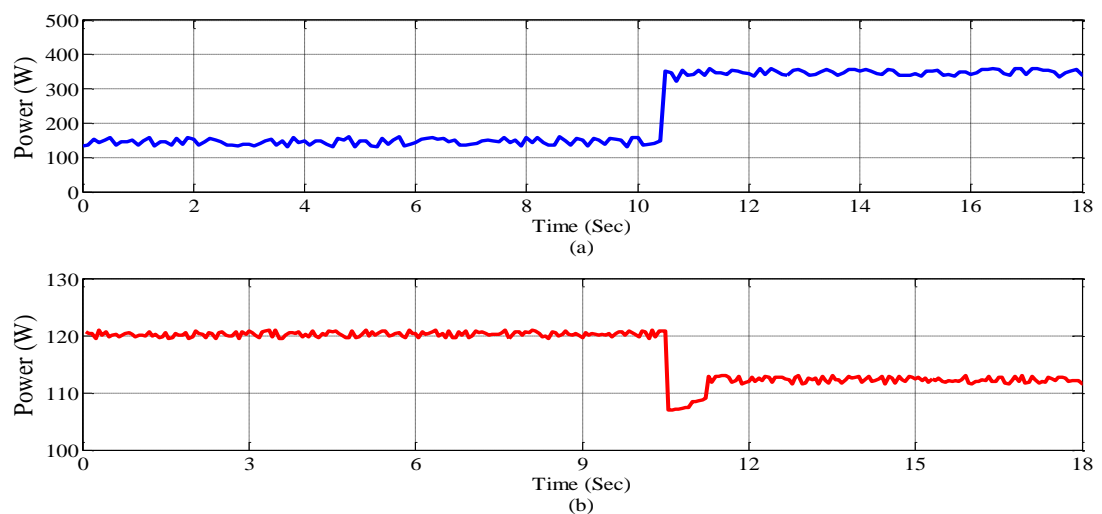

Figure 3.20: Secondary islanding inverter based DR bus in experiment. (a) Active power exchange during transition to islanded operation; (b) terminal voltage.

The inverter based DER takes responsibility for recovery. Figure 3.20 shows the power system values on the inverter-based DER bus. The active power injection increases from $150 \mathrm{~W}$ to $350 \mathrm{~W}$, and the voltage drops and settles around $112 \mathrm{~V}$. It is shown that the inverter is regulating the voltage by balancing the active and reactive power in the system.

\subsubsection{Facility Islanding Operation}

Local islands are created for synchronous generator and inverter-based DER. There is no active power injection from the external grid (the main grid CCP is kept open), and the synchronous generator gets separated from the inverter-based DER. Once the local islanding is detected, the synchronous generator is then switched to frequency regulating mode. At this point, each generation unit starts to supply its own local load. The local load for synchronous generator in this case is around $250 \mathrm{~W}$. The synchronous generator 
decreases the generation from $400 \mathrm{~W}$ to $250 \mathrm{~W}$, as shown in Figure 3.21 (a). This load rejection is associated with frequency increase, and Figure 3.21 (b) shows that the generator frequency is increased at the moment of islanding from 59.6 to $60.2 \mathrm{~Hz}$, where it settles at this value for the remaining operation period. The inverter-based DER local island is created in the same sense. Figure 3.22 shows the performance of the new created island. It can be seen, from Figure 3.22 (a), that the generated active power from the inverter increases from $250 \mathrm{~W}$ to $450 \mathrm{~W}$ to meet the local load requirement. The fast response of the inverter is obvious due to its very low inertia. Figure 3.22 (b) shows that the frequency is regulated to the nominal value of $60 \mathrm{~Hz}$. During the transition period, the voltage drops to $107 \mathrm{~V}$ and is recovered again to be settled around $111 \mathrm{~V}$. In this scenario, the inverter succeeded in regulating the frequency and met the load demand by the local load.

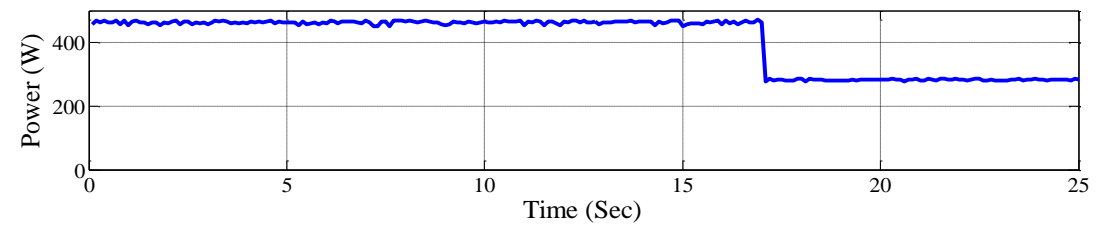

(a)

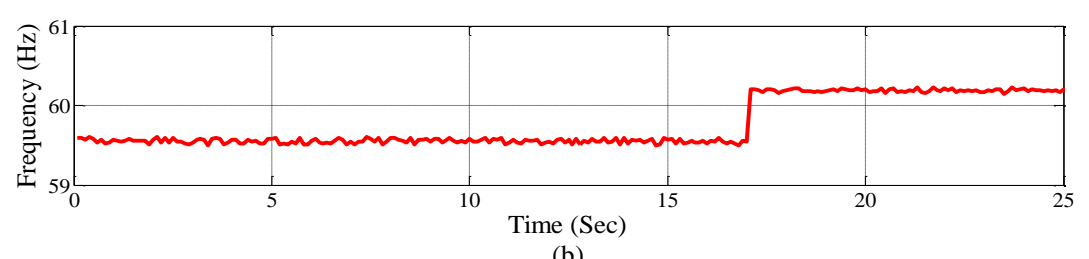

(b)

Figure 3.21: Facility Island of synchronous generator in experiment. (a) active power exchange during transition to islanded operation; (b) generator bus frequency. 


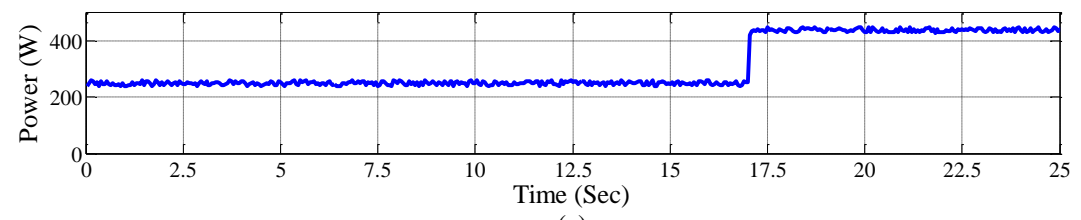

(a)

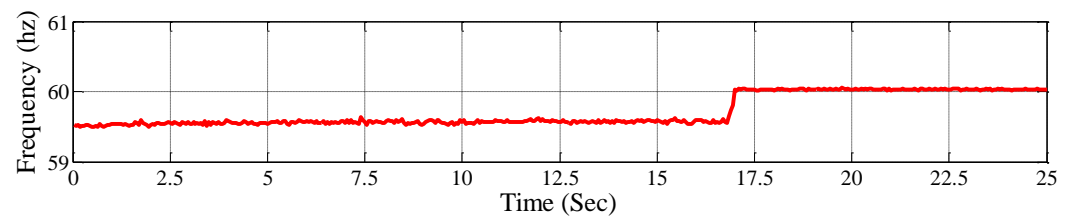

(b)

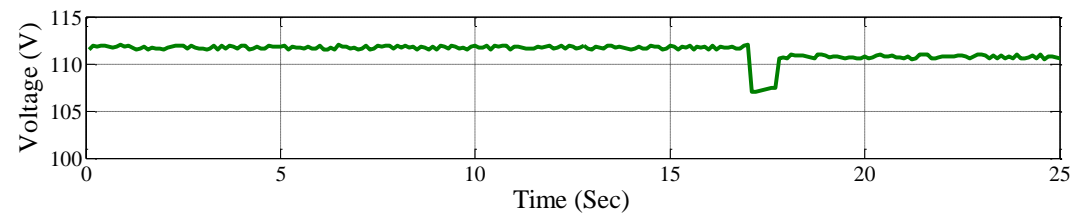

(c)

Figure 3.22: Facility Island of inverter based DER in experiment. (a) Bus frequency; (b) active power exchange during transition to islanded operation; (c) terminal voltage.

\subsection{Summary of the Results and Conclusion}

In this chapter, a control scheme was developed to enable continuous operability of microgrids that comply with the IEEE 1547.4 grid code. A realistic real-time study is developed to examine the sustainable operability of hybrid microgrids upon unintentional islanding situations, which complies with IEEE 1547.4 grid code. The controls for the various islanding structures are investigated, such as local and secondary islands. Synchronous generator and inverter based DER are also studied. The results of the simulation and experiments show that with proper control methods, it is possible to continue operation upon unintentional islanding. 


\section{SYNCROPHASOR ASSISTED CONTROL OF HYBRID AC/DC MICROGRID}

\subsection{Introduction}

This chapter presents real-time synchrophasor assisted microgrid applications using the developed microgrid setup. The focus of this chapter is motivated by the conventional remote terminal unit (RTU) based automation approaches that suffer from slow measurement update and lack of time-stamp values. In this chapter, a time-synchronized control approach is proposed for a deployable hardware-based microgrid involving a synchronous generator and an inverter-based DER. Protection event analysis, islanding detection, re-synchronization, and online remote generation unit dispatch studies are realistically demonstrated. Furthermore, a hierarchical frequency and voltage control is investigated, as well as performance monitoring under pulsed loading conditions.

The distribution networks are nowadays in an on-going change to become similar to transmission networks due to bidirectional energy flow as a result of high penetration of DERs. Thus, the power system is becoming active and more complicated compared to the existing hierarchical grid structure. The ADN concept mainly seeks solutions to enable reliable and efficient operation of DER integrated distribution networks. While system operation becomes active, in the meantime, the aging network structure is rehabilitated by replacing or adding new components into the system, such as PMUs. Such new components enable power system operators to maintain reliable and efficient operation of future smart grids. 
A PMU, which is commonly referred to as a synchrophasor, is one of the most important measurement devices for a power system. PMUs are the power system components, which enable control centers to collect synchrophasor measurement from the network. In order to meet advanced control requirements of ADNs, the measurements from the entire network should be collected in a fast and time-synchronized way so that they can be comparable. A common GPS time source is used to synchronize voltage and current waveforms.

Microgrids are small scale of decentralized electricity networks featuring more complex structure compared to large generation plants. A microgrid may consists of various types of generation units and local loads. Synchronous generators, induction generators and inverter-based DERs are the main types of generation units in a microgrid. As the penetration level of DER increases, concerns regarding the stability and interactions between units are becoming more important. The conventional control and management of the distribution grid, where only voltage magnitudes are measured and utilized at the control center, would undermine these new dynamics and may potentially lead to severe complications in grid operations [67]. This situation results in growing interest to utilize synchrophasors in distribution system applications.

PMU applications in distribution networks has been the subject of increasing interest by many researchers and in the industry [68]. The current trends and possible applications of PMUs in distribution systems vary [69], such as state estimation, dynamic monitoring and fault detection. In previous works, a simulation and training system for ADNs was established to investigate possible scenarios [70]. Simulation studies were carried out to assess dynamic performance of PMU applications [71]. However, simulation 
studies cannot fully address actual system behavior of PMUs, since the simulation is carried out using exact simulation time, neglecting communication delays and missing data.

\subsection{Synchrophasor Application in Microgrid}

Unlike RTU based automation approaches, in the following sections, it has been shown that PMUs enhance the microgrid automation with a fast data frame update, timestamped phasor values with amplitude and phase angle measurements.

\subsubsection{Synchrophasor-based Islanding Detection}

For an accurate phase angle comparison, the phasor measurements should be correlated by means of time-stamp values with a high data frame rate. Thus, PMU measurements can be effectively used to determine whether a microgrid is islanded or not. ROCOF, phase angle difference and over/under frequency are the most accurate indicators for islanding detection. The frequency drift in the islanded area is related to power mismatch and system inertia. Since microgrids have very low inertia, during the load changes the frequency drift has larger values. The frequency drift of the island, with respect to power mismatch, is given in equation (4.1).

$$
\frac{d f}{d t}=\frac{\Delta P}{2 H G} f
$$

In this chapter, a simple islanding detection based on phase angle difference and over/under frequency is implemented. The islanding detection logic is illustrated in Figure 4.1. If the voltage phase angle difference of the microgrid and utility side is more than 15 degrees, and the absolute value of the microgrid side and nominal frequency difference is more than $0.5 \mathrm{~Hz}$, the circuit breaker is triggered with digital output of the PMU device. 


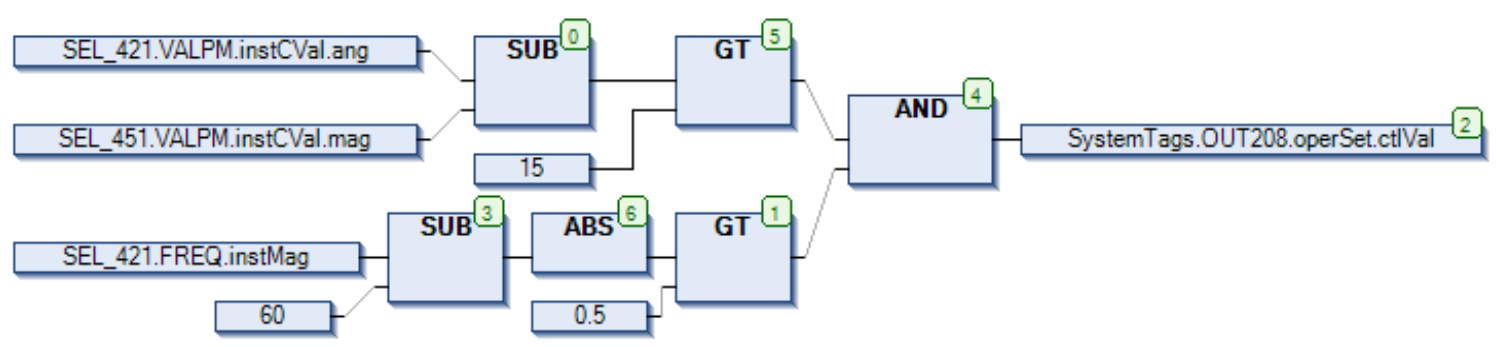

Figure 4.1: Islanding detection function blocks.

Figure 4.2 shows the frequency, voltage and phase angle difference as the response of the PMU circuit breaker upon disconnection of the microgrid from the utility. When the islanding happened at $\mathrm{t}=37^{\text {th }}$ second, the frequency and voltage deviation of the microgrid is shown. However, the measurements from the PMU located on the grid side remains stable.

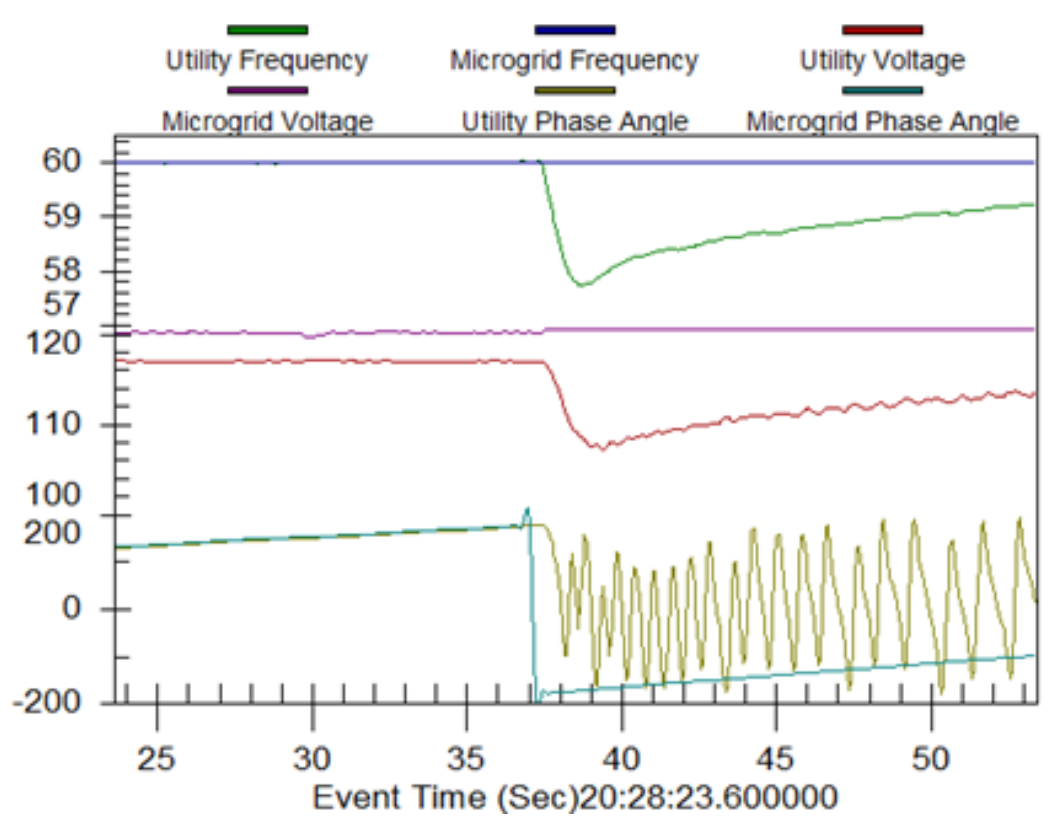

Figure 4.2: Islanding of the microgrid.

An advanced microgrid protection can be performed utilizing PMUs, which allows control by shortening the response time and ensuring protection of the system. Since 
inverter-based DERs do not have inertia, the frequency drop in the figure is the result of the synchronous generator.

\subsubsection{Resynchronization of Microgrids}

A synchrophasor is a viable tool to solve the problem of DER remote resynchronization without a local synchroscope. Following a fault or a disturbance of a microgrid, a part or the complete microgrid might need to be islanded and separated from the remaining network. There are power quality conditions that should be met before resynchronization of the generator with the system. Both sides of the network should have the same frequency, phase sequence and phase angle. Resynchronization of a conventional synchronous generator can be either manual or automatic; and inverter-based DER resynchronizes automatically with the detecting phase angle by a phase-locked loop. The normal limits for generator synchronization are: Slip $\pm 0.067 \mathrm{~Hz}$, voltage 0 - 5 percent and angle \pm 10 degrees.

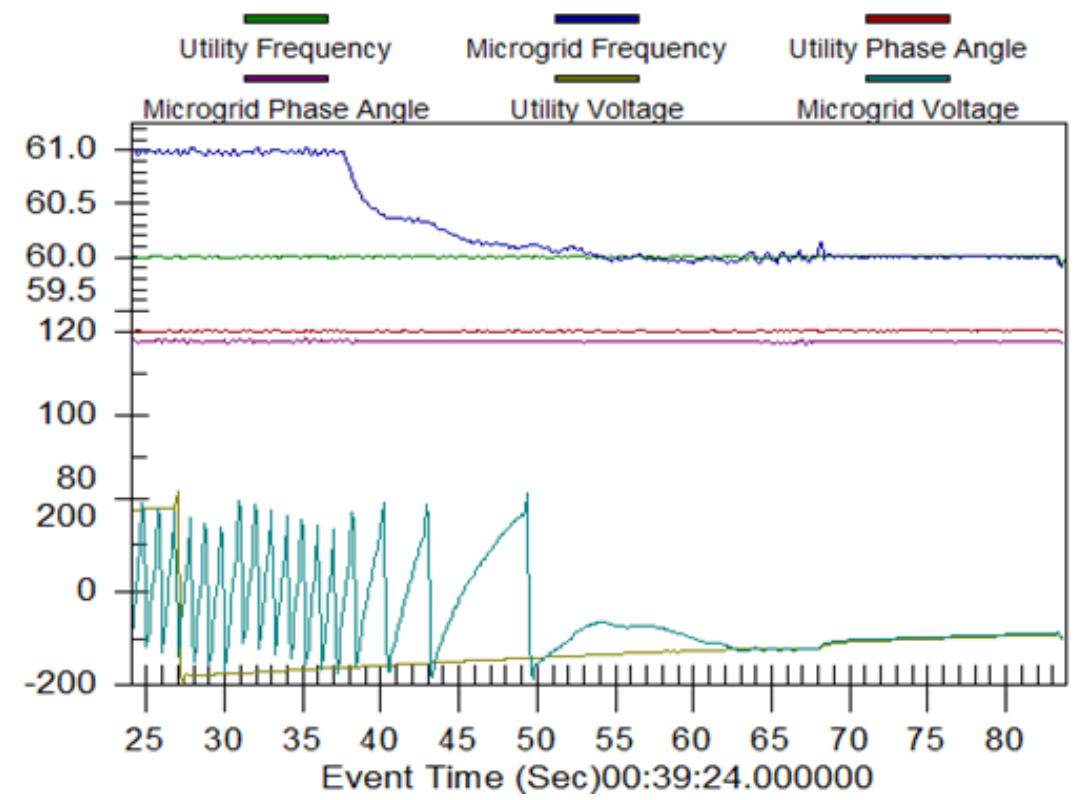

Figure 4.3: (a) Frequency change; (b) Line voltage; (c) Phase angle. 
Figures 4.3. (a), (b) and (c) show the frequency, voltage and phase angle differences of the microgrid and utility side during the remote synchronization process. The figures cover 30 seconds of the synchronization process. In Figure 4.3 (a), from the $55^{\text {th }}$ to $58^{\text {th }}$ second, generator output frequency dropped manually by decreasing the applied torque to generator shaft. At the $58^{\text {th }}$ second, dynamic breaking is enabled to let the synchronous generator follow the utility frequency. At the $70^{\text {th }}$ second, when utility and generator frequency match, the switch is closed. At the $76^{\text {th }}$ second, applied torque to the generator shaft is increased to deliver more power to the system. Figure 4.3 (c) shows phase angle difference between the utility and generator voltage. As synchronization happens at the $70^{\text {th }}$ second, the phase angle difference becomes a value very close to zero. This case shows the ultimate capability of the PMU to monitor and track events in the systems. All events are captured, time stamped and recorded for further investigations and studies.

\subsubsection{Protection and Event Analysis}

Event reports are generated from PMUs to understand the event trigger and to track corrective actions in order to prevent recurrence and provide lessons learned from that particular application. Events should be investigated and analyzed after faults, including any kind of event that causes the problem in the system. This becomes imperative for system security analysis and for revealing the reasons for blackouts or power outages.

In this study, a single phase fault is created to realistically examine the protection scheme with a tripping event. Figure 4.4 shows the dynamic change of voltage and current phasors. Initially, the system is running normally. Once the breaker is closed, phase c is grounded (a backup protection is provided). Over-current protection logic causes tripping of the PMU circuit breaker and flagging a trip event. 

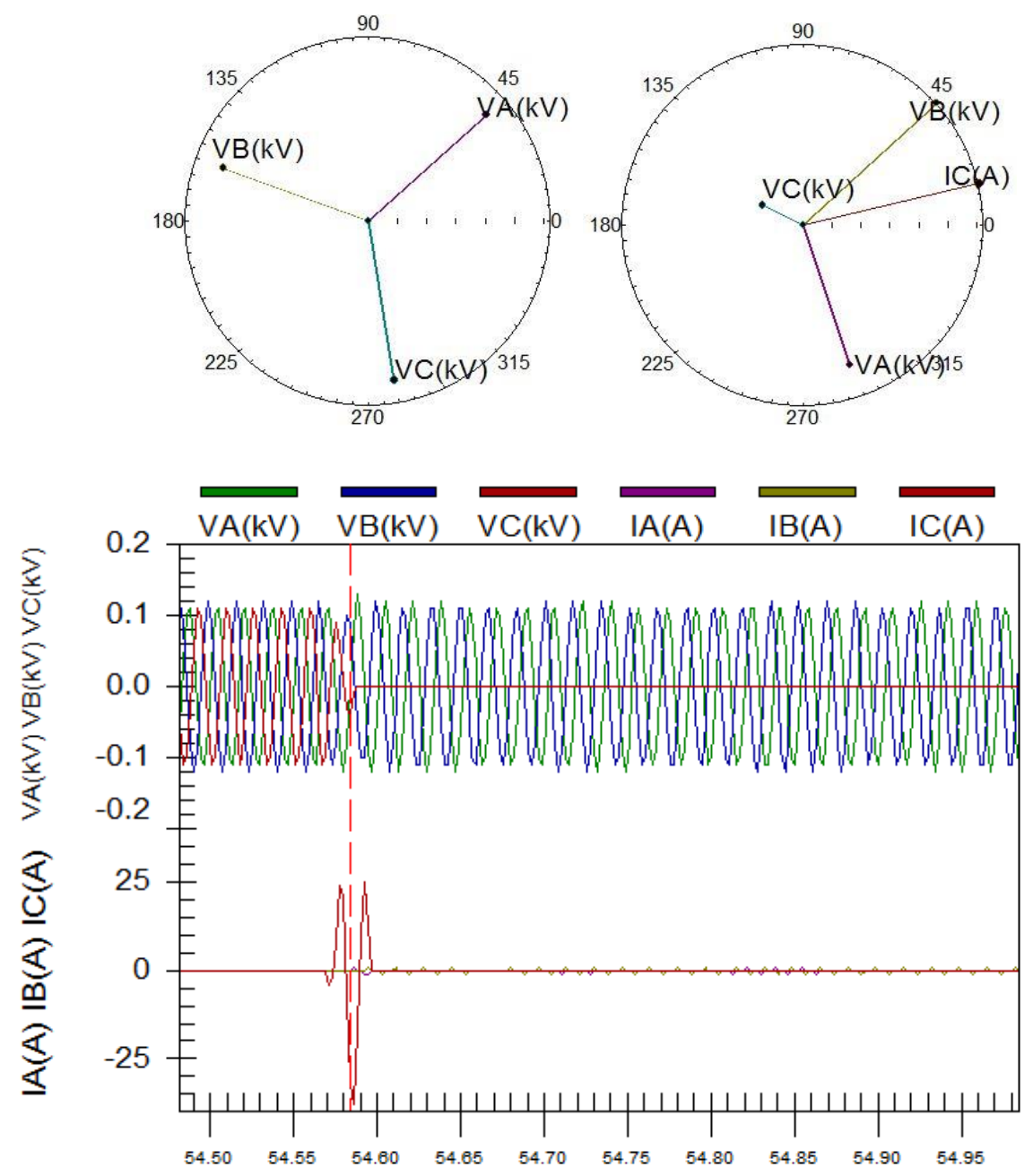

Event Time (Sec)21:30:54.481917

Figure 4.4: Voltage and current phasors; (b) Line voltages (c) Fault current.

Hence, the PMU captured the event and protected the circuit, the backup protection remained ideal. Current on phase $\mathrm{C}$ jumped to $25 \mathrm{~A}$ and voltage on phase $\mathrm{C}$ dropped to zero. Figure 4.4 (a) shows the voltage and current phasors before the fault and during the fault. Figure 4.4 (b) shows the magnitude change of phase voltages. The grounded phase voltage $\mathrm{c}$ becomes zero after the fault. Figure 4.4 (c) shows the short circuit current that appeared during the fault. 


\subsubsection{DER Scheduling and Ancillary Services}

ADNs require complex operations with security constraints, such as voltage limits. DERs can greatly contribute to overcome such problems by scheduling active and reactive power compensation for required feeders. Directly grid-connected renewable DERs can be considered as a negative load source with the intermittent generation profile. This uncertain generation profile can be directly used to inject variable power to the main grid depending on the availability of renewable resources. On the other hand, a battery storage combined DER can be controlled by optimal unit commitment for peak shaving and transmission network congestion management applications. Unit scheduling can be controlled centrally with the proposed architecture. Time-synchronized phasor values can directly provide the phase angle difference between utility grid and microgrid side, which helps to monitor the

power delivered and line loading condition. Active and reactive power flow from the microgrid to network are given in the below equations (4.1) and (4.2). The desired power reference is sent to the embedded controller through PLC, which is controlled via RTAC. RTAC maintains all the measurements from microgrid and acts as the central controller.

$$
\begin{aligned}
& P_{e}=\frac{V_{\text {gen }} V_{\text {grid }}}{X} \sin \delta \\
& Q_{e}=\frac{V_{\text {gen }}{ }^{2}}{X}-\frac{V_{\text {gen }} V_{\text {grid }}}{X} \cos \delta
\end{aligned}
$$

Figure 4.5 (a) shows the change of generated active power from DER. At the $76^{\text {th }}$ second, the injection of active power is increased from $200 \mathrm{~W}$ to $400 \mathrm{~W}$. This power sharing curtails the real power driven from utility. Figure 4.5 (b) shows the resulting current flow from the PCC of the microgrid and utility grid. Figure 4.5 (c) shows the reactive power 
injection in addition to active power. The resulting $1 \mathrm{~V}$ terminal voltage increase is seen from the Figure (4.5).

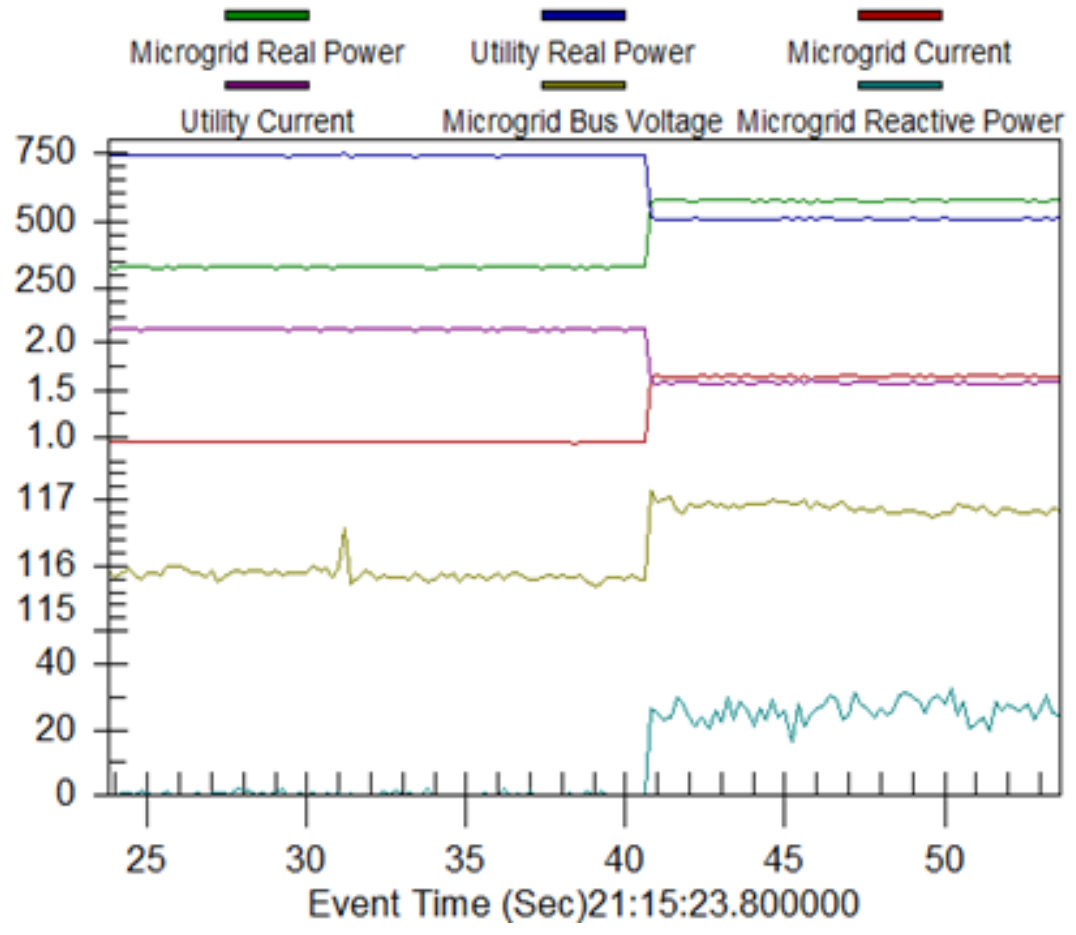

Figure 4.5: Remote power dispatch.

\subsection{Hierarchical Frequency Control}

The objective of this subsection is to present proper centrally coordinated actions to reinstate frequency to its nominal value after a deviation in the system. In a conventional power system operation approach, if an uncontrolled islanding is formed due to emergency conditions, the primary control responds rapidly according to droop adjustments of the generators inside the island. Droop based primary control deviates the frequency from the nominal value, according to the system loading conditions. Upon separation, it may be necessary to shed some of the predetermined loads in the islanded area in order to balance generation and load. 
In Figure 4.6, an islanding situation and hierarchical control scheme is demonstrated. The measurements are taken from synchrophasors deployed on the AC side of the microgrid. During the synchronized operation, the islanded power system is importing power from the remote power system. When the islanding situation takes place at $\mathrm{t}=130 \mathrm{~s}$, the imported power becomes zero.

$$
\Delta P(t)=P_{g e n(t)}-P_{\text {load }(t)}-P_{\text {import }(t)}
$$

As per equation (4.4), the power imbalance inside the microgrid results in a frequency drift in the islanded area:

$$
\Delta P(t)=\left(P_{\text {gen }(t)}-P_{\text {load }(t)}\right)=\frac{2 H_{\text {tot }}}{f_{n}} \frac{d f_{s}}{d t}
$$

The active power imbalance introduces frequency deviation in the islanded microgrid (4.4), where Htot is the total inertia, $f n$ is the nominal frequency, and $f s$ is system frequency. The phase angle difference between the two areas increases. The Figure 4.6 shows the oscillation of the phase angle of the system generators during primary control. Between $\mathrm{t}=130 \mathrm{~s}$ and at $\mathrm{t}=147 \mathrm{~s}$, the primary control is established. The generation in the islanded area increases and the frequency settles in a stable region. At the $t=163 \mathrm{~s}$, the secondary control is initiated to regulate system frequency to the nominal value by power injection from the storage system of the microgrid. At $\mathrm{t}=181 \mathrm{~s}$, the system frequency reaches the minimal value. AGC-based secondary control is used to restore system frequency to the nominal value. A common way to enable AGC in power systems is to implement a PI controller. An area control error (ACE) in a power system is given as equation (4.5), where $B$ is the frequency bias factor, $\Delta P(t)$ is the deviation of active power 
balance in the area, and $\triangle P(A G C)$ is the control command to be sent to the grid-tie inverter. $\beta 1$ and $\beta 2$ are the PI control coefficients.

$$
\begin{gathered}
A C E=\Delta P_{T}+\beta \Delta f \\
\Delta P_{A G C}=-\beta_{1} A C E-\beta_{2} \int A C E d t
\end{gathered}
$$

In real-time applications, angular stability is measured by the difference in generator voltage angles of the two points and compared with a predefined threshold angle during pulsed load:

$$
\Delta \theta_{P M U_{1}}-\Delta \theta_{P M U_{2}} \leq \varepsilon
$$

$\varepsilon$ is defined as the tolerance in degrees. The tolerance in this study is selected to be 10 degrees.

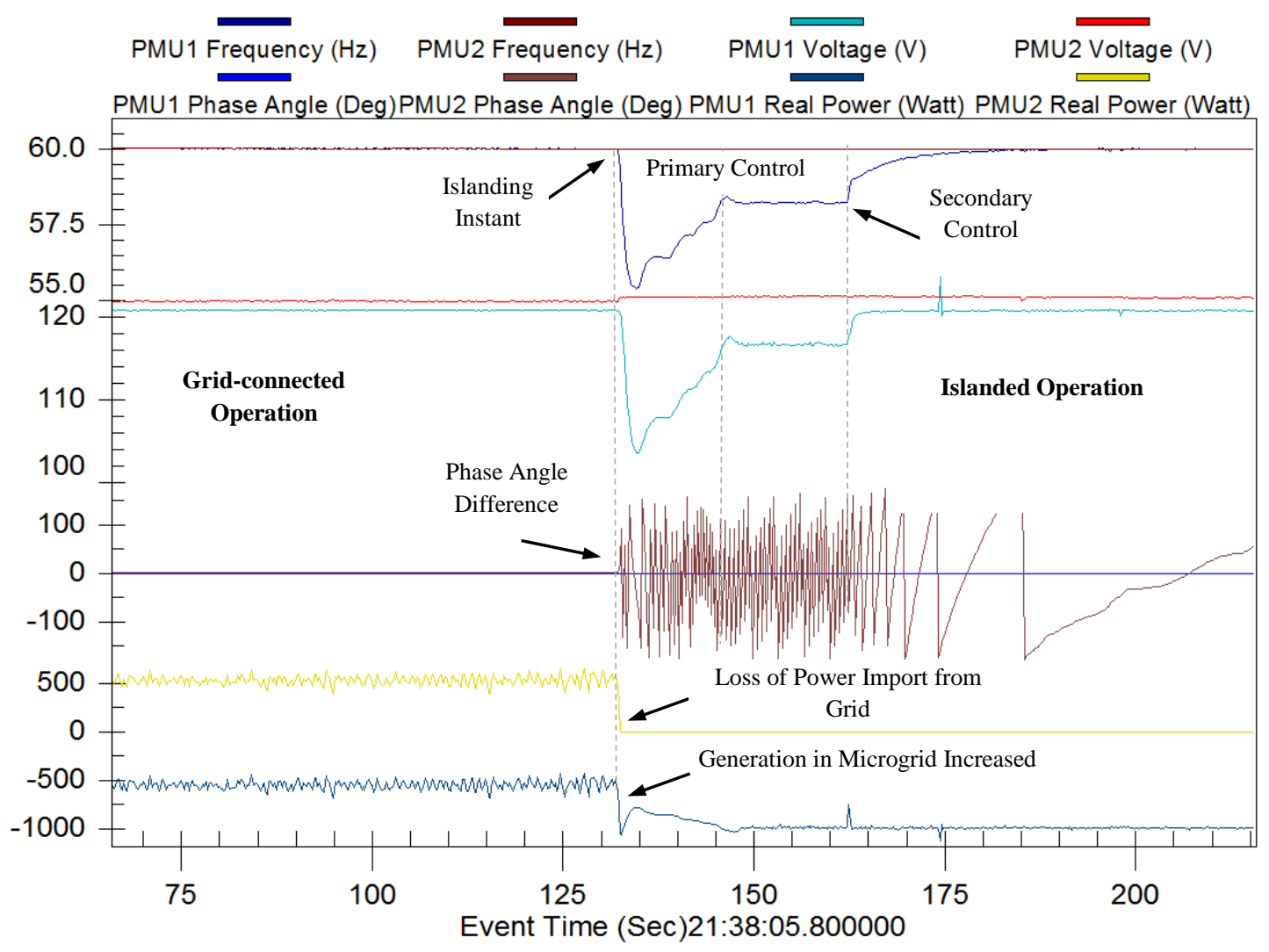

Figure 4.6: Islanding of hybrid AC/DC microgrid. 
When the angular separation between two PMU measurements is exceeded, the instability should check whether the system is in a stable or unstable swing. Specifically, the microgrid buses are assumed as a two-machine system implementing the swing equation to determine the out-of-step condition in the two-area system. As classical generator dynamics are defined as:

$$
\begin{gathered}
\delta_{i}=\omega_{i} \\
H_{i} \frac{d \omega_{i}}{d t} P_{m_{i}}-P_{e_{i}} \\
P_{e_{i}}=E_{i}^{2} Y_{i i} \cos \theta_{i i}+\sum_{\substack{j=1 \\
j \neq 1}}^{n} E_{i} E_{j} Y_{i j} \cos \left(\delta_{i}-\delta_{j}-\theta_{i j}\right)
\end{gathered}
$$

$H i$ is the inertia constant of $i$ th generator, $\delta_{i}$ is the internal voltage angle of $i$ th generator, $\omega_{i}$ is the rotor speed of the $i$ th generator, $P_{e_{i}}$ and $P_{m_{i}}$ are electrical $/$ mechanical output power of the $i$ th generator, $E_{i}$ and $E_{j}$ voltage behind transient reactance, $\mathrm{Y}$ is the admittance matrix reduced at the internal generator node.

\subsection{Performance Monitoring under Pulsed Loads}

To further verify the proposed control algorithm for hybrid AC-DC power system operation with pulse load mitigation, a hardware experiment is demonstrated. PMUs are connected to the synchronous generator and grid-tie inverter buses. System frequency and voltage variations, phase angle displacement and pulsed loading are shown in Figure 4.7.

A $4 \mathrm{~kW}$ pulsed load is applied three consecutive times. It is noticed that the system frequency experiences an oscillation during the transition, which eventually settles to the reference value. The terminal voltage experiences temporary spikes during transition as 
well, and it settled within limits due to reactive power control inside the island. The phase angle displacement between microgrid buses is kept under the 10 degree threshold. From the results, it can be seen that during the pulse load duration, the DC micro grid can inject power to help the system regulate the frequency, which only dropped to around $57.5 \mathrm{~Hz}$. The voltage dropped to around $115 \mathrm{~V}$. The proposed coordinated converter control was able to perfectly mitigate frequency and voltage dips according to pulsed loading. The islanded low inertia hybrid microgrid was able to withstand the pulsed loading conditions.

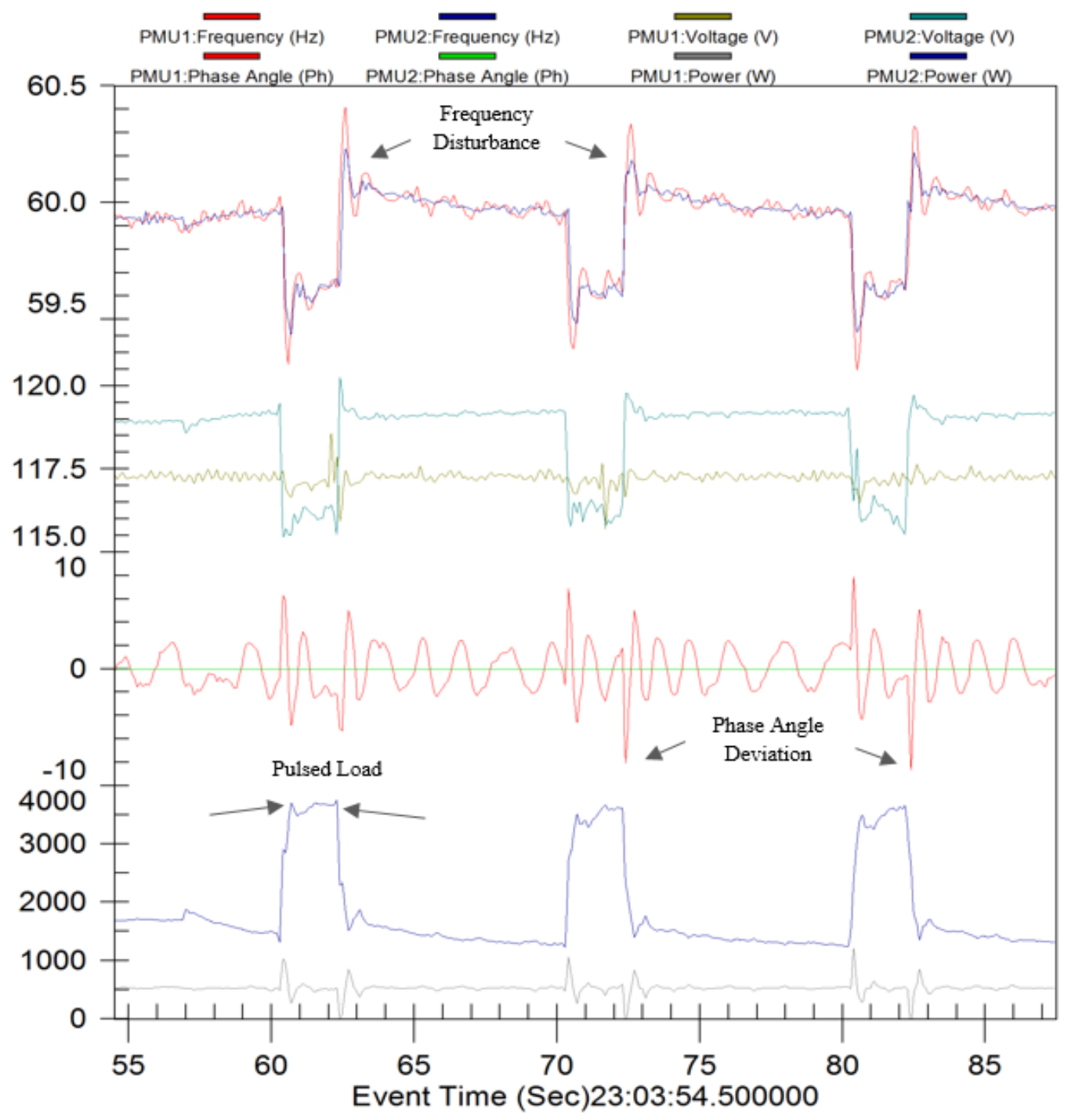

Figure 4.7: Microgrid performance under pulsed loading. 
It is imperative to keep frequency and phase angle displacement in allowable range, since such an impact can potentially cause the microgrid system voltage and frequency collapse momentarily. This impact can be a result of a pulse loading, temporary fault condition or an unintentional switching activity with the microgrid. Using the proposed pulsed-load mitigation, we were able to retain frequency and voltage inside the microgrid within permissible limits. The resiliency of the microgrid was enhanced using power electronic-based inverters, which respond faster to the transients, where synchronous generators fail to respond due to high ramping factor.

\subsection{Summary of the Results and Conclusion}

In this chapter, a synchrophasor-assisted control of hybrid microgrids was investigated. The islanding detection using synchronized measurements was investigated. Remote resynchronization of the DERs, performance monitoring of hierarchical control and pulsed load experiments using time-synchronized measurements was demonstrated. 


\section{PROTECTION OF AUTONOMOUS MICROGRIDS USING AGENT-BASED DISTRIBUTED COMMUNICATION}

\subsection{Introduction}

In this section, we investigate a new scheme for the protection of autonomous microgrids using agent-based distributed communication to overcome overcurrent and frequency protection selectivity problems. A selectivity mechanism is proposed based on feeder characteristics and the location of the IEDs considering the RAS of the microgrid after a fault instant. An online frequency selectivity approach is proposed to avoid pulse load effects in low-inertia autonomous microgrids. The IEC61850 framework and GOOSE protocol messaging are used to enable high-speed peer-to-peer communication capability. To demonstrate the proposed protection scheme, real-time laboratory experiments are performed using the microgrid we have developed.

A microgrid requires implementation of various power system concepts in a smallscale network including stability, control and protection. When a microgrid is not grid-tied and operates in the autonomous mode, this requires certain rules for a stable hybrid system operation, where ac and dc based DERs are linked through power converters [72]. When power converters and conventional electromechanical DERs are harmonized in a microgrid, the detection of the low fault contribution of the inverter-based DERs becomes extremely difficult and ensuring a reliable selectivity becomes challenging.

RAS are designed to remediate the disturbances by changing the network configuration according to predetermined rules, including islanding, generator operation 
mode control, load shedding, and adaptive protection. Considering the enhanced RAS capability of the microgrids, the conventional straightforward downstream-to-upstream delay-based overcurrent protection trip characteristics are becoming inadequate. Since autonomous hybrid ac/dc microgrids are not supported by a strong utility grid, the relatively poor system inertia introduces deteriorating effects to the system stability. Frequency is generally considered as the control reference for the ac side of the grid, where the dc bus voltage value is taken as reference for the dc network. Frequency protection of an autonomous microgrid is also more challenging compared to large transmission and generation networks, specifically in low inertia systems involving a number of power converters. Unlike the normal distribution grid operations, the detection of a heavy loading and fault condition becomes a challenging task. Pulse loads are particular in that they draw high current in a very short time period, which can potentially cause the system voltage and frequency to drop in the entire microgrid momentarily [73]. Pulse loads exist inherently in battery charging platforms, specifically in pulse charging applications. Non-transient disturbances like pulse loads can be confusing for relays to distinguish whether it is a fault or a heavy loading condition.

Thus far, central protection approaches are proposed to calculate the fault current of the DERs in a microgrid according to varying system topologies [74], [75], [76], [77]. The major drawbacks of the centralized methods are the requirement of high communication capability with a powerful central controller, and being susceptible to single-point failures. This vulnerability can easily jeopardize the system with a complete collapse. Most of the central control schemes are not suitable for microgrid operations, since the conventional relay coordination may not be sufficient for frequently varying 
conditions. Therefore, advanced distributed protection schemes and interactive communication are required to overcome microgrid protection limitations.

Due to their inherent resilience, decentralized approaches have drawn considerably more attention than the centralized methods for both protection and RAS. Agent-based decentralized approaches have the ability to self-check, and react accordingly to the prevailing environment conditions. Cooperative agent-based protection schemes are widely discussed in the literature. However, it has never reached a mature level, and stayed in the early development stage [78]-[79]-[80]. Malicious attack-aware agents are utilized to increase the degree of fault tolerance in [81], and an agent-based self-healing reconfiguration scheme has been discussed after a fault instant in [82]. Most of the previous studies in literature do not address realistic circumstances, including overcurrent delay adjustments and frequency protection selectivity.

As DER penetration increases in smart grids, using vendor-independent commonly accepted standardized communications protocols, such as IEC 61850, is becoming crucial [83]. Agent-based protection schemes are becoming feasible to be deployed in realistic applications, including SCADA and fault-event recorders [84]. The emerging international standard IEC 61850 provides an efficient communication and interoperability framework while power system operations are in a transition from a centralized control to some form of decentralized functionality. Based on the logical node definition, IEC 61850 shares similarities to multi-agent systems with horizontal and vertical messaging structures [85]. The integration of IEC 61850 object modeling with executable function blocks enables industrial deployment of multi-agent systems in power systems and automation applications [86]-[87]-[88]. While centralized control schemes rely on a hierarchical long 
line of vertical communication, decentralized control schemes exploit horizontal highspeed peer-to-peer communication. IEC 61850 based generic object oriented substation event (GOOSE) technology enables fast horizontal peer-to-peer communication to exchange messages between two or more devices with decentralized functions in around 0.25 cycle $(4-5 \mathrm{~ms})$ [89].

\subsection{Autonomous Microgrid Setup}

The microgrid and IED setup developed is shown in Figure 5.1. The setup involves a conventional synchronous generator, a grid-tie inverter with a bidirectional power flow capability between ac/dc sides, five lithium-ion battery banks, a PV panel emulator, and ac/dc loads.

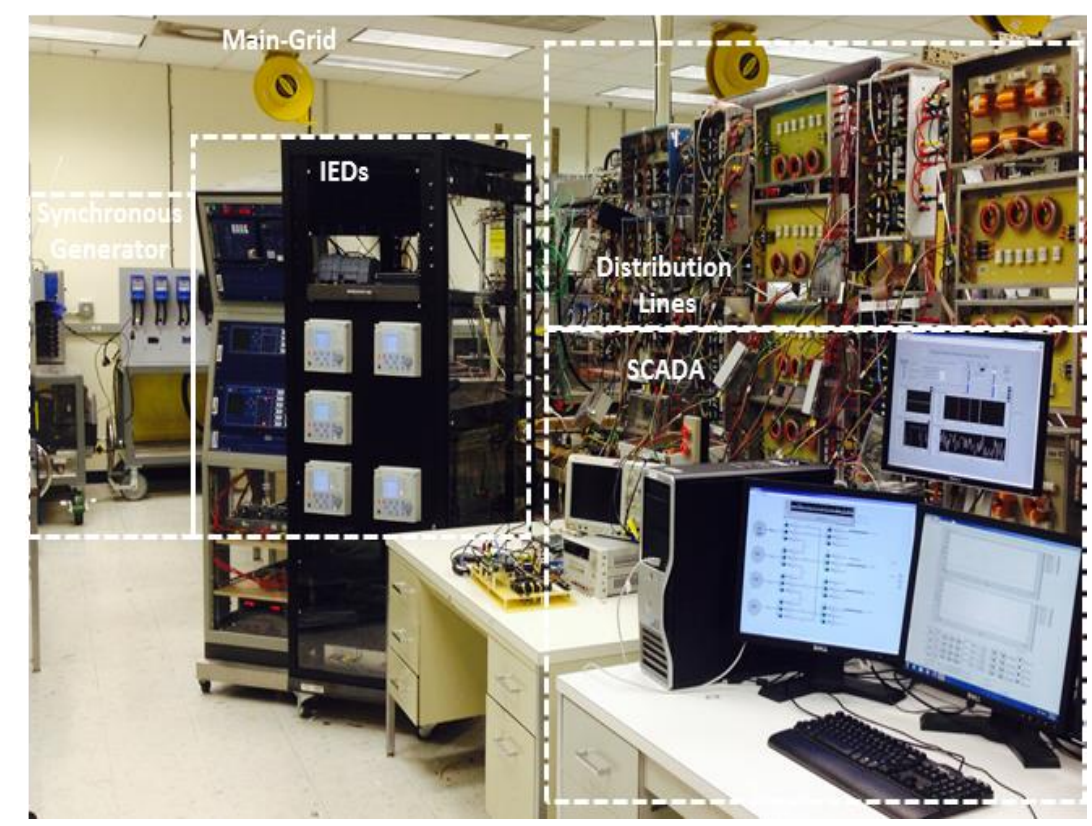

Figure 5.1: Microgrid protection setup.

The IEDs include all required logical nodes, as defined in IEC 61850-7-4 [90]. Two synchrophasor-enabled IEDs are employed in microgrid main buses. The available data of 
the DC microgrid is mapped to OPC UA middleware as the representation of IEC 618507-420 [91] data models to ensure interoperability with all logical nodes [92], [93]. The microgrid infrastructure, IED deployment on microgrid feeders and the related logical nodes are shown in Figure 5.2.

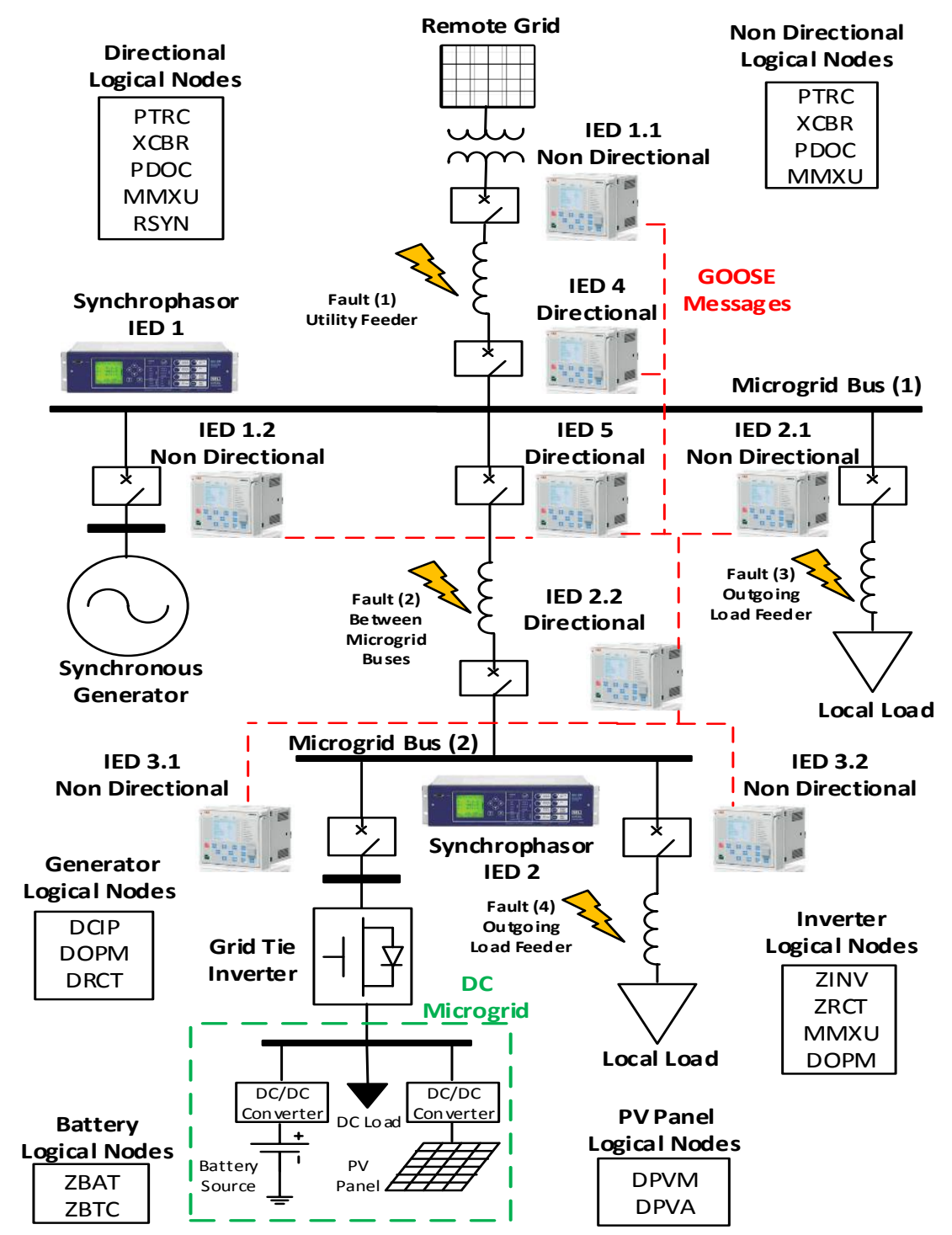

Figure 5.2: IED deployment on microgrid feeders and the related logical nodes 
In this study, all IEDs are located on the same local area network (LAN), however a special router configuration can be applied for wide area network (WAN) GOOSE messaging [94]-[95].

\subsection{Overcurrent Protection Selectivity}

This subsection introduces overcurrent selectivity challenges, the agent-based proposed algorithm and real-time experimental results.

\subsubsection{Overcurrent Protection Selectivity Challenges}

Conventional power system protection requires the protective devices to be connected in series, and the trip characteristics of the downstream protective device must be faster than the upstream devices. However, in hybrid ac/dc microgrids, the following selectivity problems arise:

- The inverter-based DER's fault contribution is limited to between $100 \%$ and $200 \%$ of the nominal operational current according to the implemented current control scheme [96]. When inverter-based DERs are harmonized with electromechanical generators, the detection of the low-fault contribution of inverter-based DER becomes extremely difficult and ensuring reliable selectivity becomes challenging.

- The current practice of islanding detection requires islanded areas to cease from being energized within 2 seconds. This requirement drastically reduces the reliability and resiliency of the microgrid operation [96]. However, microgrids are expected to continue operation with a proper RAS capability and efficient supply reserves after unintentional islanding following a fault clearance. RAS schemes, including seamless 
islanding, generation operation mode control and load shedding practices, eliminate the mandatory shutdown requirement, thus increasing the reliability and resiliency.

Figure 5.3 illustrates the possible fault current routes in a microgrid structure featuring a synchronous generator, an inverter DER, and a grid connection point. The double arrows define the non-directional functionality, where the faults can be detected in both directions. In such a microgrid topology, a variety of network topologies would prevail depending on the fault location resulting an islanding condition, a simple isolation of the load feeders or a complete shutdown of the system.

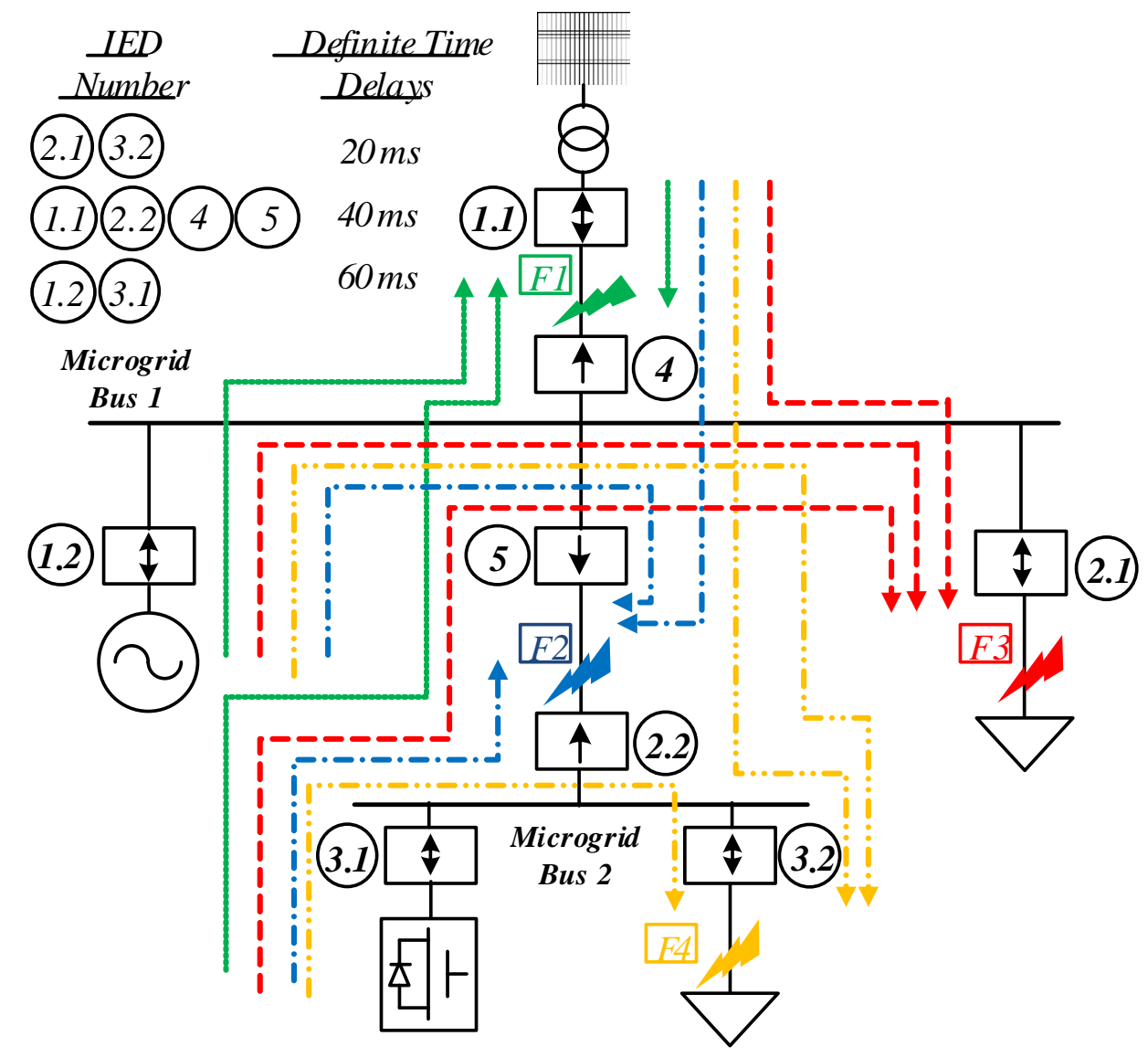

Figure 5.3: Fault current routes for different fault points. 
In normal operating conditions, the fault contribution of the different DERs have the below order:

$$
I f_{\text {Grid }} \geq I f_{\text {Generator }} I f_{\text {Inverter }}
$$

Table I shows different fault current levels seen by IEDs. An IED can detect different fault contributions depending on the location and feeder type.

Table 5.1: Fault currents for grid-connected operation

\begin{tabular}{|c|c|c|c|c|c|}
\hline IED & Direction & F1 & F2 & F3 & F4 \\
\hline \hline IED 1.1 & None & If grid & If grid & If grid & If grid \\
IED 4 & Reverse & If gen+inv & - & - & - \\
IED 5 & Forward & - & If grid+gen & - & If grid+gen \\
IED 1.2 & None & If gen & If gen & If gen & If gen \\
IED 2.1 & None & 0 & 0 & If grid+gen+inv & 0 \\
IED 2.2 & Reverse & If inv & If inv & If inv & - \\
IED 3.1 & None & If inv & If inv & If inv & If inv \\
IED 3.2 & None & 0 & 0 & 0 & If grid +gen+inv \\
\hline
\end{tabular}

** If grid, If inv, If gen are the fault contributions of the main-grid, inverter-based DER and synchronous generator, respectively.

A fault current can be fed from various directions and DERs depending on the fault location. For such a network topology, it is impossible to define a conventional delay-based protection scheme. It is crucial to establish an agent-based distributed communication allowing the IEDs to communicate with each other to decide which circuit breakers should clear the fault. In this configuration, IED4, IED5 and IED2.2 are selected as directional relays. IED4 and IED2.2 are set to pick-up only in the reverse direction, where the current flow is upstream. IED5 is set to pick-up only in the forward direction, where current flow is downstream. For islanded operations, the main grid will no longer contribute to the fault 
current. IED5 and IED2.2 can detect very low or very high fault currents for different fault points.

\subsubsection{Agent-based Overcurrent Selectivity Method}

Unlike the conventional consecutively increasing time delay based protection schemes, the proposed approach aims to define tripping priorities according to the upstream/downstream connection and the location type of the IEDs considering the RAS scheme. Three definite time delay (DT) levels are adopted: 1) simple load feeder; 2) bus routing; 3) DER feeder.

- Simple load feeder IEDs: Their tripping does not risk the entire microgrid operation. These relays only detect the faults appearing downstream of the IEDs, which eases the selectivity. Thus, these IEDs are set to operate as quickly as possible under a fault condition with the shortest delay time. A time delay of $20 \mathrm{~ms}$ is chosen.

- Bus routing feeder IEDs: They are responsible for managing the directional power flow inside the microgrid. The islanding and RAS are handled by these IEDs. To ensure a reliable selectivity, a delay time in between simple load feeder IEDs and DER feeder IEDs must be chosen. In this scheme, a time delay of $40 \mathrm{~ms}$ is chosen.

- DERfeeder IEDs: The RAS is possible as long as the DERs continue operation during the fault clearance period. A successful RAS enables generation/demand balance to keep system frequency and voltage in permissible limits. Thus, the maximum delay time must be defined for these IEDs protecting DERs in the microgrid. A time delay of $60 \mathrm{~ms}$ is chosen. 
Table 5.2 shows the defined time delays of the microgrid with respect to IED locations.

Table 5.1: Fault currents for grid-connected operation

\begin{tabular}{|c|c|c|}
\hline \hline IED Name & IED Location Type & Definite Time Delay \\
\hline IED1.1 & Bus Routing & $40 \mathrm{~ms} \approx(2$ cycles $)$ \\
\hline IED1.2 & DER feeder & $60 \mathrm{~ms} \approx(3$ cycles $)$ \\
\hline IED2.1 & Load feeder & $20 \mathrm{~ms} \approx(1$ cycle $)$ \\
\hline IED2.2 & Bus Routing & $40 \mathrm{~ms} \approx(2$ cycles $)$ \\
\hline IED3.1 & DER feeder & $60 \mathrm{~ms} \approx(3$ cycles $)$ \\
\hline IED3.2 & Load feeder & $20 \mathrm{~ms} \approx(1$ cycle $)$ \\
\hline IED4 & Bus Routing & $40 \mathrm{~ms} \approx(2$ cycles $)$ \\
\hline IED 5 & Bus Routing & $40 \mathrm{~ms} \approx(2$ cycles $)$ \\
\hline
\end{tabular}

DT levels are in below order:

$$
D T_{D E R}>D T_{D E R}>D T_{L o a d}
$$

Overcurrent pick-up settings are determined through an offline protection analysis, according to Table 5.1, and embedded in active settings of the IEDs. Figure 5.4 shows the overall GOOSE peer-to-peer message traffic between IEDs for fault cases F1, F2, microgrid bus 1 (MG1) and microgrid bus 2 (MG2), including interlocking signals to block tripping and other control operators, including the DER mode of operation change and active setting control. 


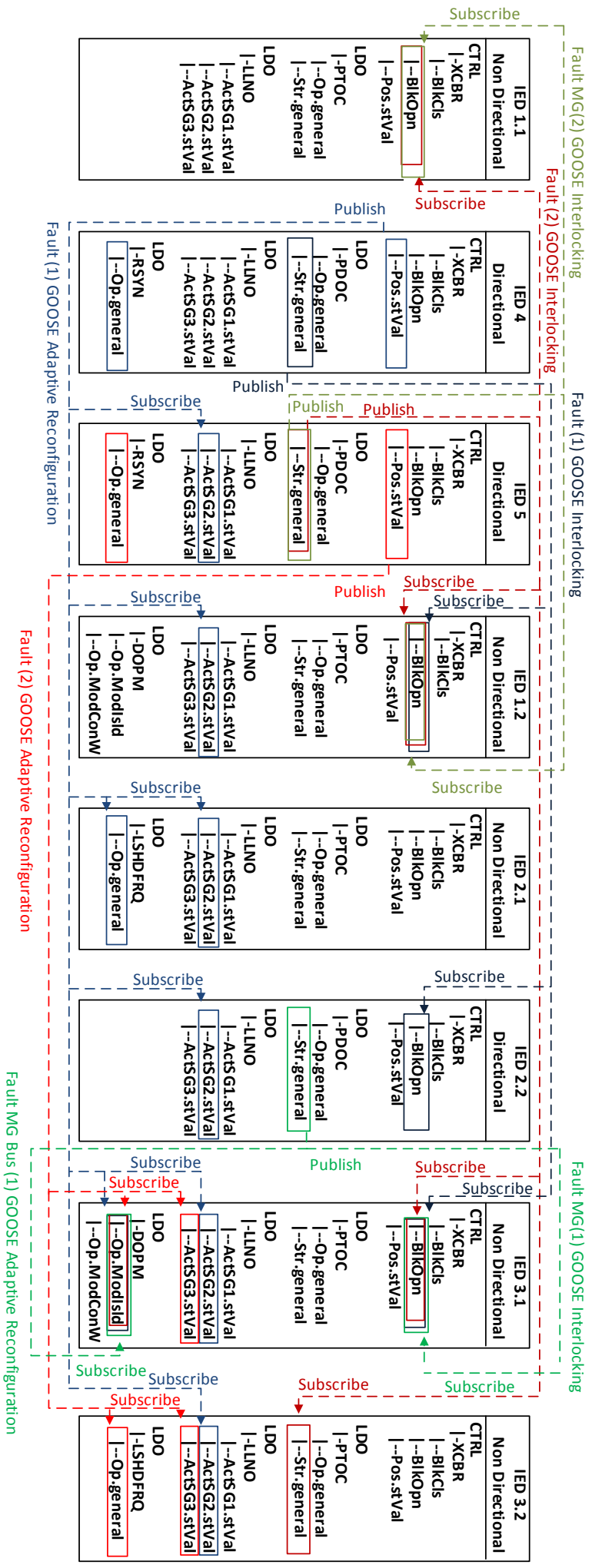

Figure 5.4: Publisher / subscriber pattern and GOOSE message traffic. 


\subsubsection{Case I: Fault at Utility Feeder}

At this fault point, IED1.1, IED4, IED1.2 IED2.2 and IED3.1 detect the fault current. IED4 detects the overcurrent on the reverse direction, which occurs only when the fault current is headed to upstream in the microgrid. Thus, IED4 can be selected as the main decision unit for the fault clearance in this fault point. A satisfactory clearance can be accomplished by tripping only IED1.1 and IED4. Accordingly, the microgrid can continue operation in islanded form once the fault is cleared.

When the directional protection function of the IED4 detects reverse overcurrent, the pick-up "Start" operator becomes high, the internal timer is initiated, and a blocking signal is published to IED1.2, IED2.2 and IED3.1 in order to block the opening of circuit breakers. Since IED1.1 and IED4 have the same definite time delays, the tripping is accomplished with an "Operate" operator when their timers expire after the 40 ms delay. Following the fault clearance, the microgrid transitions to autonomous islanded mode. Depending on the former import/export power exchange between the microgrid and the main grid, the resulting power imbalance would create a deviation in the system voltage and frequency beyond permissible limits. A system collapse is unavoidable unless an adaptive reconfiguration is assured with proper power sharing control, and a successful load shedding in the microgrid.

DER feeder IEDs (IED1.2 and IED3.1) would provide generation and load balance by adjusting the system frequency and voltage to permissible limits. A load shedding scheme can be enabled by load feeder IEDs (IED2.1 and IED3.2). According to the prevailing network topology, a new operation setting is enabled for all IEDs operating in the autonomous mode. The microgrid would resynchronize with the grid once the fault is 
cleared. This function is enabled by the synchronism check (RSYN) logical node of the IED4. The logical node considers microgrid frequency, phase angle difference and voltage amplitude with the main grid.

\subsubsection{Case II: Fault at Bus Connection Line}

At this fault point, IED1.1, IED1.2, IED5, IED2.2 and IED3.1 detect the fault current. A satisfactory clearance can be accomplished by tripping only IED5 and IED2.2. Accordingly, the microgrid would split into two parts, where the upper part remains gridconnected, and the lower part can continue operation in the islanded mode. For this fault point, IED5 and IED2.2 are selected as directional relays, where IED5 detects only the downstream overcurrent, and IED2.2 detects overcurrent headed to upstream. Therefore, an advanced selectivity can be organized with IED5 and IED2.2. IED5 continuously checks the IED2.2 pick-up "Start” bit through the GOOSE subscriber. For any fault in the system, when both IED5 and IED2.2 detects an overcurrent, then the fault is determined as between IED5 and IED2.2. IED5 coordinates the fault clearance process accordingly, when the IED5 "Start" operator is high, and the IED3.2 "Start" operator is low. Then, IED5 publishes the blocking signals to IED1.1, IED1.2 and IED3.1. The new operation active settings are established.

\subsubsection{Fault on Load Feeder}

At this fault point, IED2.1, IED1.1, IED1.2, IED2.2 and IED3.1 detect the fault current. The desired operation is only tripping IED2.1. Accordingly, the microgrid can continue operation without any reconfiguration. IED2.1 detects the faults only to downstream, which makes the selectivity simple and straightforward. In this case, no 
operation mode and setting change are necessary. Fault F4 protection requires almost the same selectivity method as F3.

\subsubsection{Fault at Microgrid Bus}

At this fault point, IED1.1, IED1.2, IED2.2 and IED3.1 detect the fault current. The directional bus routing IED2.2 coordinates the clearing process by continuously checking the upper-level bus routing IED5 and IED4 pick-up operators. Even though IED4 does not detect the fault, the low start operator crosscheck is mandatory to differentiate the MG1 from F1. When IED2.2 "Start” operator is high, but IED5 and IED4 "Start” operators are low, the fault is determined to be on microgrid bus1. IED2.2 publishes the blocking signal to only IED3.1, as well as the mode of operation change command from the constant active power (W) to voltage frequency regulation (V-f) mode. To establish a further RAS, a loadshedding signal can be published to IED3.2. Since the fault is sustained on the microgrid bus, IED1.1 and IED1.2 trip after the 40ms and 60ms delay time, respectively.

In this fault point, the satisfactory clearing process should be the isolation of microgrid bus 2 and all connected IEDs. Thus, DERs and loads connected to microgrid bus 1 can continue grid-connected operation. At this fault point, IED1.1, IED1.2, IED5 and IED3.1 detect the fault current. IED5 coordinates the fault clearing process accordingly, when IED5 "Start” operator is high, and IED2.2 "Start” operator is low. Although it is not essential, a crosscheck mechanism can be applied by the simple load feeder IED3.2 start operator's low value to differentiate the MG(2) from F4. IED5 publishes the blocking signals to IED1.1 and IED1.2. Since the fault is sustained on the microgrid bus, IED3.1 trips after a $60 \mathrm{~ms}$ delay time. Since microgrid bus 1 continues to operate in grid-connected mode, no mode of operation switching mechanism is required. 


\subsubsection{Real-Time Implementation of Agent-based Overcurrent Protection}

To validate the proposed protection scheme, F1 fault scenario was realistically demonstrated. The fault was created by applying an $18 \mathrm{Ohm}$ high fault impedance to the F1 fault point on the distribution line between IED1.1 and IED4. Figure 5.5 shows the fault current contribution of the synchronous generator, inverter-based DER and the main grid during the fault instant.

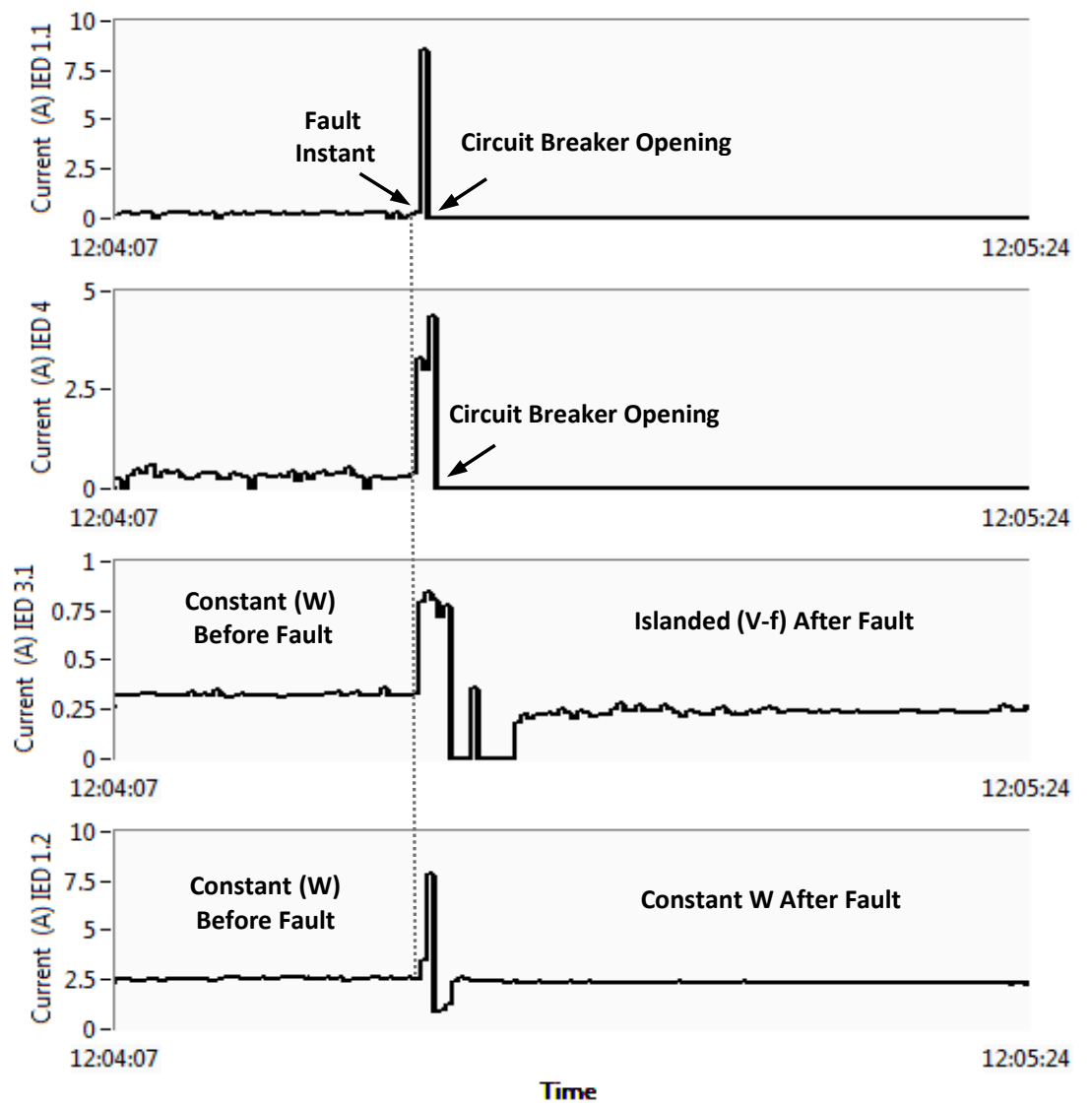

Figure 5.5: Fault currents for F1.

Initially, the microgrid was exporting power to the main grid. The non-directional IED1.1 detects the overcurrent in the forward direction, and the directional IED4 detects the overcurrent in the reverse direction. IED3.1 and IED1.2 also detect the fault overcurrent. 
Figure 5.6 shows the pickup moment of the four IEDs detecting the fault overcurrent.
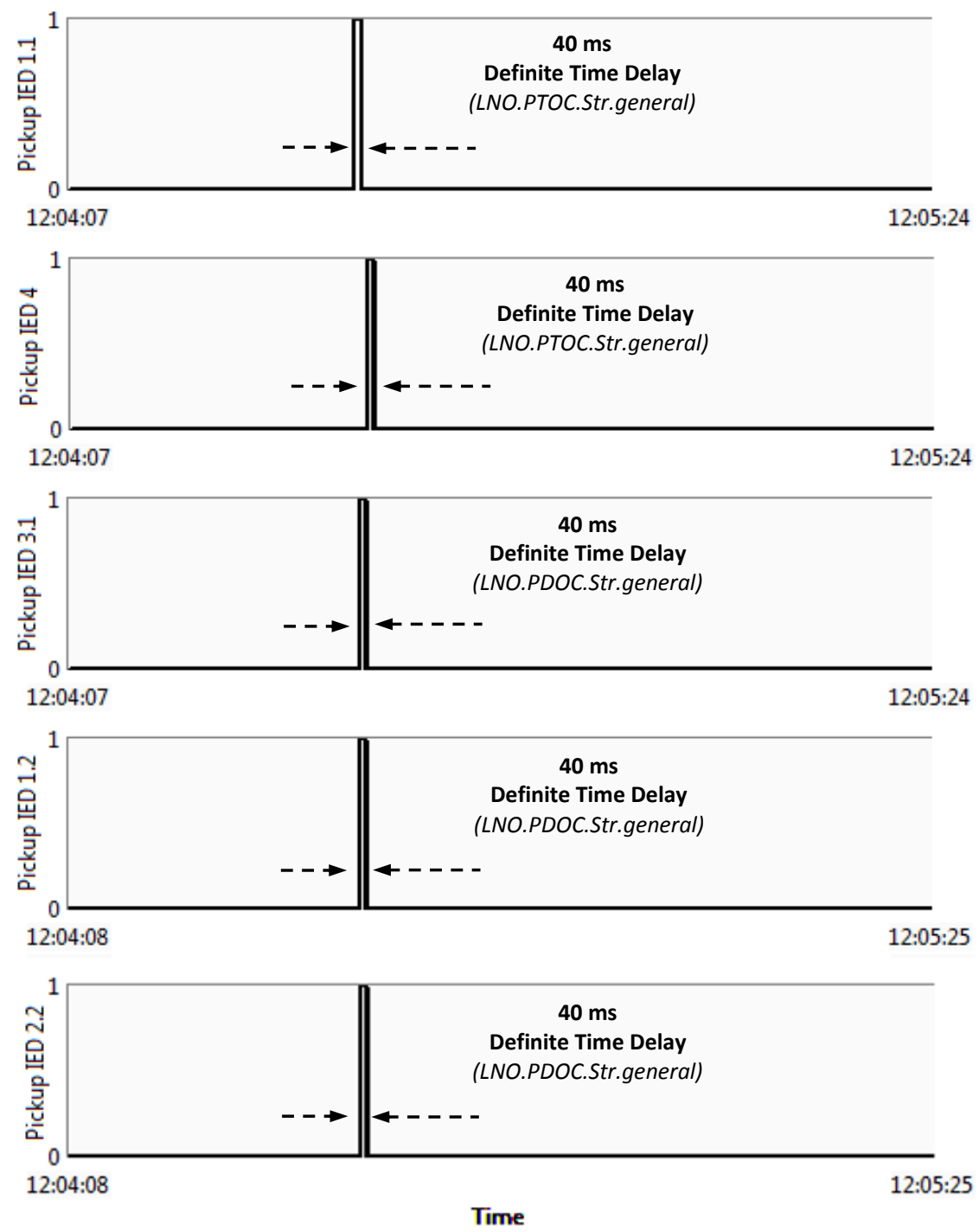

Figure 5.6: Pick-up of the protection function.

As shown in Figure 5.6, during the fault instant, IED1.1, IED4, IED3.1 and IED1.2 pick-up operators become high. However, the desired operation requires only IED1.1 and IED4 to clear the fault. The pick-up duration is $40 \mathrm{~ms}$ for both IED1.1 and IED4. Since IED3.1 and IED1.2 are DER feeder IEDs, their pick-up duration was set to $60 \mathrm{~ms}$. 
While the IED4 pick-up operator was high, the corresponding GOOSE messages were published on the network to block IED1.2, IED3.1 and IED2.2 circuit breakers, as in Figure 5.7.

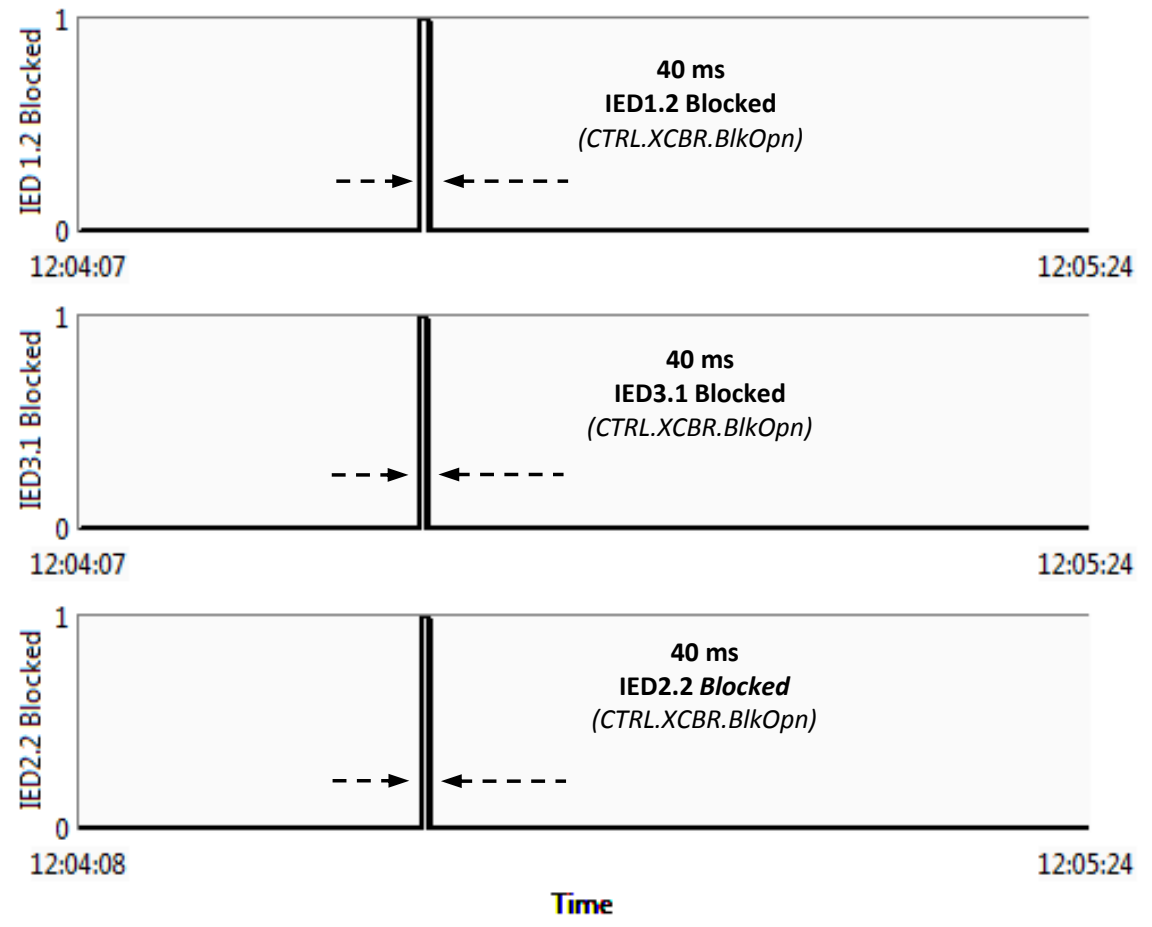

Figure 5.7: Blocked circuit breaker during IED4 pick-up.

When the GOOSE messages were received by the IEDs, the CTRL.XCBR.BlkOpn operator became high, blocking the execution of the circuit breaker opening command. IED1.1 and IED4 cleared the fault by opening the circuit breakers when the pick-up timer was expired and the opening operation was executed. Figure 5.8 shows the circuit breaker position status and active settings of the IEDs. IED1.1 and IED4 circuit breakers are initiated to open once the LDO.PTOC.Op.general operators become high. 


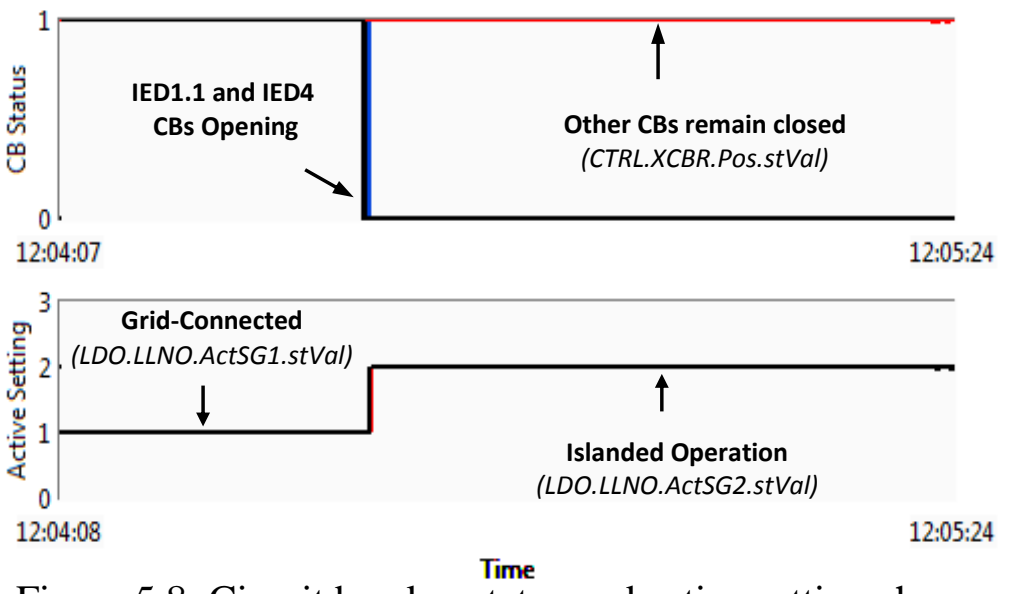

Figure 5.8: Circuit breaker status and active setting change.

As shown in Figure 5.8, only IED1.1 and IED4 trip, and the other circuit breakers remain closed for the islanded operation. The active setting of the IEDs was altered from LDO.LLNO.ActSG1.stVal to LDO.LLNO.ActSG2.stVal. For this specific example, three setting groups were defined with respect to Table 5.1. ActSG1 defines the settings in gridconnected operation mode. ActSG2 defines the settings after F1, and ActSG3 defines the settings after F2. Initially, all IEDs were at setting group-1 ActSG1. Only one active setting can be valid in run time. As shown in Figure 5.8, all IEDs were switched to ActSG2.

After the fault-clearing process, when the IED4 circuit breaker status CTRL.XCBR.Pos.stVal became zero, the microgrid transitioned from grid-connected operation mode to islanded mode. In order to adapt IED settings according to prevailing network topology, a GOOSE message was published to switch operation mode of the IED3.1 controlled inverter-based DER from fixed active power injection mode “LNO.DOPM.Op.ModConW" to "LNO.DPOM.Op.MdIsld" voltage-frequency regulation mode. Figure 5.9 shows the fault clearance, islanding instant and frequency recovery by inverter-based DER following to operation mode change from constant (W) to voltage frequency regulation (V-f) mode. 


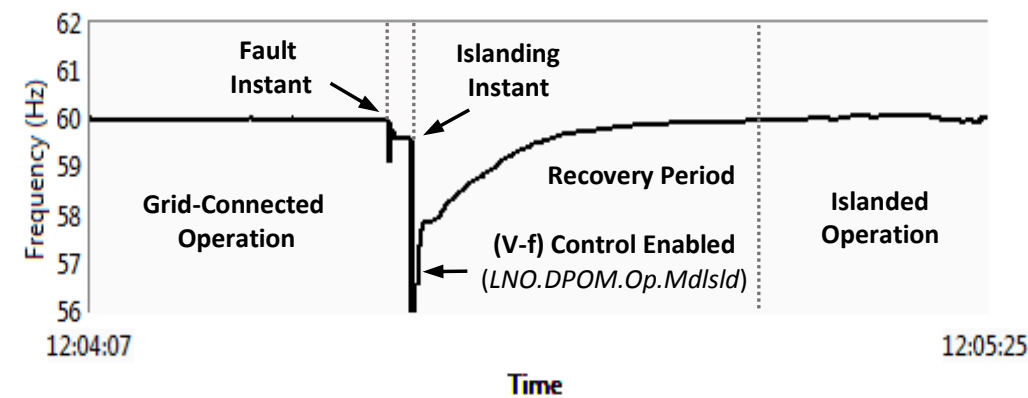

Figure 5.9: Microgrid Frequency recovery after islanding.

Figure 5.10 shows the voltage variation along with active and reactive power before, during and after the fault instant.
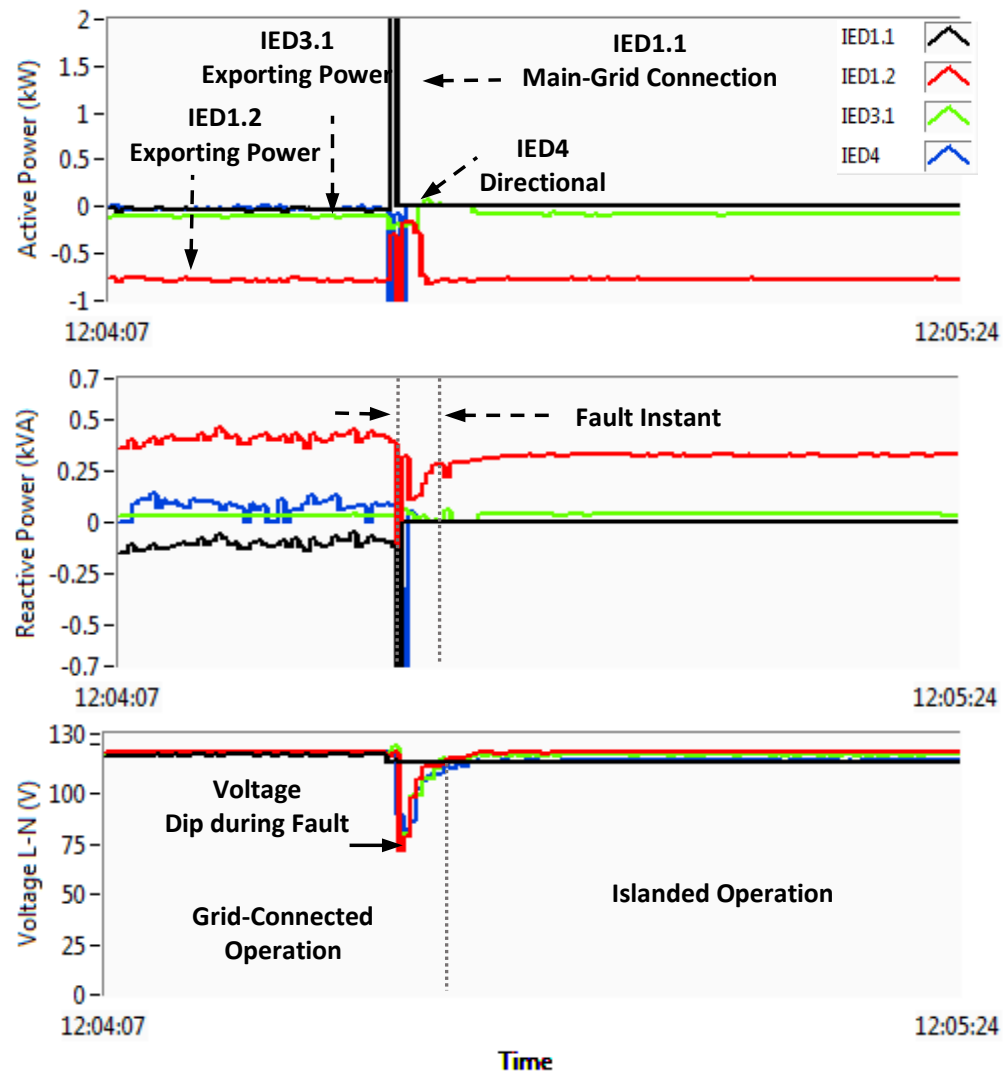

Figure 5.10: Voltage, active and reactive power change during islanding.

Initially, the synchronous generator and inverter-based DER were supplying almost all the demand in the microgrid. The power exchange between the microgrid and main- 
grid was very low. At the fault instant, the system experienced high spikes on active and reactive power, and a voltage dip. Once the fault was cleared, the system voltage settled down to the nominal value for the islanded operation.

\subsection{Frequency Protection Selectivity}

This subsection introduces frequency protection selectivity challenges, the agentbased proposed algorithm and real-time experimental results.

\subsubsection{Frequency Protection Selectivity Challenges}

After a microgrid transitions to the autonomous mode, the frequency support is no longer available from the main grid. The low inertia microgrid should be able to continue operation with scarce generation resources. Depending on the microgrid inertia, pulse loads can deviate the system frequency and voltage, surpassing the maximum permissible limits. For autonomous microgrids, the allowable frequency band $(59.3$ to $60.5 \mathrm{~Hz})$ is wider than wide-area power system networks.

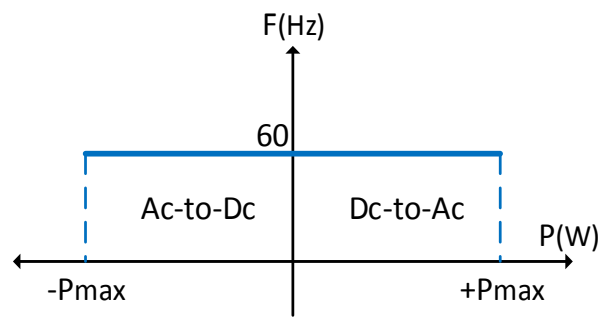

(a) Bidirectional converter is ochronous operation

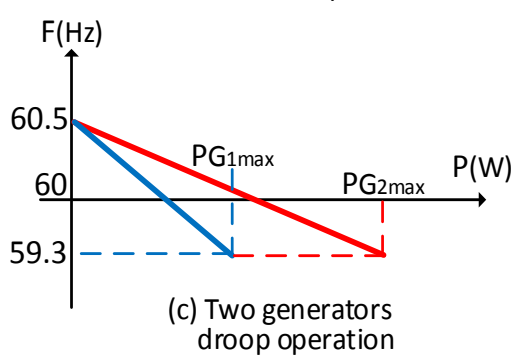

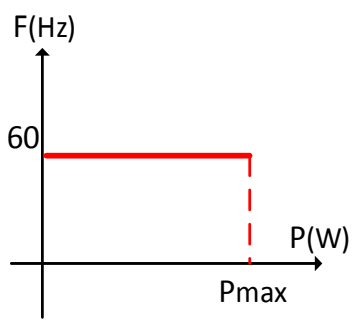

(b) Generator isochronous operation

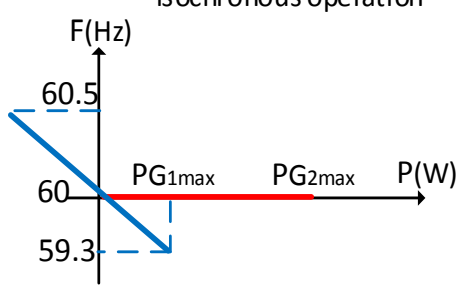

(d) Combined isochronous droop operation

Figure 5.11: Operation modes of autonomous microgrids. 
Over/under frequency, ROCOF and vector surge relays (VSR) are commonly used for conventional frequency protection schemes with predefined thresholds. However, in an autonomous microgrid, varying inertia and pulse loads introduce selectivity complexity in application. Various network topologies would prevail depending on the connected DER types. Inverter-based DER-dominated microgrids are more prone to instant frequency and voltage dips under pulse-loading conditions than synchronous DER dominated microgrids due to their low-inertia. Therefore, conventional frequency protection schemes must be reevaluated considering the various operating conditions of a microgrid by online adaptation and distributed agent-based communication.

Recalling Figure 5.9, during the fault instant and supply/demand imbalance moment, the frequency drops beyond permissible limits. In pulse load applications, a similar frequency impact can be observed. Such impact can potentially cause the system voltage and frequency to drop in the entire microgrid, momentarily. Using the proposed overcurrent protection scheme, we were able to retain complete protection inside the microgrid at most a $60 \mathrm{~ms}$ wait time period. However, this high-speed overcurrent selectivity introduces an additional frequency selectivity problem by lowering the microgrid immunity to the transient pulse load disturbances. The frequency protection functions can pick up and operate unexpectedly. A distributed agent-based protection scheme can be utilized to enable blocking conditions while pulse loads are on duty. In this way, pulse load and faults can be differentiated using a distributed communication.

The frequency control method of an autonomous microgrid drastically affects the frequency and voltage response of the system, and accordingly the protection scheme. Isochronous and droop controls are two fundamental frequency control concepts for typical 
autonomous microgrid operations. It is imperative to consider the operation modes of DERs before designing a proper frequency protection scheme. Power electronic-based inverters respond more quickly to the load changes, where they pick up the changes initially until the synchronous generator reaches the steady state load sharing adjusted by droop setting. This situation also creates improper transient power sharing, which may influence the protection scheme.

- Isochronous Operation Mode: A single, considerably large generation unit is selected to accomplish no final speed change as load increases or decreases. In small autonomous microgrids, this method would provide an efficient way to respond to load changes. However, it is not feasible to employ such a method, where large number of generation units exist, since a proper power sharing cannot be provided. In a hybrid microgrid system, an isochronous synchronous generator can cover residual power imbalance on the ac side only. However, a grid-tie inverter has a capability to transfer power in a bidirectional manner, either from the ac side to dc side, or the dc side to ac side. Figure 5.11 (a) shows the bidirectional inverter isochronous operation mode, where the measured power flow in the ac grid connection point can be negative or positive. Figure 5.11 (b) shows the single direction operation of the synchronous generator, which can only export power.

- Droop Operation Mode: The power sharing is enabled by local DER controllers with respect to frequency change in an autonomous microgrid. As illustrated in Figure 5.11 (c), the frequency is varying due to load changes. This situation introduces high complexity in frequency protection designs. 
Combined Droop and Isochronous: This method can be implemented to mitigate the pulse load effects, where power sharing is facilitated until the transient response of the isochronous unit reaches its normal operation limit. Operation of two units is shown in Figure $5.11(\mathrm{~d})$.

\subsubsection{Agent-based Frequency Protection Method}

A distributed agent-based protection scheme is proposed based on online inertia calculation and an adaptive blocking while pulse loads are on duty. We presume each pulse load as an agent determining its possible impacts to the system, and enables blocking scheme accordingly. The inertia constant is continuously varying due to the number of connected power converters, hence it should be monitored online. Accordingly, the frequency protection references must be adopted online due to the uncertainty in system inertia. The frequency variation of a microgrid is given under loading conditions, where the momentary variation is mainly dependent on the moment of inertia constant:

$$
\Delta P_{\text {dif }}(t)=\Delta P_{\text {dif }}(t)-\Delta P_{\text {load }}(t)=2 H_{\text {tot }} \frac{d \Delta f(t)}{d t}+D \Delta f(t)
$$

where $\triangle P m e c$ is the mechanical power, $\triangle P$ Pload is the electrical load demand, $\triangle P$ dif is the instant load-generation imbalance, Htot is the total microgrid inertia constant, $D$ is the load damping, and $\Delta f$ is the system frequency.

Online inertia estimation can be calculated with synchrophasor IEDs by introducing a small load perturbation for a sample period. Online inertia is calculated with a known sample period, active power change and two consecutive frequency measurements. Then, the frequency protection can be adjusted accordingly. The momentary frequency variation is mainly dependent on the moment of inertia constant. For simplification, the damping 
factor is assumed to be zero during the short time window for a small sample load perturbation. The data window is adjusted as 60 message frames per second from phasor measurement unit. A more generalized equation of the equation (5.3) is represented as:

$$
\Delta P_{\text {load }}\left(T_{s}\right)=\Delta P_{\text {mec }}\left(T_{s}\right)-\frac{2 H_{\text {tot }}}{T_{s}}\left[\Delta f_{1}-\Delta f_{0}\right]
$$

$T s$ is the sampling period, $\Delta f 1$ and $\Delta f O$ are the system frequencies at $T O$ and $T 1$.

\subsubsection{Real-Time Implementation of Agent-based Frequency Protection}

Synchrophasor applications explicitly deal with the system disturbances of the dynamic operation of complicated systems. In this study, PMU-enabled IEDs are used to perform inertia estimation. High sampled data acquisition is adjusted to obtain 60 message frames per second. A common GPS time source is used to synchronize voltage and current waveforms in a fast and time-synchronized way. PMU-enabled IEDs are connected to a PDC. A PDC can be considered as a station in a communication network, where PMUs populate the time-aligned measurements. The deployed PMUs in the system send acquired data with time-stamp information to the PDC. The collected data from a number of PMUs are sorted and correlated according to the time-stamp values. This enables a comparable real-time monitoring of the system with high precision sampling.

Two type of frequency protection functions were implemented. IED3.2 and IED2.1 are load feeder IEDs, which facilitate load shedding. IED3.1 and IED1.2 are the DER feeder IEDs equipped with under-frequency, and ROCOF frequency gradient protection functions. LSHDFRQ is the load-shedding logical node. FRPFRC is the frequency protection logical node, including the over/under frequency and ROCOF protection function. The pulse load was connected to the IED2.1 feeder. 421 PMU and 451 PMU are 
the station names of the synchrophasor IED1 and synchrophasor IED2, respectively, as introduced in Figure 5.2.

Before the pulse train, a small sample load was applied to determine the system inertia, possible frequency deviation and the protection settings in the system. In order to determine the inertia constant of the islanded microgrid, two consecutive frequency measurements were obtained from the phasor data concentrator for a sampling time. The first measurement was obtained before the sample load triggering, and the second measurement right after the sample load by capturing frequency deviation in the system. Using two consecutive frequency measurements, as per equation (5.4), the inertia constant was calculated. By using the inertia constant information, the pulse load agent determines if the pulse loading would excess permissible limits of the load-shedding (IED3.2) and frequency protection function (IED3.1 and IED1.2) values. Accordingly, when the pulse train was applied, pulse load agent (IED2.1) broadcasts the blocking GOOSE messages to subscribed IEDs. Figure 5.12 shows the current measurement of the synchrophasor-based IEDs during the applied pulse train in the autonomous microgrid with a period of 3 second.

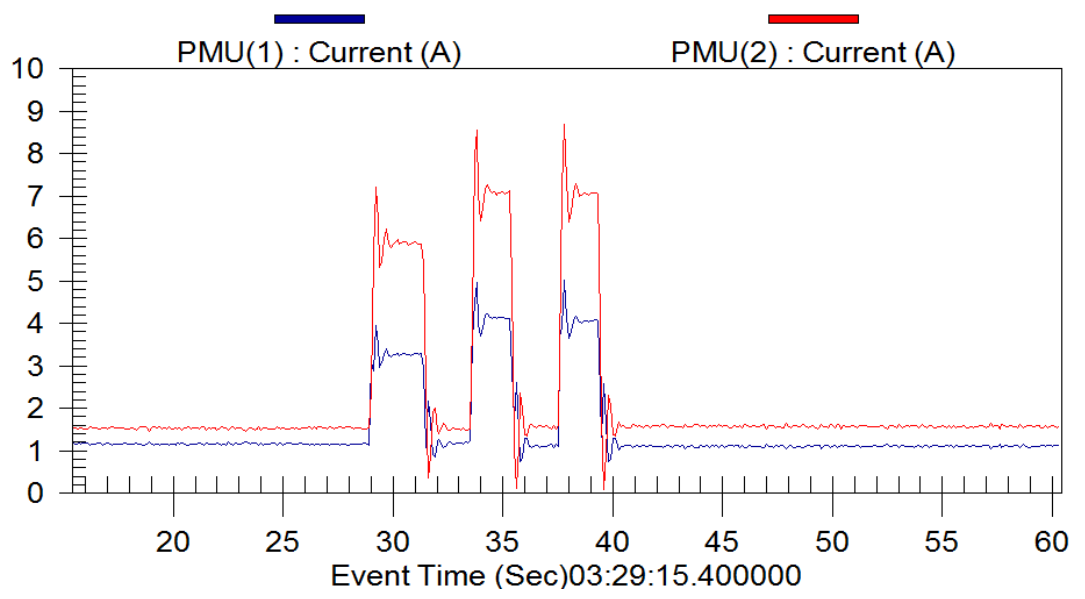

Figure 5.12: Power demand under pulse load. 


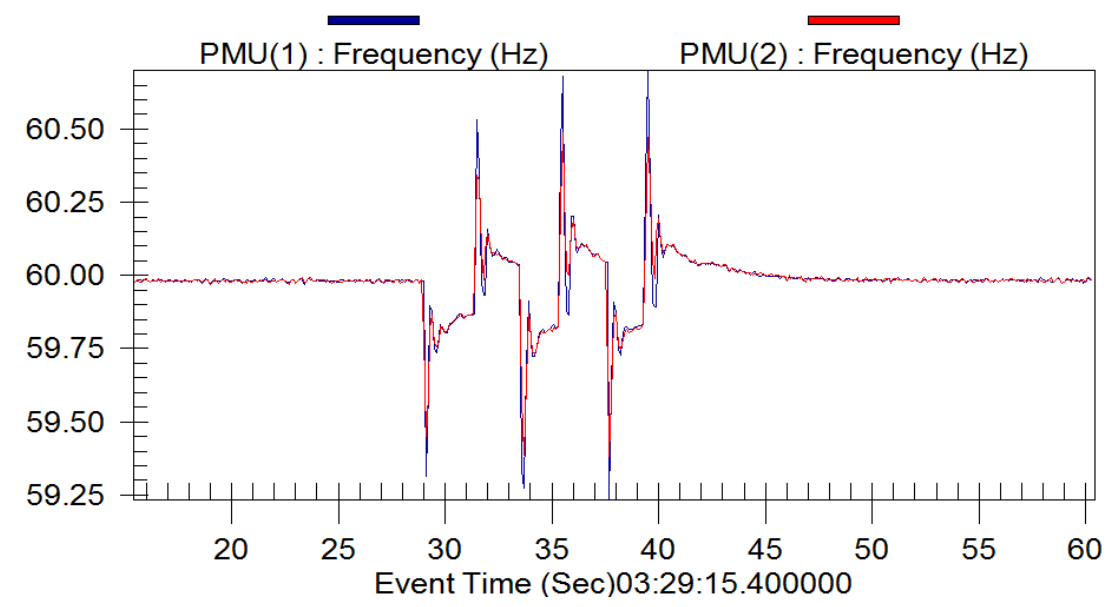

Figure 5.13: Microgrid frequency under pulse load train.

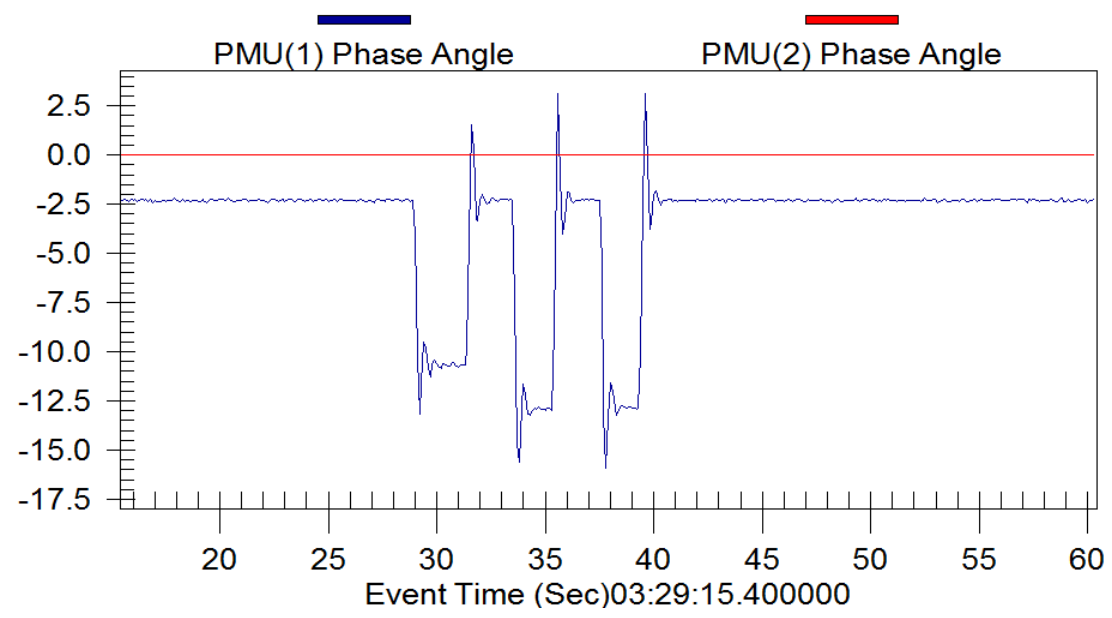

Figure 5.14: Phase angle difference between microgrid buses.

As the result of pulse loading, the voltage phase angles of the microgrid buses become far from each other. The phase angle displacement is shown in Figure 5.14. This situation would cause unintended frequency protection tripping of the ROCOF and VSR relays. To overcome this problem, when the pulse load train was applied, IED2.1 broadcasts the blocking GOOSE messages to the other IEDs as shown in Figure 5.15. 


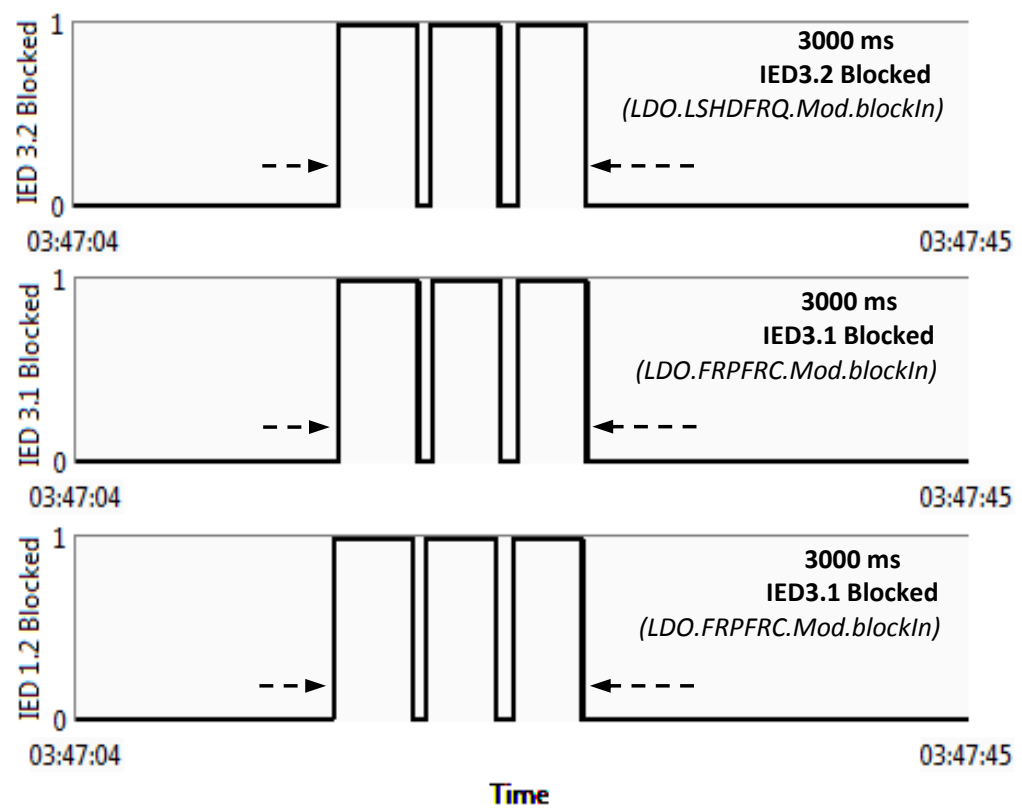

Figure 5.15: Blocked frequency protection and load shedding operation.

\subsection{Summary of the Results and Conclusion}

This chapter investigated the agent-based protection of autonomous microgrids with an industrial distributed communication IEC 61850 GOOSE protocol. Overcurrent and frequency protection challenges were assessed. A practical over current selectivity mechanism was introduced based on feeder characteristics and the location of the IEDs considering RAS. An online adaptive frequency selectivity approach was proposed to avoid pulse load effects in low-inertia autonomous grids. A state-of-the-art cyber-physical microgrid setup was introduced, including IEDs and actual power system components. Experimental results were demonstrated in order to validate the effectiveness of the proposed schemes. The results were found to be promising for actual field deployment of the challenging protection selectivity and RAS schemes in the low-inertia autonomous microgrid. 


\section{FULLY DISTRIBUTED APPROACH FOR AGENT-BASED PROTECTION OF MESHED MICROGRIDS}

\subsection{Introduction}

In this chapter, we present a distributed approach for microgrid protection with communication constraints, where each agent can only communicate with its neighbor agents. The study starts with identifying the future microgrid protection challenges, and introduction to distributed algorithms. Then, the application of a consensus-based auction algorithm for fault localization, and the clearing processes are discussed. A distributed depth-first search (DDFS) algorithm is introduced for adaptive dynamic agent discovery and network adjacency matrix reconfiguration after fault clearance. The next stage of the chapter includes simulation and experimental validation of the proposed algorithms

The traditional electric service mechanism is undergoing continuous changes with the increased penetration of utility independent autonomous private microgrids [97]. Investigations are underway on operating the existing distribution network as dispersed microgrids [98]. Aligned with the emerging smart grid philosophy, power distribution engineering must adapt itself using advanced protection methods as well [99].

The conventional power system protection requires radial protection devices connected in series, and the trip characteristics of the downstream protective device must be faster than the upstream devices. However, the integration of multiple DGs would form extremely complicated meshed microgrid networks with dynamically connecting / disconnecting DGs. This situation results in volatile network impedances, and accordingly 
stochastic fault currents. Thus, new challenges emerge by relying on predefined fault current settings on IEDs.

Traditionally, conventional protection schemes are operated for high current levels in distribution networks. However, in islanded microgrid operation, the high fault currents from the utility are not present [100]. In islanded mesh microgrids, various fault levels would prevail due to the wide range of short circuit impedances, and it is likely that multiple IEDs reporting fault locations for the same fault, yet actual clearing requirements are different [101]. The emerging challenges compel microgrids to adapt communicationbased cooperative methods.

In future smart grids, protection infrastructure is expected to be restructured as a cyber-physical system, including smart devices not only to carry power flow, but also to transmit data for protection via wireless and power line communications [102]. Distributed control architectures inherently rely on tight coupling between the cyber and physical layers, thus the networked information systems are the core components of microgrid interaction. In a dynamic network, the implementation of a centralized control with access to all IEDs in the system is not realistic. Major drawbacks of the centralized methods are the requirement of high communication capability with a powerful central controller, and being susceptible to single-point failure. This can easily jeopardize the system with a complete collapse. Due to their inherent resilience, agent-based distributed approaches have the ability to self-check and react accordingly to prevailing environment conditions even with communication constraints, where each agent can only communicate with its neighbor agents. In short, the future smart grid trend shows that advanced cooperative 
protection schemes and distributed communications are required to overcome existing microgrid protection limitations.

The related work in the area of microgrid protection is extensive. However, distributed algorithms have never been considered for protection engineering. Communication-assisted digital relays are proposed to overcome challenges during bidirectional power flow, looped feeders, and reduced fault levels in islanded operation [103]. Another communication assisted study proposed a zonal protection for ring microgrids specifically for shipboard power systems [104]. The adaptive coordination of the protection scheme under high penetration of DGs is discussed in [106], and the selection of alternative predetermined setting groups is demonstrated in [106]. A centralized protection scheme is introduced, but one that uses an online calculation method of the fault current coefficient, and time delay assignment for microgrid protection [107]. Agent-based distributed protection schemes are proposed using JADE [108] and combination of IEC 61850 object based modeling, and interoperable communication with IEC 61499 function block executable specification [109]. Both studies use distributed communication among agents, and they assume perfect communication in a static microgrid network with predetermined protection settings. In [110], a wireless communication network based microgrid protection scheme is proposed to allow relays to communicate to each other to dynamically track their tripping currents due to the timevarying nature of the loads and DG outputs. Recently, authors demonstrated a distributed communication based microgrid protection using GOOSE protocol [111]. Although microgrid protection solutions are evolving, distributed algorithms have never been 
exploited in the literature with communication constraints along with a dynamic network discovery.

\subsection{Microgrid Protection Challenges}

In this subsection, we address the challenges considering complicated meshed distribution network type.

\subsubsection{Distributed Approach for Microgrid Protection}

Major protection challenges of this type microgrids are:

- Due to various DGs kick in and out frequently, depending on the network topology, the short-circuit current is stochastically volatile.

- Any fault current is drastically dependent on fault impedance, whether it is a low or high impedance fault.

- Dynamic variation of the islanded microgrid frequency and voltage affects the fault impedance.

Considering the defined challenges, the protection schemes based on predetermined settings are not favorable. It is evident that there is a need for practical distributed localization on the fly. The leading motivations for adapting distributed communication through agents can be considered as:

- Resiliency requirements of the future microgrids call for wireless communication architectures that can provide alternative data routes in case of link failures.

- Microgrids would be located over a large geographic area with limited coverage area, allowing only neighbor-to-neighbor communication. 
The main challenges to implement distributed algorithms in real-time applications are:

- Power system protection requires high-speed clearing time, thus increasing the communication speed is crucial.

- Convergence time of the distributed algorithms drastically increase as the network size expands.

\subsubsection{Distributed Algorithms}

Distributed algorithms run in concurrent multi-tasking agent environments to accomplish tasks, such as communication, synchronization, resource management and consensus. The main interest is to be able to withstand failure of communication channels under uncertain timing, event order and data inputs. The environment is mainly defined as a dynamic network based on peer-to-peer communications, where participants may join, leave and move to other environments. The standard problems that have been studied are: leader election, consensus and distributed search. Leader selection aims to set special status capabilities to an agent for mainly scheduling tasks and allocating the resources. Distributed auction algorithms also utilize a market-based decision strategy as the mechanism for task assignment [112]. Consensus algorithm provides an agreement reaching solution in distributed systems, where each agent start the process with their initial opinion or values [113]. The distributed search algorithms offers a solution for node discovery in networks, where the topology of such networks changes continuously as new nodes join and old nodes leave the network [114], [115].

\subsubsection{Fully Distributed Selectivity Methods}

In this chapter, we propose a new approach for cooperative fault localization and node discovery. We consider that during the fault in a mesh microgrid network, the two 
adjacent IEDs detect the highest fault currents in comparison to the other IEDs in the network. However, due to the lack of global knowledge, the agents are not aware how high the other agents see the fault. Therefore, it is not possible to come up with a satisfactory fault clearing process without reaching a common agreement. It is assumed that each agent behaves in a greedy manner to clear the fault by opening their circuit breakers. However the satisfactory clearing is possible only when two proper adjacent circuit breakers actuate.

To overcome this problem, we first establish a dynamic leader selection mechanism using the multi-round consensus-based auction algorithm. The dynamic leader is the agent that detects the highest fault current in the network. Each agent node controls several current flow paths to different neighbors. Each agent determines the highest current among its controlled branches, and participates in the auction with this value. After the fault occurrence, the convergence of the first round consensus algorithm provides an average value of the total fault currents from all nodes in the network. Then, a continuous multiround auction mechanism is implemented until the leader agent is found. After the first consensus convergence, each agent compares its detected fault current to the global average value. If an agent's fault contribution is lower than the average value, it drops from the auction, but continues to participate in message conveying. The integrated consensus and reverse auction algorithm is repeated until the leader agent wins the auction. In the second part of the algorithm, the qualifier agent simply communicates with its adjacent agents to clear the fault through a direct peer-to-peer communication.

Once the fault is cleared, a graph information topology is required to adjust adjacency matrix coefficients according to prevailing network topology. Finally, a 
sequential distributed depth-first search algorithm is implemented to discover existing nodes through peer-to-peer neighbor communication.

\subsection{Implementation of Distributed Algorithms}

In this subsection, implementation of distributed algorithms for the proposed protection scheme is explained. First, we explain the average consensus method in order to come up with a universal agreement between agents. Then, we introduce a reverse auction mechanism to select the leader agent. Finally, the distributed depth search algorithm is presented for adaptive agent discovery.

\subsubsection{Average Consensus Method}

Consensus algorithm (protocol) is applied to multi-agent systems to reach an agreement with an information exchange between neighboring agents in the network. In our implementation, we exploit the consensus algorithm to provide an average value of the prevailing fault current information, so that an agent can make a decision whether the average value is exceeding its value or not. We assumed the mesh microgrid network is a dynamic undirected graph $G=(V(t), E(t))$ with the set of nodes $V(t)=\{1,2, \ldots, n\}$ and edges $E(t) \subseteq V(t) x V(\mathrm{t})$ as shown in Figure 6.1. The neighbors of the agent $i$ are denoted by $(t)=\{j \in V(t):(i, j) \in E\}$. The set of edges, and set of neighbors of every agent in a dynamic graph is time-varying. The diagonal degree matrix is $D i, j:=\{\operatorname{deg}(v i)$, if $i=$ $j ; 0$ otherwise $\}$ and contains the degree information of each vertex. The adjacency matrix $A=[a i j] \in \mathbb{R}^{n x n}$ represents the nonnegative weights that any node $i$ conveys to

communications received from node $j$, granted $j \in N i$ otherwise $a_{i j}=0$. A linear system in an undirected graph $($ aij $=$ aji for all $i, j)$. 


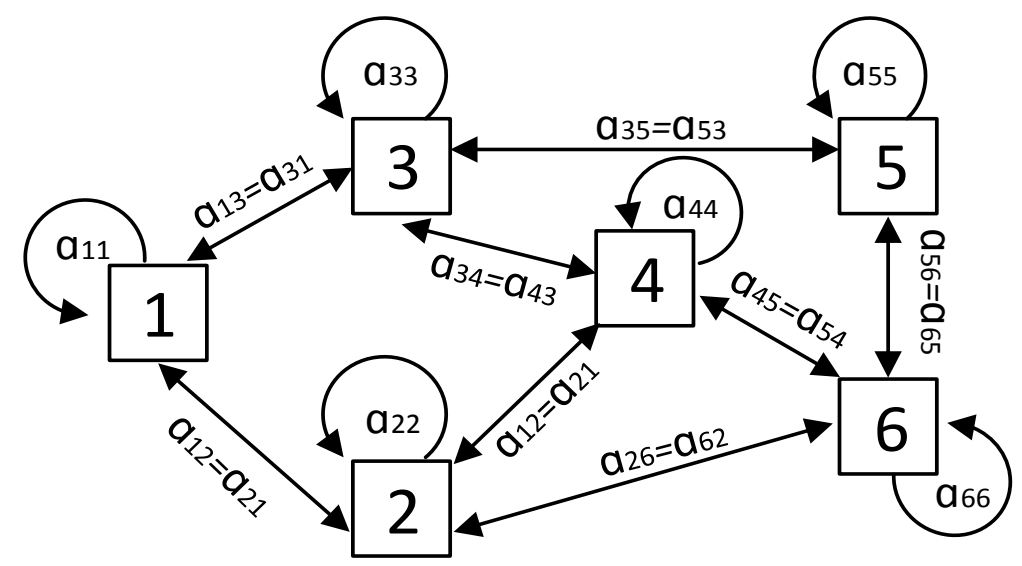

Figure 6.1: Meshed microgrid architecture

The representation of a consensus algorithm guarantees the convergence to a collective decision via distributed agent communication if the adjacent matrix is left stochastic. It yields the sum of the state of all nodes is an invariant quantity.

$$
X_{i}^{\prime}(t)=\sum_{j=N_{t}} a_{i j}\left(x_{j}(t)-x_{i}(t)\right)
$$

In particular, applying this condition twice at times $\mathrm{t}=0$ and $\mathrm{t}=\infty$ yields:

$$
\alpha=\frac{1}{n} \sum_{i} x_{i}(0)
$$

That is, when the consensus is reached, then the collective decision $\alpha$ is equal to the average of the initial state of all nodes. In our proposed protection algorithm, the initial states of the $X$ vector are the nominated overcurrent pick-up values by each IED node. The global average fault current would be for $n$ node graph:

$$
X\left[I f_{\text {ave }}\right]=\left[I f_{1}, I f_{2}, \ldots, I f_{n-1}, I f_{n}\right]
$$

The dynamics of such system in equation (6.1) can be expressed as:

$$
X^{\prime}=-L X
$$


$L$ is known as the Laplacian graph of $G$, which is defined as $L=D-A$. The Laplacian matrix is useful in analyzing the network stability, and performance through the eigenvalue of the matrix. The second eigenvector of the Laplacian of the $A$ matrix affects the speed of convergence. Thus, assigning the weights for the adjacency matrix is nontrivial, as the stability and performance of the system depends on it.

The Metropolis Rule in equation (6.5) is widely used for assigning weights, as it is doubly-stochastic (both row and column, or left and right, stochastic). It has been shown that it guarantees the stability and adaptation to topology changes with a near-optimal performance [116].

$$
a_{i j}=\left\{\begin{array}{c}
\frac{1}{\max \left(n_{i}, n_{j}\right)}, i \in N_{j} \backslash\{j\} \\
1-\sum_{i \in N_{j} \backslash\{j\}} a_{i j}, i=j
\end{array}\right.
$$

In other words, the weights from $i$ to $j$ depend on the maximum number of neighbors either node has, and the self-weight brings the total of each row (and column) in A to 1 . By recalculating the weights after the network changes in any way, the Metropolis rule provides the ability to adapt to changing topologies. For the graph in Figure 6.1, the weighted adjacency matrix using the Metropolis rule yields to equation (6.6). The adjacency matrix should be updated after each fault-clearing process since some edges would disappear from the network. The node discovery problem is solved by distributed depth-first search as explained in following sections. 


$$
\left|\begin{array}{cccccc}
1 & 1 & 1 & 0 & 0 & 0 \\
1 & 1 & 0 & 1 & 0 & 1 \\
1 & 0 & 1 & 1 & 1 & 0 \\
0 & 1 & 1 & 1 & 0 & 1 \\
0 & 0 & 1 & 0 & 1 & 1 \\
0 & 1 & 0 & 1 & 1 & 1
\end{array}\right| \text { yields }\left|\begin{array}{cccccc}
0.50 & 0.25 & 0.25 & 0 & 0 & 0 \\
0.25 & 0.25 & 0 & 0.25 & 0 & 0.25 \\
0.25 & 0 & 0.25 & 0.25 & 0.25 & 0 \\
0 & 0.25 & 0.25 & 0.25 & 0 & 0.25 \\
0 & 0 & 0.25 & 0 & 0.50 & 0.25 \\
0 & 0.25 & 0 & 0.25 & 0.25 & 0.25
\end{array}\right|
$$

\subsubsection{Reverse Auction Mechanism}

The reverse auction model is an efficient mechanism, where the environment consists of a single buyer and multiple sellers [117]. The result of the auction has an uncertain outcome, since the agents do not know other participants' bids. The winner is determined by running sequential auctions awarding a single task at a time until no other agent remains. In this work we exploit the reverse auction as a single task assignment among $N$ number of agents that maximize the global reward which is successful fault clearance. In centralized auction schemes, the bids are generally submitted to an auctioneer to determine the winner based on highest bids. The distributed auction algorithm proposed herein is merged with the consensus algorithm for conflict resolution without need of any auctioneer. Each agent determines to drop or stay in the auction by itself. The proposed consensus-based auction algorithm is a single assignment strategy consisting of iteration in two phases. First phase of the algorithm is the consensus algorithm providing a consistent situational awareness of the average fault current. The second is the reverse auction that determines which agents drop from the auction or which qualify for the next round. Table 6.1 illustrates the basic flow of the consensus-based auction scheme. 
Table 6.1: Consensus-based auction fault clearing algorithm

Begin

Initialize: A common threshold pick-up value is determined for all IEDs; Initialize the adjacency matrix and microgrid communication network;

if (If > If pick-up) then

Each agent: Nominate highest fault; Participate in consensus

First Average-Consensus: Microgrid agents: inform If1,..,Ifn ;

until (Leader agent is selected) do

if (Ifave > If) then

$\underline{\text { Agents: }}$ drop not participating for next round;

else

Agents: calculate $\Delta I f ;$ inform new Ifl,..,Ifn

end until

Leader agent: query-if adjacent IED to clear fault (aij=aji);

Leader agent: initialize node discovery (DDFS);

End

\subsubsection{Distributed Depth First Search Algorithm}

The DDFS algorithm is traditionally used for searching a tree or graph starting from a root node, and exploring the network. The main goal is to find the minimum spanning tree. In this work, we exploit DDFS for a complete node discovery in a network with a fully distributed fashion. Nodes are autonomous in a way that they perform their own computation and communicate with each other only by exchanging messages. Each node has a unique identity and neighboring information. The communication in the synchronous 
network applies the following assumptions: (i) all messages sent from $i$ to $j$ over the edge $(i, j)$ arrive without disruption in a finite time, however not necessarily in the same order they are published; (ii) each node is aware of its neighbors and identity of neighbors from which the message is received.

The discovery problem requires the existence of a unique node, called root, to initialize the algorithm. In our proposed method, we applied a dynamic root node, which is the leader agent elected after each fault clearance process. As in traditional DDFS, we use a sequentially token-passing method from node to node to explore the network. However, we adopted three different node information, and two message ontologies during the algorithm run time to define the agent's role in each time step.

An agent can be in discovered, visited and propagating states. An agent can be in multiple states at the same time. Propagate message ontology aims to send multicast messages to all neighbors to receive state knowledge, and discover the unexplored nodes in the network. Token pass ontology determines which agent is in charge to facilitate the node discovery in each round. Table 6.2 illustrates the basic flow of the distributed depthfirst search node discovery algorithm. All agents hold the state values for itself and neighbor agents. An agent turns into a discovered state following an acknowledgement message in response to receiving a propagate message from the token holder agent.

An agent is marked as visited right after the agent passes the token to a neighbor agent after the propagating process. The difference between a discovered and visited state is the agent's response to the propagate message. The reply of the discovered agent updates the online agent list and agent pair list. The agent list is an array holding the identity of the all discovered agents in the network. 
Table 6.2: Distributed depth-first search node discovery algorithm.

Initialize: Leader agent initiates node discovery; holds token;

Leader agent marks its self as discovered and visited.

Loop

Token Holder Agent: Propagate Neighbors;

Token Holder Agent: Token Pass to a random but not visited neighbor;

On receiving Propagate from $i=$ neighbor:

If (Propagate) then $\rightarrow$ return State

On receiving Token Pass from $i=$ neighbor:

If (Token Pass) then

mark Agent(i) as parent node

initialize propagate to neighbors;

On receiving Propagate Reply from $i=$ neighbor:

Switch Token Holder Agent do Agent's state

Case 0: Not visited nor discovered

Update both agent list and agent pair list;

Case 1: Discovered but not visited

Update only agent pair list;

Case 2: Visited

Skip;

Case 3: All neighbors are visited

Terminate discovery;

If (Terminate Discovery) then

until (Leader agent receives final lists) do

Agents: pass to parent nodes agent list and agent pair list;

end until 
On the other hand, the agent pair list holds information of links (edges) between adjacent agents. When the token is passed to the neighbor agent, the agent that received the token is marked as the child node, and the sender is marked as parent node. Upon completion of node discovery in the network, the final agent list and agent pair list returns to root node for adjacency matrix weight calculation using the Metropolis rule in equation (6.5). The root node is the first parental node, which receives the most updated list after the discovery algorithm termination. Figure 6.2 shows the first steps of the node discovery algorithm flow.

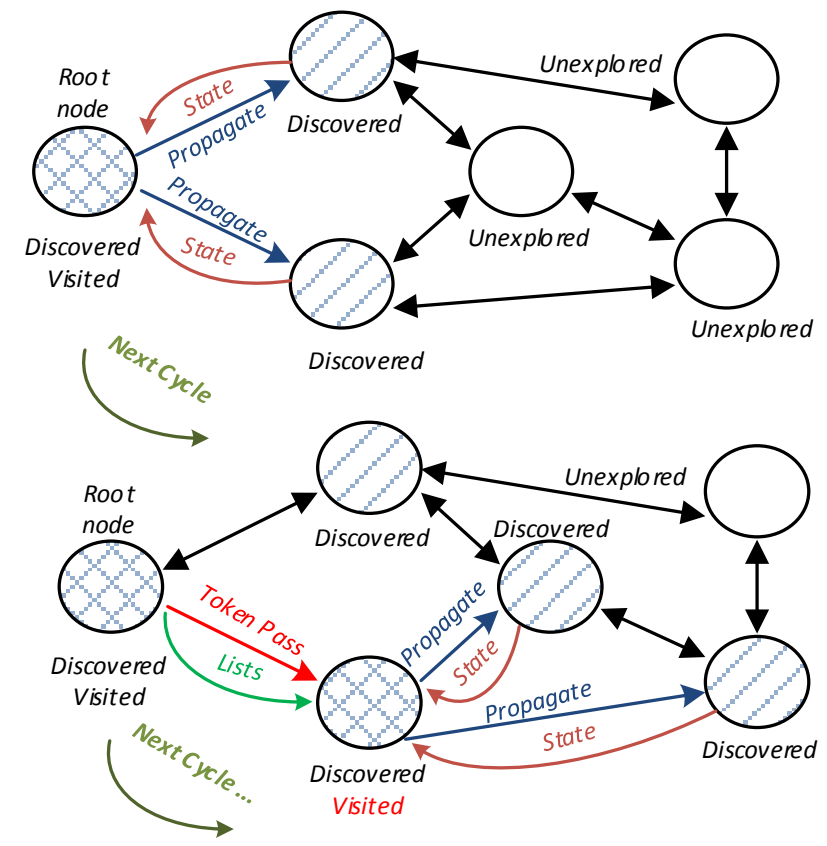

Figure 6.2: Node discovery algorithm flow

\subsection{Simulation and Experimental Results}

In order to illustrate the effectiveness of the proposed distributed algorithms, we first examined the results in a simulation environment. Then, an experimental real-time application on an actual power system setup is demonstrated. 


\subsubsection{Simulation Results}

A $2.4 \mathrm{kV}$ six-node industrial microgrid network, as shown in Figure 6.3, with line resistance and reactance $(R 1=0.025 \Omega / \mathrm{km}$ and $X 1=0.347 \Omega / \mathrm{km})$ was built in Simscape Power Systems [118]. Generator reactance and length of distribution lines are selected unalike to provide non-identical fault currents. Three different fault location are selected, as shown in Figure 6.3. Low impedance and high impedance faults are applied $(R f=0.01$ $\Omega, 0.1 \Omega$, and $1 \Omega$ ). Each node is modeled as an agent controlling multiple circuit breakers through separate current measurements.

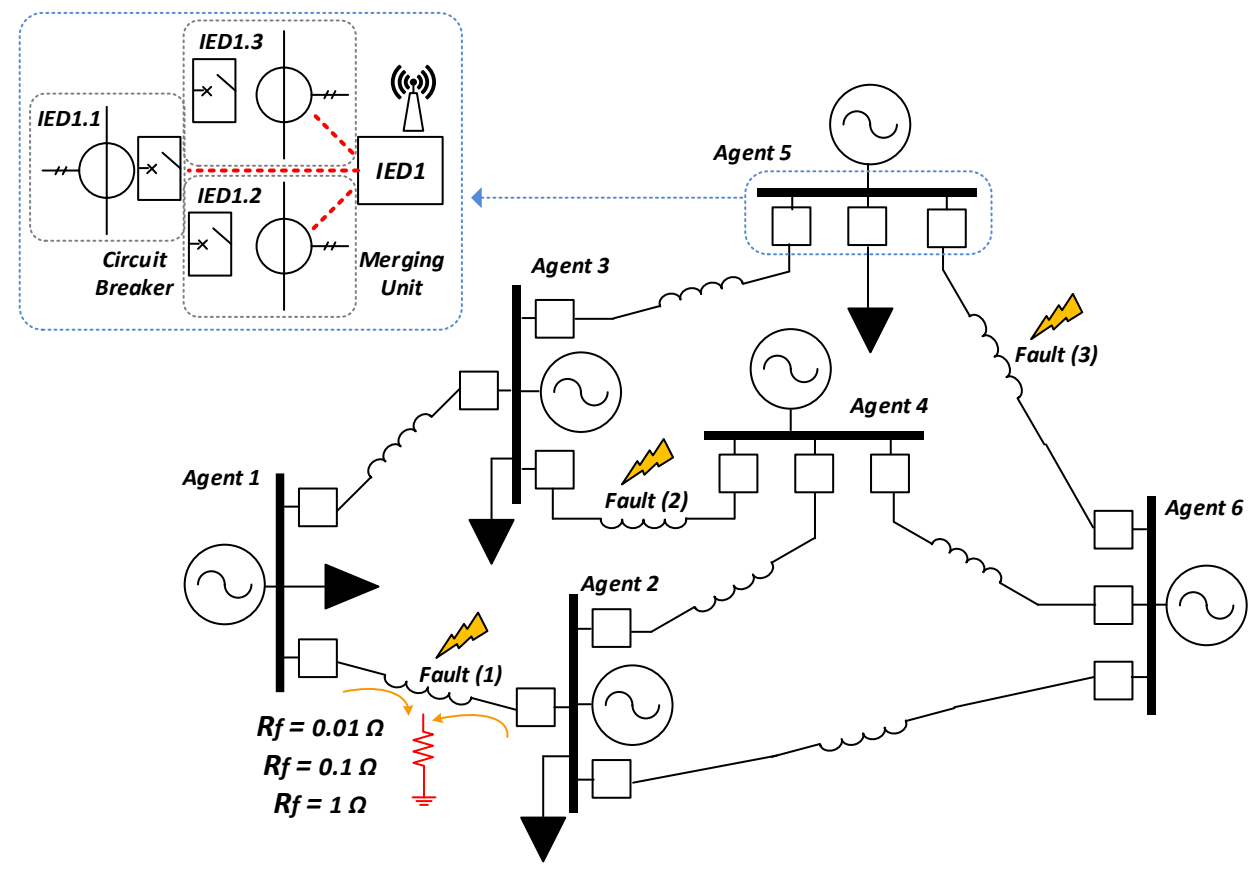

Figure 6.3: Meshed networked microgrid.

This can be assumed as an IED managing several merging units (MU) in the bay level, as shown in Figure 6.3. 

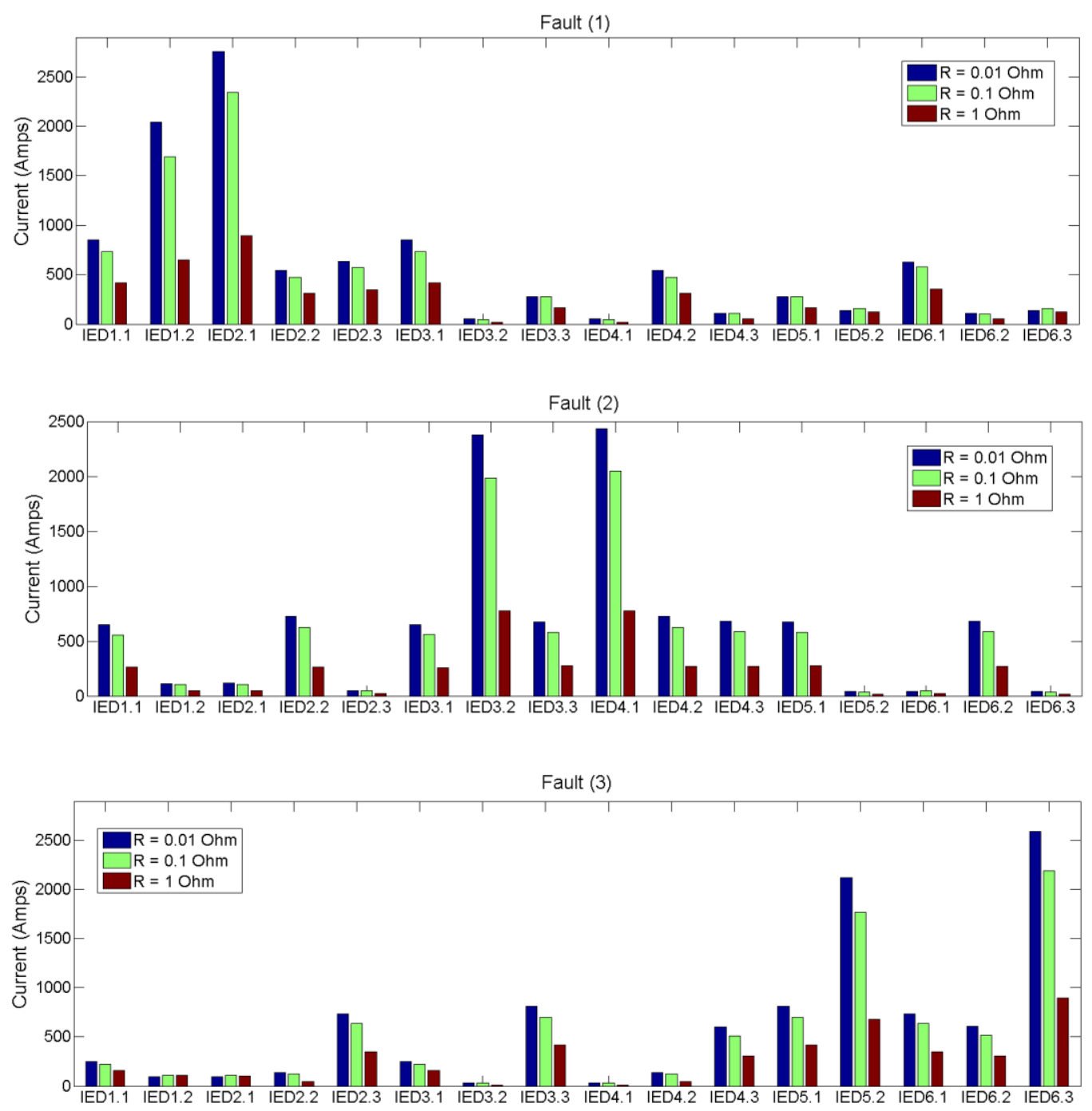

Figure 6.4: Fault currents in each node for three different fault impedance.

The fault currents observed in each MU node along with three different fault impedances are given in Figure 6.4. In each fault case, the IED located closer to the fault point detects higher fault current compared to any other node in the network. We demonstrated the proposed consensus-based auction algorithm for first fault point with Rf $=0.01 \Omega$. Recalling equation (6.3), the initial state vector $\mathrm{X}$ in this fault point would consist of the highest fault current seen by each IED. Accordingly, X[Ifave]= [IED1.2,IED2.1, IED3.1, IED4.2, IED5.1, IED6.1]. 


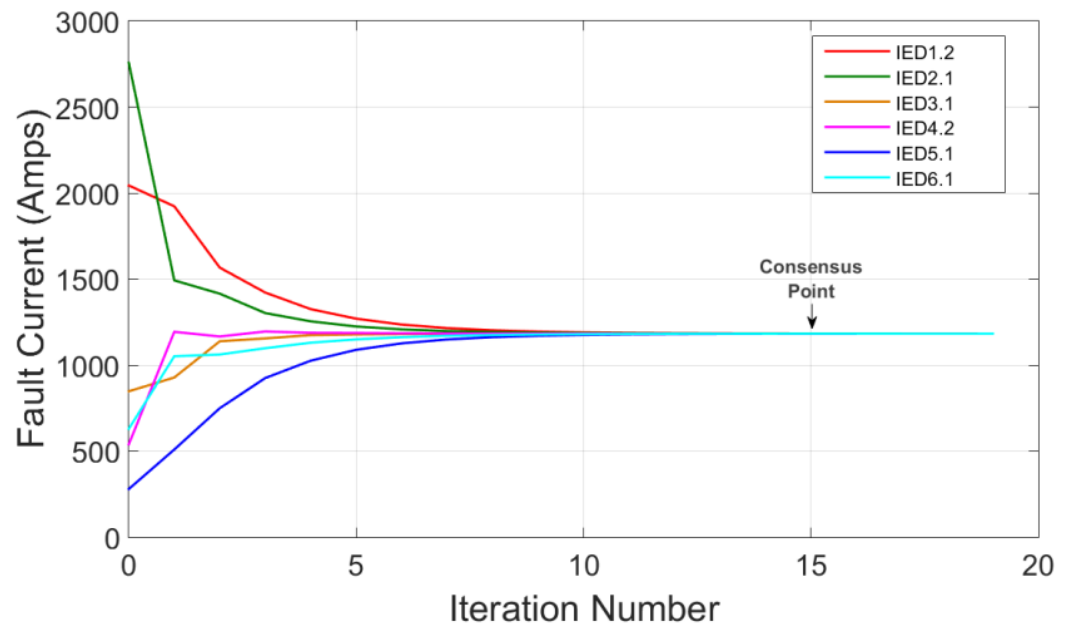

Figure 6.5: First average consensus result.

The consensus is reached perfectly around the $15^{\text {th }}$ iteration as $1183 \mathrm{~A}$. At the end of the first round, IED3.1, IED4.2, IED5.1 and IED6.1 drop from the auction. Their detected currents, 848 A, 540 A, 280 A, and 630 A, respectively, are lower than the average value. Only IED1.2 and IED2.1 remain in the auction with fault current values 2044 A and 2756 A, respectively. Figure 6.6 shows the second round consensus result. In fact, in this specific case, the qualifier IEDs of the first round determine the correct pair of circuit breakers to clear the fault.

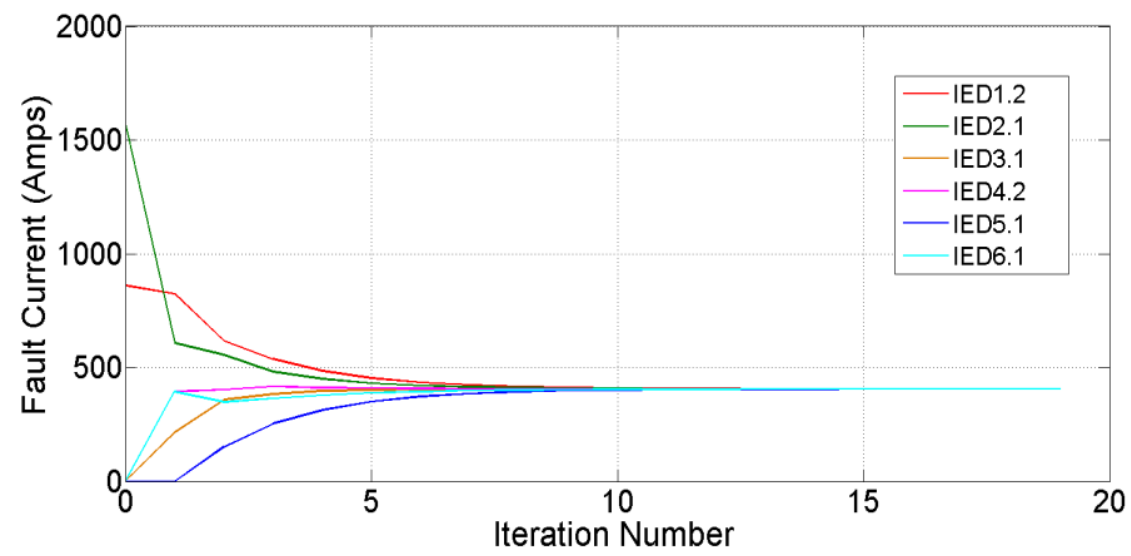

Figure 6.6: Second round average consensus result. 
However, to ensure robust decision making, the algorithm should continue until the leader agent is found. Thus, it can coordinate the clearing process with adjacent neighbors. If an agent remains in the auction, then it calculates three error values. The first error value $\Delta I f$ is the difference between the detected current and the consensus result. This is used by each agent to participate for the next round in the auction. The second error value $e$ is the error ratio given as $\frac{\Delta I f}{X[A v e]}$. The third value is the error deviation, $|\Delta e|$, from the previous round, which determines the termination of consensus-based auction algorithm resulting with the leader agent selection. When only the leader agent remains in the auction, the error deviation from the previous round is below a defined threshold accuracy. As shown in Table 6.3, the algorithm terminates at $7^{\text {th }}$ round, and the leader is elected as IED2.1.

Table 6.3: Results for fault point (1).

\begin{tabular}{c|c|c|c|c|c|c|c|c|c}
\hline \hline & Consensus & \multicolumn{4}{|c|}{ IED1.2 } & \multicolumn{4}{c}{ IED2.1 } \\
\hline \hline & X[Ave] & If & $\Delta I f$ & $e$ & $|\Delta e|$ & $I f$ & $\Delta I f$ & $e$ & $|\Delta e|$ \\
\hline $1^{\text {st }}$ Round & $\sim 1183$ & 2044 & 861 & 0.7278 & - & 2756 & 1573 & 1.3297 & - \\
\hline $2^{\text {nd }}$ Round & $\sim 406$ & 861 & 455 & 1.1207 & 0.3929 & 1573 & 1167 & 2.8744 & 1.5447 \\
\hline $3^{\text {rd }}$ Round & $\sim 270$ & 455 & 185 & 0.6852 & 0.2923 & 1167 & 897 & 3.3222 & 1.7773 \\
\hline $4^{\text {th }}$ Round & $\sim 180$ & 185 & 5 & 0.0278 & 0.2645 & 897 & 712 & 3.9556 & 2.1826 \\
\hline $5^{\text {th }}$ Round & $\sim 120$ & 5 & 0 & - & - & 712 & 592 & 4.9333 & 2.7507 \\
\hline $6^{\text {th }}$ Round & $\sim 99$ & 0 & 0 & - & - & 592 & 493 & 4.9798 & $\underline{0.0465}$ \\
\hline $7^{\text {th }}$ Round & $\sim 82$ & 0 & 0 & - & - & 493 & 411 & 5.0122 & $\underline{0.0324}$ \\
\hline \hline
\end{tabular}


In each consensus, approximately 15 iterations take place. This yields to a total number around 105 iterations until the leader agent is found. However, the total iteration number drastically increases as the network size expands. In Figure 6.7, we analyzed the consensus convergence in the famous IEEE 14 bus test system as well [119] to illustrate a comparison. The consensus is reached at around the $60^{\text {th }}$ iteration.

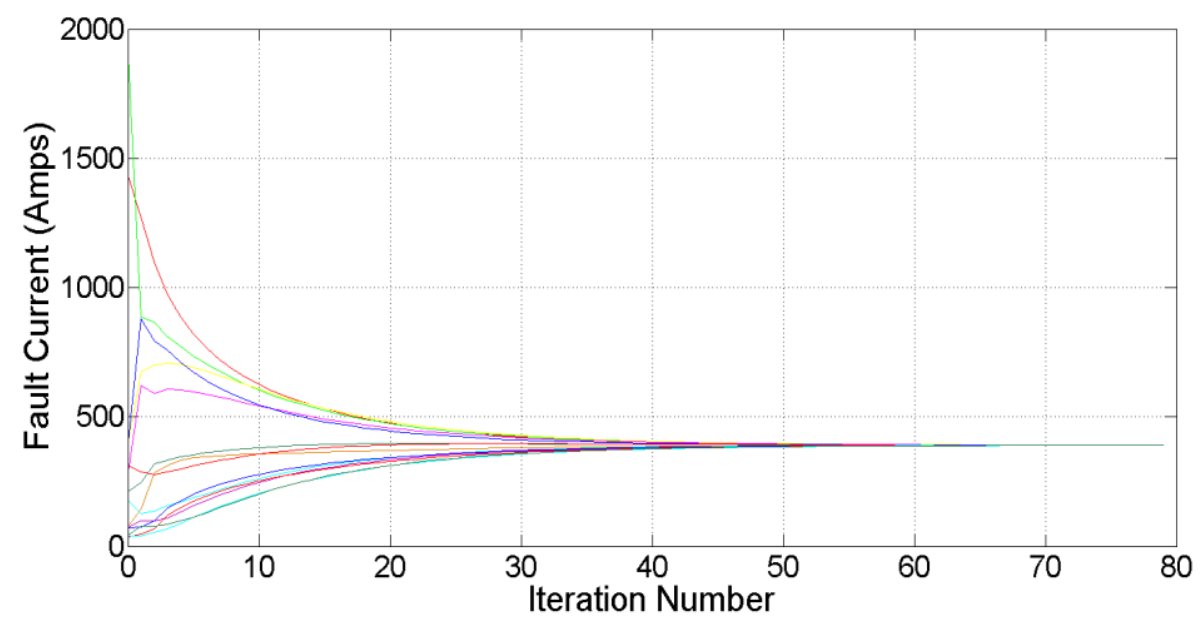

Figure 6.7: Consensus on IEEE 14 bus network topology.

\subsubsection{Experimental Results}

The simulation results provide proof of concept for the methods, however it is expected that proposed algorithms can be successfully applied to real-world microgrids. To illustrate the capability of the proposed algorithms, an experimental study has been performed in the microgrid setup. The reconfigurable power system was converted to a six bus meshed network microgrid. The IEDs are located on the system buses to enable protection and control. The IEC 61850 communication provides a standardized information exchange for power systems. However it is not sufficient to define behavior of the agents for advanced distributed algorithms. Therefore, semantics of the exchanged messages should be defined using a common knowledge representation. JADE implements 
agent communication language (ACL) to describe semantics of the messages. We implemented the agent framework to run distributed algorithms in real-time using IEC 61850 and the foundation for intelligent physical agents (FIPA) standards, as shown in Figure 6.8. The open connectivity unified architecture (OPC UA) is adopted as a middleware to acquire IEC 61850 logical node measurements, and the OPC UA client is embedded in the Java platform to enable JADE to access mapped IEC 61850 measurements. The agent platform is implemented in a personal computer, the information is accessible through the network, and the computation can be easily distributed.

\section{Agent Node}

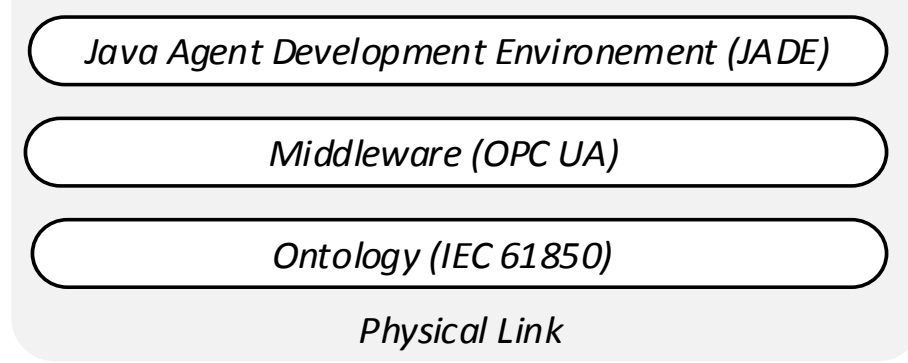

Figure 6.8: Agent node Architecture.

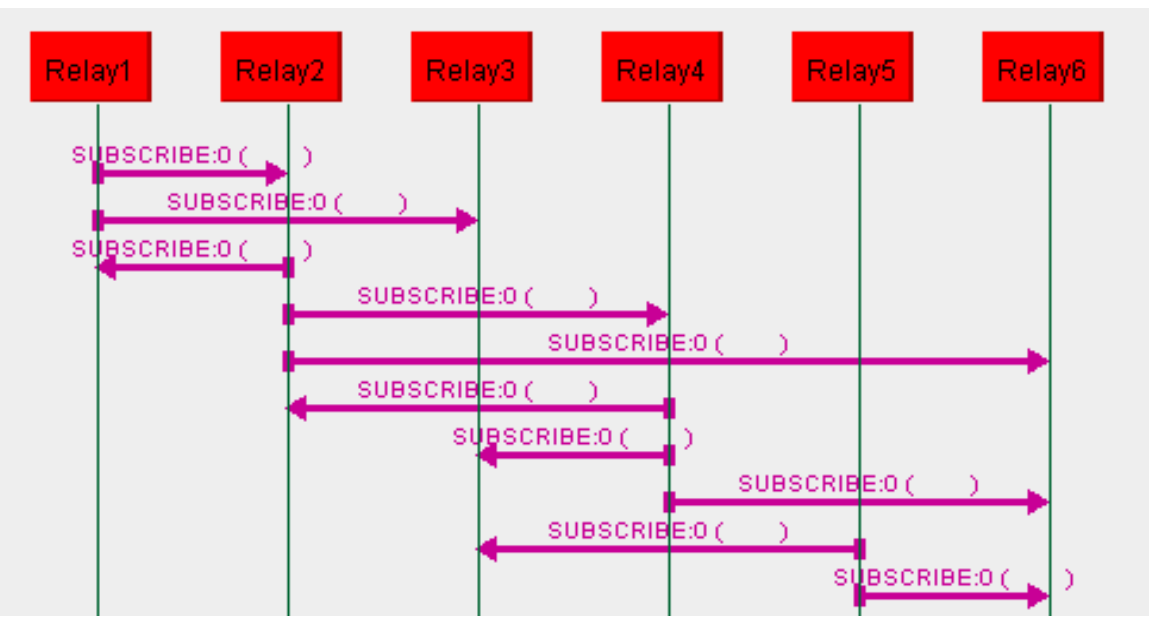

Figure 6.9: Agent subscribe to neighbors. 
The subscribe messages of the developed agents are shown in Figure 6.9. Once the agent platform is initialized, the neighbor agents subscribe each other to construct the communication network according to the graph. The communication capabilities of the agents are limited to only neighbor agents. The subscription process can be assumed as agents communicating through a wireless network only within the area of reach. To validate the proposed protection scheme, F1 scenario fault was realistically demonstrated. The fault was created with applying an $18 \mathrm{Ohm}$ high-fault impedance to the F1 fault point on the distribution line between IED1 and IED2. Figure 6.10. shows the fault current measurements of the IEDs during the fault instant. Once IEDs pick-up the fault current, the consensus algorithm initializes.

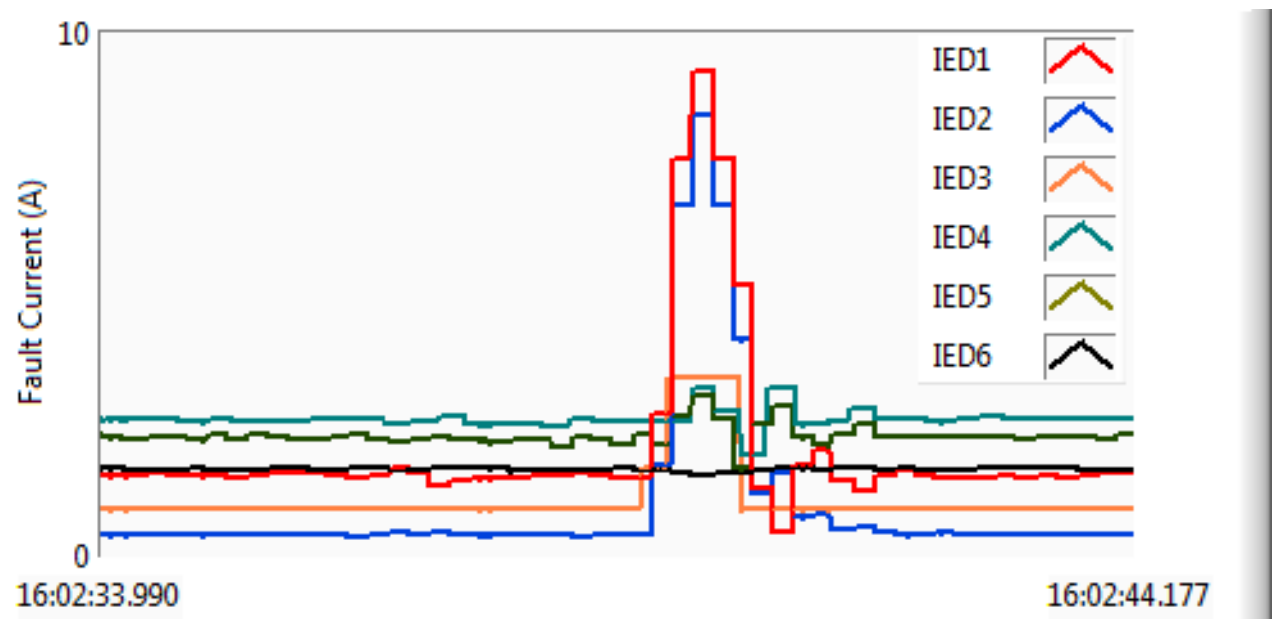

Figure 6.10: Fault currents of IEDs for F1.

The ontology of the consensus iteration messages was defined as inform. When the agents reach a consensus, they broadcast propose messages to the neighbors. As shown in Figure 6.11, Relay1 agent converged to the average value at the $232^{\text {nd }}$ iteration in the realtime experiment. The message handling rate of each agent was adjusted to 1 millisecond 
per message. This update rate does not consider communication delays in real-time communication media.

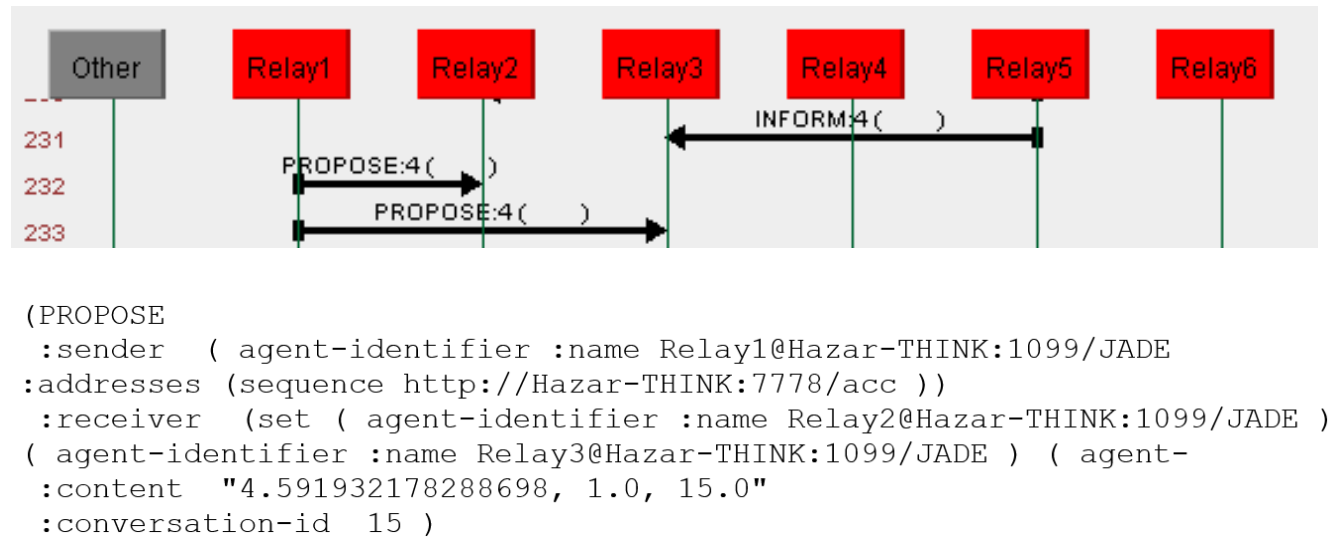

Figure 6.11: Relay1 convergence at $232^{\text {nd }}$ iteration and message content.

The exchanged message content is shown in Figure 6.12. The content of the message envelope is around 4.5919 A, which is the average value of the total fault currents of the agents. A precision accuracy was defined as $0.1 \%$ to determine the consensus decision. Convergence would be reached quicker if the accuracy is adjusted to be less accurate. The process is repeated until the leader agent is found.

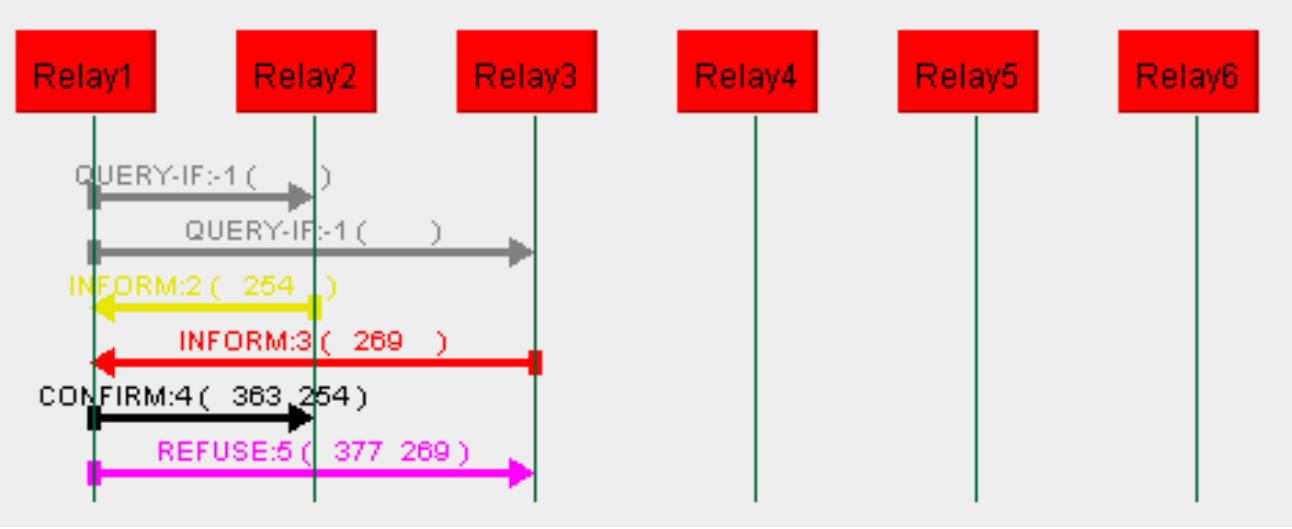

Figure 6.12: Relay1 communication with adjacent agents. 
Once the leader agent is elected, it broadcasts query-if messages to the neighbor agents to determine which adjacent agent should also open its circuit breaker, and clear the fault accordingly. This correspondence is shown in Figure 6.12. Relay1 queries neighbors for their detected fault current. Relay2 and Relay3 return first pick-up current value with an inform message. Relay1 determines the qualifier agent, and sends a confirm message. Refuse messages are sent to other neighbors, which are not qualified.

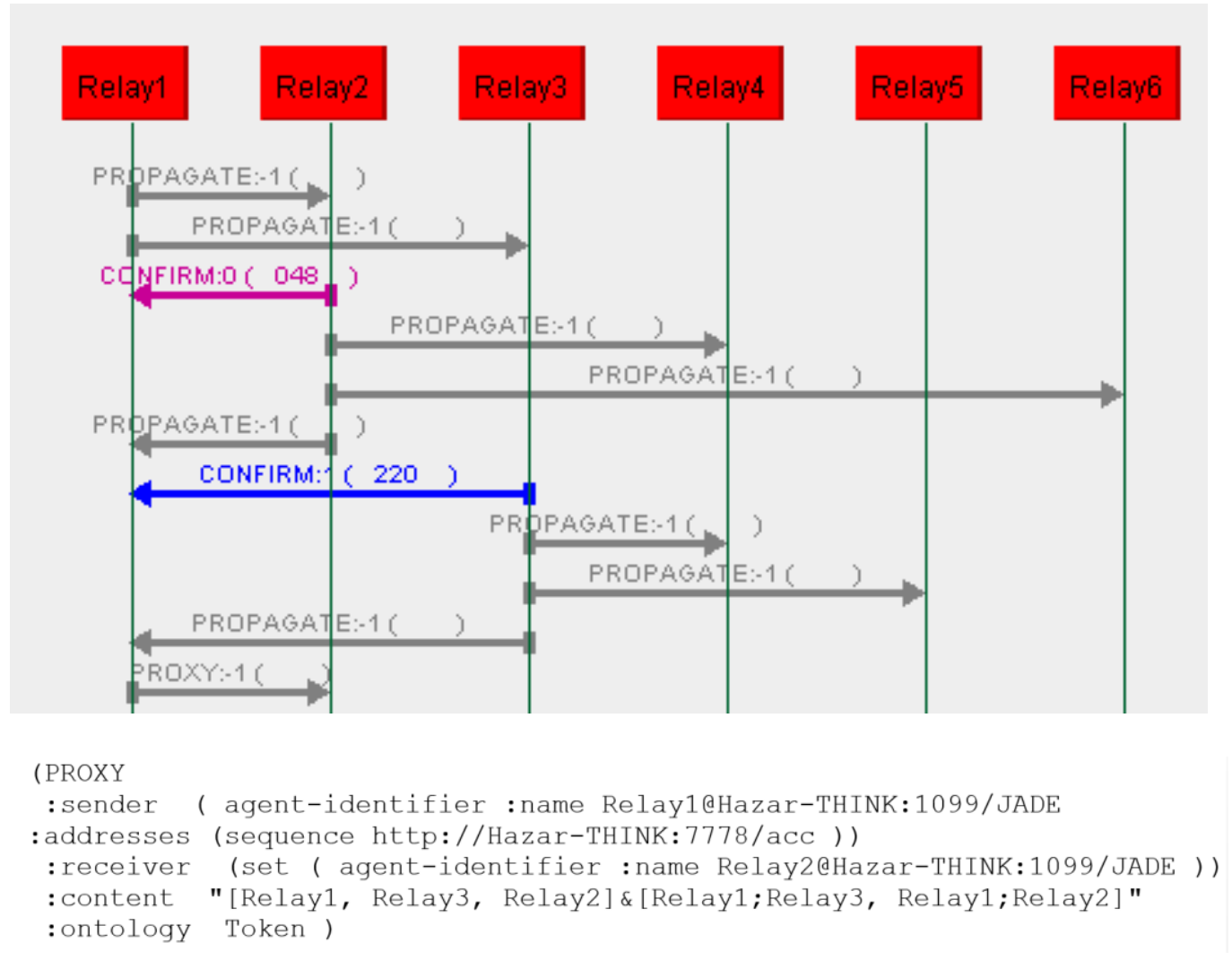

Figure 6.13: Relay1 agent initializes DDFS algorithm.

After the fault is cleared, the DDFS algorithm is initialized by the leader agent. Figure 6.13 shows the first round correspondence of the Relay1 agent until the token is passed to Relay2. Relay1 broadcasts propagate messages to the neighbors. The neighbor agents return their state along with confirm messages. Since Relay2 and Relay3 are just 
discovered but not visited, Relay1 updates both agent list and agent pair list. Once the propagation process is over, Relay1 passes the token to Relay2 agent through a proxy message. Upon receiving the message, the Relay2 labels Relay1 as parent node. The proxy message includes the most updated agent list [Relay1, Relay2, Relay3] and agent pair list [Relay1;Relay3, Relay1;Relay2]. The content of the message is also shown in Figure 6.13. The algorithm continues until the most updated list returns to Relay1 agent through its childe nodes in the network, and adjacency matrix is updated. The prevailing adjacency matrix is given as the below equation (6.7):

$$
\left|\begin{array}{cccccc}
1 & 0 & 1 & 0 & 0 & 0 \\
0 & 1 & 0 & 1 & 0 & 1 \\
1 & 0 & 1 & 1 & 1 & 0 \\
0 & 1 & 1 & 1 & 0 & 1 \\
0 & 0 & 1 & 0 & 1 & 1 \\
0 & 1 & 0 & 1 & 1 & 1
\end{array}\right| \stackrel{\text { yields }}{\longrightarrow}\left|\begin{array}{cccccc}
0.75 & 0 & 0.25 & 0 & 0 & 0 \\
0 & 0.50 & 0 & 0.25 & 0 & 0.25 \\
0.25 & 0 & 0.25 & 0.25 & 0.25 & 0 \\
0 & 0.25 & 0.25 & 0.25 & 0 & 0.25 \\
0 & 0 & 0.25 & 0 & 0.50 & 0.25 \\
0 & 0.25 & 0 & 0.25 & 0.25 & 0.25
\end{array}\right|
$$

\subsection{Summary of the Results and Conclusion}

This chapter presented a novel distributed protection approach for microgrids with communication constraints. First, a consensus-based auction algorithm was presented for fault localization. Then, a DDFS-based network topology adjustment algorithm was demonstrated. The main contribution of this chapter is to demonstrate the feasibility of microgrid protection in a fully distributed manner even with communication constraints. The proposed algorithm is immune to the high impedance fault detection problem, since only a simple pick-up current threshold selection is required for all IEDs. Furthermore, this method completely eliminates the time delay coordination, which has been traditionally used in conventional protection for decades. Our proposed protection method operates in a 
fashion that the quicker the cooperation among agents, the faster fault clearance is achieved. Although the proposed method requires further improvements to meet the highspeed fault clearance requirement, we anticipate emerging information and communication technology and distributed algorithms offer intriguing capabilities to reduce the convergence time and communication delays. Cutting-edge communication technologies and advanced distributed algorithms play a critical role on futuristic smart grid solutions, and protection schemes in particular due to the high-speed clearing requirement. IEC 61850 based generic object oriented substation event (GOOSE) technology enables fast horizontal peer-to-peer communication to exchange messages between devices with decentralized functions in around 3-4 ms [28]. However, this protocol should be extended with a semantic approach enabling further agent communication ontologies. Furthermore, IEDs using wireless and power line communication technology require further methods on communication delay handling. 


\section{DEVELOPMENT AND APPLICATION OF MICROGRID MULTI-AGENT INTEROPERABILITY TEST BED}

\subsection{Introduction}

This chapter presents the development and application of a real-time testbed for multiagent system interoperability. As utility independent private microgrids are installed constantly, standardized interoperability frameworks are required to define behavioral models of the individual agents for expandability and plug-and-play operation. In this chapter, we propose a comprehensive hybrid agent framework combining FIPA, IEC 61850, and OPC UA standards. The IEC 61850 logical node concept is extended using FIPA based agent communication language (ACL) with application specific attributes and deliberative behavior modeling capability. The OPC UA middleware is adopted to enable a real-time publisher-subscriber interoperability mechanism between platforms. The proposed multi-agent framework was validated in a laboratory-based testbed involving developed IED prototypes and actual microgrid setups. Experimental results are demonstrated for both decentralized and distributed control approaches. Secondary and tertiary control levels of a microgrid were demonstrated for decentralized hierarchical control case study. A consensus-based economic dispatch case study was demonstrated as a distributed control example. It was shown that the developed agent platform is industrially applicable for actual smart grid field deployment.

The traditional electric utility service mechanism is undergoing continuous changes with the increased penetration of utility-independent autonomous private microgrids. The 
major social and behavioral challenge is the integration of producer-consumers (prosumers) by providing incentives in the decision-making process. The main interaction of the prosumers and grid operators is embodied through an efficient microgrid management. Microgrids are the small-scale decentralized electricity networks featuring internal generation and distribution with individual priorities and operational behaviors. Prosumers must be equipped with an information framework and must be aware about the consequences of their actions, thus to create control architectures similar to social networks in order to achieve a higher quality management. In order to manage the complex microgrid structure, three different network types can be defined, as shown in Figure 7.1.

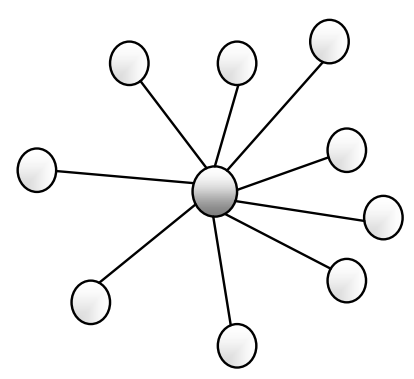

Centralized

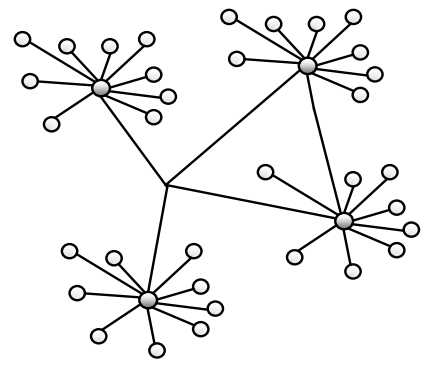

Decentralized

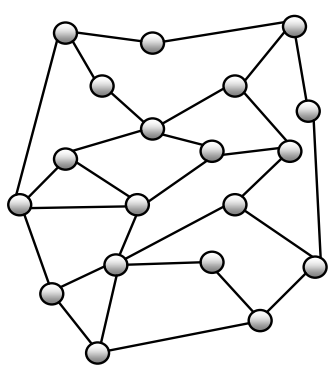

Distributed

Figure 7.1: Network types for microgrid control.

Centralized methods of operation are susceptible to single-point failures (master node), where managing the vast number of data generated from the extensive deployment of smart devices becomes infeasible. On the other hand, distributed approaches do not require a central station for control, and agents work autonomously in a cooperative fashion to reach a global objective. Cooperative control requires a tight communication among agents in a network, where the information sharing becomes the major challenge. Most of 
the time, distributed approach refers to a network, where each agent can only communicate with neighbor agents. Decentralized hierarchical control is a trade-off between two distinct controls approaches; centralized and distributed.

The hierarchical operation of the microgrids is proposed in the literature [120][121] which requires three control levels: primary, secondary, and tertiary according to (i) speed of response and information update time (ii) communication infrastructure requirements [122]. Each hierarchical level requires specific intelligence, optimization and behavioral modeling. In contrast to centralized control, the emerging smart grid concept compels the adoption of decentralized and distributed methods as a result of the highly dynamic behavior of the microgrids. Decentralized and distributed control approaches intend to provide autonomy for different control layers by enabling an event-driven peerto-peer communication structure, where central control schemes mainly rely on masterslave interactions [123]. The implementation of both decentralized and distributed controls are established using multi agent frameworks, which are composed of interacting multiple intelligent agents to achieve a global or local objective function. Embedded decisionmaking algorithms and individual behaviors facilitate the benefit maximization of the agents' autonomy.

Thus far, multiagent-based microgrid studies have been reported mainly in simulation environments [124]-[125]-[126], where the real-time information handling and data interoperability of the cyber-physical components have never been an issue. In reality, an agent is required to interact with its environment through sensors and actuators. A sensor acquires the data from the outside world and the actuator responds according to the agent's decision. For actual implementation of decentralized and distributed control schemes in 
power systems, it is imperative to link multi-agent objects to industrial control systems. The required interface is established through a combination of interoperable data and protocols.

IEC 61850 is the new international standard of communications, which enables the integration of all substation functions, such as protection, control, measurement and monitoring. IEC 61850 expands the area of influence in many parts of power systems due to its wide industry acceptance. The communication systems for hydroelectric power plants and DERs have been recently applied to other domains as IEC 61850 extension standards. Attempts to extend IEC 61850 protocol with IEC 61850-7-420 for DER control are promising [127], however the microgrid management concept covers extensive control, automation and protection applications, such that a single standard cannot meet all the required forms of monitoring and information exchange.

Decentralized and distributed control of the microgrids require interaction with utilities and internal DERs for dynamic management of the primary, secondary and tertiary control levels. Furthermore, decentralized and distributed controls require embedded optimization, behavioral modelling, implementation of artificial intelligence tools, and cooperative mutual negotiations of utility-independent entities. The FIPA is an organization that intends to evolve inter-operable agent communications with semantically meaningful messages, such as how messages are transferred and presented as objects [128]. The flexibility and implementation of behavior acts with ACL messages facilitates tailormade agent implementations [129]. 


\subsection{Hierarchical Microgrid Management}

Decentralized hierarchical control includes three distinct control levels according to the control response speed and communication infrastructure requirements. The primary control level deals with output power control of each individual DER unit and protection applications, which are based on local measurements. For example, droop control does not require decentralized communication. The secondary control deals with the economical and operational reliability of the microgrids. Agent based communication approaches are well-suited for decentralized controls in the secondary level for cooperation inside the microgrid, especially for stand-alone systems. The secondary control is achieved by DER units, which are generally located in the same microgrid, and are not widely dispersed.

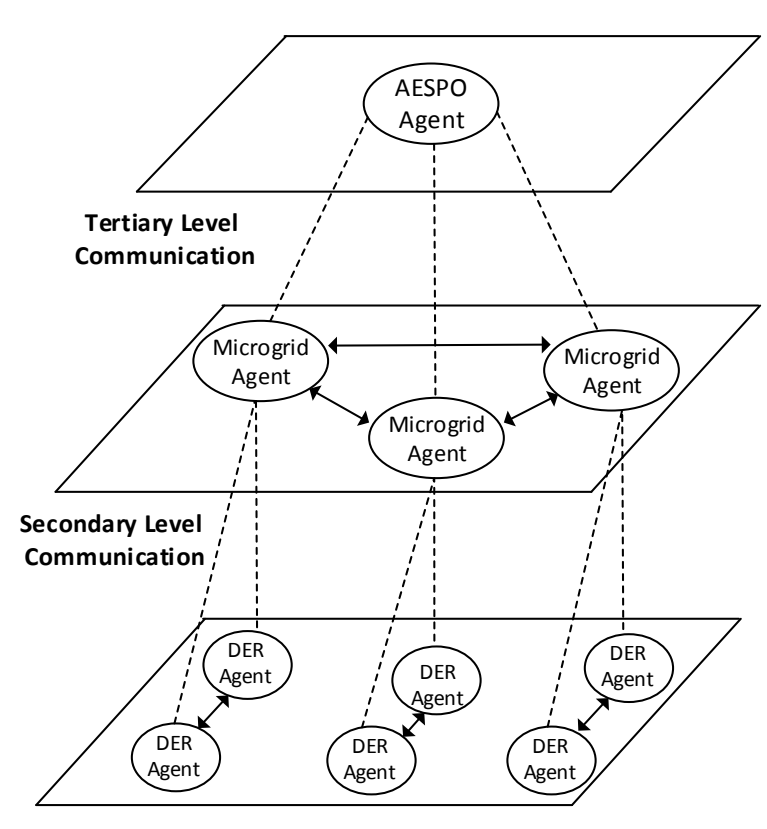

\section{AESPO Agent}

- Responsible entity of the host grid

- Interacts with microgrid agents

- Leads tertiary level controls

- Update rate: Minutes

- Ancillary service, Pricing (Auction)

Microgrid Agent

- Responsible entity of the microgrid

- Interacts with DER agents

- Leads secondary level controls

- Update rate: Seconds

- (e.g AGC, Optimization)

\section{DER Agent}

- Responsible entity of the DER unit

- Interacts with DER agents

- Lead primary level controls

- Update rate: Immediate

- (e.g Power sharing, Protection)

Figure 7.2: Hierarchical control of a microgrid.

Tertiary control can be assumed as the interaction of multiple microgrids with a host grid. Aggregated cooperation of multiple microgrids enhances the reliability of the 
host grid with ancillary services such as voltage and frequency regulation, pricing mechanisms including auctioning and bidding processes. Since a vast amount of information is required for this highly complex system, decentralized methods are more favorable for this geographically dispersed system.

Distributed control in microgrids is an emerging concept to handle topological variations, facilitate the plug-and-play feature, global information discovery and easy scalability. Distributed algorithms fit well on the operation of microgrids with communication constraints for such systems using wireless or power line carrier communication technologies. Through a cooperative approach, DERs can reach a consensus to reach a commonly agreed global objective. Most of the distributed solutions in the literature implements consensus-based algorithms.

The IEEE guide 1547.3 defines DER interoperability issues by means of monitoring, information exchange, and control. Some use cases are demonstrated as business operations of the DERs and stakeholder entities with direct communication interactions [22]. In this study, as shown in Figure 7.2, we adopted a model in which three control levels are defined hierarchically and linked to appropriate agents: (1) area electric power system operator (AEPSO); (2) Microgrid operators; (3) DER operators.

AEPSO Agent: is the responsible entity for safe and reliable operation of the host grid. The complete utility grid model is the property of AEPSO. The tertiary level controls are handled by the interaction of AEPSO and Microgrid Operators such as ancillary service and the market mechanism process.

Microgrid Agent: is the main responsible entity for monitoring, dispatch and control of the units inside the microgrid. The secondary controls are handled by interaction 
of Microgrid Operators and DER Operators, such as optimization and automatic generation control (AGC).

DER Agent: is the main responsible entity for individual DER generation units. Monitoring, protection and primary control of the units are handled by DER operators, such as power sharing and protection.

\subsection{Multi-Agent Information System}

This section explains the hardware and data information model of the proposed framework shown in Figure 7.3.

\subsubsection{IEC61850 Framework}

Self-describing devices and object-oriented peer-to-peer data exchange capabilities are the most significant superiorities of IEC 61850 over the other common standards. Logical nodes (abstract data objects) are the main elements of the IEC 61850 objectoriented virtual model, which consists of standardized data and data attributes. The virtual model expresses a physical (logical) device and number of logical nodes.

Each logical node contains data elements (DATA), which are standard and related to logical node functions. Most of the data objects are composed of common data classes (CDC), involving basic data objects, status, control, and measurement. Each data element consists of a number of data attributes with a data attribute type (DAType), which belongs to functional constraints (FC). Figure 7.4. shows a sample anatomy of an object name for a breaker position value. A physical device is defined by a network address. 

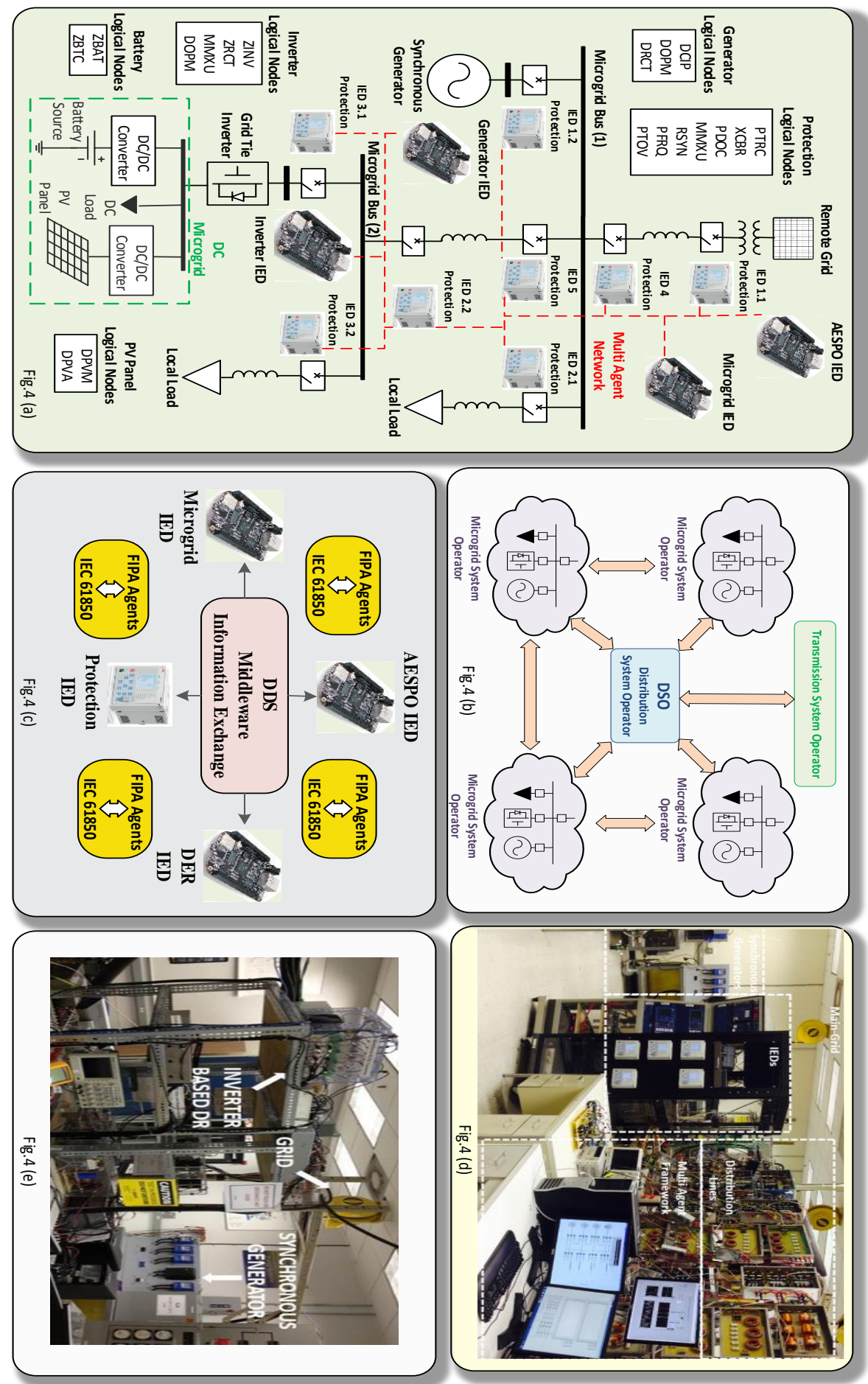

Figure 7.3: Cyber-physical infrastructure at FIU Smart Grid Test Bed. (a) Logical nodes and IED deployment inside microgrids. (b) Overview of network topology and decentralized bidirectional communication flow. (c) Prototype and commercial IEDs, multiagent information framework (d) Overview of the physical test bed network (e) Overview of a microgrid the setup. 


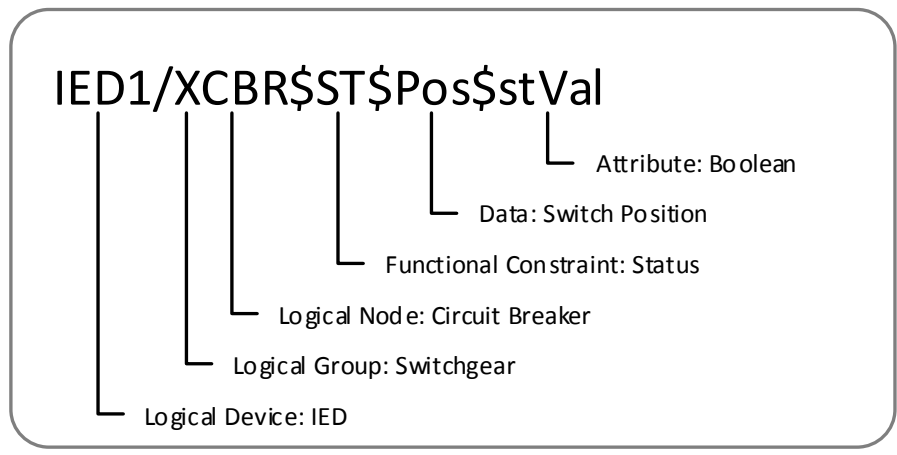

Figure 7.4. Object name of a circuit breaker position value

Generic object oriented substation events (GOOSE) is a multicast model based on a publisher-subscriber mechanism within the IEC 61850 framework, which ensures fast messaging with a $4 \mathrm{~ms}$ period of time. GOOSE messages are periodically sent from the publisher IEDs to subscribers with a To retransmission time period. Should an event occur related to GOOSE control, a new message is generated momentarily, then the message is continuously retransmitted with variable time periods $(T 1, T 2, . ., T n)$ until it reaches the To value again, as shown in Figure 7.5.

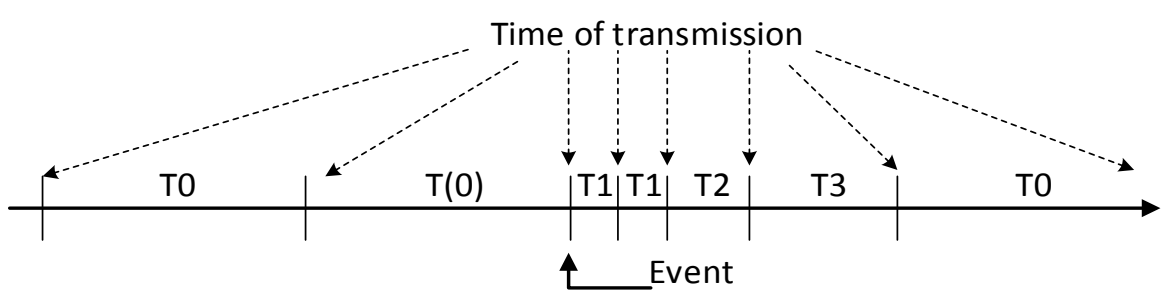
retransmission in stable condition (no events for long time) $\mathrm{T}(0) \quad$ retransmission in stable conditions may be shorter by event T1 shortest retransmission time after the event

T2,T3 retransmission time until achieving the stable condition time

Figure 7.5. GOOSE messaging.

This retransmitting scheme ensures the appropriate level of reliability. The fast messaging capability of the GOOSE model is widely used in modern power system 
protection applications, bringing forth a new era of advanced high speed peer-to-peer communication.

\subsubsection{FIPA Specifications and JADE Platform}

Agent communication language (ACL) represents a communicative act or messages intended to perform some action, with precisely defined syntax and semantics. An agent is an interacting object with its own thread of control that operates autonomously. Figure 7.6 shows a representation of a message exchanged between interacting agents. The beginning structure of an ACL message expresses communicative acts (inform, request, refuse etc.). Sender and receiver parameters designate the name of the sender and intended recipient agents, respectively. The content involves the object of the action and parameters passed through the message.

The message parameters define the expression of the agent responding to received messages, and which parameter is sent through the message. The JADE (Java Agent Development Framework) platform is based on FIPA specifications, which enable the creation of complex agent based systems with a high degree of interoperability using ACL messages. The JADE agent, at its simplest form, is a Java class that extends the core agent class which allows it to inherit behaviors for general management, configuration and registration of agents. The send and receive messages can be implemented by calling basic methods using standard communication protocols and registering in several domains. External software can be integrated by the use of behavior abstraction, which enables a link with OPC UA, along with the agent messages. 


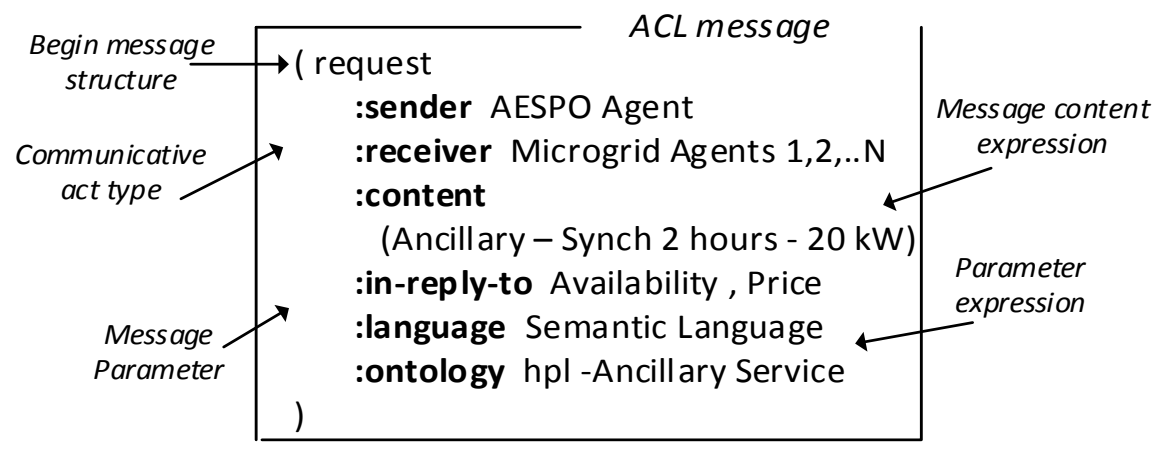

Figure 7.6: ACL message component.

\subsubsection{Open Connectivity Unified Architecture}

OPC was originally utilized to abstract various PLC protocols into an interoperable interface for a secure and reliable data exchange. The advent of smart grid interoperability efforts led to the development of OPC UA, which keeps all the functionality of the original OPC Data Access (DA), but switches from Microsoft-COM/DCOM technology to stateof-the-art web services technology. OPC UA is not directly compatible with the classic OPC since they use different technology. OPC UA uses a framework based on client and server architecture, in which the server provides real-time data to clients. Moreover, it can be implemented with Java or .Net platforms, eliminating the need to use Microsoft Windows-based platforms. This provides a perfect opportunity to model multi-agent based systems on Unix/Linux systems. The OPC UA modeling is based on nodes and references between the nodes. A node can have different sets of attributes connected through references. A nodeclass is composed of objects, variables and methods. A variable contains the value, in which clients can read, write and subscribe to the changes of the value. A method is similar to a function called by client and returns a result. The OPC UA address space is structured with objects containing only the node attributes, as shown in Figure 7.7. 


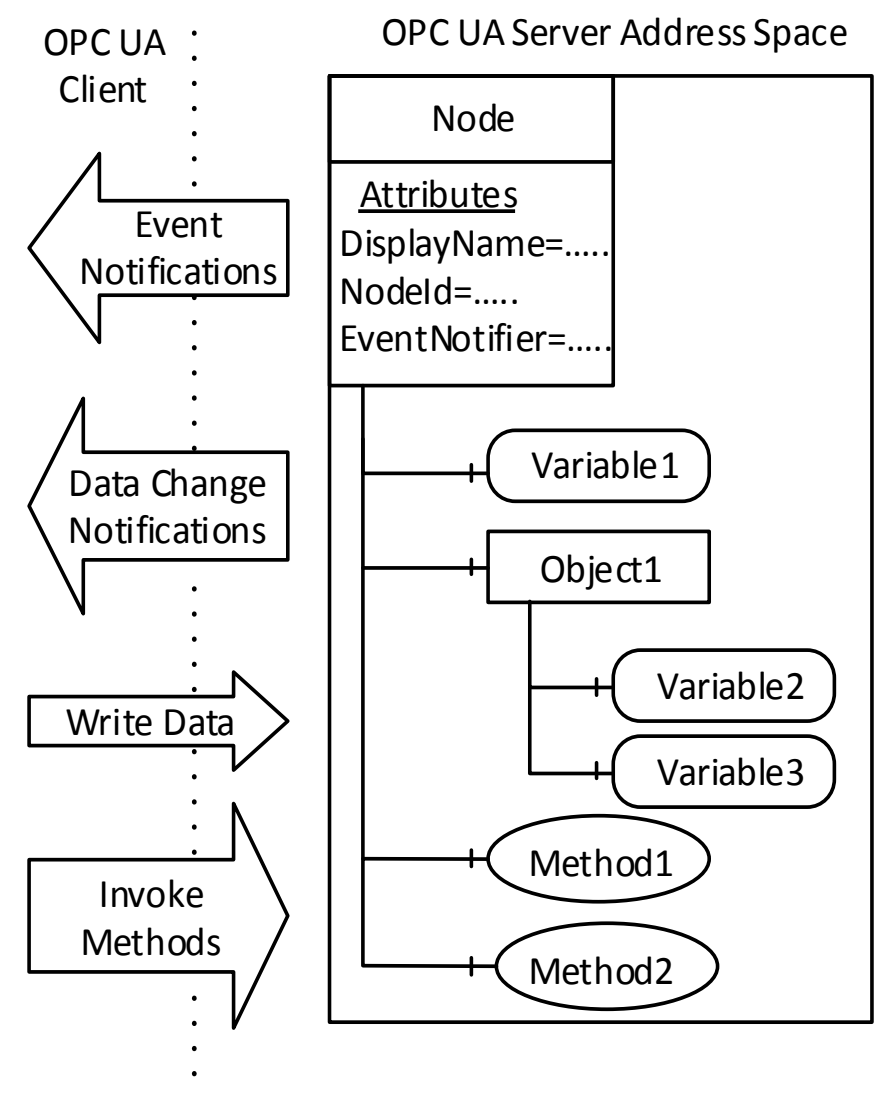

Figure 7.7: Microgrid Architecture.

\subsubsection{Developed Prototype IEDs}

The developed IED prototypes are designed to emulate real world hardware including all software layers. The hardware is based on the Sitara AM35xx chip form TI which provide high performance ARM processor and a two-slave 32bit microcontroller programmable real-time unit (PRU). The main processor is utilized to manage the communication, user interface, data logging and high level software layer. The PRU are utilized to handle real-time task and fast IO operation and data acquisition. Linux is selected as the operating system to manage the HW resources and provide networking stack. 
Linux has been chosen considering that it is open source and widely used in modern embedded systems and many commercial IEDs. For OPC UA implementation, an opensource library is used and compiled to work on the embedded board. For the IEC 61850 the open source $\mathrm{C}$ libiec61850 is used. A software application was developed to bridge the information between IEC 61850 and the OPC UA domain. All IEDs were connected to the network using standard Ethernet interface.

\subsubsection{Microgrid Management Test Setup}

The hybrid microgrids involve a synchronous generation unit and an inverter based DER. In addition to the local loads, which are connected to the point of common coupling of the generation unit, the system has a global load on the main bus. The microgrid has a connection to utility grid, which enables both in grid-connected and islanded operation. Operational voltage level of the microgrid is $208 \mathrm{~V}$ rms line-to-line, which is the utility voltage level. A 13.8-kVA, 60-Hz, 208-V and $1800 \mathrm{Rpm}$ AC synchronous generator represents a conventional synchronous generation unit. Inverter-based DER is interfaced with the AC microgrid via an AC-DC/DC-AC converter to allow bidirectional power flow between AC and DC parts of the microgrid. A 6-kW programmable DC power supply and battery stacks are used to emulate typical renewable energy resources and storage.

\subsection{Behavioral Modeling and Case Studies}

This section introduces how behavioral modeling of the agents is established through the proposed multi agent framework and the experimental case studies for the validation. The main goal is to illustrate how agents are loosely coupled and interact with each other, unlike dictating function block schemes, but with an influence of their beliefs. 
In an event-based IEC 61850 function block, an agent does not have any other choice if an event is invoked and mandate to facilitate an action. However, in the proposed framework, an agent performs its own autonomous priorities, and not likely to do tasks just because any other agent demands.

\subsubsection{Decentralized Hierarchical Control Case Studies}

A tertiary level control is demonstrated with an ancillary service for load regulation in the host grid feeder and a secondary level control is demonstrated with an AGC inside a microgrid. In the ancillary service use case, AEPSO agent is responsible for safe operation of the grid and the microgrids intend to sell power to the host grid. The mutual benefit brings all agents in a social environment to cooperate and achieve a task with proper negotiations. In the AGC control use case, the microgrid agent is responsible for frequency and voltage control of the microgrid. Similarly, each DER has individual properties, such as a cost function and availability.

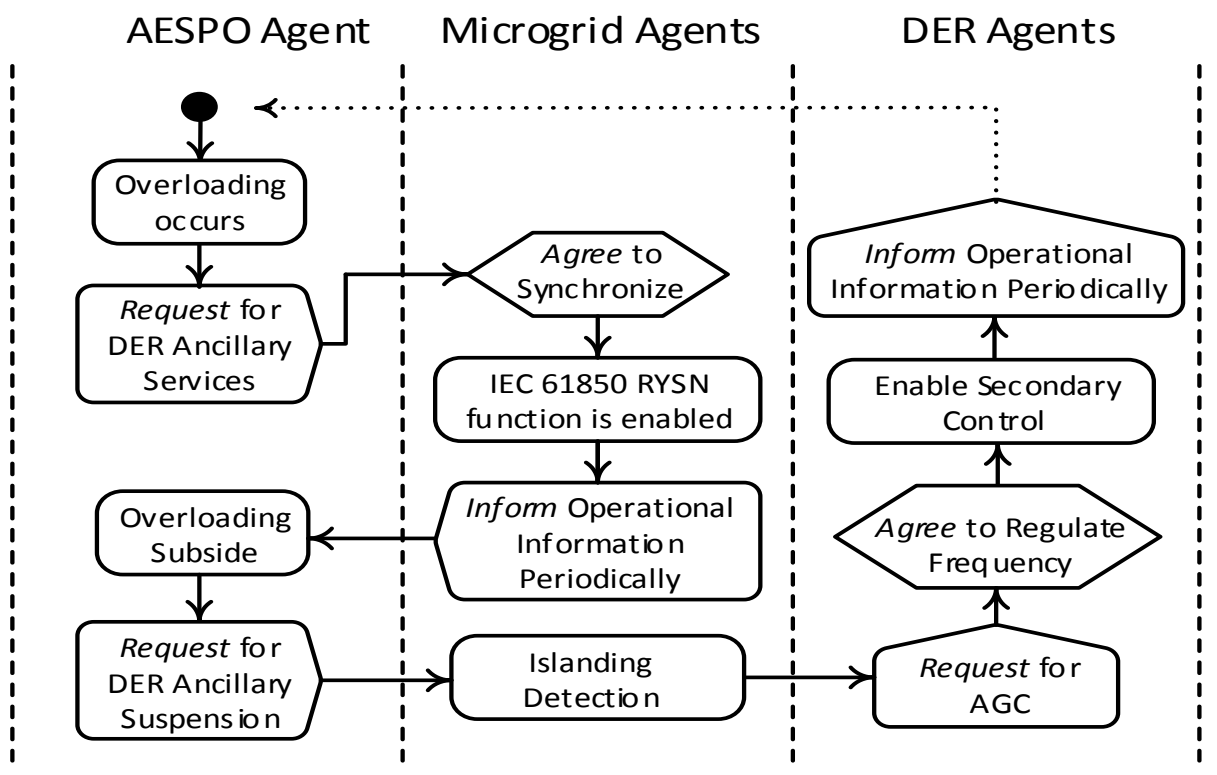

Figure 7.8: Case study flow chart. 
However, the ultimate goal is to keep the microgrid operational values in permissible limits. Figure 7.8 presents the case study flow and interaction of specific agents for secondary and tertiary controls. Figure 7.9 illustrates the behavior modelling of hierarchical agents and ontologies defined for messages of various behaviors.

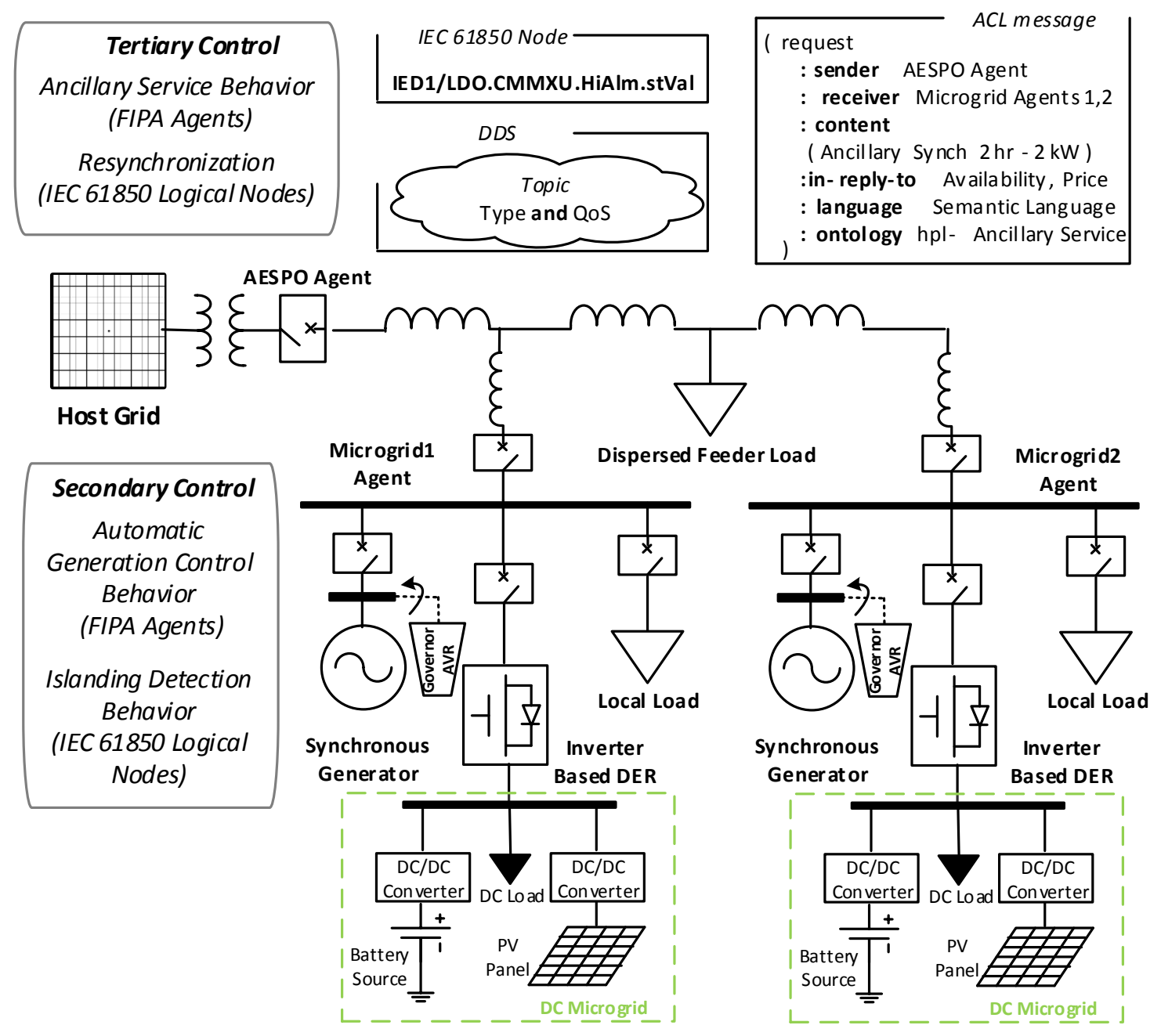

Figure 7.9: Implemented behaviors.

\subsubsection{Tertiary Control (Ancillary Service) Case Study}

Microgrids can be utilized to provide ancillary services, such as load regulation and reactive power support in distribution feeders. Especially during peak hours, the excessive 
energy demand may result in overloading of the distribution lines by drawing excessive current. This would result in thermal overheating and voltage drops beyond permissible limits on different parts of the feeder. Microgrids would provide a solution to relieve such overloading problems by contributing with either active or reactive power support.

In this use case, the AEPSO agent and microgrid operator agents are defined in the JADE platform and IEC 61850 logical nodes are utilized. The AEPSO agent is intended to continuously check the critical current flow value from the beginning point of the feeder through the IEC 61850 three phase current measurement CMMXU function block, which is a logical node inherited from MMXU for metering and measurements. When the current flow from the feeder reaches its critical value, the high-alarm node LDO.CMMXU.HiAlm.stVal of the function block becomes high. The Ancillary Service Behavior of the AEPSO is invoked and an ancillary service support Request message is published to microgrid operator agents registered to the directory service (yellow pages). Yellow page is a service mechanism in the JADE platform, in which an agent can find other agents providing the services it requires in order to achieve its goals.

The directory facilitator (DF) is the agent that provides yellow page service to the agent platform. The AEPSO agent periodically looks up available operators from the DF agent. The required amount of power is calculated based on exceeding the current level on the feeder. The main purpose of AEPSO agent is to purchase required amount of energy with the least cost. The aggregator agent collects the submitted bids and checks for the minimum cost. Each conventional generator has a quadratic cost function equation (7.1) which affects the cost of electricity according to volatile fuel cost for different hours, where " $i$ " unit number, " $P$ " electrical output and " $a, b, c$ " are random varying fuel coefficients. 


$$
\begin{gathered}
F_{c}(P)=\sum_{i=1}^{n} a_{i}+b_{i} P_{i}+c_{i} P_{i}^{2} \\
F_{\text {storage }^{2}}\left(S O C_{\text {Batt }_{i}}\right)=a_{i} / S O C_{\text {Batt }_{i}}{ }^{2} \\
F_{\text {Mic }}=\sum_{i=0}^{N} F_{\text {Gen }}\left(P_{G_{i}}\right)+\sum_{i=0}^{N} F_{\text {Storage }}\left(P_{S_{i}}\right)
\end{gathered}
$$

Renewable resources are coupled with storage devices. Inverter-based storage resources have a simple quadratic function with respect to the current state-of-charge (SOC) of the battery bank equation (7.2). The cost increases parabolically as the SOC reduces. In this study, we assume that the reserved price of a microgrid is the combined function of DERs, where SOCBatt is the state-of-charge of each battery bank, ai is the corresponding constant, Fstorage is the total generation cost of the battery bank and Fmic is the microgrid combined cost function equation (7.3).

AEPSO agent requests ancillary service and solicits bids from the available microgrid agents. Microgrids propose service with the prices. AEPSO agent sends accept/reject proposal messages to microgrid agents in return. Then, the qualifying agent replies with the confirmation message. As the result of negotiations, the microgrid agent which is qualified to provide ancillary service, enables the synchronization behavior. The synchronizer behavior is defined as the IEC 61850 synchronism check (RSYN) logical node of the IEDs and not defined in JADE platform. The synchronizer continuously checks the condition across the circuit breakers from bus and line regions of the power system and gives the permission to close the circuit breaker when the synchronization conditions are satisfied. Synchronization permission and circuit breaker closing signal is subject to frequency, phase angle difference and voltage values from both sides of the circuit breaker, 
where $f$ is the frequency, $\varphi$ is phase angle difference, and $T$ is the time duration equations (7.4) and (7.5).

$$
\begin{aligned}
& T\left(\left|f_{\text {host }}-f_{\text {microgrid }}\right| \geq f_{\text {threshold }}\right)=T_{\text {threshold }} \\
& T\left(\left|\varphi_{\text {host }}-\varphi_{\text {microgrid }}\right| \geq \varphi_{\text {threshold }}\right)=T_{\text {threshold }}
\end{aligned}
$$

Figure 7.10 (a)-(b) shows the frequency and phase angle difference of AEPSO and the microgrid. The figures cover 30 seconds of the synchronization process. Initially, the microgrid is operating at $61 \mathrm{~Hz}$. From the $35^{\text {th }}$ to $65^{\text {th }}$ second, the generator output frequency decreased manually by decreasing the applied torque to the generator shaft from the governor.
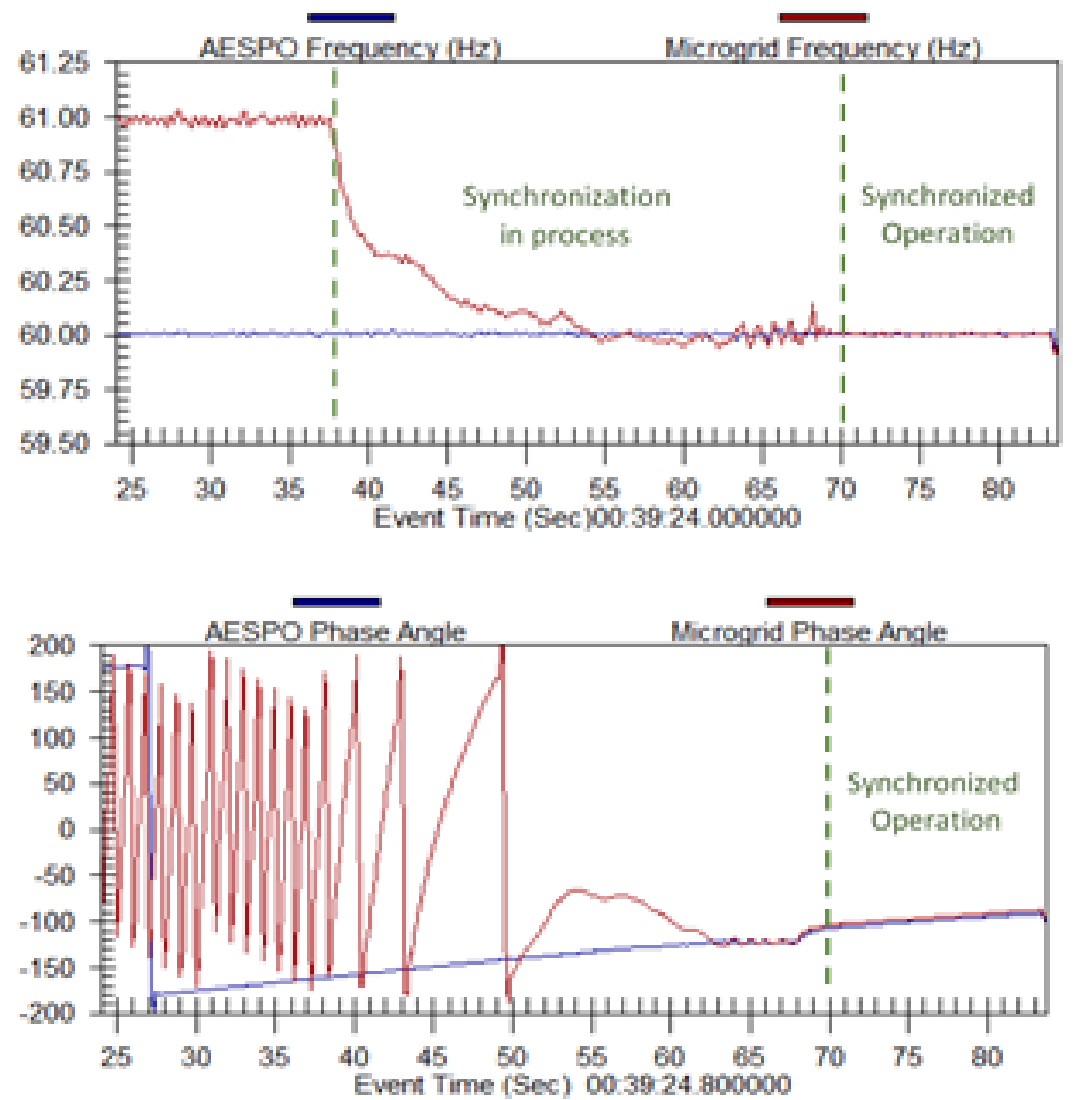

Figure 7.10: Tertiary control process. 
At the $70^{\text {th }}$ second, the AEPSO and microgrid frequency match, thus the synchronizer switch is closed. At the $76^{\text {th }}$ second, the applied torque to the generator shaft is increased to deliver more power to the system. Figure 7.10 (b) shows the phase angle difference between AEPSO and microgrid bus voltages. As synchronization occurs at the $70^{\text {th }}$ second, the phase angle difference decreases to a value almost equal to zero. This shows that the microgrid is synchronized to the host grid.

\subsubsection{Secondary Control (Islanding and AGC) Case Study}

Selecting one of the DER units to enable secondary control to restore the frequency to nominal value is a common practice in islanded operation. In this case, when overloading subsides and the microgrid starts to draw power from the host grid, AEPSO sends an ancillary service suspension request to the utility connected microgrid. Upon receiving request, microgrid gets disconnected from the host grid. The microgrid operator agent requests DER operator agents to serve as frequency regulator unit to restore the system frequency to nominal level by activating $A G C$ based secondary control behavior. DERs submit their interest with proposal messages along with the generation costs at that specific time according to cost functions in equations (7.1) and (7.2). In this case, inverter-based DER is qualified to enable AGC to restore the system frequency. Since prior to separation, the microgrid was importing power from the host grid, during the islanding situation, an immediate microgrid frequency dip is detected due to the power imbalance.

The islanding detection behavior is defined as IEC 61850 FRPRQ node which inherits from PTOF frequency protection logical node. A consecutive islanding detection algorithm is used to enable islanding detection which senses under/over frequency setting initially, then the frequency gradient is compared to set value. When islanding is detected, 
the microgrid operator enables DER operators to switch operation to droop control to enable accurate power sharing. Droop based primary control deviates the frequency from the nominal value according to the system loading conditions. AGC based secondary control is used to restore system frequency to nominal value.
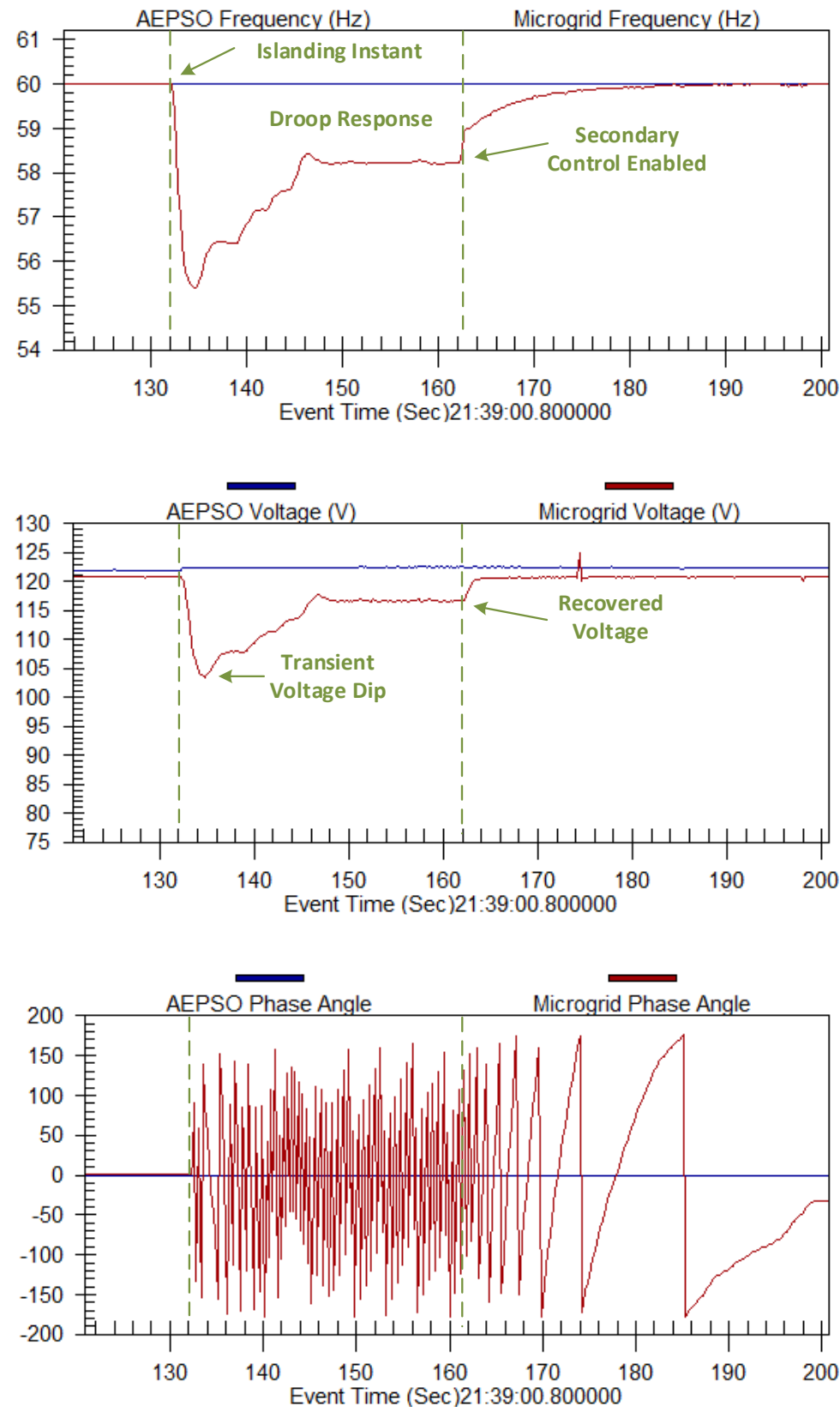

Figure 7.11: Secondary control process. 
Figure 7.11 (a)-(b)-(c) shows frequency, voltage and phase angle difference of AEPSO and microgrid during the secondary control process. From $100^{\text {th }}$ to $130^{\text {th }}$ second, the microgrid is operating in grid connected mode.

When islanding detected at $130^{\text {th }}$ second, the droop controller of the DERs are enabled. This results settling of the operation frequency to $58.5 \mathrm{~Hz}$ for the remainder of the operation in this loading level. Voltage is also settle around $115 \mathrm{~V}$. At $170^{\text {th }}$ secondary control is enabled by inverter-based DER to restore system frequency and voltage to nominal value of $60 \mathrm{~Hz}$ and $120 \mathrm{~V}$, respectively. The phase angle difference clearly shows the AEPSO and microgrid are operating separately.

\subsubsection{Distributed Control Consensus-based Economic Dispatch Case Study}

In this case study, we have adopted a two-level distributed economic dispatch method from literature using the incremental cost consensus strategy to successfully demonstrate the distributed control capability of the proposed framework [130],[131]. Consensus algorithm is applied to multi-agent systems to reach an agreement through information exchange between neighboring agents in the network. For this case study, same DER cost functions as in equations (7.1) and (7.2) are used. The incremental costs of the DERs are defined as equation (7.6), where $r i$ is incremental cost of DER unit $i$ [132]:

$$
r_{i}=\frac{d F_{i}\left(P_{i}\right)}{d P_{i}}=2 a_{i} P_{i}+b_{i}
$$

In this work, we neglected the generation capacity constraints and transmission losses for simplicity. While $P_{D}$ is the total power demand, when the incremental cost reaches equality, the incremental cost of common optimal point $r_{\text {opt }}$ becomes as (8) [133]:

$$
r_{o p t}=\left[\sum_{i=1}^{n} \frac{b_{i}}{2 a_{i}}+P_{D}\right] /\left[\sum_{i=1}^{n} \frac{1}{2 a_{i}}\right]
$$


The meshed microgrid network was constructed as shown in Figure.7.12.

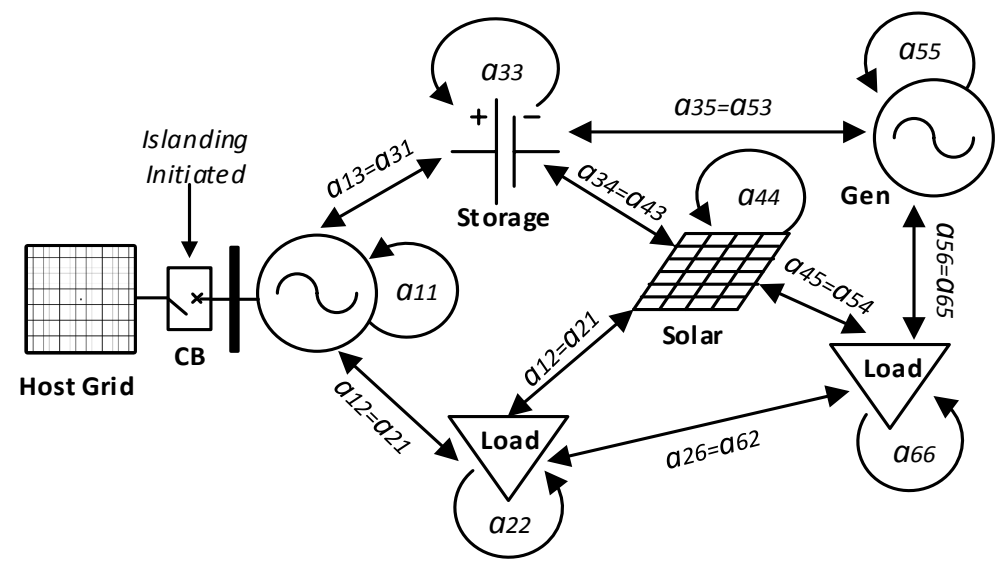

Figure 7.12: Graph representation of the microgrid network.

The network is a dynamic undirected graph $G=(V(t), E(t))$ with the set of nodes $V(t)=\{1,2, \ldots, n\}$ and edges $E(t) \subseteq V(t) x V(\mathrm{t})$. The neighbors of the agent $i$ are denoted by $\mathrm{N}(t)=\{j \in V(t):(i, j) \in E\}$. The set of edges, and set of neighbors of every agent in a dynamic graph is time-varying. Diagonal matrix is $D i, j:=\{\operatorname{deg}(v i)$, if $i=$ $j ; 0$ otherwise $\}$ contains the degree information of each vertex. The adjacency matrix $A=$ $[a i j] \in \mathbb{R}^{n x n}$ represents the nonnegative weights as equation (7.11) that any node $i$ conveys to communications received from node $j$, granted $j \in N i$ otherwise $a_{i j}=0$. A linear system in an undirected graph $(a i j=$ aji for all $i, j)$.

$$
\cdot\left|\begin{array}{cccccc}
1 & 1 & 1 & 0 & 0 & 0 \\
1 & 1 & 0 & 1 & 0 & 1 \\
1 & 0 & 1 & 1 & 1 & 0 \\
0 & 1 & 1 & 1 & 0 & 1 \\
0 & 0 & 1 & 0 & 1 & 1 \\
0 & 1 & 0 & 1 & 1 & 1
\end{array}\right| \stackrel{\text { yields }}{\longrightarrow}\left|\begin{array}{cccccc}
0.50 & 0.25 & 0.25 & 0 & 0 & 0 \\
0.25 & 0.25 & 0 & 0.25 & 0 & 0.25 \\
0.25 & 0 & 0.25 & 0.25 & 0.25 & 0 \\
0 & 0.25 & 0.25 & 0.25 & 0 & 0.25 \\
0 & 0 & 0.25 & 0 & 0.50 & 0.25 \\
0 & 0.25 & 0 & 0.25 & 0.25 & 0.25
\end{array}\right|
$$


The representation of a consensus algorithm guarantees the convergence to a collective decision via distributed agent communication if the adjacent matrix is left stochastic. It yields sum of state of all nodes is an invariant quantity.

$$
X_{i}^{\prime}(t)=\sum_{j=N_{t}} a_{i j}\left(x_{j}(t)-x_{i}(t)\right)
$$

The Metropolis Rule is used for assigning weights, as it is doubly-stochastic (both row and column, or left and right, stochastic). It has been shown that it guarantees stability and adaptation to topology changes with a near-optimal performance. In other words, the weights from $i$ to $j$ depends on the maximum number of neighbors either node has, and self-weight brings the total of each row (and column) in $A$ to 1 .

$$
a_{i j}=\left\{\begin{array}{c}
\frac{1}{\max \left(n_{i}, n_{j}\right)}, i \in N_{j} \backslash\{j\} \\
1-\sum_{i \in N_{j} \backslash\{j\}} a_{i j}, i=j
\end{array}\right.
$$

Two levels of the economic dispatch consensus protocol run simultaneously. First level is the information sharing to estimate $P_{D}$ power mismatch in the microgrid. The second level is used to reach a consensus on where all incremental costs must be equal to Lagrange multiplier $r_{\text {opt }}$. The updating rules of neighboring agents coordination is:

$$
\begin{array}{r}
r_{i}(t+1)=\sum_{j=N_{t}} r_{j}(t)-\varepsilon P_{D, i}(t) \\
P_{i}(t+1)=\left[r_{i}(t+1)-b_{i}\right] / a_{i} \\
P_{D, i}^{\prime}(t)=P_{D, i}(t)+\left(P_{i}(t+1)-P_{i}(t)\right) \\
P_{D, i}(t+1)=\sum_{j=N_{t}} a_{i j} P_{D, j}^{\prime}(t)
\end{array}
$$

In this use case, only distributed DER agents' cooperate without any hierarchy. The microgrid shown in Figure 7.12, transitions from grid-connected operation to islanded 
operation mode unintentionally. During the transition, a power mismatch $P_{D}$ prevails inside the microgrid. Each DER implements instantaneous magnitude of active power measurement logical node LDO.PMMUX.W.instMag for reaching to a consensus for the instantaneous $P_{D}$ power mismatch in the microgrid as per equation (7.15). Economic dispatch behavior is implemented in JADE platform to converge a common optimal incremental cost of the DERs. Once the optimal points are determined (converged), the optimal power references are known by each DER agent.

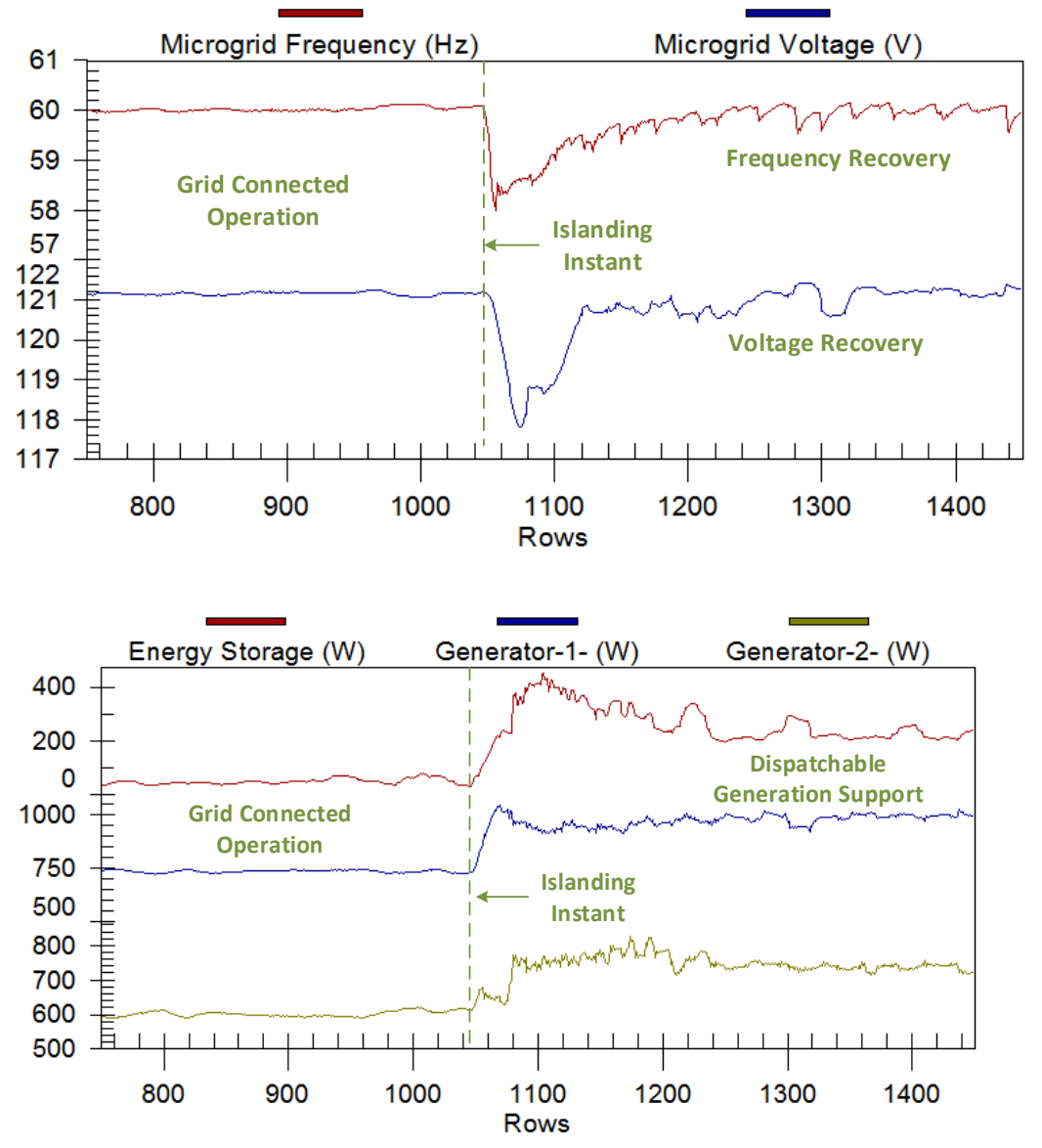

Figure 7.13: Distributed control results.

Accordingly, each DER agent adjusts its power generation. Figure 7.13 (a) illustrates the robustness of frequency and voltage recovery before and after islanding 
transition. Figure 7.13 (b) shows the power outputs of the DERs as per the distributed economic dispatch algorithm solution.

\subsection{Conclusion and Summary of the Results}

In this chapter, we presented a comprehensive testbed and an overview of development and application of real-world multiagent systems for decentralized and distributed control in smart grids. Behavioral modeling of the autonomous agents was presented and interoperability issues were discussed along with the standard data protocols. A state-of-the-art testbed was introduced to demonstrate the capabilities of the proposed multiagent testbed framework with case studies on decentralized hierarchical and distributed microgrid control. It has been shown that the proposed framework can be easily scaled and reconfigured for all smart grid domains. 


\section{DEVELOPMENT OF CLOUD COMMUNICATION FOR REMOTE ACCESS SMART GRID TESTBEDS}

\subsection{Introduction}

In this chapter, we present the development of a cloud communication to exploit remote access and smooth integration of smart grid testbeds. The developed cloud communication framework is integrated with previously developed cyber-physical power system infrastructure at Florida International University Smart Grid Testbed. In particular, in this chapter, a substation model is used to demonstrate the remote access platform along with the industrial generators, PMU, IED and PLC. Industrial data sets, models and metrics are presented. A realistic overcurrent protection experiment is demonstrated, as well as laboratory based information models.

The highly complex and interdisciplinary content of smart grid concept necessitates the integration of the testbeds with different capabilities for larger scale and extensive experimental verifications. The smart grid concept embraces extensive areas of interest, such as demand side management, market design, distribution automation, wide-area monitoring, protection and control. Testbeds are mainly built for particular project evaluation and verification purposes, yet do not provide a complete hardware/software test platform for comprehensive applications, such as renewable integration and cyber-security at the same time. With the advent of integrated remote access testbeds, extensive experimental platforms can be developed. Collaboration of the testbeds, which are built for 
various smart grid test platforms, can serve a special role in bringing together diverse experimental opportunities [134]-[135].

Although there is great interest in the application of cloud services on smart grids, few works have been reported in current literature. It is obvious that cloud communication will be one of the primary methods of communication in future power grid systems. Current research problems related to cloud-based energy management, information handling and security topics were addressed in [169]-[170]. Future opportunities, including home energy management (HEM), demand-side management (DSM) and building energy management (BEMS), were highlighted in comprehensive survey studies. Seamless Internet technologies that enable active interaction of power system utilities (providers) and intelligent buildings and microgrids (prosumers) were discussed in [171]. A tremendous amount of information handling compels the use of cloud-based mass data analyzer tools to tackle dynamic demand response problems in intelligent demand-side management and peak load relieve in smart grids. Machine learning models can be trained using cloudenabled massive datasets for optimization and planning purposes [172].

Most of the literature work has been done for consumer and producer-side managements. With the realization of cloud communication benefits, power system control and operation practices were proposed. Real-time information retrieval and ubiquitous access would improve the practices in smart transmission and distribution network operations [173]. PMU-based accurate tracking of the power grid enables improved stateestimation techniques. In [174], a cloud-based state estimation approach was proposed by synchronized phasor measurements from wide-scale deployed PMU devices. In order to provide robustness and avoid load balancing issues, the cloud-enabled power flow analysis 
method was proposed [175]. In [176], cloud computing applications were used to perform contingency analysis in cooperation with power flow studies. Optimization tools can run concurrently to enable optimization in smart grids [177]. This study addresses hardwarein-the-loop control challenges of cloud communication to advance smart grid controls. With this motivation, this chapter presents a state-of-the-art smart grid test bed platform using cloud communication interface to enable remote access.

Moreover, testbeds provide unique educational platforms for students as well as researchers for multi-user experiment facilities and proof of concept verifications [136]. Real-time testbeds are introduced for renewable integration at National Renewable Energy Laboratory (NREL) and cyber-security research at Idaho National Laboratory (INL). The integration of the testbeds would reduce the investment and labor costs in ongoing operational maintenance. The fast verification of concepts would spur research results to transfer into industry and broader public use at a faster pace. Smart grid concepts and developments can be verified through remote access by students without the ability to physically experiment on test beds. Testbed networks along large geographic distances would allow students and researchers to conveniently perform remote smart grid experiments. As a new generation of networked applications emerge, a diverse solution for large deployments of smart devices becomes possible. Cloud based communication systems provide solutions to embedded systems in the Internet of Things (IoT) [137]. In cloud-based systems, operational costs are significantly reduced compared to dedicated high bandwidth wide area links, which was previously a prerequisite for creating successful networking test beds. 


\subsection{Cloud Communication for Smart Grids}

Real-time on-demand data availability is crucial to enable interoperability of highly dispersed systems, such as power grids. Cloud-based services provide near to real-time information exchange using the Internet backbone.

\subsubsection{Cloud Service Types}

Cloud computing provides three distinct types of services: (i) Platform as a service (PaaS); (ii) Service as a service (SaaS); and (iii) Infrastructure as a Service. Clouds can be categorized depending on the deployment models: (i) Private; (ii) public; and (iii) hybrid [138]. In this framework, we use a SaaS platform providing secure end-to-end networking for smart grid devices, such as IEDs and PMUs. This platform can be implemented on virtually any new or existing system at a low cost capital and provides a web-based humanmachine-interface (HMI) for remote access and supervisory control [139].

\subsubsection{Emerging Cloud-based Smart Grid Applications}

With the widespread utilization of PMUs, complicated practices in power system control are becoming easier yet more dependent on advanced communication and real-time monitoring. Wide area control, including primary, secondary and tertiary levels, intelligent islanding detection and load shedding and distributed energy resource control are some of the emerging time-synchronized power system practices. Ubiquitous online data availability would enhance the reliability and resiliency of the complex power system control practices.

\subsubsection{Security, Privacy, and Delay Challenges}

Several fundamental security problems have arisen during the remote real-time access due to exposure to attacks from the Internet. When a system allows a user to access 
the system remotely, it naturally creates an attack surface for malicious users to attempt to gain access. Entry into a power system control network would be from within the IT infrastructure. Attacks from the IT network are less likely, but some kinds of problems in the IT network could disrupt normal network data flow on the power system network. In order to prevent malicious insiders, the cloud interface installs an agent within the power system network that collects the network information and pushes it to cloud service data servers. Since this connection is outbound, from the plant to the Internet, there is no requirement for the power system network to open any inbound TCP ports, thus the network never exposes itself to an attack surface from the Internet.

Privacy is among the key concerns for power system operators and end-users using the cloud communication. For that purpose, data encryption, authorization and user authentication protections are provided. Even though cloud connection hubs should be secure-by-design architecture to support efforts to protect against insecure application program interfaces (APIs), ultimately the account administrators must ensure that their APIs are secure. In power systems, three distinct control levels exist according to control response speed and communication infrastructure requirements. The primary control level deals with frequency and voltage control of the generators. Immediate response time is required in this level. Secondary level control deals with the frequency and voltage error correction with update rates in seconds. Tertiary control can be assumed to establish economic dispatch and optimization of the generators. The update rate is in minutes for this control level. This communication framework features real-time data throughputs up to 50,000 data changes per second at speeds of just a few milliseconds over network latency. The cloud provides solutions easily for secondary and tertiary level controls. 


\subsection{Smart Grid Test Bed Implementation}

The proposed cloud enabled remote access smart grid test bed platform is at the developed cyber-physical smart grid testbed at Florida International University. In particular for this study, a substation platform was constructed to perform real-time cloud data acquisition and experiment demonstrations. As shown in Figure 8.1, the substation interface involves actual synchronous generators, inverter-based DERs, transmission line models, and controllable active and reactive loads.

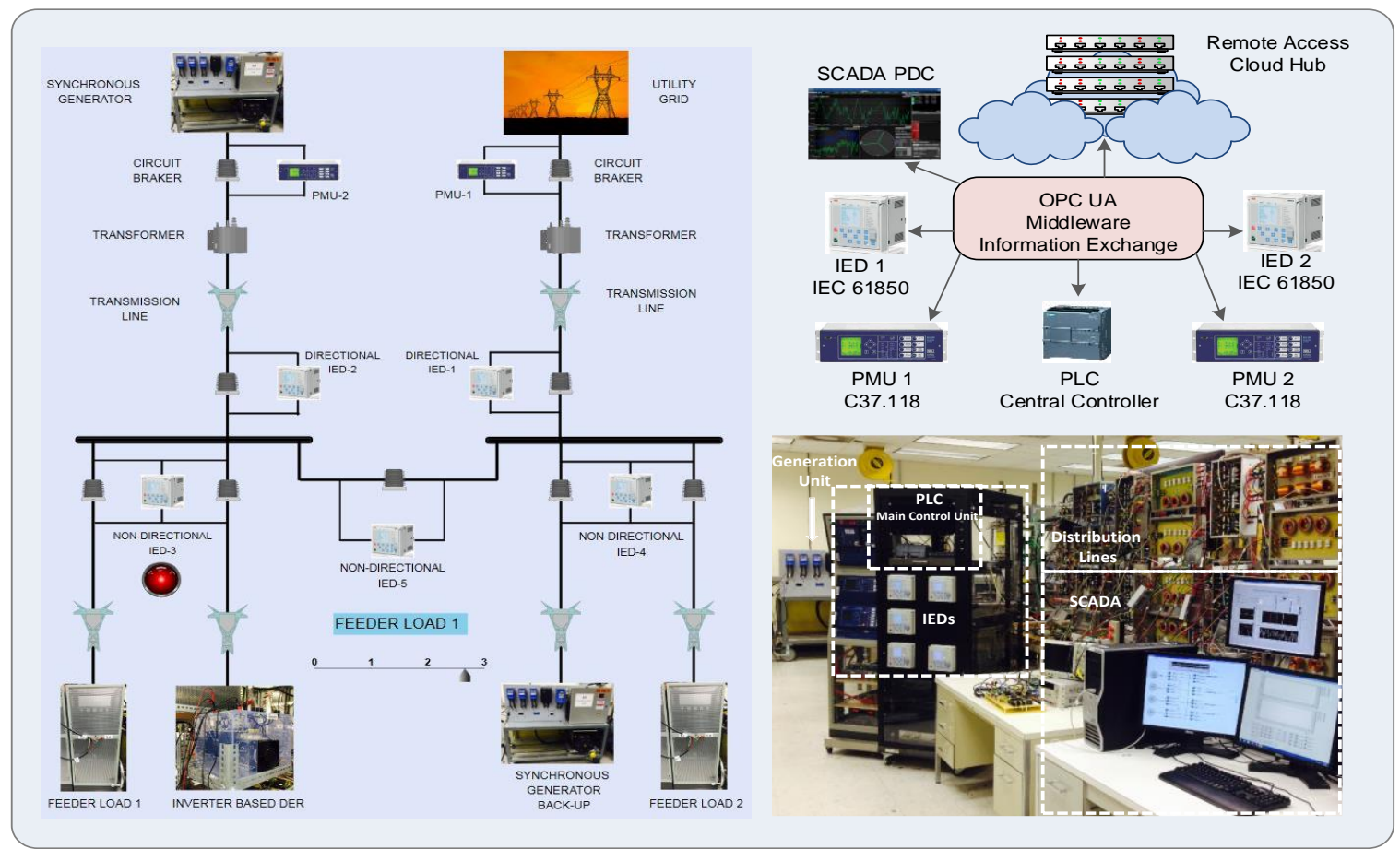

Figure 8.1: Cyber-physical infrastructure and cloud communication setup.

\subsubsection{Physical Hardware Assets}

The substation has two incoming feeders, two transformers, two main buses on the medium voltage side and four outgoing feeders. One of the incoming feeders is fed from the utility grid, while the other is connected to a synchronous generator. Incoming feeders are connected to bus bars through transmission line models. Two bus bars on the medium 
voltage side are connected through a bus transfer circuit breaker. Two of the outgoing feeders are connected to load models through transmission lines. One of the outgoing feeders is connected to an inverter-based DER, which is employed to model an integrated renewable generation unit. Another outgoing feeder is connected to a synchronous generator, which emulates a conventional generator, such as a diesel engine. The substation has protection schemes for functions, such as overcurrent, voltage and frequency. The bus bar is protected by overcurrent protection on incoming feeders.

\subsubsection{Cyber Information Domain}

Software using network tunneling was implemented to access data across the local Open Connectivity Unified Architecture (OPC UA) network. All OPC UA transactions are kept local to the computer, thus fully protecting the client programs from any network irregularities. The data across the network is mirrored, so that both server and client maintain a complete set of all the data. Remote access is enabled by polling/pushing OPC UA data sets from the cloud server. All data points are accessible remotely from cloud hub.

The substation includes two PMUs on the high voltage side of the incoming feeders. Two PMUs continuously report to a phasor data concentrator (PDC) on a central computer, which is located on the substation host machine. PMUs are deployed to provide wide-area situational awareness by displaying the disruptions that arise over a large geographic area. Aimed transmission points can be observed by time-synchronized collected data from PMUs with the PDC. A PDC is implemented as a software-based communication station, where PMUs provide time-synchronized measurements. The collected information is sorted according to time-stamp values. This enables a comparable real-time monitoring infrastructure with a high precision sampling. The collected data can be stored in a database 
system for future analysis or can be published to cloud communication media for remote real-time usage in remote stations. The IEEE Std. C37.118.1 and C37.118.2 define synchrophasor measurements and data transfer for power systems, respectively. In this work, Ethernet is used as lower-level communication protocol. A PMU supports high reporting rates, which can go up to 60 frames per second.

Directional protection IEDs are deployed on the medium voltage side of the incoming feeders. Each of the outgoing feeders and bus transfer mechanisms are equipped with non-directional protection IEDs. The IEDs involve protection schemes for functions, such as overcurrent, voltage and frequency. IEDs utilize IEC 61850 communication protocol. A PLC provides actuator capability using the Modbus protocol, which is implemented to control the load emulator and generator governor/excitation systems.

Modbus is the most popular open industrial protocol widely used due to its simplicity and ease of use. Numerous PLCs deployed in industry use the Modbus protocol. It uses master-slave communication, where the master sends a message that includes the targeted slave device address. All the devices in the network receive the message, however, only the device with the correct address responds.

The most common Modbus versions are ASCII, RTU and TCP/IP. Off-the-shelf OPC UA drivers are ubiquitous solutions for an easy implementation of interoperability frameworks. In this study, we deployed various OPC UA drivers in order to establish a common information exchange platform from industrial equipment, which uses different communication protocols. One of the OPC UA drivers is used to map PDC measurements to the network. Another OPC UA driver provides Modbus protocol mapping for the PLC and IEC 61850 for medium voltage protection IEDs. The driver provides solicited data 
access through IEC $61850 \mathrm{MMS}$ read and write requests and unsolicited data through MMS information reports. Accordingly, all of the shared information is available on any OPC UA Client.

\subsection{Experimental Demonstrations}

A remote load control, overcurrent protection, circuit breaker tripping and remote measurement study was demonstrated. Utility grid, two synchronous generators and an inverter-based (DER) are synchronized in the substation.

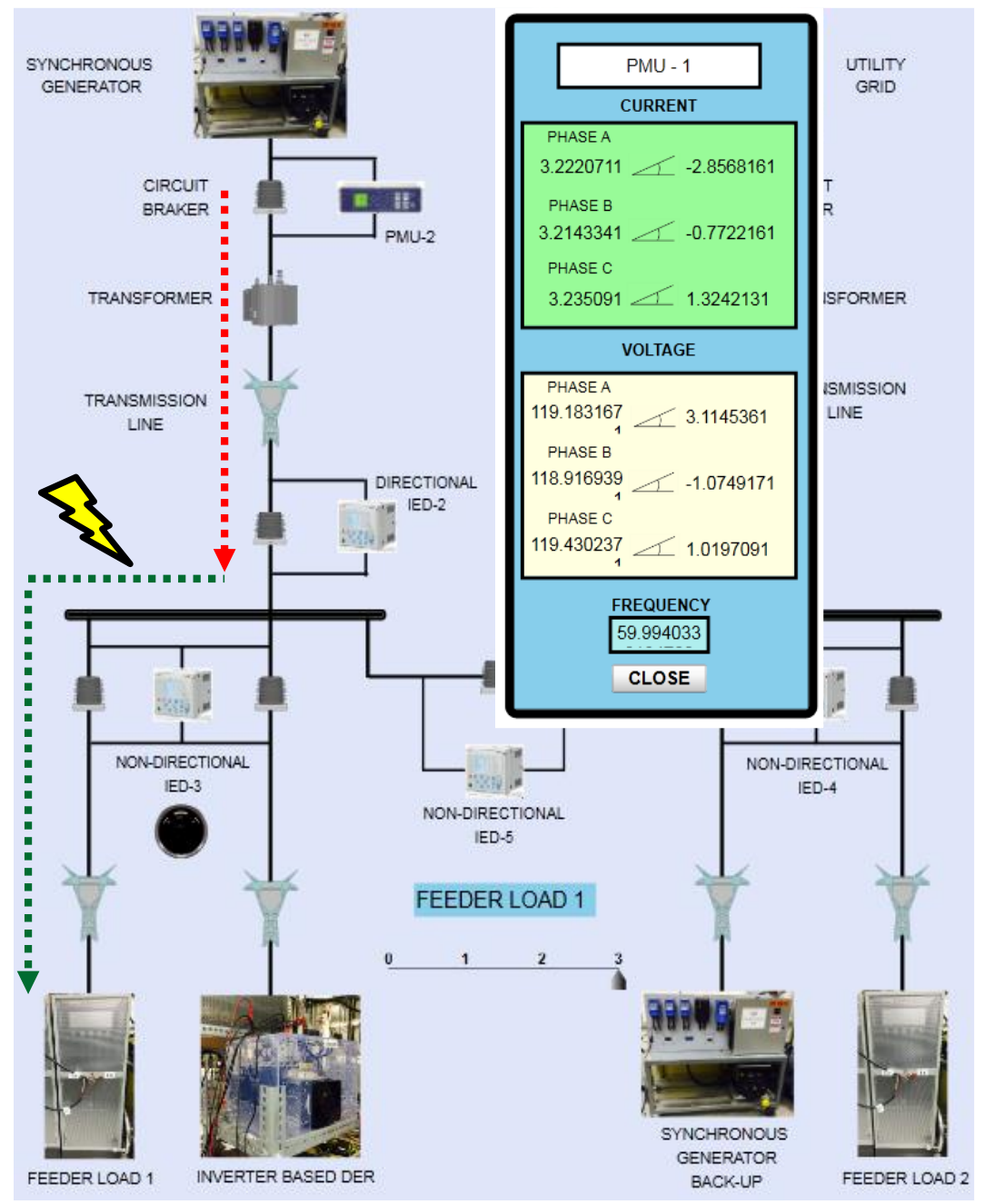

Figure 8.2: Overcurrent protection one line diagram. 
The feeder load illustrated in Figure 8.2 is protected by a non-directional protection IED-3. In order to demonstrate the overloading and overcurrent protection, the feeder load was increased gradually. Once the loading reached the threshold value, the circuit breaker tripped and the feeder 1 load was isolated. Figure 8.2 illustrates the substation measurements obtained from PMUs during the overcurrent protection.

Figure 8.3 shows the PDC SCADA interface including two PMUs on incoming feeders. 451 PMU and 421 PMU are connected to the utility source and synchronous generator, respectively. The figure includes frequency, voltage magnitude, three-phase angle difference, current magnitude, rate of change of voltage amplitude, single-phase voltage angle difference, active power, reactive power, and single-phase current angle difference measurements. The real-time experimental data is also saved for future analysis using various data formats, such as Common format for transient Data Exchange for power systems (COMTRADE) and American Standard Code for Information Interchange (ASCII) binary. This data formats store the oscillography and status data related to transient power disturbances related to the experiments.

In this specific experiment, the feeder load was increased gradually in steps to observe and create experimental event data for future analysis. As shown in Figure 8.3, at 4:22:05 PM, the overcurrent protection tripped the circuit breaker. The first figure shows the frequency oscillation of the system in response to high load tripping, and the second figure illustrates the voltage magnitude variation. The frequency jumped to $60.216 \mathrm{~Hz}$ and the voltage magnitude reached to $121.46 \mathrm{~V}$ momentarily. The transient voltage phasor displacement was observed. 


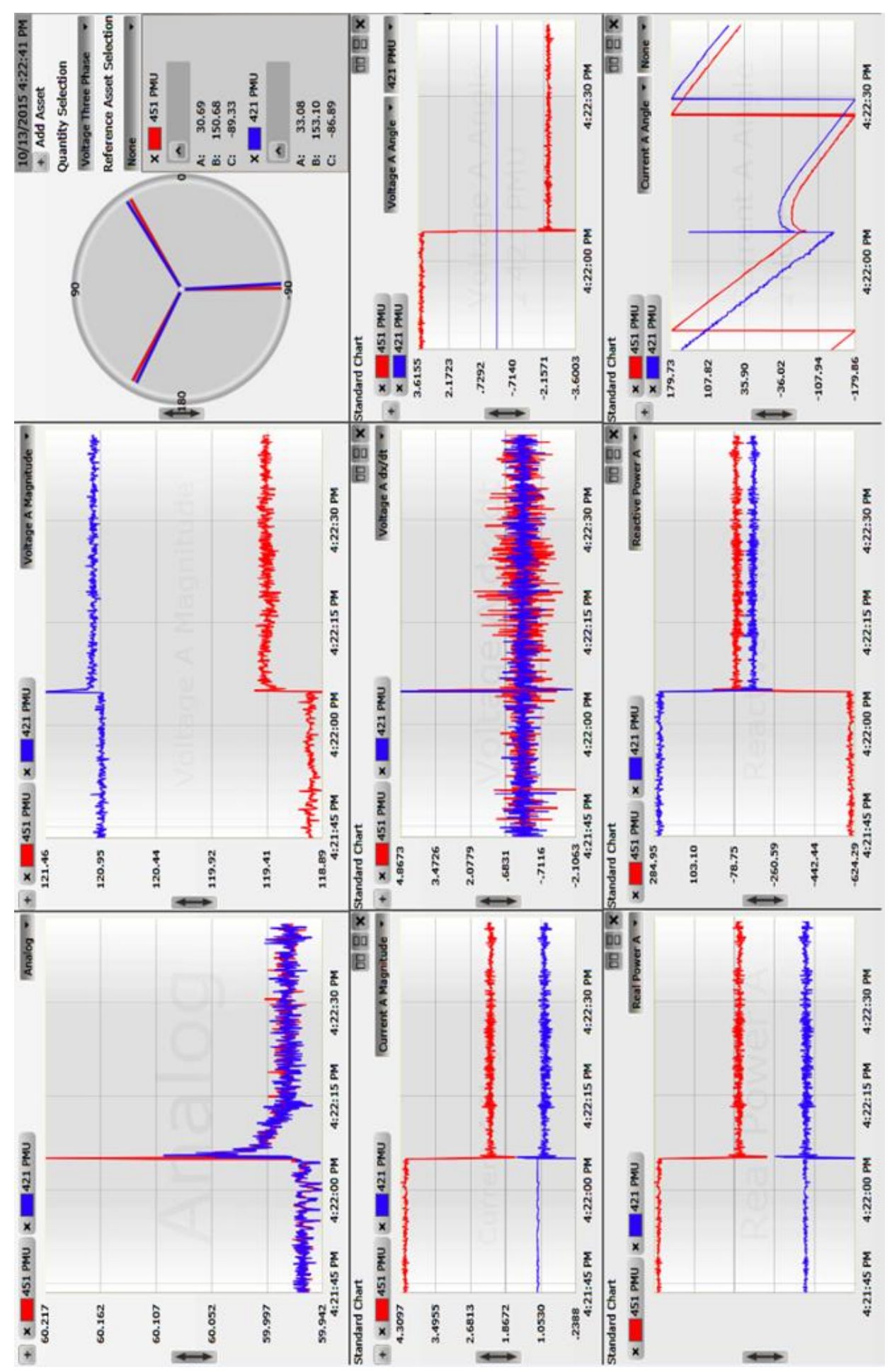

Figure 8.3: Overcurrent protection experimental results. 
The synchronous generator was operating at fixed active power, therefore after load tripping, its current and real power values remained the same. However, due to the load tripping, the received active power was reduced from the utility grid. The utility and synchronous generator continued to feed feeder load 2. During an overcurrent, the rate-ofchange-of-voltage (dx/dt) jumped instantly. The deviation was measured as $4.86 \mathrm{~V}$ per second. Voltage and current phase angle differences were changed according to the new system topology after the fault clearance. Since the synchronous generator was equipped with an automatic voltage regulator, reactive power injection was decreased due to less loading as result of load tripping.

This experiment provided a simple case study to demonstrate the capability of the various studies remotely. There are further experiments can be realized using the developed framework, such as generator tripping, load-shedding, islanding scenario, various protection applications.

\subsection{Summary of the Results and Conclusion}

In this chapter, a cloud-enabled architecture for remote access smart grid test beds was discussed. The proposed cloud communication mechanism was applied to a laboratory-based substation power system. An overcurrent protection scheme was illustrated as a demonstration case study. It has been presented that cloud communication can be successfully implemented for actual smart grid power system test beds. 


\section{MULTIAGENT-BASED FULLY DISTRUBTED MICROGRID CONTROL AND COMMUNICATION ARCHITECTURE}

\subsection{Introduction}

This chapter presents a multiagent-based fully distributed microgrid control and communication network design to support distributed control/optimization in smart grids. In this chapter, we investigate distributed optimization using a new fully distributed optimization method using diffusion strategy with a hierarchical approach to maintain frequency regulation and economic dispatch. The proposed control scheme employs peerto-peer communication among the agents replacing the need for a centralized controller to maintain system frequency regulation and additionally performs optimal economic dispatch simultaneously. The results are compared with centralized and consensus-based optimization algorithms. We have concluded that the proposed algorithm is superior over consensus algorithms in terms of convergence speed and utilizes reduced communication infrastructure compared to centralized controllers. Simulation demonstrations were conducted along with experimental results from a hardware-based microgrid using an industrial multiagent framework. The simulation and experimental results show that the proposed method and the agent framework can be deployed in real-world microgrids and offer superior decision making on optimal microgrid control.

Although distributed optimization and control solutions are evolving rapidly in the literature, there is a lack of agreement on communication infrastructure and networks for realistic implementations of distributed optimization in smart grids. The traditional electric 
service mechanism is undergoing continuous changes. Investigations are underway on operating the existing distribution network as dispersed microgrids. In future smart grids, the optimization infrastructure is expected to be restructured as a cyber-physical system, including smart devices, not only to carry power flow, but also to transmit data for protection via wireless and power line communications. Distributed control architectures inherently rely on tight coupling between the cyber and physical layers; therefore, the networked information systems are the core components of microgrids.

In a dynamic network, the implementation of a centralized control with access to all IEDs in the system is not realistic. Major drawbacks of the centralized methods are the requirement of high communication capability with a powerful central controller, and being susceptible to a single point failure. This can easily jeopardize the system with a complete collapse. Due to their inherent resilience, agent-based distributed approaches have the ability to self-check and react accordingly to the prevailing environmental conditions, even with communication constraints, where each agent can only communicate with its neighbors.

Wireless mesh networks with limited coverage would provide a reliable solution for internal microgrid communication with a peer-to-peer message exchange capability. Meanwhile, as a new generation of networked applications emerge, a diverse solution for large deployments of smart devices becomes possible through cloud based communication systems, providing embedded systems in the Internet of Things (IoT). In cloud-based systems, the operational costs are significantly reduced compared to dedicated high bandwidth wide area links which were previously prerequisites for creating successful 
networking of intelligent agents. In this chapter, we addressed two major problems that realistic multiagent-based distributed control schemes face:

- How to develop a communication network consisting of peer-to-peer communication infrastructure to achieve certain delay performance so that optimization convergence is guaranteed.

- How to develop optimization algorithm for individual microgrids implementing a fully distributed optimization algorithm featuring interconnected distributed energy resources.

\subsection{Wireless and Cloud Communication Architecture}

In this subsection, we intend to provide brief introduction for wireless and cloud communication infrastructure to send and receive power and load convergence values to and from neighboring DERs in a microgrid. Each agent acts as either a source or load, and the mesh network as a whole is considered as an individual, simulated microgrid. As each IED is considered an agent in the network, we attach them to sensors and loads to receive real time data and run an optimization algorithm in a distributed manner. The real-time information is transmitted through wireless mesh networks and cloud communication.

\subsubsection{Hybrid Wireless Mesh Protocol (IEEE 802.11s)}

IEEE 802.11s standard allows mesh networking among the intelligent electronic devices (IED) through 802.11 Medium Access Control (MAC) and Physical (PHY) layer standard. In our mesh network, the nodes in 802.11s WMN are given names based on their

roles. All nodes are considered as Mesh Points (MP) and are able to provide connectivity at the data link layer between other MPs. If an MP also provides connectivity to the Internet 
Cloud, it is termed a Mesh Portal Point (MPP). In our mesh network, the gateway collects IED readings that are sent by the IEDs via multi-hop routes. As wireless communication technologies leverage, they started taking over wired communication. However, wireless communication methods are not efficient to provide required area of reach expectations. As an amendment of IEEE 802.11n standard, IEEE 802.11s standard allows mesh networking on MAC/PHY layer.

The nodes in 802.11s Wireless Mesh Network (WMN) are given names based on their roles. All nodes are considered as Mesh Points (MP) and are able to provide connectivity at the data link layer between other MPs. If an MP also provides connectivity to the Internet, it is termed a Mesh Portal Point (MPP) and if MP is used as a wireless access point, it is called a Mesh Access Point (MAP). Devices that connect to these access points are called stations (STA). As IEEE 802.11s standard extends 802.11 MAC layer, routing is carried out in this layer rather than in the Network Layer. Because of this, routing is also called path selection for this standard. As most routing protocols are designed to work on Network Layer, new routing protocols are designed for 802.11s standard.

The QoS is an important aspect of wireless communication for efficient network usage. HWMP can sometimes suffer delay or bandwidth utilization problems. One method to evaluate QoS parameters in detecting end-to-end delay or remaining bandwidth in order to utilize the bandwidth and minimize the delay.

\subsubsection{Cloud Communication}

Real-time on-demand data availability is achieved using a cloud-based service providing near to real-time information exchange via Internet backbone. The SaaS platform is implemented to provide secure, end-to-end networking for microgrid IEDs. Cloud 
communication value is proven, and it is becoming a strong link in the industrial automation, smart grids, and variety of applications which require real-time on-demand data. In our developed cloud infrastructure, we adopted the software as a service (SaaS) mechanism which hosts software over a network, Internet in this case, for cloud computing, industrial Internet, machine-to-machine (M2M), and the Internet of Things (IoT) applications. SaaS means data collection, integration and distribution capabilities over a large span of network. This capability bring opportunities to handle big data driven smart grid applications and leverage the value of new or existing SCADA deployments. Industrial software as a service should provide as close to real-time performance as the network or Internet infrastructure will support. This means that cloud data updates are in milliseconds, not seconds or minutes. It can handle thousands of data changes per second, and support redundant connections.

\subsection{Multi-Agent System and Control Hierarchy}

This section introduces how the proposed multiagent scheme is integrated in the control hierarchy.

\subsubsection{Microgrid Control Hierarchy}

Three distinct control hierarchies are introduced according to the response speed and communication infrastructure requirements. Primary control adjusts the output power of each DER unit based on local measurements to maintain the system frequency and voltage. In general, depending on the control method (e.g. droop control), this control does not require distributed communication. This is because by definition primary control must act instantaneously and automatically, therefore it cannot be subject to communication 
delays. Secondary control adjusts the dispatchable assets shortly after frequency and voltage deviations to restore the system to nominal operating conditions. Tertiary control tracks the minimum global operating costs as DER costs fluctuate according to their output power, and state of charge in the case of energy storage systems. It is important to note that this hierarchy does not depend on a central entity, rather the control hierarchy is implemented within the agents themselves.

\subsubsection{Multiagent Framework}

In this chapter, as illustrated in Figure 9.1, we adopted a microgrid consisting of 9 nodes including a utility grid, two conventional dispatchable synchronous generators, one battery storage, two stochastic (non-dispatchable) wind turbine and photovoltaic generation units, one industrial, one commercial and one residential load. The various load nodes possess different consumption profiles. Each node is assigned to a corresponding intelligent agent that controls the power generated/consumed and is able to communicate only with neighboring agents.

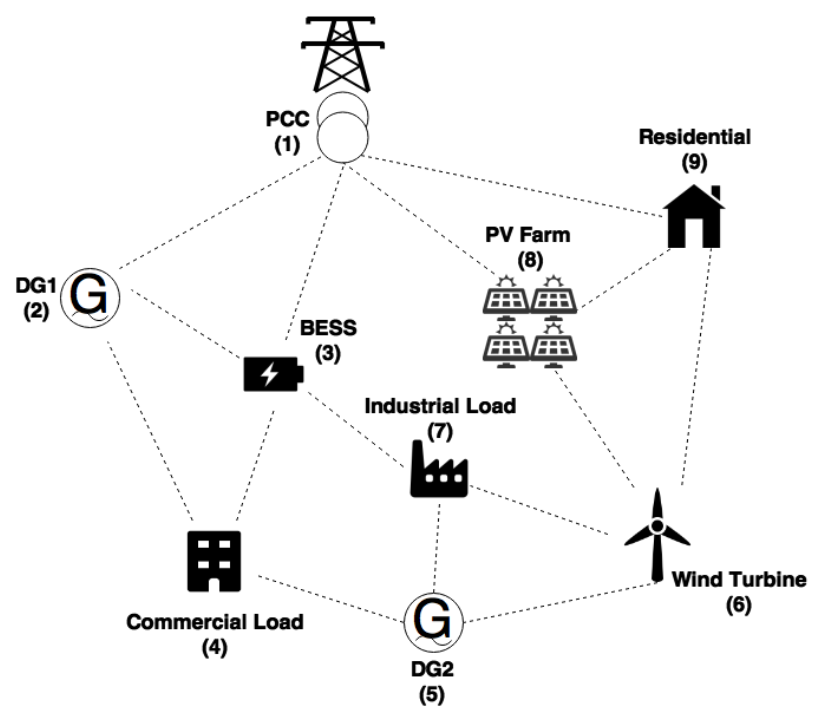

Figure 9.1 Microgrid nodes. 
The optimization process aims to minimize the total cost of the generation while maintaining the frequency regulation in the microgrid. Figure 9.2 shows the communication and agent layers of the microgrid. The device layer is situated on the lowest layer of the agent platform, where the physical electrical connections components are located.

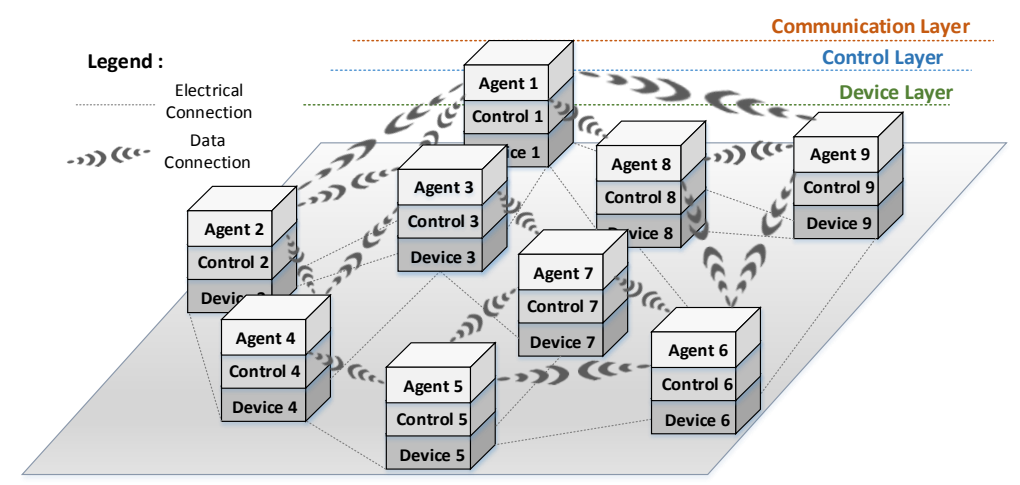

Figure 9.2 Agent communication network.

The primary control is located in the middle layer directly controlling the power output of the corresponding devices. This layer mainly deals with the power being drawn or delivered for the load and stochastic generation units. Droop control is implemented in this layer for conventional synchronous generators and battery storage systems as the primary frequency control. The top level termed the agent and communication layer implements the diffusion strategy to provide secondary and tertiary control. Thus, the agent layer substitutes the centralized microgrid control structure.

Load agents and stochastic DER agents extract the power measurements from the device layer and diffuse the information to neighboring agents. The Point of Common Coupling (PCC) of the utility grid measures the delivered and drawn power of the microgrid and controls the islanding decision. For the dispatchable DERs, the agent layer operates to adjust the power levels of each unit in two separate time scales: (i) As secondary 
response, to adjust online adaptive no-load frequency shortly after the primary response;

(ii) As tertiary response, gradually and continuously tracking the global optimal economic dispatch solution. The resulting operating points are informed through the control layer to the DER actuators located on the device layer, which adjust the corresponding device power output.

\subsubsection{Agent Descriptions}

Five types of agents are defined in the microgrid context. The cost and the marginal cost functions are used in the distributed optimization formulation.

Load Agents: They correspond to the industrial, commercial and residential load models. The consumed power cannot be precisely predicted as a result of stochastic consumer behavior. They measure and broadcast the instantaneous power requirements of their connected loads.

Renewable DER Agents: This agent type is associated with stochastic (nondispatchable) wind and solar DERs. Similar to load agents, the generation profiles cannot be accurately forecasted, therefore they are counted as negative loads. They measure and broadcast the instantaneous generated power.

Conventional DER Agents: This agent type is associated with dispatchable synchronous generators such as diesel or natural gas plants. They possess an ability to manage their output power. Each DER's corresponding cost function is not known by other agents. They broadcast their current marginal cost according to the typical quadratic cost functions:

$$
\begin{aligned}
F_{D G} & =a+b P_{D G}+c P_{D G}^{2} \\
\frac{d F_{D G}}{d P_{D G}} & =b+2 c P_{D G}
\end{aligned}
$$




$$
P_{D G, \min } \leq P_{D G} \leq P_{D G, \max }
$$

where $F_{D G}$ is the quadratic cost function for a fossil fuel distributed generator (DG). Constants $a, b$, and $c$ are the quadratic coefficients, $P_{D G}$ is the power level for the unit. The derivative of the cost function with respect to power is $d F_{D G} / d P_{D G}$, which is also known as the marginal cost function. The marginal cost function is used by the optimization diffusion algorithm to reach the economical dispatch point of DERs.

Energy Storage Agents: The storage agent is also a dispatchable type, which has the capability to transfer power bidirectionally; either charging or discharging. The battery storage agents should ideally charge when the rate of electricity (or marginal cost) is low, and oppositely discharge when high. There is a lack of agreement of what the cost function of a storage system should be. In this chapter, a more general cost function is proposed that is technology-agnostic:

$$
\begin{gathered}
F_{B}=\mathrm{x}+\mathrm{y}\left(P_{B}+3 P_{B, \max }(1-S O C)\right)+z\left(P_{B}+3 P_{B, \max }(1-S O C)\right)^{2} \\
\frac{d F_{B}}{d P_{B}}=\mathrm{y}+\mathrm{z}\left(2 P_{B}-2 P_{B, \max }(S O C-1)\right) \\
-P_{B, \max } \leq P_{B} \leq P_{B, \max }
\end{gathered}
$$

where $F_{B}$ is the cost of the power, $P_{B}$, being charged or discharged from the storage, $P_{B, \max }$ is the maximum charge or discharge rate, SOC is the current state of charge, and $x$, $y$ and $z$ are the quadratic coefficients. The marginal cost $d F_{B} / d P_{B}$ is proportional to the power drawn and inversely proportional to the SOC, meaning the marginal cost will be lower as SOC approaches $100 \%$. The relationship between power, SOC and marginal cost is illustrated in Figure 9.3. 


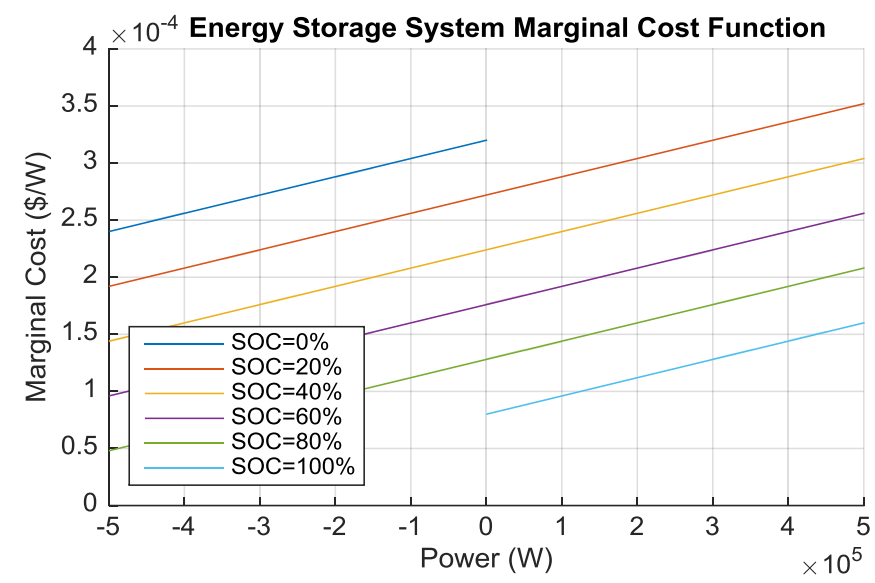

Figure 9.3 Energy storage system marginal cost function.

This cost function is formulated in such a way that the energy storage systems automatically charge when the microgrid's marginal cost is low, and discharge when it is high. In this way, the storage asset automatically performs in a cost effective way by maximizing the energy arbitrage potential with the distributed optimization signal.

Utility Grid Agent: This agent is active when the microgrid is operating in grid-tied mode. The utility grid agent is of a dispatchable type. It monitors the delivered and received power to the microgrid. It broadcasts the current electricity rate being offered by the utility, which can be assumed as a constant marginal cost.

$$
\begin{aligned}
& F_{G}=r_{u} \cdot P_{G} \\
& \frac{d F_{G}}{d P_{G}}=r_{u}
\end{aligned}
$$

where $F_{G}$ is the cost of the power, $P_{G}$ is the power delivered or received and $r_{u}$ is the current electric rate being charged or offered by the utility grid. Thus, the cost is a linear function of the power, and the marginal cost $d F_{G} / d P_{G}$ is a scalar value equal to electric rate.

There are two main modes of operation; islanded and grid-tied mode. In the former, the optimal (economic dispatch) operation of the dispatchable agents is at the point where 
every asset's marginal cost function is equal to the others while maintaining the net power demand. This is known as the Lagrangian point $(\lambda)$. When grid-tied, all the dispatchable agents converge to the point where all marginal costs are equal to the electric rate given as a constant marginal cost.

$$
\frac{d F_{1}}{d P_{1}}=\frac{d F_{2}}{d P_{2}}=\cdots=\frac{d F_{n}}{d P_{n}}=\lambda(=) r_{u}
$$

\subsection{Diffusion Strategy and Implementation}

Traditionally, the distributed information sharing between neighboring agents is established through consensus-protocol. The diffusion strategy includes a stochastic gradient term to expedite the process and reach convergence much quicker. Additionally, by including the gradient of the cost function in the formulation, diffusion can reach the economic dispatch point through distributed optimization. In this study, as shown in Figure 9.4, two separate diffusion processes are implemented: (i) Information sharing diffusion; (ii) Optimization diffusion.

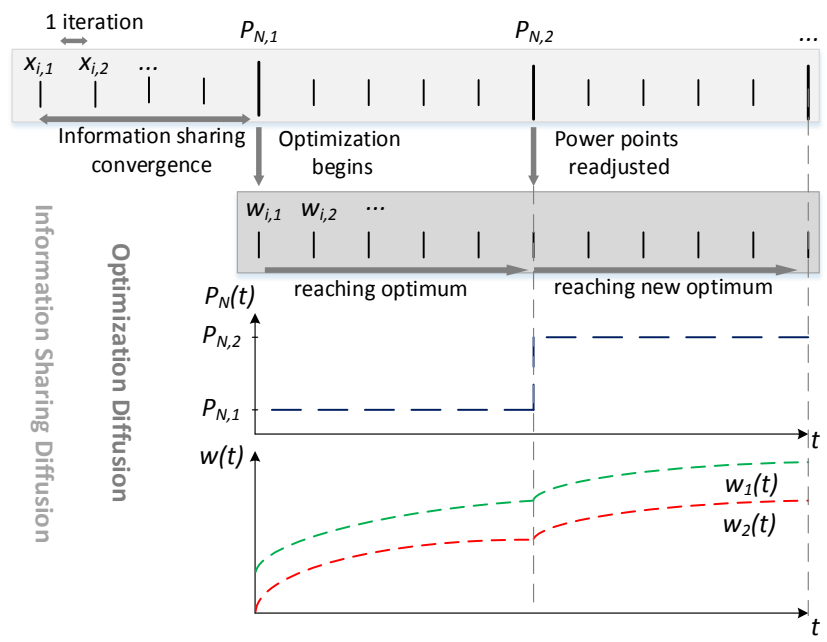

Figure 9.4 Diffusion strategy. 
The information sharing diffusion is formulated to broadcast the instantaneous stochastic power consumed by the load agents and generated by non-dispatchable renewable DER agents plus network losses. This process continuously updates the network's net power demand to be supplied by the dispatchable DER agents.

$$
P_{N}=\sum P_{L A}-\sum P_{R A}+\sum P_{\text {Loss }}
$$

Where $P_{N}$ is the net power demand, which is defined by the addition of all load agents $\left(P_{L A}\right)$ minus all intermittent renewable DER output $\left(P_{R A}\right)$ plus the network losses ( $\left.P_{\text {Loss }}\right)$. We assumed the resistance $R_{i j}$ of each distribution line is known. Thus, each agent calculates their local contribution to the network losses, and this value is included in the information sharing diffusion process as described in the next sub-section.

As shown in Figure 9.4, the information sharing process restarts after a certain precision level (acceptable residual convergence error) has been reached among all agents. Programmatically, this can be implemented with a system of flags that are communicated among neighboring agents to assure all agents in the network have reached convergence.

After the first convergence, the net power demand of the network is known, thus the economic dispatch is initiated. The optimization diffusion is used to adjust the dispatchable DERs to their economic dispatch point on a continuous basis as the optimal solution is approached after every iteration. When the information sharing converges once again, the economic dispatch points are shifted, and the optimization begins converging to the new optimal points. These two complementary processes continue indefinitely tracking the net power demand, and the economic dispatch solution. 


\subsubsection{Information Sharing Diffusion}

The communication between neighboring agents is bidirectional. The nonnegative communication coefficient from agent $i$ to agent $\mathrm{j}$ is defined as aij, with n number of agents. Coefficient aij can be assumed as a trust factor that each agent places on the other. The coefficients do not need to be symmetric, i.e. aij $\neq a j i$. They must satisfy the following conditions:

$$
a_{i j} \geq 0, \quad \sum_{i=1}^{n} a_{i j}=1, \quad \text { and } a_{i j}=0, \text { if } \mathrm{j} \nexists N_{i}
$$

where Ni represents the neighborhood, or a group of agents within communication reach, of agent $i$. As a condition for convergence and stability of the diffusion algorithm, the adjacency matrix of weights $A=[a i j] \in \mathbb{R} n * n$ must be a left-stochastic matrix satisfying $A T \mathbb{1}=\mathbb{1}$, where $\mathbb{1}$ is an $n^{*} 1$ vector of all ones.

$$
a_{i j}=\left[\begin{array}{l}
\frac{1}{\max \left(n_{i}, n_{j}\right)}, i \in N_{j} \backslash\{j\} \\
1-\sum_{i \in N_{j} \backslash\{j\}} a_{i j}=j
\end{array}\right.
$$

The Metropolis rule, equation 9.12 is applied to construct a symmetric and doubly stochastic (right and left) A matrix, where $n i$ is the number of connections out of the node $i$. Metropolis weights guarantee stability, good performance and the ability to adapt to changing topologies. Adaptability via automatic agent discovery is crucial for altering topologies as a result of incoming or outgoing agents in the network.

The Metropolis rule depends only on the local information available to each agent, and that of its neighbors. The adjacency matrix can be constructed on the fly through the number of neighbors each agent has. This is realized by assigning a unique ID to each node and communicating this ID, along with the iteration number, among the neighbors to create a local database of the global agent network. 
The diameter of the network, $\mathrm{d}$, is defined as the maximum shortest path from any node to another. After $\mathrm{d}$ iterations, all nodes achieve a global knowledge of the node population in the microgrid network. Likewise, if a node stops communicating, this node is dropped from the local database after d iterations, and the Metropolis weights are recalculated. In the real-time experimental study, we have implemented agents in the Java agent development framework (JADE). This framework supports automatic agent discovery by assigning a unique serial number for each agent in the network.

The Combine-Then-Adapt (CTA) diffusion strategy is implemented for information sharing. CTA employs a gradient term in an intermediate step which is disseminated to neigh-boring agents. This allows for information to "diffuse" more quickly beyond the neighborhood of any given agent:

$$
\text { CTA }_{\text {Diffusion }}=\left[\begin{array}{c}
\varphi_{i, k-1}=\sum_{j \in N_{i}} a_{i j} x_{j, k-1} \\
x_{i, k}=\varphi_{i, k-1}-\mu \hat{s}_{i, k}\left(\varphi_{i, k-1}\right)
\end{array}\right.
$$

Where $x_{i, k} \in \mathbb{R}$ denotes the state of agent $i$ at time $k, \phi_{i, k}$ is the intermediate variable for agent $i$ at time $k, \mu_{i}$ is a nonnegative updating parameter of agent $i$, and $\hat{s}_{i, k}\left(\varphi_{i, k-1}\right)$ is the stochastic gradient for agent $i$ of the intermediate state $\phi$ at time $k$. The stochastic gradient is simply calculated on the difference of $\phi$ from one iteration to the other; thus, each agents keeps track of its gradient and updates the combination of all its neighbors accordingly.

In our microgrid control scheme, equation (9.13) is used to broadcast $P_{N}$ to all agents in the network. Because the purpose of the information sharing is to know the net power demand, only the stochastic agents (load and renewable DER) communicate their current power status; dispatchable agents only communicate their contribution to the 
network losses. Then, $P_{N}$ is used for the distributed optimization of the diffusion strategy. To compare the performance of diffusion versus consensus, we take a hypothetical scenario using the microgrid shown in Figure 9.1. Suppose the commercial, industrial and residential loads were consuming $200 \mathrm{~kW}, 300 \mathrm{~kW}$ and $50 \mathrm{~kW}$, respectively; and the wind and solar farm were producing $100 \mathrm{~kW}$ each. Taking these as starting points for our convergence analysis (the dispatchable nodes being at zero), we get the performance shown in Figure 9.5. As observed in Figure 9.5, diffusion reaches the same level of precision in less than half the iterations needed for consensus (18 vs 39$)$. This is clearly a substantial improvement that can be used for more precise tracking of microgrid conditions for faster frequency response. The value reached, $-38.89 \mathrm{~kW}$ is the average of the net power requirement. When multiplied by the number of nodes $n$ in the network, $P_{N}$ is obtained: $38.89 k W * 9=-350 k W=100 k W+100 k W-200 k W-300 k W-50 k W$.
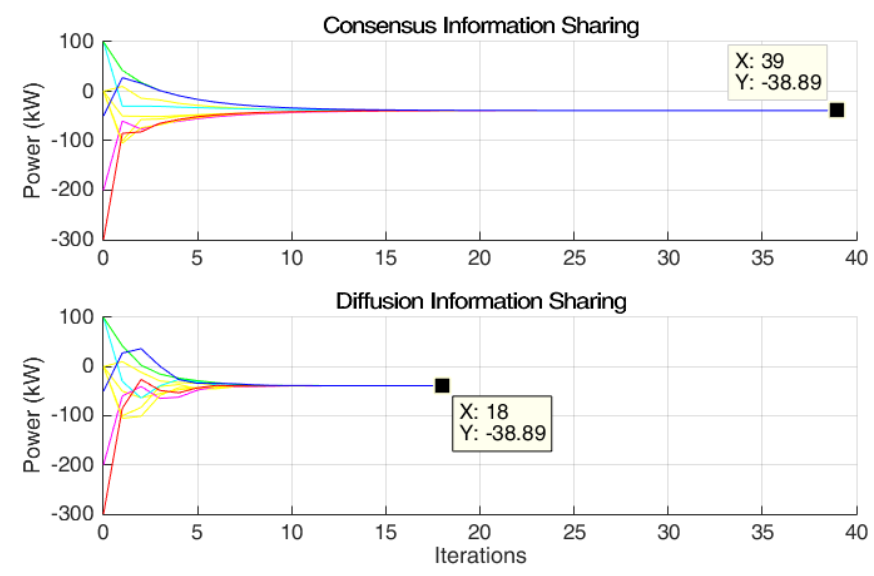

Figure 9.5 Consensus vs diffusion information sharing (9-Node).

Although for small scale systems a centralized method would outperform diffusion since no iterations are needed, the proposed method foregoes any communication infrastructure and is therefore valuable for any system that extends beyond one single agent. The scalability of the diffusion method is tested with the well-known IEEE 14-Note 
Test System and IEEE Reliability Test System-1996, which has 73 nodes. Table I shows the results of applying the proposed method to these layouts and comparing the performance of the diffusion algorithm versus consensus. The same Metropolis weights were used, taking the electrical connections as the edges of the graph, and the starting points were randomized within $\pm 400 \mathrm{~kW}$.

Table 9.1 Diffusion vs. consensus convergence comparison

\begin{tabular}{|c|c|c|c|}
\hline Test System & $\begin{array}{c}\text { Consensus } \\
\text { Iterations }\end{array}$ & Diffusion Iterations & $\begin{array}{c}\text { Diffusion Time to } \\
\text { Converge vs } \\
\text { Consensus }\end{array}$ \\
\hline 9-Node & 39 & 18 & $46 \%$ \\
\hline IEEE 14-Node & 106 & 34 & $32 \%$ \\
\hline $\begin{array}{c}\text { IEEE RTS-96 } \\
(73 \text { Node) }\end{array}$ & 736 & 103 & $14 \%$ \\
\hline
\end{tabular}

Figure 9.6 illustrates the significant performance improvement for the RTS-96 system from 736 iterations with consensus to 103 with diffusion. It is important to note that $\mu$ in equation 9.13 is a key parameter for the convergence of the information sharing diffusion and affects the performance of the algorithm.

In these test cases a trial-and-error method was used to select the most effective parameter for each case. Also, a 73-node network would be considered as a bulk-generation power system, and even in such a vast electrical structure the diffusion algorithm shows promise in distributed control.

With modern communication technologies, the iterations can actually run in the milliseconds, so even for the 73-node case, convergence could realistically be reached in a few seconds. As the number of dispatchable units' increases, the frequency regulation is also easier due to the increase in inertia and/or droop support. 

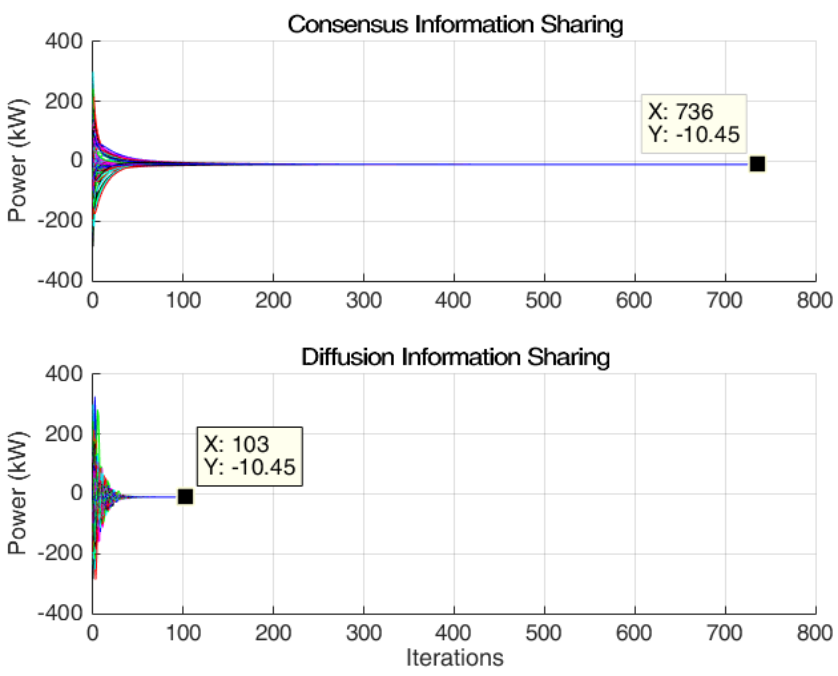

Figure 9.6 Consensus vs diffusion information sharing (73-Node).

\subsubsection{Optimization Diffusion}

The previous diffusion formulation includes a stochastic gradient in the information sharing (also known as estimation) problem. However, in optimization diffusion, the marginal cost functions of the DERs are used instead. First the global problem is defined:

$$
\begin{gathered}
J_{g l o b}(\mathrm{w})=\sum_{i=1}^{n} J_{i}(w) \\
\min _{w} J_{g l o b}(\mathrm{w})
\end{gathered}
$$

Where $J_{i}$ is the cost function of agent $i$ defined over the real-valued vector of arguments $w \in \mathbb{R}^{n}$, representing the power output of each dispatchable agent. Therefore, the objective is to minimize the global cost $J_{g l o b}$, which is the sum of all the individual cost functions. The cost functions do not need to be the same for each agent, as is the case for the microgrid where each agent has its own cost function.

All $J_{i}$ need to be convex and differentiable, and at least one $J$ needs to be strongly convex in order for $J_{g l o b}$ to also be strongly convex, meaning there is only one global minimum solution. Although assuming convex functions is a simplification of the DG cost 
functions due to the valve-point loading effect, multiple fuel options, and prohibited operating zones, non-convex distributed optimization is beyond the scope of this work.

In this chapter, the cost functions of the DERs are all strongly convex with the exception of the utility grid agent which is only convex (linear functions are both convex and concave). The conventional DER agents' and energy storage agents' cost functions are strongly convex since they are positive quadratic functions. Thus, $J_{g l o b}$ is also strongly convex and can reach a global minimum through optimization.

Similar to CTA diffusion for the estimation problem in equation 9.13, CTA can be used for distributed optimization by adopting the gradient of the cost function instead of the stochastic gradient:

$$
C T A_{\text {Optimization }}=\left[\begin{array}{c}
\varphi_{i, k-1}=\sum_{j \in N_{i}} a_{i j} w_{j, k-1} \\
w_{i, k}=\varphi_{i, k-1}-\mu \nabla_{w^{T}} J_{i}\left(\varphi_{i, k-1}\right)
\end{array}\right.
$$

Where $w_{i, k}$ is the $i^{\text {th }}$ agent's estimate of $w$ at time $k$, and $\nabla_{w^{T}} J_{i}\left(\varphi_{i, k-1}\right)$ is the gradient of agent $i$ 's cost function calculated with the intermediate variable $\phi_{i}$; all other parameters are the same as equation 9.13. The weights also remain the same Metropolis values as with information sharing.

During the optimization, not all agents necessarily need to be informed, or have an associated cost function. Only conventional DER agents, utility grid agents and energy storage agents have a gradient for the second term of equation 9.16. All other agents are uninformed. However, these uninformed agents still have a purpose in disseminating the information across the network by implementing the first term of equation 9.16.

The vector of arguments $w$ has an entry for each of the agents in the network, however only the dispatchable DERs have a nonzero value. All other nodes on the network 
remain at zero and are not updated. Since each agent has unique knowledge of its own resource, the cost function equation 9.17 and gradient equation 9.18 for dispatchable agent $i$ are only defined for the $i^{\text {th }}$ element of vector $w$. All other elements remain at zero.

$$
\begin{aligned}
& J_{i}\left(\mathrm{w}_{i}\right)=F_{i}\left(\mathrm{w}_{i}[i]\right) \\
& \nabla J_{i}\left(\mathrm{w}_{i}\right)=\nabla F_{i}\left(\mathrm{w}_{i}[i]\right)
\end{aligned}
$$

Thus, the gradient update step in the second term of equation 9.16 only affects the agent's own power level (if such a gradient exists). Through CTA optimization, each dispatchable agent's gradient term converges to the economic dispatch or Lagrangian point. However, an additional step in the updating procedure must be included for the microgrid optimization system. This is explained as the nudge methodology in the next section of modified optimization diffusion.

\subsubsection{Modified Optimization Diffusion}

The net power demand must be supplied among the dispatchable agents. This is where the information sharing and optimization diffusion methods are merged. The net power is included in the optimization formulation in the following way:

$$
\sum_{i=1}^{n} w[i]=P_{N}
$$

To keep the equality in equation 9.19, after the gradient update by the dispatchable agents in equation 9.16, a nudge by the agent being updated gets added to the other dispatchable agents plus any deficit compared to the net power:

$$
\begin{gathered}
\Delta w_{i, k}[i]=-\mu \nabla_{w^{T}} J_{i}\left(\varphi_{i, k-1}\right) \\
\sum_{j=1}^{n} \Delta w_{i, k}[j]=P_{N}-\sum w_{i, k-1}-\Delta w_{i, k}[i]
\end{gathered}
$$


where $\Delta w_{i, k}[i]$ is the so called "gradient nudge" of equation 9.16. Equation 9.21 and Figure 9.7 describe how the nudge is distributed among the other dispatchable agents to maintain the net power demand.

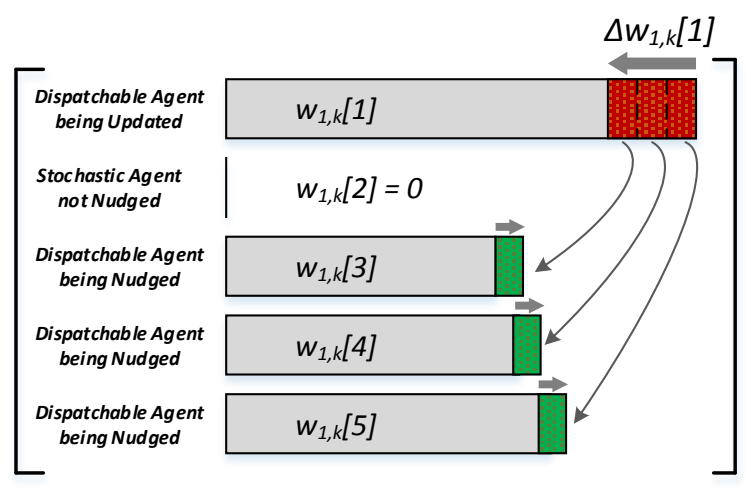

Figure 9.7 Nudge methodology.

Intuitively, if one of the DER's marginal cost is greater than the rest, by decreasing that particular unit's power level, and increasing the others by the same amount in a collective way, a lower total cost is reached since the former unit is "nudged down" in cost a greater amount than the others are "nudged up". Because the $w_{i}[i]$ of stochastic units always stays at zero, the dispatchable units will know which other nodes are also dispatchable to distribute the nudge to them. This is how the economic dispatch point is reached in a distributed way, and is the key variance of the proposed modified diffusion method in this study to other existing diffusion methods in the literature. The power levels would drift to each agent's minimum cost and the power balance would not be maintained unless this methodology is used.

Additional to the net power constraint, the dispatchable agents have unique constraints on the minimum and maximum power of their corresponding resource. A barrier or penalty function is included around the feasible region. The penalty method is preferred, as it 
maintains the continuity of the cost function and can converge to a solution even if the initial or intermediate states fall beyond the constraints. The objective described by equation 9.15 becomes:

$$
\delta_{i}\left(w_{i}\right)=\left[\begin{array}{cc}
\min _{w} J_{g l o b}(\mathrm{w})+\sigma \sum_{i=1}^{n} \delta_{i}\left(w_{i}\right) \\
0 & w_{i, \min } \leq w_{i} \leq w_{i, \max } \\
\alpha\left(w_{i}-w_{i, \max }\right)^{2} & w_{i}>w_{i, \max } \\
\alpha\left(w_{i}-w_{i, \min }\right)^{2} & w_{i}<w_{i, \min }
\end{array}\right.
$$

where $\sigma$ is a parameter which dictates the importance of obeying the constraints, and $\delta$ is the penalty function defined for each element in $w$ according to its inequality constraints. Equation 9.23 describes a simple addition to the cost function of the squared deviation from the constraint tuned by scalar $\alpha$. The penalty is designed so that the cost function maintains its convexity; in fact, in the case of the quadratic cost functions for the DERs, the function remains strongly convex.

\subsubsection{Implementation Methodology}

The proposed methodology implements an online no-load frequency adjustment method in the lower level as the primary control. A continuous distributed optimization through the diffusion strategy is implemented in the upper level as combined secondary and tertiary controls.

Droop control represents a simple yet powerful solution to the microgrid primary control problem. DERs adjust their power output according to the no-load speed setting with respect to the system frequency. By changing the no load frequency upwards or downwards, the power output for any given system frequency can be controlled. The noload frequency of a given generator can be set to obtain any desired power output according to its droop slope $R$. 


$$
\begin{gathered}
f_{N L}=P\left(f_{N L}-f_{F L}\right) /\left(P_{\max }-P_{\min }\right)+f_{s y s} \\
f_{N L}=-P . R+f_{s y s}
\end{gathered}
$$

Here, $R$ is typically formulated with the maximum and minimum power outputs of the DG, $P_{\max }$ and $P_{\min }$, respectively. The no-load and full-load frequencies, $f_{N L}$ and $f_{F L}$ respectively, are normally chosen as the bounds which the system frequency must not cross. The secondary and tertiary optimization level controls adjust the no-load frequencies as shown in Figure 9.8. For the energy storage systems, the no-load frequencies can be set below nominal to absorb power (charge) or above nominal to inject power (discharge).

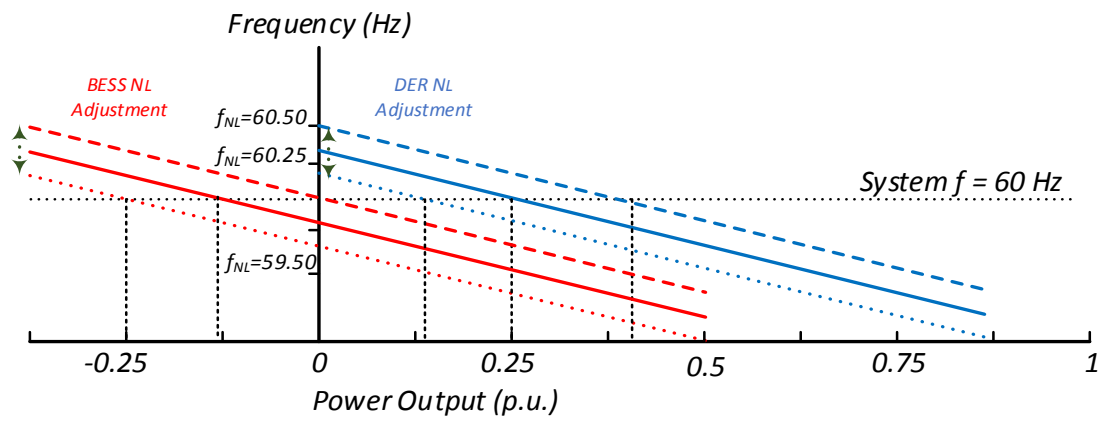

Figure 9.8 Droop no-load frequency adjustments.

In the upper level control, the main functions of the stochastic and dispatchable agents are illustrated in Figure 9.9. Assuming agent $i$ is stochastic, the device layer reads the current power $\left(P_{i}\right)$ being demanded or produced by its associated device.

The information sharing (IS) diffusion takes this information $\left(x_{i}\right)$ and transmits it to its neighbors and receives the same from them $\left(x_{j}\right)$. Convergence is achieved once a certain precision level has been achieved, at which point the net power $\left(P_{N}\right)$ required by the microgrid is known. 


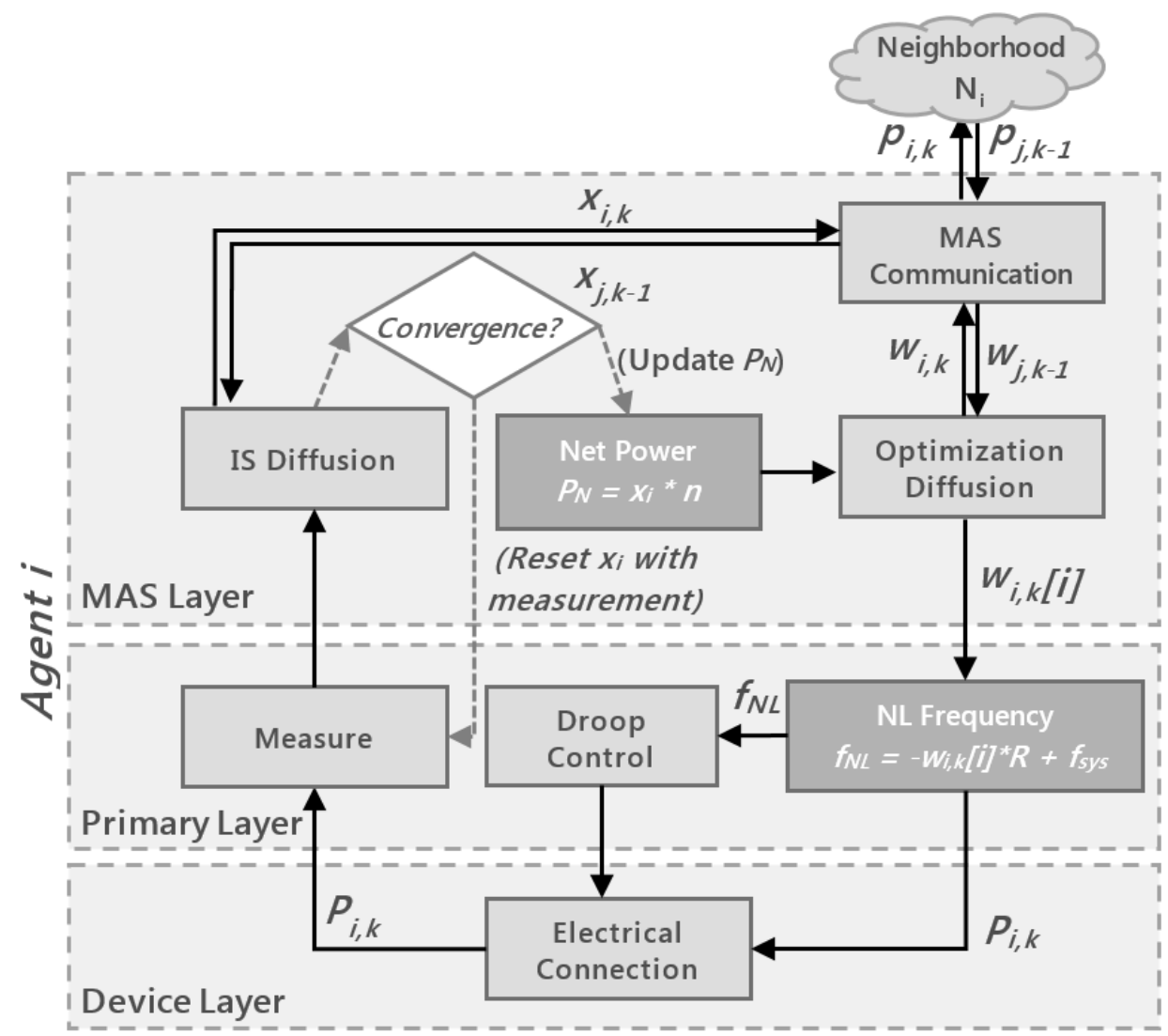

Figure 9.9 Agent operation flow

Each net power update is used by the dispatchable agents according to equation 9.21. However, optimization diffusion is continuously running and all agents participate in disseminating the $w_{i}$ estimates across the microgrid. The data packets $\left(p_{i}\right)$ are a combination of the IS and optimization diffusion data points which are sent and received among the neighborhood of agents. After each iteration, the no-load frequency of the droop control for dispatchable agents is updated to track the optimum. Therefore, the upper level control is setting the output power points of the DERs at their optimum levels by manipulating the droop curve. In turn, this adaptive droop scheme inherently takes care of primary frequency control in an instantaneous and autonomous way. 
Since reactive power cannot be billed in the traditional way active power is, voltage regulation was not considered within the optimization problem. Both in simulation and experimental studies, we have implemented conventional synchronous generators and energy storages as (V-f) control.

Synchronous generators are equipped with automatic voltage regulators. Energy storage inverter-based DERs implement reactive power compensation for the local bus. DERs regulate the bus voltage control while maintaining the frequency regulation with the earlier described distributed control method.

\subsection{Simulation and Experimental Results}

\subsubsection{Simulation Results}

The 9-node microgrid depicted in Figure 9.1 was built in the Matlab Simulink simulation platform. The stochastic load demand and renewable generation output were programmed into the model according to real-world profiles. The two conventional DERs and an energy storage system (ESS) are controlled through the described adaptive droop strategy and the diffusion communication algorithms were implemented in the model.

Table 9.2 DER coefficients for simulation

\begin{tabular}{|c|c|c|c|}
\hline \multirow{2}{*}{ DER } & \multicolumn{3}{|c|}{ Parameters } \\
\hline DG1 & $\mathrm{a} 1=30$ & $\mathrm{~b} 1=4 \mathrm{e}-8$ & $\mathrm{c} 1=6 \mathrm{e}-11$ \\
\hline DG2 & $\mathrm{a} 2=20$ & $\mathrm{~b} 2=5 \mathrm{e}-8$ & $\mathrm{c} 2=10 \mathrm{e}-11$ \\
\hline ESS & $\mathrm{x} 2=20$ & $\mathrm{y} 1=8 \mathrm{e}-5$ & $\mathrm{z} 1=8 \mathrm{e}-11$ \\
\hline
\end{tabular}


The coefficients for these DERs corresponding to equation 9.1 and equation 9.4 are specified in Table 9.2, they were analytically selected to approximate real-world conditions. Delays were also considered to simulate the communication lags. IS performance of the diffusion strategy was evaluated and compared to the consensus-based control. As illustrated in Figure 9.10, the microgrid net power $P_{N}$ is being diffused through the 9 node network. The net power profile is similar to the residential load profile, due to large solar and wind power outputs around noon, which compensate for a big portion of commercial and critical loads.
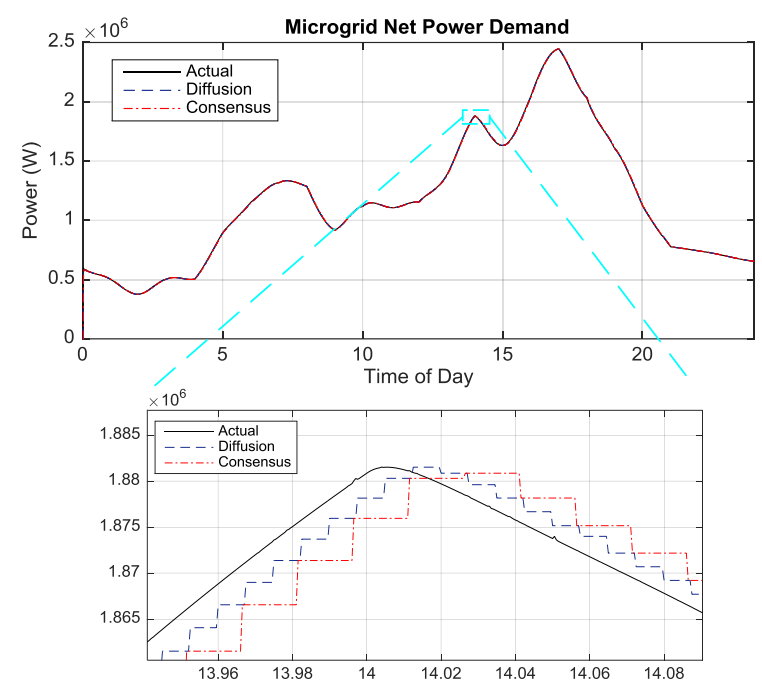

Figure 9.10 Information sharing performance comparison.

Both diffusion and consensus closely track the actual net power demand. However, in the zoomed figure, it is clearly seen that the diffusion strategy is approximately twice as fast to converge as consensus, which agrees with the earlier performance comparison.

Although the units in the x-axis correspond to the hours of the day, the whole simulation was ran in 48 seconds, with iteration steps of $5 \mathrm{e}-4 \mathrm{~s}$, which would correspond to about one iteration per second in real-time. As previously mentioned, modern peer-to-peer 
communication technologies can achieve very high speeds of transmission. Given the low data payload necessary for the messages in the proposed framework, the tracking of the net power and economic dispatch points would be much more accurate than presented in simulation results. The optimization of the cost functions are compared versus a centralized solution in Figure 9.11. As can be seen, the cost directly corresponds with the net power demand. It is also shown how the total cost of the distributed control scheme closely tracks that of the centralized scheme (which represents the absolute optimum given system-wide conditions and cost knowledge).

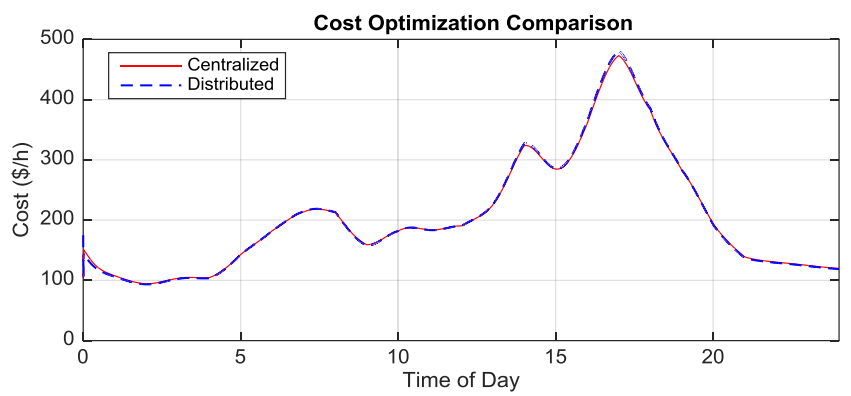

Figure 9.11 Cost optimization comparison (centralized vs diffusion).

Table 9.3 presents the aggregated costs over a 24-hour period for an islanded microgrid. The proposed distributed optimization scheme is validated as the total cost is only $0.11 \%$ above the centralized solution.

Table 9.3 Total cost comparison

\begin{tabular}{|c|c|}
\hline Control Scheme & Total Cost \\
\hline Centralized & $\$ 4,917.60$ \\
Distributed MAS & $\$ 4,922.80$ \\
\hline Difference & $0.11 \%$ \\
\hline
\end{tabular}


To provide some real-world context, the average cost per MWh for this scenario is $\$ 171 / \mathrm{MWh}$ or $\$ 0.171 / \mathrm{kWh}$ (with an average load of around $1.2 \mathrm{MW}$ and total energy requirement of $28.7 \mathrm{MWh}$ ). This is high compared to current electrical rates in the US markets, however this would be an islanded microgrid that would not benefit from bulkgeneration economies of scale.

To scrutinize the performance of the proposed control, the most drastic event, an unintentional islanding, was demonstrated. In grid-tied operation, the net power demand was low and the DERs were operating at their optimum points considering the constant marginal cost of the utility electric rate.

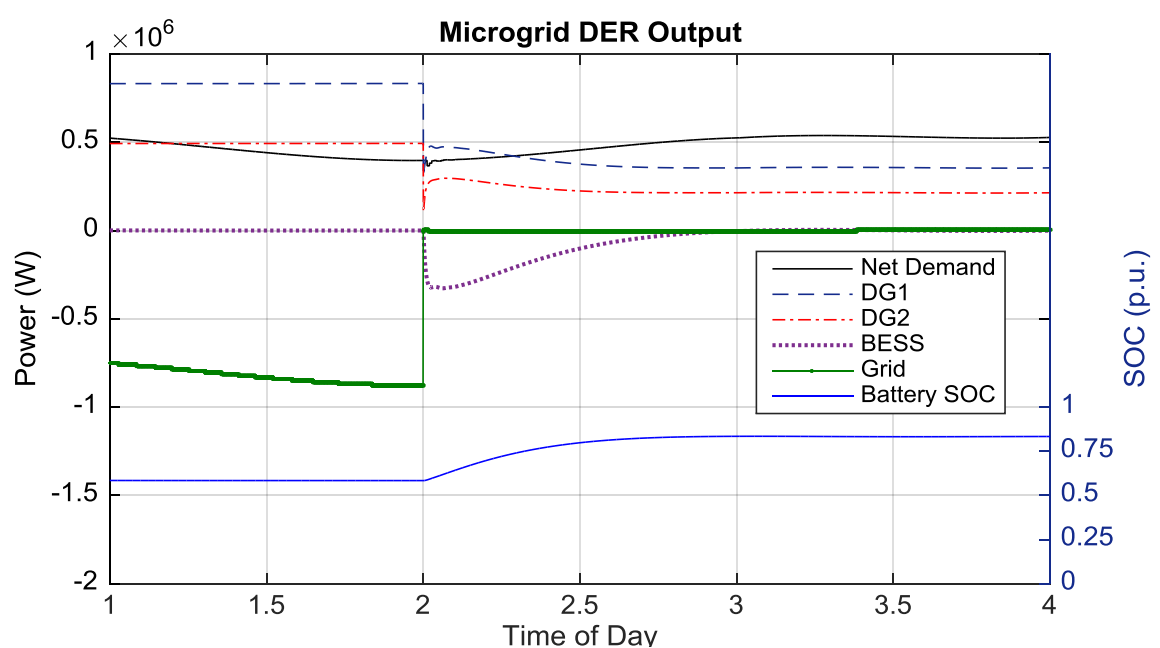

Figure 9.12 Microgrid islanding power output and battery state of charge.

Initially, the microgrid was exporting power to the external grid and the energy storage had already reached an equilibrium on its state of charge due to the fixed marginal cost. As shown in Figure 9.12, exactly at 2 AM, the PCC of the utility grid is opened and the power export was terminated. As the lower level control, the droop controllers act immediately by reducing the outputs of both DERs and the storage system started to absorb 
some amount of the power into the battery system. Then, as the upper level control, the diffusion strategy takes over by adjusting the no-load reference points of the DERs.

First the secondary response recovers the nominal frequency after the grid agent drops out of the $w$ vector and the remaining DERs need to maintain the net power demand balance. Then, the optimization process continues adjusting the assets to their economic dispatch points according to the changing net power demand within the microgrid. Since the net power demand was relatively low at this time, the marginal cost of the microgrid (optimal $\lambda$ point) was also low (lower than the utility rates). The battery not only absorbs power due to the droop controller, but continues to charge until finding a new equilibrium SOC that moves with the net demand.
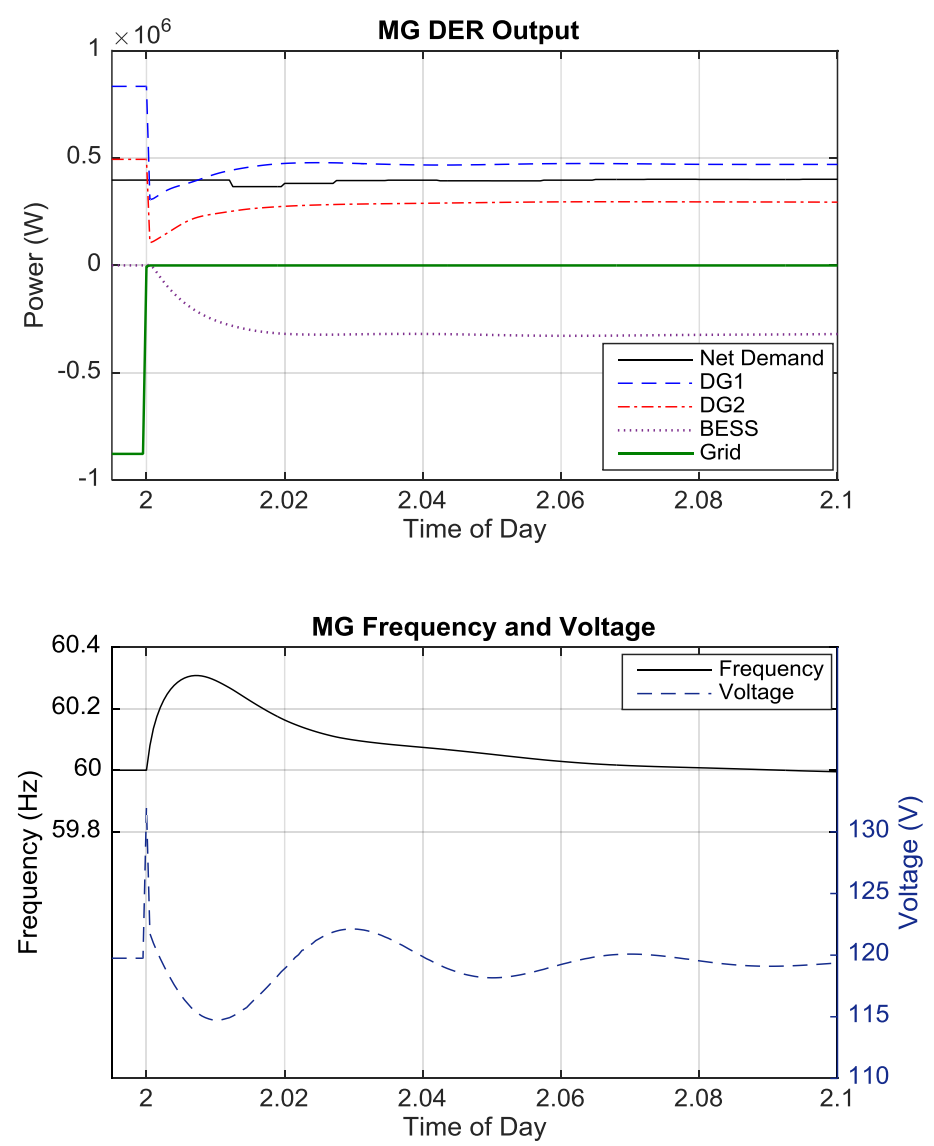

Figure 9.13 Microgrid islanding transient analysis. 
Due to the extra power being generated during the islanding event, the frequency of the system rises to $60.3 \mathrm{~Hz}$ as shown in Figure 9.13. The primary droop control suppresses the initial frequency rise by immediately dropping the power outputs of all dispatchable assets to protect the system from going beyond acceptable conditions as seen in the zoomed-in Figure 9.13.

Once the frequency is suppressed, the secondary control kicks in through the MAS to mitigate the residual error and put the system back to the nominal frequency value. The voltage shoots up further around $10 \%$ up to $132 \mathrm{~V}$ as a transient, but is kept within safe levels. Diffusion then keeps the frequency very close to the nominal as the net demand fluctuates.

To compare the diffusion strategy with a centralized solution, the adaptive droop no-load frequency adjustments were compared side by side. The centralized solution implements the adaptive droop adjustments to operate the asset at the exact economic dispatch point given global power system knowledge. In Figure 9.14, the slight differences are observed. Although the diffusion adjustments fall a little behind, the accuracy was still $99.9 \%$ at the worst points.

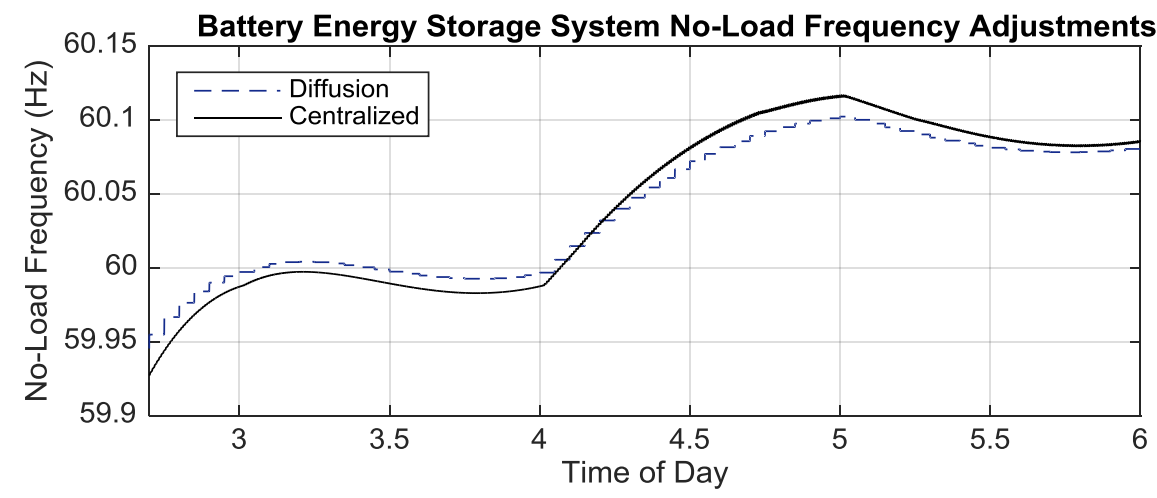

Figure 9.14 Battery energy storage system no-load frequency adjustments. 


\subsubsection{Experimental Results}

To demonstrate the capability of the proposed control strategy in real-time, an experimental study has been performed on the developed microgrid. The industrial agent framework was used as the cyber-physical platform to implement hardware-in-the-loop agents. Information sharing is implemented by reading the power being drawn or injected by loads and generators through intelligent electronic devices (IED). An OPC UA middleware framework was implemented to map measurements in JADE Java agent platform. Each agent communicates to the others through the JADE and OPC UA middleware to reach convergence on the net power requirement of the microgrid. Here, the implemented agent framework simulates the peer-to-peer communication infrastructure that we envision in a real-world scenario. Consequently the power points of the DERs are adjusted to their economic dispatch points through the diffusion optimization. Both information sharing and optimization diffusion is sent in the same data packet. Figure 9.15 illustrates the experimental performance of the proposed diffusion strategy under the islanding event. In this scenario, we defined a 9 node microgrid network as initially importing power from the utility grid. The islanding event is established by opening the PCC circuit breaker. The primary control was taken immediately by all dispatchable DERs to recover system frequency and voltage. 


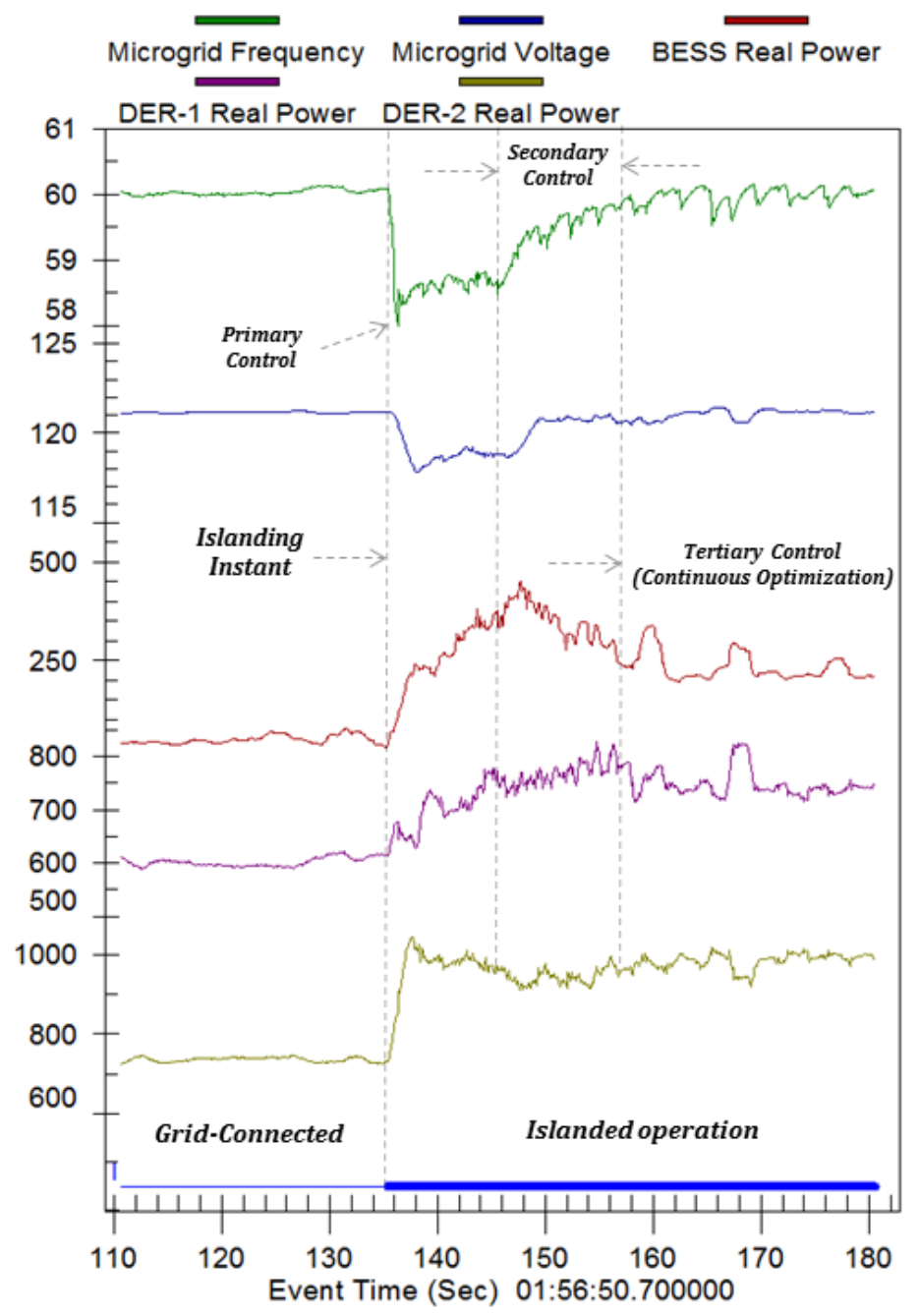

Figure 9.15 Experimental islanding - distributed optimization performance.

Once the system frequency was settled, the secondary control moved in to perfectly mitigate the residual frequency error and put system frequency back to nominal value.

In Fig. 18, the islanding event can be seen happening at around 135s. Primary droop control immediately takes action by injecting more power from the DERs and Battery Energy Storage System (BESS); this happens in less than a second and the frequency is prevented from collapsing. Then, secondary control recovers the frequency to the nominal $60 \mathrm{~Hz}$ by adjusting the no-load frequency parameters of the droop curves for each asset. Secondary 
frequency recovery is accomplished around 155s. Subsequently, the tertiary control economic dispatch is continuously adjusting the assets to their optimal levels.

It should be noted that the system frequency is more stable in grid connected operation, whereas minor frequency oscillations were observed in islanded operation mode due to the particularly low inertia of the experimental microgrid. Afterward, the tertiary control continuously adjusts the operating points until the optimum is reached.

\subsection{Conclusion and Summary of the Result}

The distributed diffusion strategy and its great potential value to the microgrid optimization problem have been discussed. The proposed method was applied to a 9 node microgrid net-work including stochastic and dispatchable DERs. It has been shown that the fully distributed multiagent systems based on the diffusion strategy can be easily applicable for microgrid optimization. The results show that in comparison to developed agent-based diffusion strategies and consensus-based methods, the new microgrid optimization scheme presents a desirable performance. It has been concluded that the proposed strategy can be successfully implemented in actual power systems offering a superior decision making and improved performance on optimal microgrid control. 


\section{MULTIAGENT-BASED GAME THEORETIC REVERSE AUCTION MODEL FOR MICROGRID MARKET OPERATION}

\subsection{Introduction}

In this chapter, the grid-connected real-time operation of a multi-agent based microgrid system is studied for market operations of renewable and conventional resources. A multi-agent based platform incorporating PMU measurements is utilized to monitor and control microgrid operations through sensors and actuators of generation units, such as the governor and inverter control of the power storage device. A multi-agent based game theoretic reverse auction model is utilized to enable a competition between DERs in order to obtain the cheapest power supply for a lumped load on a microgrid. The realistic study is successfully implemented and operated at the previously developed microgrid.

The conventional electricity distribution networks encounter a high penetration of commercial-based renewable energy and distributed resources. Smart grid and active distribution networks are the terms commonly used to express the operation of renewable energy resources' penetration to the power network. The term smart grid is introduced as an intelligent energy supply system and comprises the networking and control of intelligent generators, storage facilities, loads with the aid of information and communication technologies [140]. An era of major transition of existing electricity networks from passive to active distribution networks with distributed energy resources (DER) results in bidirectional electricity flow [141]. This transition brings many challenges on operation due to the bidirectional power flow and intermittent source of renewable energy generation. 
However, DERs can significantly impact the reliability of power systems as long as they are operated efficiently. Active distribution networks offer a solution for demand-side management, since they have the ability to partially control local generation capacity incorporated with storage devices and dispatchable generation units. The DERs can also be utilized to reduce peak demand costs by enabling them to operate only during peak hours to reduce transmission network congestion and stress. Directly grid-connected renewable DERs can be considered negative load sources with an intermittent generation profile. This uncertain generation profile should be controlled by the assessment of optimal unit scheduling. Economical unit commitment, scheduling of the DERs and power sharing between multiple units are the most important concerns of active distribution network operations in open-market conditions.

Microgrids are small-scale electricity networks comprised of diverse distributed generation units, which can operate in grid-connected or islanded mode. Microgrid economics are highly dependent on local conditions, such as availability of fuel, gas, wind, and solar irradiation. In an open-market condition, utilities are no longer monopolized hence DER stakeholders are the private entities that can compete with regional utilities. The electricity trade can be handled locally in a region by several DER investors or crossborder trading by utility wholesale markets and neighboring regional systems [142]. In distribution networks, feeder-based electricity trade is the ideal trade choice to avoid transmission route and loss costs.

A vast number of DERs should be able to operate incorporating a large number of complicated operational functions. The new generation grids require fast and intelligent decision-making algorithms with an efficient communication infrastructure, since power 
system operators are inefficient in dealing with highly active and constantly varying operations in future grids. Multi-agent based decision making algorithms incorporating real-time communicative devices offer a reliable solution for unit commitment in competitive market conditions. Agent based marketing models have applications on a variety of scales, from large bulk generation units to small home-based entities [143]. There has been a growing interest for agent-based market scenarios that maximize the benefits of generation units by analyzing the effects and evaluation of bidding strategies [144-146]. Learning and predictive agent implementations have been studied in simulation environments to enable profit-maximizing agents [147].

Thus far, the applications of multi-agent systems have been deployed in simulation [148] and prototype laboratory applications. Few efforts have been put towards the implementation of multi-agent systems in real-time utility or industrial applications in power system engineering [149], [150]. Aligned with the maturing technology, new methods should be discovered to enable utility and industrial multi-agent system applications. Hence, multi-agent systems should be able to operate with existing industrial components and future power system assets. PMU, DERs and IED are the main assets of future electric power systems. Aging network structure should be rehabilitated by replacing or adding new components into the system. Currently, the costs of PMUs and IEDs are high for particular applications, such as distribution network deployment; however, it is expected that the costs will decrease with maturing technology and new market players. Such new components would maintain sustainable, reliable and efficient operation of future generation grids. 


\subsection{Game Theoretic Reverse Auction Model}

The main goal of restructuring the electricity market is to provide benefits to society by enabling a competitive environment. Volatility of fuel/gas prices and weather conditions shapes the DER stakeholder's attitude towards operation time and duration of the power generation. The market structure has three entities - sellers, buyers and aggregators. An effective way to decide which seller qualifies to supply the required demand for the system load is to implement trade contracts. There are two main types of contracts available: the day-ahead market and hour-ahead mechanism. In the day-ahead market, the auction is organized to balance the required amount of power for the following day; similarly, in an hour-ahead mechanism, the auction interval takes place on an hourly basis. The availability of generation units, bids and trades are determined each hour. A DER can be deployed with a fixed price in a monopoly market. However, for an ideal competition environment, a wider policy should be established.

An auction is a public sale in which property or merchandise is sold to the highest bidder by determining resource allocation according to bids received from participants. The auction can be either for buying or selling the electrical commodity. A procurement auction, or reverse auction, is the process that has been ruled by an entity, which announces its interest to purchase a certain amount of product, and solicits competitive bids to acquire it with the lowest cost. Several countries have used reverse auction models to enable a competitive renewable energy deployment in their systems [151]. Auctions can be open or seal-bid wise. In an open auction model, all participants know what the other participants are bidding on. In a one-sided auction model, only buyers or sellers can submit the bids, where a two-sided auction model allows both buyer and seller to submit bids. The bidding 
process can be once or continuously until it reaches an equilibrium point. Designing a proper auction model is a difficult process. There are many questions that need be addressed prior to designing an appropriate auction model. Some of them can be stated as follows: the number of the objects to be auctioned, the reserve price, the acknowledgement of the reserve price, the way bids are collected, and the qualified bidder selection.

Most common auction models are English, Dutch and Vickrey [152]. A reverse auction is a model in which prices go down in contrast to typical auction models. Normally, in a reverse auction, multiple sellers bid to sell goods to a single buyer. However, more than one seller could be picked as qualified bidders when a single seller cannot meet the required demand. Although there are a variety of implemented auction models, it has been realized that reverse auction is a useful tool in power system market operations.

The contracts can be done bilaterally or using the pool approach. Many game theory auction models are available. Two main types are individual and cooperative games. In contrast to an individual game, in a cooperative game, players (agents) would behave in a cooperative manner; hence the game is a competition between coalitions of players to fix a problem, which influences all players in the game. As a power systems example, the frequency of an island should remain stable during the islanded operation; hence supply and demand should match. Cooperative DERs should supply the required demand in order to maintain the stability of the system regardless of the cost of electricity. However, in a grid-connected mode of operation, if the price of DERs is higher than the utility price, then none of the DERs join the game since the frequency will be regulated by the stronger utility grid. This example can be considered as an individual game. In this study, multiple gridconnected DERs compete to sell electricity for a lumped load in a microgrid. 


\subsection{Microgrid Agent Modeling for Market Operation}

The IEEE guide 1547.3 addresses the guidelines of monitoring, information exchange and control of DERs interconnected to electric power systems. In this chapter, we followed these guidelines to enable the proposed methodology to be compatible with existing rules. The DER stakeholders are in interaction with the area electric power system operator (AEPSO), DER aggregator, DER maintainer, and DER operator.

AESPO is the entity responsible for safe and reliable operation of the power system. It is necessary to interact with DER units in the system by means of exchanging messages regarding the system status. Utility grid interface and distribution line models are properties of AEPSO.

DER Aggregator is a centralized controller that manages multiple DERs for main and ancillary services. The lumped load model is managed by a DER aggregator by organizing the hour-ahead reverse auction model.

DER Maintainer is responsible for the safe operation of the DERs by enabling a maintenance schedule. The availability of a generation unit can be defined by the DER maintainer.

DER Operator is the main entity responsible for local control of generation units. Monitoring and measurement power system parameters and controlling the outputs, such as a governor, is handled by the DER operator. The DER operator manages the sensors and actuators.

Interoperability of the stakeholders with a multi-agent approach is highly dependent on the information exchange capability of the field devices, such as sensors and actuators. An interface is the point of contact that a software component has with its interacting 
partners. Some interfaces are defined by protocols where they have a generic set of services. Protocols are a set of rules for communication of industrial devices. Electricity network field devices are manufactured by different vendors. Each vendor equipment has its own communication protocols to interact with the outside world. This problem is one of the main challenges for the implementation of multi-agent based decision making platforms in the existing grid infrastructure. In this study, a set of industrial communication protocols and physical layers are used to interact with the agents.

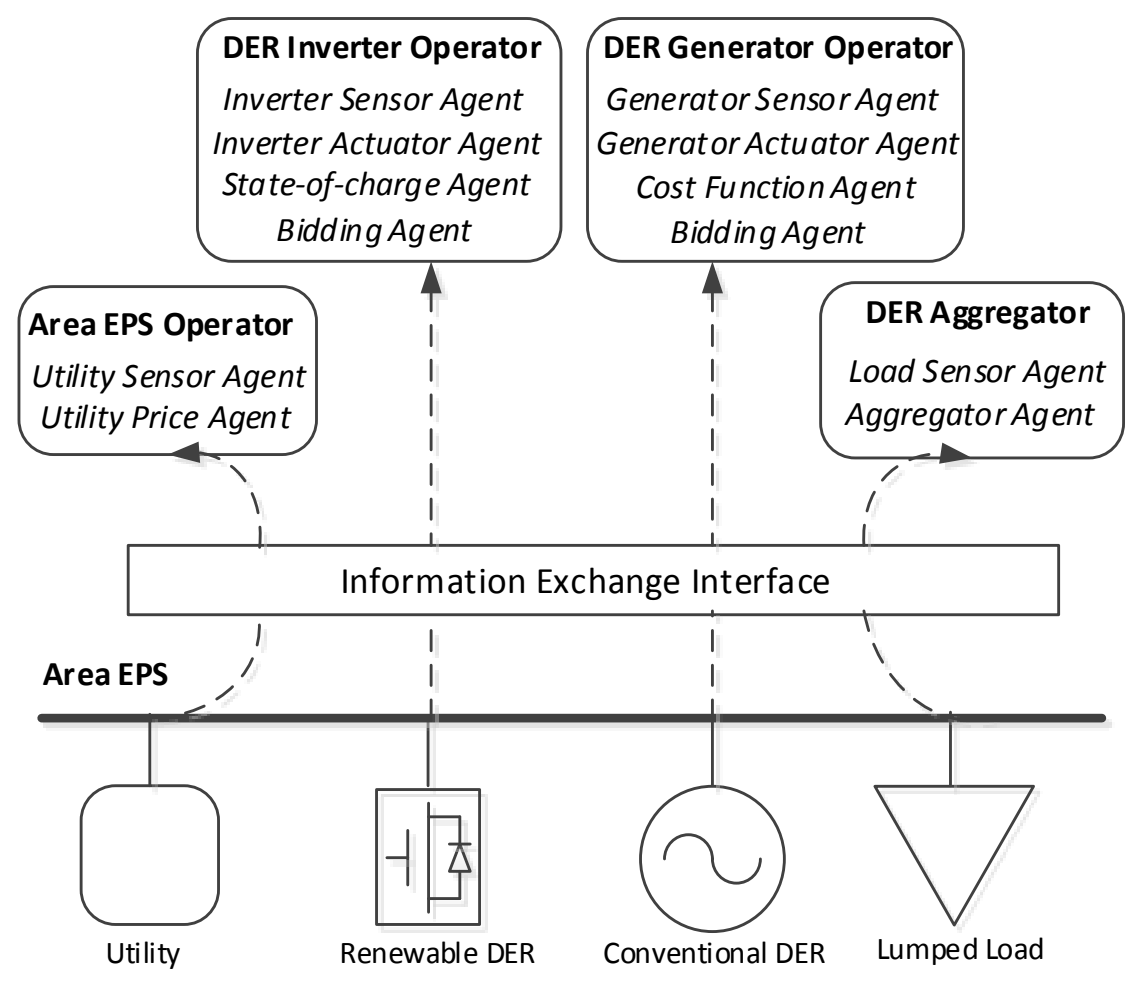

Figure 10.1: Defined Agents for auction scheme.

Figure 10.1 shows the implemented agents on the Jade platform to operate the complete system. Sensor agents are responsible for system parameter measurements and sending the information to other agents or upper control areas if necessary. Actuator agents are responsible for operating generation units according to the power reference received 
from the bidder agents. Bidder agents inform the actuator agent duration and power generation amounts if they are qualified bidders after the auction process is organized by the aggregator agent. All available bidder agents register to the yellow page so that the aggregator agent can communicate with them. Yellow page is a service in which an agent can find other agents providing the services he requires in order to achieve his goals. If the DER is available for the next hour, the bidding agent decides whether to join the auction or not according to base price information received from the cost function or the state-ofcharge agents.

The cost function agent is specifically defined for a fuel/gas-dependent generation unit, which is a synchronous generator in this study. The fuel/gas price is assumed to be volatile; hence generation cost is varying. Each conventional generator has a quadratic cost function which affects the cost of electricity according to volatile fuel costs for different hours. " $i$ " is the unit number, " $P$ " is the electrical output and " $a, b, c$ " are random varying fuel coefficients.

$$
F_{c}(P)=\sum_{i=1}^{n} a_{i}+b_{i} P_{i}+c_{i} P_{i}^{2}
$$

Renewable resources are assumed to be coupled with storage devices. According to the state-of-charge of the batteries, the mood of the agent changes from being greedy, moderate and relax. The base price changes according the mood of the agent, which determines the duration of the stay of an agent in the auction. Table 10.1 shows the base prices of the inverter bidder agents and their dependence on mood. The utility cost agent basically broadcasts the utility electricity cost for each hour. During the peak hours, it is assumed that the cost of the electricity is much higher than the regular hours of the day. In 
this study, utility peak and regular hour prices are defined as 0.33 and $0.14 \$ / \mathrm{kWh}$, respectively.

Table 10.1: Inverter bidding agent modes.

\begin{tabular}{|c|c|c|}
\hline State-of-Charge & Agents' Mood & Lowest Bidding \\
\hline $0-30 \%$ & Relax & $0.15-0.13 \$ / \mathrm{kwh}$ \\
\hline $30-60 \%$ & Moderate & $0.13-0.11 \$ / \mathrm{kwh}$ \\
\hline $60-100 \%$ & Greedy & $0.11-0.08 \$ / \mathrm{kwh}$ \\
\hline
\end{tabular}

The aggregator manages the communication and the information exchange between participants to minimize the cost of the electricity to be purchased for the lumped load. The aggregator agent is the main entity operating the auction process. Its task is to solicit bids from the agents registered to the yellow pages, and to invite them to participate in the auction. The ultimate goal is to purchase the required amount of power with the lowest cost. DERs submit bids to the DER aggregator, declaring the power available to sell and the price for the next hour. Each DER has a generation capacity, marginal cost, and availability. A reverse auction model has been implemented and realistically operated. Table -10.2 briefly expresses the simplified pseudo code of the proposed reverse auction model. the process is continuous unless the program is terminated. All available generators in the microgrid should register to the yellow pages in order to participate in the bidding process. The required amount of load is assumed to be correctly estimated for each upcoming hour; therefore the aggregator agent has this information ready to inform all agents registered to the yellow page. The main purpose of the aggregator agent is to purchase the required amount of energy with the least cost. 
Table 10.2: Reverse auction agent negotiations.

\section{Begin}

Initialize: All available generators register to yellow page;

\section{Loop}

Aggregator agent: inform utility base price, power demand for next hour;

Aggregator agent: request bids;

if (DER base price < utility base price) then

Bidder agents: inform submit bids;

else

Bidder agent: drop not participating;

end

until (demand $=>$ total bid submitted supply) do

Aggregator agent: inform new base price;

Aggregator agent: request new bids;

Bidder agents: inform submit bids;

end until

Aggregator agent: inform market clearing price;

if first qualified bidder covers all demand then

Aggregator agent: agree send power reference to qualified bidder;

else

Aggregator agent: agree send power references to first and the other qualified bidders; 
The aggregator agent collects the submitted bids and checks whether the submitted supply matches the demand. If the supply is higher than the demand, the aggregator sends a new bidding request to the bidding agents by lowering the price. If bidding agents are still interested in the new submission, they submit the new price; otherwise they drop and quit from the auction for that particular hour. The process continues until only one qualified bidder remains in the auction. Accordingly, the market clearing price is established, which is the submitted price of the last second qualified bidder, as in the Vickrey auction model. One more process is defined to check whether the qualified bidder's submission is able to cover all demand. The first qualified bidder is supposed to cover the majority of the total demand. However, if there is still a deficit of supply, the second qualifying bidder covers the remaining amount. Once the auction process is over, the aggregator informs the final power references to the qualified bidders. Accordingly, bidder agents inform actuator agents to increase generation for next hour. The same process is repeated for each hour.

If all base prices of the generation units in the microgrid are higher than the utility base price and none of them are interested in participating in the auction, the aggregator agent buys all required power from the utility. If the total supply is less than the demand and some of the bidders are participating in the auction, the aggregator agent buys a qualified amount of power from the DERs and the remaining is purchased from the utility. While managing the power sharing of many generation units, the power system quality should be maintained. Frequency and voltage stability of the system are the main concerns for continuous operation.

A microgrid can be operated in both grid-connected and islanded mode. While utility connection is available, voltage and frequency are regulated by the grid. 
Synchronous generator and inverter based renewable generation units are operated by a power generation reference.

In this section, only grid connected operation mode is studied. Active power balance is directly related to system frequency; hence it should be regulated by the utility if proper load sharing may not be established by DERs. The dynamic model for frequency is given as below, where $\mathrm{H}$ is the system inertia constant in seconds, $\omega$ is the electrical angular velocity or frequency, and $P m$ and $P e$ are mechanical and electrical powers, respectively.

$$
2 H_{t o t} \frac{d \omega}{d t}=P_{m}-P_{e}
$$

The synchronous generators have inertia, however inverter-based DERs do not. Even though the sum of assigned power references to all generation unit matches the demand and the supply in theory, losses and other system behaviors would result in an improper power balance in a real-time implementation. This mismatch would result in a frequency drift inside the system. Therefore, in utility connected mode, the remaining power is drawn from the utility grid. Power line losses are an important factor, where large scale power generation units and long transmission lines exist. However, the power line losses can be neglected in short feeders, since DERs are spread all over the feeder. In this study, line losses are drawn from the utility.

\subsection{Hardware Setup for Market Agents' Implementation}

The agent platform, generation units, loads, transmission line models, field sensors and actuators are implemented in a reconfigurable small scale power system available at Florida International University, Smart Grid Test Bed. 


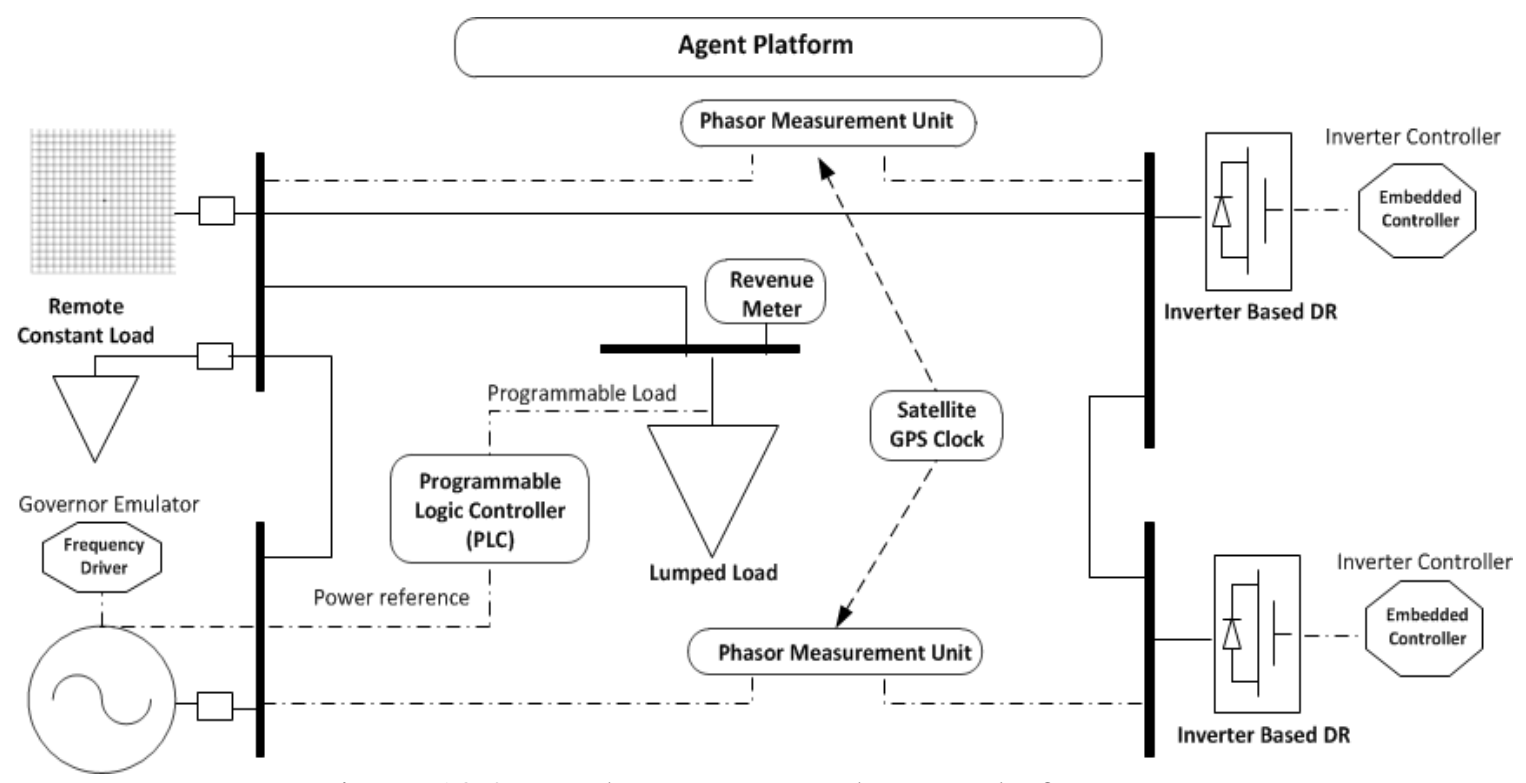

Figure 10.2: Hardware setup and agent platform.

The system under study is shown in Figure 10.2. PMUs have become a reliable measurement and monitoring choice for electric power systems since they provide timesynchronized measurements. Local frequency, voltage, current and power measurements are the main available measurements provided by PMUs. This has been enabled by the availability of a global positioning system (GPS). Thanks to precise sampled data, the measurements from different system buses are comparable. Two PMUs and an energy meter are deployed on the system as sensor devices. PMUs are capable of measuring two network buses at the same time since they provide two analog measurement channels. Four generation buses are monitored by PMUs and the lumped load model is monitored by the energy meter. In order to understand and visualize the overall view of the complete distribution network, exact time synchronization for all measurement is necessary. A satellite clock is available for PMUs to get time-synchronized measurements. Apart from readings in the agent platform, a phasor data concentrator (PDC) is implemented to measure system parameters through the PMUs. The PMU time-synchronized data update 
rate is adjusted to 5 messages per second. Voltage variation at the utility common coupling point is also monitored in real-time. A PLC is used to enable a load-switching function according to a predetermined load profile. An AC synchronous generator model is used for experiments, which is a $13.8-\mathrm{kVA}, 60-\mathrm{Hz}, 208-\mathrm{V}$ and $1800 \mathrm{rpm}$ synchronous machine. The Automatic Voltage Regulator (AVR) model is a half-wave phase-controlled thyristor-type regulator. Excitation current is derived directly from the generator terminals. The synchronous generator turbine shaft is directly coupled to an induction motor to emulate a governor model. A frequency driver provides the required amount of torque to the induction motor, hence the synchronous generator operates. An inverter-based DER is interfaced to the ac microgrid via a bidirectional ac-dc/dc-ac converter to allow bidirectional power flow between ac and dc sources.

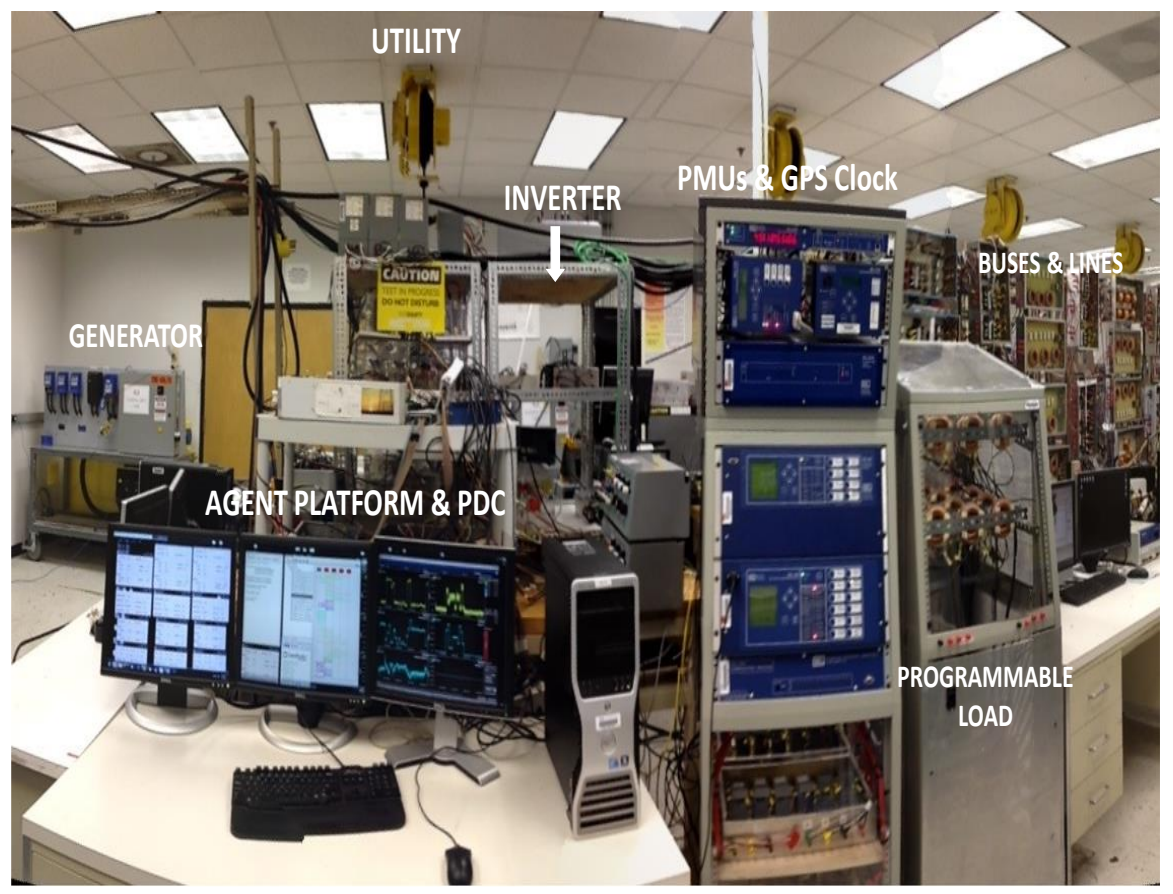

Figure 10.3: Auctioning agent hardware setup. 
A 5-kW programmable dc power supply is used to emulate a typical renewable energy resource. This power supply can be considered as a solar or wind resource. The complete inverter system control is enabled by the digital signal processor (DSP)-based platform dSpace Control Desk. A variety of industrial communication protocols have been implemented to acquire field data and to provide information to the actuators. The agent platform is located at a central PC with a peripheral component interconnect (PCI) express card mounted to enable the use of multiple serial communication ports. Each agent is assigned to communicate through individual serial or Ethernet ports. The utility, generator, inverter1 and inverter2 sensor agents' access bus parameter readings by using IEEE Std. C37.118 synchrophasor data transfer protocol. The load sensor agent is interfaced to the energy meter, which uses Schweitzer Engineering Laboratory (SEL) Fast Message protocol. Modbus is a de facto standard protocol, which is used by many power systems and industrial electronic device vendors. Agent platform and PLC communication is established by Modbus TCP protocol through Ethernet ports. To control inverter1 and inverter2 actuator agents, a raw data exchange between the PC and embedded controller is established with a RS232 serial port. Modbus RTU is used to communicate with the generator actuator agent through variable frequency drive, which emulates synchronous generator governor model.

\subsection{Reverse Auction Game Results}

The game is played for approximately 1 hour in a real-time experiment, where each hour of the twenty-four hours of the day is assumed to last 2 minutes. Table 10.2 shows the random output of the played game in the microgrid. The predefined daily load profile 
shows a regular day's power demand in a household, which has two specific peak hours, in the morning and evening. As seen from Table 10.3, the system experiences a morning peak between 07.00 and 09.00 , and an evening peak between 18.00 and 19.00. Utility peak and regular hour prices are defined, respectively, as 0.33 and $0.14 \$ / \mathrm{kWh}$. The generator is assumed to be always available. The availability of inverter-based DERs depends on the solar irradiation and stored energy. Generator 1 has a volatile operation cost, which is defined by a random cost function. The irradiation profile is randomly generated in a software, which emulates the different weather and shadow conditions. The current charge value shows how much power is stored in the battery system. If a unit is unsuccessful in bidding in the auction and cannot discharge generated power, its irradiated power is stored cumulatively. The battery storages of the Inverter1 and inverter2 are limited to $600 \mathrm{kWh}$ and $900 \mathrm{kWh}$, respectively. According to the state-of-charge of the batteries, the mood of the agents change, hence the base price changes linearly. The utility price is assumed to be higher during the peak hours compared to the regular hours. This enables renewable resources and generators to compete in supplying power for the lumped load. It can be seen that the auction is more competitive during the peak hours, such as the $16^{\text {th }}$ hour. When renewable DERs are online at peak hours, the market clearing price is less than the utility base price, which means the aggregator agent would purchase electricity at a cheaper cost.

Figure 10.4 illustrates the aggregator agent auction progress during the $16^{\text {th }}$ hour in order to determine the qualified bidders for the following hour. The demand for the $17^{\text {th }}$ hour is anticipated as $600 \mathrm{~kW}$ and the utility base price is $0.33 \$ / \mathrm{kWh}$. The auction starts 1 cent lower than the utility base price. The aggregator agent solicits bids from the registered DERs in the yellow page. 
Table 10.3: One hour random game output.

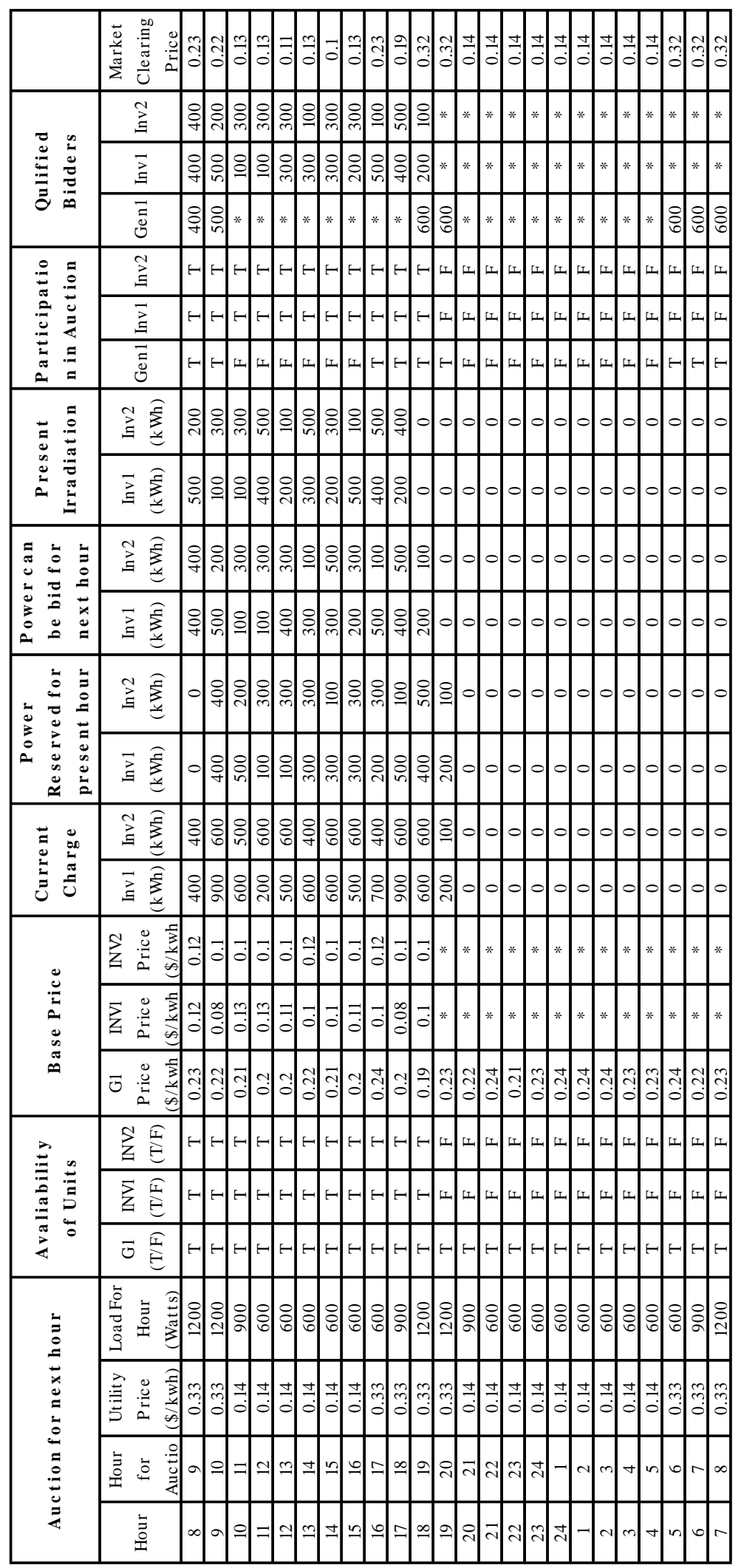




\begin{tabular}{|c|c|c|c|}
\hline \multirow{2}{*}{$\begin{array}{l}\text { State: Done } \\
\text { Required Power: }\end{array}$} & & \multirow[b]{2}{*}{ Clearing Price: } & \multirow[b]{2}{*}{0.23000008} \\
\hline & 600.0 & & \\
\hline \multicolumn{4}{|c|}{ Starting auction at base price: $\$ 0.32000002$} \\
\hline \multicolumn{4}{|c|}{ Sending initial status request to registerd bidders: } \\
\hline \multirow{2}{*}{\multicolumn{4}{|c|}{$\begin{array}{l}\text { GeneratorBidder owned by DRGenerator } \\
\text { Inverter1Bidder owned by DRInverter1 }\end{array}$}} \\
\hline & & & \\
\hline \multicolumn{4}{|c|}{ Inverter2Bidder owned by DRInverter2 } \\
\hline \multicolumn{4}{|c|}{ Inverter1Bidder from DRInverter1 is participating } \\
\hline \multirow{2}{*}{\multicolumn{4}{|c|}{$\begin{array}{l}\text { GeneratorBidder from DRGenerator is participating } \\
\text { Inverter2Bidder from DRInverter2 is participating }\end{array}$}} \\
\hline & & & \\
\hline \multicolumn{4}{|c|}{ Excess Supply. Next round starting at: $\$ 0.31000003$} \\
\hline \multicolumn{4}{|c|}{ Excess Supply. Next round starting at: $\$ 0.30000004$} \\
\hline \multicolumn{4}{|c|}{ Excess Supply. Next round starting at: $\$ 0.29000005$} \\
\hline \multicolumn{4}{|c|}{ Excess Supply. Next round starting at $\$ 0.28000006$} \\
\hline \multicolumn{4}{|c|}{ Excess Supply. Next round starting at: $\$ 0,27000007$} \\
\hline \multicolumn{4}{|c|}{ Excess Supply. Next round starting at: $\$ 0.26000008$} \\
\hline \multicolumn{4}{|c|}{ Excess Supply. Next round starting at: $\$ 0.2500001$} \\
\hline \multicolumn{4}{|c|}{ Excess Supply. Next round starting at: $\$ 0.24000008$} \\
\hline \multicolumn{4}{|c|}{ Excess Supply. Next round starting at $\$ 0.23000008$} \\
\hline \multicolumn{4}{|c|}{ Purchasing $100.0 \mathrm{~W}$ from Inverter2Bidder } \\
\hline \multicolumn{4}{|c|}{ Purchasing $500.0 \mathrm{~W}$ from Inverter1日idder } \\
\hline \multicolumn{4}{|c|}{ Next hour demand is: 600.0} \\
\hline \multicolumn{4}{|c|}{ Next hour supply is: 600.0} \\
\hline $\begin{array}{l}\text { Total cost is: } 138 \\
\text { Auction is closed. }\end{array}$ & 005 & & \\
\hline
\end{tabular}

Figure 10.4: Auction process in $16^{\text {th }}$ hour.

All generation bidder agents participate in the auction since their base prices are lower than the utility base price. The base prices of the generator, inverter1 and inverter2 are $0.24,0.10$ and $0.12 \$ / \mathrm{kWh}$, respectively. If the submitted bid amount of power is higher than the demand, the aggregator agent runs another round by lowering the base price by 1 cent in each turn. At the end of the $9^{\text {th }}$ round, the generator bidding agent drops. According to Table 10.2, the amounts of power that can be bid on inverter1 and inverter2 during the $17^{\text {th }}$ hour are $500 \mathrm{~kW}$ and $100 \mathrm{~kW}$, respectively. Since the total submission meets the 
demand, the auction process ends and the market clearing price is established. Since two generation units qualify at the same time, the market clearing price is the value of the last bid. Upon receiving the qualification information from the aggregator agent, the bidder agents send new power references to the actuator agents. Figure 10.5 shows a snapshot of the correspondence between aggregator agent and the other bidder agents during the auction process for the $17^{\text {th }}$ hour.

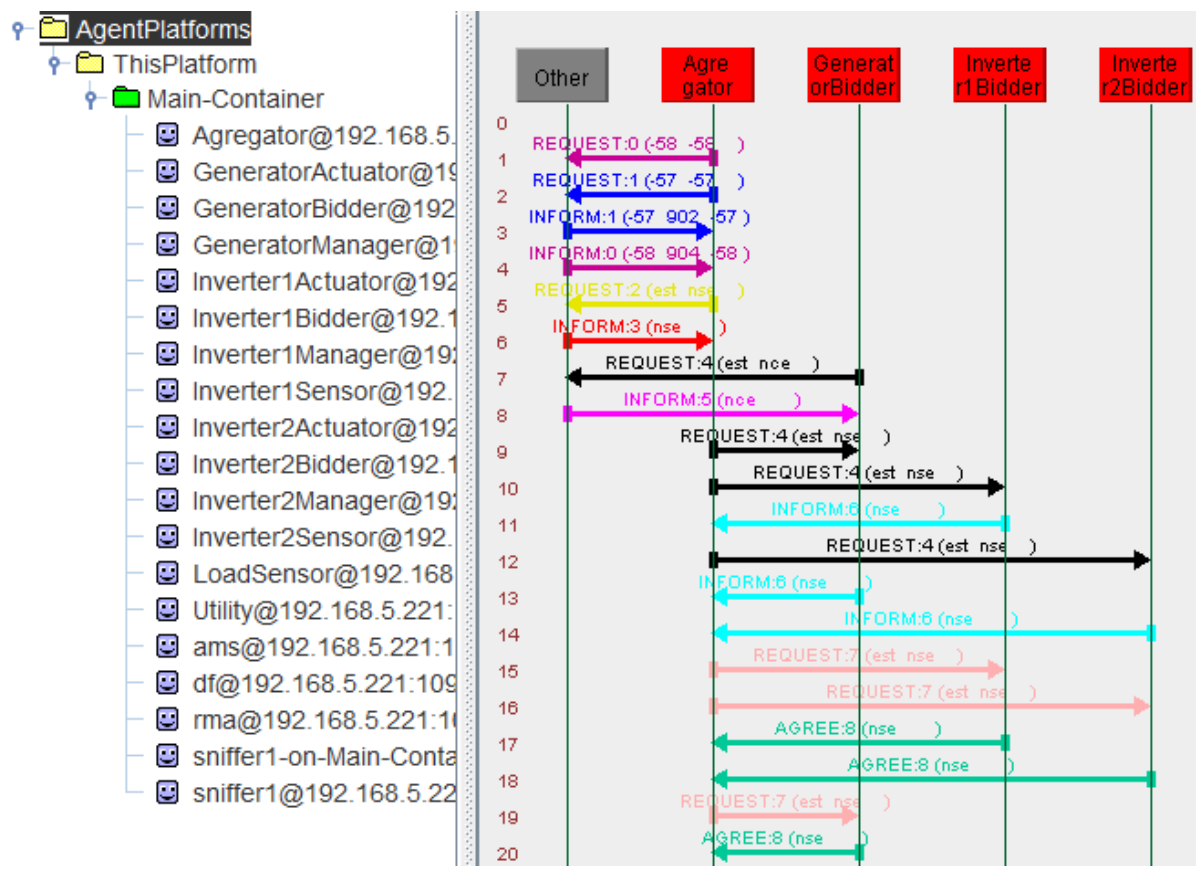

Figure 10.5: Sniffer agent: Aggregator agent interaction with other bidder agents.

Figure 10.6 shows the output power measured in watts by PMU at inverter1 bus. All measurements are collected from the PDC in order to have an exact time reference for measurements. The horizontal numbers on the top of the figures represent the hour of the day. The generation patterns successfully follow the output profile stated in Table 10.2. Figure 10.7 shows the output power measured in watts by the PMU at inverter2 bus. It can be seen from Figure 10.6 and Figure 10.7 that renewable DERs are operating between the $8^{\text {th }}$ and $19^{\text {th }}$ hours when solar irradiance exists. 


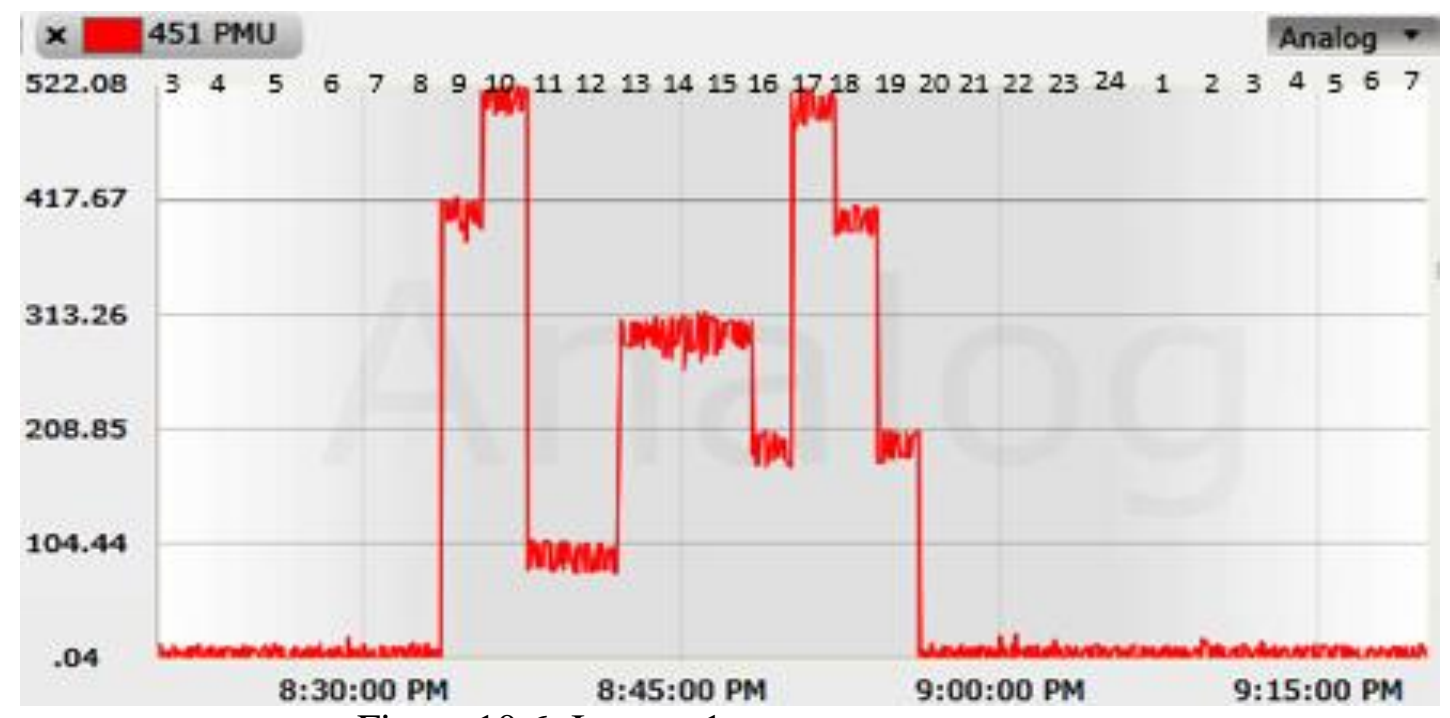

Figure 10.6: Inverter1 power output.

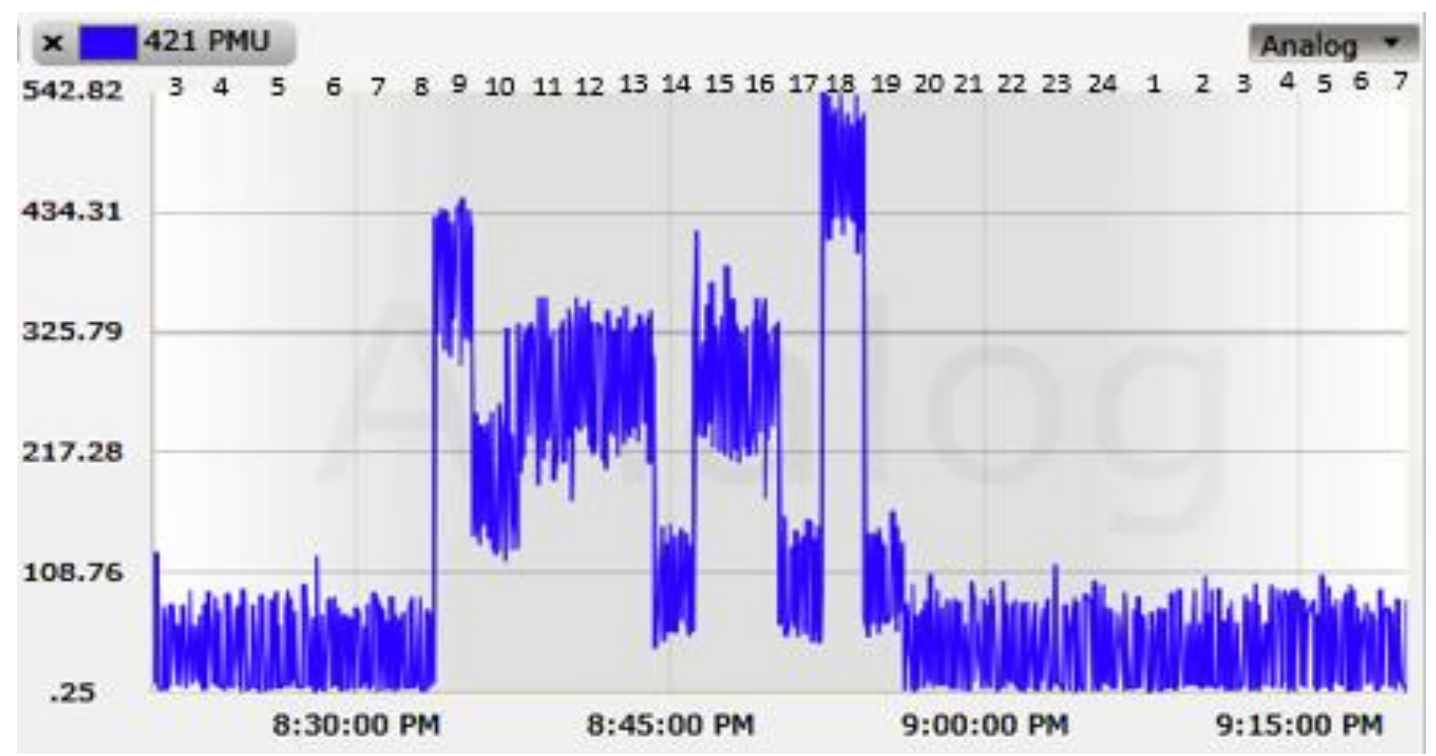

Figure 10.7: Inverter2 power output.

Figure 10.8 shows the power measured at the generator bus. The generator mainly operates at peak hours, where renewable DERs are not available due to the lack of solar irradiation. Figure 10.9 shows the power measured at the utility point of common coupling (PCC). Figure 10.10 shows the voltage profile at utility PCC. Small voltage drops and variations are observed according to power drawn from the utility. 


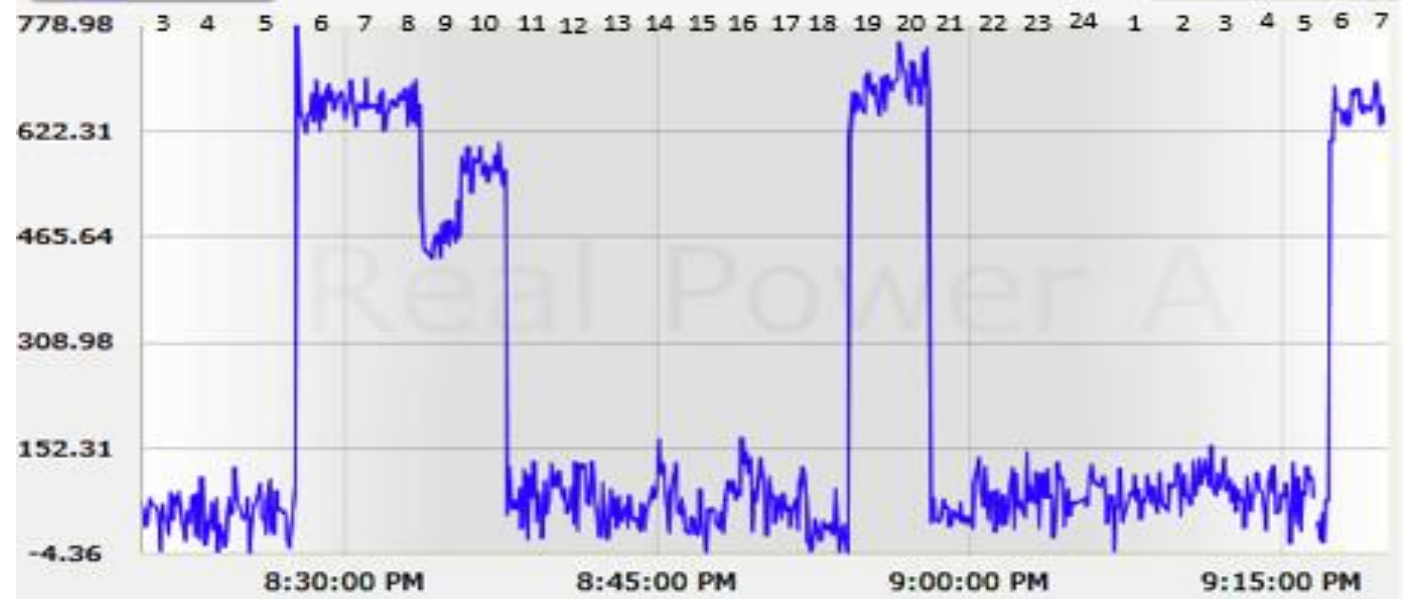

Figure 10.8: Generator power output.

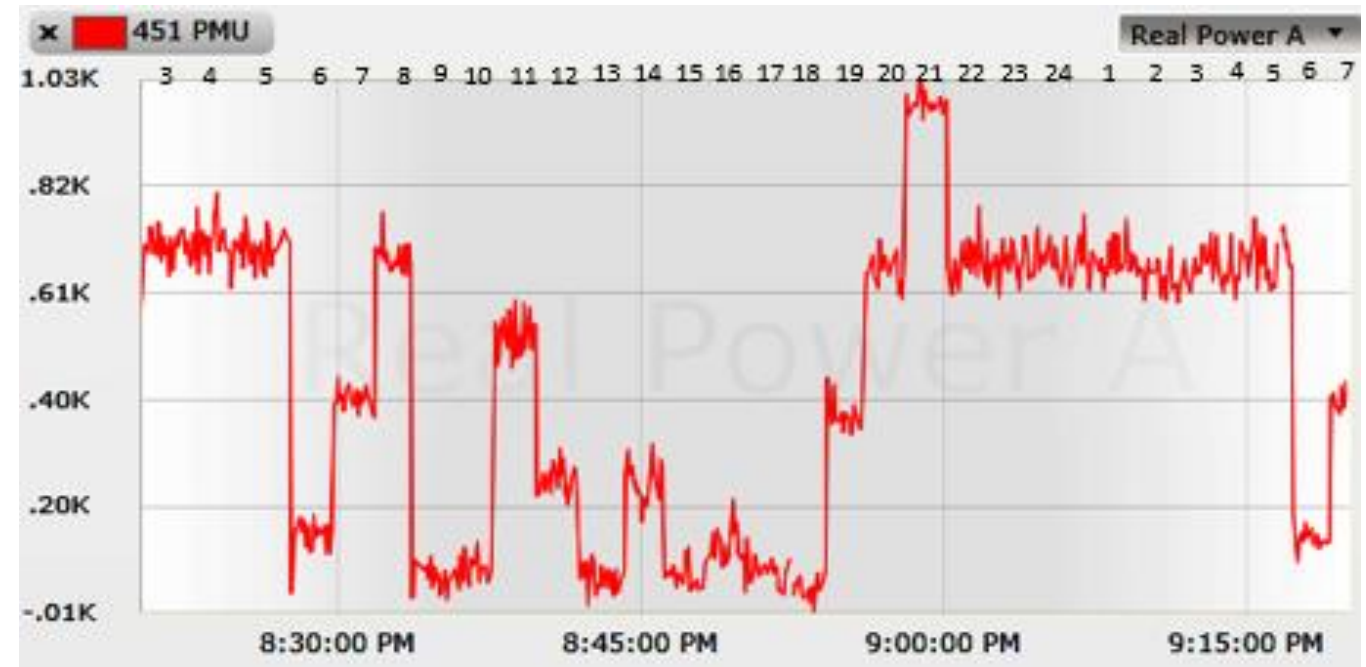

Figure 10.9: Power drawn from utility.

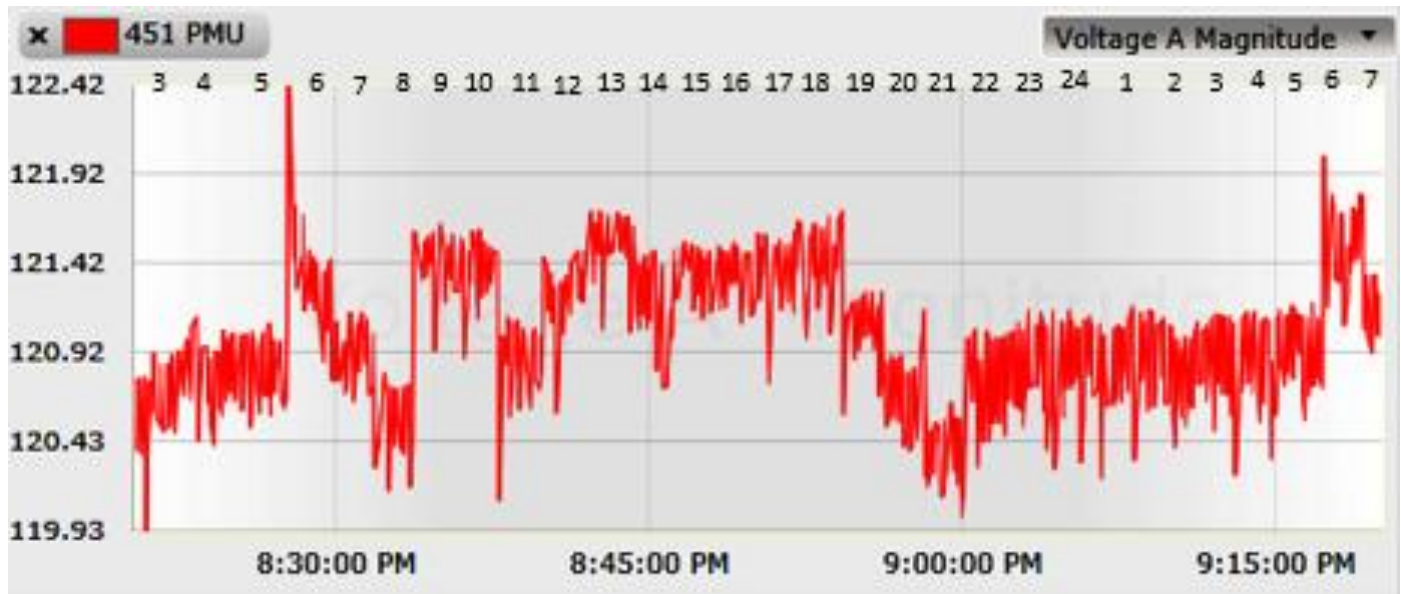

Figure 10.10: Voltage profile at utility point of common coupling. 


\subsection{Conclusion and Summary of the Results}

In this chapter, we proposed a multi-agent based game theory reverse auction model for microgrid market operations featuring conventional and renewable distributed resources. The significance of this study is that a multi-agent based platform is applied to a power system that fully complies with industrial protocols and devices. The results are promising for microgrid field implementations in a larger scale. 


\section{BEHAVIOR MODELING AND AUCTION ARCHITECTURE OF NETWORKED MICROGRIDS FOR WIDE AREA REQUENCY SUPPORT}

\subsection{Introduction}

This chapter investigates realistic behavioral modeling of utility-independent networked microgrid agents under uncertain conditions for power system frequency support. First, we developed a novel behavior modeling of microgrid bidders using a partially observable Markov process (POMDP) to maximize profit of the microgrid operator agents in uncertain market environments. Then, we adopted a reverse auction mechanism for the AGC scheme to enable a competition among microgrid agents for provision of the local area frequency support by a central aggregator controller. Finally, we developed a laboratory-based industrial multi-agent framework for real-time experimental verification of the proposed behavior modeling and auction architecture.

In the developed framework, the auction architecture includes a central aggregator and networked auctioning agents for a provision of local area frequency support in case of a sudden supply/demand imbalance. The POMDP technique is used to determine a shortterm bidding policy of the microgrid agents by online belief updates. To demonstrate the effectiveness and communication architecture of the proposed auction architecture, a realtime laboratory experiment is performed at a Smart Grid Test Bed using a small scale power system including actual generation units, Intelligent Electronic Devices (IEDs) and an industrial multi-agent framework using Open Connectivity Unified Architecture (OPC 
UA) middleware, Foundation for Intelligent Physical Agents (FIPA) and IEC 61850 standards.

The large-scale wind and solar generation-based energy resources show intermittent generation profiles resulting in considerable stochastic frequency variations due to the natural factors, such as wind speed fluctuations and cloudy weather on photovoltaic systems. The traditional electric utility service mechanism is undergoing a change with increased penetration of independent autonomous private microgrids. Conventional frequency control and energy markets are suitably designed for power systems consisting of bulk generation and customer loads. Uncertain generation profiles from renewable resources introduce serious challenges to system operations. New widearea closed-loop power system frequency control and market mechanisms are required to mitigate the negative effects of sudden large generations and demand imbalances.

Some studies in literature have recently addressed the new frequency control techniques in emerging power systems under the smart grid concept. In [153], a new intelligent agent-based Automatic Generation Control (AGC) is proposed to provide a robust probabilistic method of reasoning under uncertainty. The provision of frequency control reserves from aggregated multiple microgrids has been proposed to support ancillary-services in deregulated market mechanisms [154]. Game theoretic [155] and multi-agent [156]-[157] cooperative control methods are studied to enable load-frequency control in power systems. For any kind of control, a competitive market mechanism stimulates the aggregation process.

Auction-based market approaches have proven to be a suitable mechanism in microgrid-based power systems for coordinating seller and buyer entities by the authors 
[158]. Agent-based market and auction models have been implemented to maximize the benefits of generation units by analyzing the effects and evaluation of bidding strategies [159]-[161]. An incentivized auction approach was proposed for demand response management in Vehicle-to-Grid (V2G) systems [162]. The hybrid immune algorithmbased particle swarm optimization [163], game-theoretic approach [164], and the incentive-based approach [165] have been implemented as auction mechanisms. However, the application of a practical auction mechanism for large-scale geographically dispersed and networked microgrids is difficult to put into practice. It is not possible to deploy a system operator in each microgrid, which makes the delegation of the auction process to intelligent agent systems considerably more cost effective. In agent-based auction designs, bilateral spot energy market allows prosumer microgrids to negotiate the price and deliver the energy within seconds. Agent-based systems consistently provide higher profits than their human counterparts, yet these agents still might require occasional human operator support. Intentions and behaviors of the agents should be considered, and self-decision strategies should be modeled to maximize the generated revenue on behalf of microgrid operators. As selfish and greedy agents' preferences are the priority as independent microgrid bidders, a theoretical competitive equilibrium cannot be determined, thus practical belief-based schemes considering behavioral approaches must be adopted.

\subsection{Automatic Generation Control for Intermittent Generation Profile}

Conventional AGC models utilize only bulk generation units to put the system frequency back to its nominal value in response to customer load changes. This load change is inherently stochastic due to the variability of customer behavior. With the current advent 
of large-scale penetration of renewable generation, a new considerable amount of stochastic negative load (intermittent generation) is also introduced. As an example, a twoarea power system with networked microgrid penetration is shown in Figure 11.1.

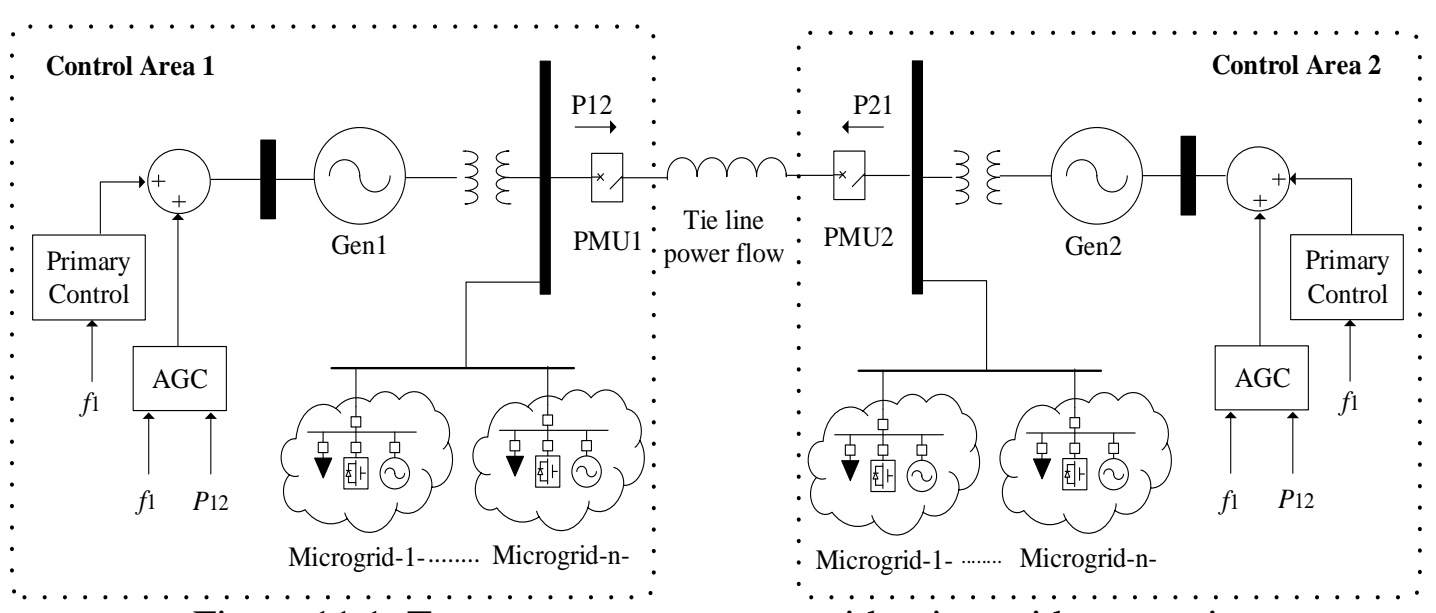

Figure 11.1: Two area power system with microgrid penetration.

In a conventional power system, the updated Area Control Error (ACE) represented by the power flow from tie-line and deviation of the system frequency is as follows:

$$
\begin{gathered}
A C E_{j}=\beta_{j} \Delta f+\Delta P_{t i e_{j}} \\
\Delta P_{g}=-\frac{1}{R_{s y s}} \Delta f
\end{gathered}
$$

where $j=1,2, \ldots, N$ is the control area number, $\Delta$ Ptie is the active power deviation from scheduled power flow, $\beta$ is the frequency bias constant for the control area, $\Delta f$ is the frequency deviation resulting from the free governor operation implemented to respond in proportion to the equivalent of total individual governor droop coefficient Rsys, and $\Delta P g$ is the change in total power generation.

In this chapter, we intend to exploit aggregated use of utility-independent microgrids in a controllable manner to mitigate uncertainty and to support the AGC control. 
Therefore, in addition to stochastic intermittent generation and customer load changes, we introduce a dispatchable term $\triangle P a g g$ to model the aggregated microgrid power, as shown in Figure 11.2.

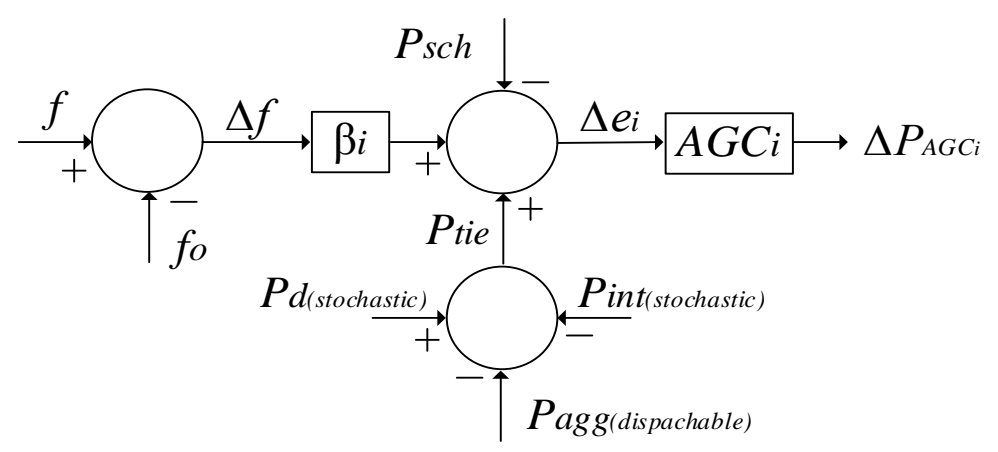

Figure 11.2: AGC control with intermittent generation.

In each AGC cycle, the aggregated power $\triangle$ Pagg is auctioned with frequent updates.

$$
\Delta P_{t i e_{j}}=\left(\Delta P_{d_{j}}-\Delta P_{\text {int }_{j}}-\Delta P_{a g g_{j}}\right)
$$

The power exchange deviation in the control area tie-line $\Delta$ Ptie can be assumed as the sum of bulk load change $\Delta P d$, intermittent generation change $\Delta P$ int, and the aggregated dispatchable microgrid power $\triangle P$ Pagg in the control area. As shown in Figure 11.2, in addition to stochastic bulk load and intermittent generation change, we introduce a deterministic value $\triangle P a g g$ to contribute AGC to converge ACE to zero error rapidly. Psch is the scheduled power flow between neighboring control areas. The main goal of the auctioned aggregated power $\triangle P a g g$ is to minimize the total cost of the frequency provision reserves during AGC. The aggregated power cost should be a cheaper option as a substitution of bulk generation units. The auction process should be dynamically fast, and should update the dispatch power references of the microgrids rapidly. 


\subsection{Wide-Area Auction Mechanism}

The reverse auction model is a good fit for aggregation schemes, where the market environment consists of one buyer and multiple sellers. In this chapter, similar to the wellknown Vickrey auction type, we adopt a second-price reverse auction model to enable microgrid aggregation for AGC support. The Vickrey auction is a partially observable sealed-bid auction type, where bidders submit bids without knowing other the participants' bids. At the same time, the result of the auction has an uncertain outcome, since the agents do not know whether they qualify to be a winner even with the lowest bids.

One of the weaknesses of the common Vickrey auction is that the price discovery is not available to the participant agents. The agents are unsure of their own valuations, which may cause over-bidding. In our proposed reverse auction model, a descending-price mechanism is implemented to come to an equilibrium point. To determine a market clearing price, the price starts with a reserved value of the aggregator agent and continues to fall until the total supply matches the demand. Since the highest market price is initially known, this eliminates the over-bidding weakness. Another weakness is that buyers may use shill bids to mislead the auction participants by trying to introduce bubble generation supply, hence lowering the market clearing price. However, in a second-price Vickrey auction, truthful bidding is the dominant strategy, which lowers this possibility.

\subsubsection{Reserved Price Selection for Aggregator Agent}

Basically, the auctioned aggregated power is a compromise between the cost of AGC-enabled bulk generation units and the aggregated power from dispersed microgrids. The reserved price for the aggregator is the maximum value of the energy price in a particular auction interval that an aggregator agent is willing to pay. Assume a power 
system control area consists of a set $N$ AGC-enabled bulk generation units, where each generation $n \in N$ corresponds to a generation unit. Also, let $L$ refer to the set of tie-line connections, where $l \in L$ is the tie-line of the local area with neighboring control areas. AGC

control can be assumed to be carried out at predefined fixed time periods $(t 0, t 1, \ldots, T)$ by the central aggregator. In each AGC time period, the local generation to be dispatched must be equal to the sum of power flow in tie-lines:

$$
\sum_{n \in N} S_{G n}-\sum_{l \in L} S_{L l}=0
$$

where $S G n$ is the generated active power by each of the $|N|$ units and $S L l$ is the active power flow in each of the $|L|$ tie-lines before AGC is enabled. The cost function of the AGC-enabled generation units is assumed to follow a typical quadratic cost function equation (11.5) where $\mathrm{am}, \mathrm{bm}$, and $\mathrm{cm}$ are corresponding constants:

$$
F_{a g g}\left(P_{G n}\right)=a_{n}+b_{n} P_{G n}+c_{n} P_{G n}{ }^{2}
$$

Equation (11.6) gives the reserved price, which is determined by the well-known economic dispatch algorithm, where PGn is the generation level and Fagg is total generation cost in $\$ / h$.

$$
\min \sum_{n=1}^{N_{G e n}} F_{a g g}\left(P_{G n}\right)
$$

\subsubsection{Reserved Price Selection for Microgrid Agents}

Microgrid operator agents can bid only on dispatchable resources. Therefore, in this study, two types of Distributed Energy Resources (DER) are defined. Renewable resources are assumed to be coupled with storage devices with a grid-tie inverter and the conventional synchronous generators modeled as thermal units. The cost function of a synchronous generator is similar to equation (11.5). However, specifically in microgrids, we assume that 
the fuel/gas price is volatile, hence the generation cost varies in each policy planning stage. Inverter-based storage resources have a simple quadratic function with respect to the current State-of-Charge (SOC) of the battery bank. The cost increases parabolically as the SOC reduces. In this chapter, we assume that the reserved price of a microgrid is the combined function of DERs:

$$
\begin{aligned}
& F_{\text {storage }}\left(\operatorname{SOC}_{\text {Batt }_{i}}\right)=\beta_{i} / \text { SOC }_{\text {Batt }}^{2} \\
& F_{\text {Mic }}=\sum_{i=0}^{N} F_{\text {Gen }}\left(P_{G_{i}}\right)+\sum_{i=0}^{N} F_{\text {Storage }}\left(\text { SOC }_{\text {Batt }_{i}}\right)
\end{aligned}
$$

where SOCBatt is the state-of-charge of each battery bank, $\beta i$ is the corresponding constant, Fstorage is the total generation cost of the battery bank, and Fmic is the microgrid combined cost function.

\subsubsection{Selection of Agent Moods}

The seller microgrid agents try to maximize the benefit of the auction outcome by adding a profit to their reserved price and submitting their bids accordingly. The main objective of this study is to determine how much profit an agent should add on top of the reserved price to maximize the expected future income. The behaviors and belief state are assumed to be moods of the agent that determines how the agent reacts for the next bidding period. The belief state is determined by the past state-action pairs and the observations received after each auction period. Let $S$ represent the set of agent mood states, $A$ is the set of actions that an agent can take in each state and $O$ is the set of observations received as the result of taken action. We assume that an agent in one of the mood states, takes an action according to his optimal policy, and receives an observation whether the agent wins or loses. 


$$
\begin{gathered}
S=\{\text { relax }, \text { greedy }\} \\
A=\{\text { bidRelax, askOperator }, \text { bidGreedy }\} \\
O=\{\text { win, lose }\}
\end{gathered}
$$

Bidrelax corresponds to an action to add a normal profit to the reserved price to maintain the chance to win the auction by submitting a lower price. Oppositely, bidGreedy defines a greedy approach, which attempts to make a considerably higher profit compromising the chance to win the auction. askOperator action corresponds to an agent that is unsure which action to choose, and prefers to ask to a human operator which action to take. POMDP is a machine learning mechanism and an independent microgrid operator would prefer to train the agent with respect to his own beliefs and intuitions. However, the askOperator action probability function can be narrowed down or completely eliminated depending on the planning choice by adjusting reward functions.

Let $I=\{1,2, \ldots, n\}$ be the set of players, and $B i$ is the bidder $i$ 's bid for the item for the agent $i \in I . \Upsilon^{*}$ is the market clearing price after each auction time interval. Due to the agents' behavioral approach, the bid is a function of the mood and reserved price $f:\left(S_{i}, F_{m i c}\right) \rightarrow B_{i}$. The goal of each agent is to maximize the outcome of the auction in each time period $(t 0, t 1, \ldots, T)$. An agent can win the auction and make a profit as long as the bidding price is higher than the resulting market clearing price. Otherwise, for each time it loses, a negative reward signal is received.

$$
\begin{aligned}
& \max \sum_{t=t_{0}}^{T} F_{\text {profit }_{t}}=B_{t}-\tau_{t}^{*} \\
& F_{\text {profit }_{t}}=\left\{\begin{array}{cc}
\text { if }\left(B_{t} \geq \tau_{t}^{*}\right) & B_{t}-\tau_{t}^{*} \\
\text { otherwise } & - \text { Reward }
\end{array}\right\}
\end{aligned}
$$


In this chapter, we defined moods of the agents using the partially observable Markov process (POMDP) to maximize profit of the microgrid operator agents. POMDP provides an efficient mathematical framework for complex planning problems in uncertain and partially observable environments.

\subsection{POMDP and Agent Bidding Policy}

Even though the first applications are in the operational research area, the artificial intelligence community adopted various online and offline POMDP algorithms in the computer science field [166]-[168]. However, they have not received attention in power systems yet. Spoken dialog systems are one of the most successful real-time POMDP implementation areas [167], which shows similarities to the proposed agent-based auction scheme by means of action, state and observations.

In this section, we introduce an implementation concept on how to cast the POMDP framework to the bidding process by determining online short-term plans for the agent. Consider an auction scheme that an agent bids constantly with the same behavior by adding the same profit amount to the reserved price. Its action might not be completely reliable, since the outcome of the auction is not predictable. The volatility of the bulk generation prices and different behaviors of the other microgrid agents' bids introduce considerable uncertainty. The agent does not have any knowledge of how likely it is, it will win the auction with the same continuous behavior. In order to maximize the profits, an agent has to remember the past history of the observations, which are the results of previous auctions, and the state-action pairs, which is usually called the policy. The agent can use this 
information and determine a short-term plan to use in the prevailing new environment to maximize its profits.

In this study, we use the POMDP framework to model this uncertainty problem. In each bidding action, the agent holds a belief from past experience to determine an online short-term policy for its next action, where the success to win the auction is subject to an uncertainty. The belief state is continuously updated based on prior states, actions and observations in each time step. In other words, a belief state from the previous step contributes to the current belief state.

\subsubsection{POMDP Formulation}

POMDP can be formulated as a tuple $<$ S,A,T,R,O,Z, $\gamma, \mathrm{b} 0>$ :

- $\quad S$ is the set of states' $s$ defining agent's world with $s \in S$

- $A$ is the set of actions $a$ that an agent may take $a \in A$

- $\quad T$ defines a transition probability $P\left(s^{\prime} \mid s, a\right)$ which defines the probability of being in $s^{\prime}$ taking action $a$ in state $s$

- $R$ is the expected reward of the state $r(s, a) \in R$

- $O$ is the set of observations an agent receives $o \in O$

- $Z$ is the observation probability $P\left(o^{\prime} \mid s^{\prime}, a\right)$

- $\quad \gamma$ is the discount factor $0<\gamma<1$

- $\quad b 0$ is initial belief

At each time interval, the agent's world is an unobserved state $s$ with a belief state $b$, where $b$ is a distribution over the states. The agent keeps an internal belief state that summarizes its previous experience. The belief state is updated based on the last action taken, current observation and the previous belief space. 
The belief update is defined as a combination of observation function, transition function and current belief. Belief space of an agent is defined as:

$$
B=\left\{b\left(s_{1}\right), \ldots, b\left(s_{s}\right)\right\} \quad b(s) \in \mathcal{R} \quad \forall s \in S
$$

The belief state value cannot be less than zero $b(s) \geq 0$, and the sum of total belief probability of the agent is $\sum_{S} b(s)=1$. Based on $b$, the agent takes action $a$, receives a reward $r(s, a) \in R$, and transitions to an unobserved state $s^{\prime}$, where $s^{\prime}$ depends on $s$ and $a$. The agents receives an observation $o$ ', which depends on $s^{\prime}$ and $a$. At each time interval, $b(s)$ is updated using a state estimator $(S E)$ function. SE computes a new distribution over states $s$ ' given current distribution over states $s$, an action taken $a$, and observation received $O^{\prime}:$

$$
\begin{aligned}
& b^{\prime}\left(s^{\prime}\right)=\mu Z \sum_{s} T b(s) \\
& =\mu P\left(\frac{o^{\prime}}{s^{\prime}}, a\right) Z \sum_{s} P\left(\frac{s^{\prime}}{s}, a\right) b(s)
\end{aligned}
$$

the belief update is a function of observation function $Z$, transition matrix $T, \eta$ is the normalization factor, and current state $b$. Each time interval, the belief state is refreshed with the state estimator:

$$
b_{\tau+1} \leftarrow S E\left(b_{\tau}, a_{\tau}, o_{\tau+1}\right)
$$

$b \tau$ is the belief state at time-step $\tau$, $a \tau$ is the action taken at time $\tau$, and $o \tau+1$ is the observation received as a result of taking action $a \tau$. Each time interval, the agent receives an award $r(s, a) \in R$. The accumulated discounted reward by time $t$ is:

$$
V_{t}=\sum_{\tau=0}^{t-1} \gamma^{\tau} r_{\tau}
$$


The ultimate goal of the agents is to choose proper actions in such a way to maximize the discounted reward by the determined short-term policy, which involves the future actions to be taken.

\subsubsection{Short Term Policy Determination}

Maximizing $V t$ in practice is to find a short-term plan called policy $(\pi)$ defining the actions to take for a time interval. In this study, we assume a 3-step conditional plan as a policy with a 3-step horizon into the future. The number of conditional steps is selected intuitively. It was preferred not to update the policy in each step because it would not be planning towards the future. On the other hand, a late update period would result in outdated results. Selection of the number of conditional plans would result in different consequences in a stochastic environment.

Let $|\mathrm{O}|=2$ be the number of observations that can be received upon taking a set of actions $|\mathrm{A}|=3$. The number of possible 3 -step conditional plans is calculated as: $|A|^{\frac{|O|^{t}-1}{|O|-1}}$. Basically, a 3-step conditional plan is a tree of uniform depth $t$ and constant branching factor $|\mathrm{O}|$, where each node is labelled with an action and each branch designates the observation as a result of taken action. The $\tau$-step conditional plan has a value of $V(s)$, which can be calculated recursively for fixed time intervals $(\tau 0, \tau 1, \ldots, t)$ :

$$
V_{\tau}(s)=r\left(s, a_{\tau}\right)+\gamma \sum_{s^{\prime}} P\left(s^{\prime} / s, a_{\tau}\right) \sum_{o^{\prime}} P\left(o^{\prime} / s^{\prime}, a_{\tau}\right) V_{\tau-1}^{o^{\prime}}\left(s^{\prime}\right)
$$

$a \tau$ is the action associated with the root node of the conditional plan, $V_{\tau-1}^{o^{\prime}}$ is the value of the conditional plan in lower levels of the tree, and $\gamma$ is the discount factor. When running the real-time applications, the agent has incomplete information of state $s$, and maintains a 
belief state $b(s)$. The value of a conditional plan at belief state $B$ is computed as expectations over states:

$$
V(b)=\sum_{s} b(s) V(s)
$$

$B=\{b(s 1), \ldots, b(s s)\}$ is the complete summary of all the actions and observations up to the current time period. For a given initial belief state $b 0$ and history $(a 1, o 1, a 2, o 2, \ldots$, an, on ), there exists a set of t-step conditional plans $N t$ with $n t \in N t,\left\{V_{t}^{n}\right\}$ is set of corresponding values and $\left\{a_{t}^{n}\right\}$ initial actions. The agents' task is to choose the best plan maximizing the $V t$. The value of the best plan at belief state:

$$
V_{N t}^{*}(b)=\max \sum_{s} b(s) V_{t}^{n}(s)
$$

$V_{N t}^{*}(b)$ implies an optimal policy $\pi_{N t}^{*}(b)$. If $N t$ contains all possible conditional plans, then $V_{N t}^{*}(b)$ gives the value of the optimal t-step policy.

\subsection{Implementation Methodology}

To implement the proposed auction mechanism, two types of agents are defined. The central aggregator agent manages the auction process in each AGC time interval and the dispersed microgrid operator agents perform the bidding process. The ultimate goal is to define a decentralized agent-based market mechanism that aggregates utilityindependent microgrids to contribute to AGC frequency regulation with an auction scheme considering the behavioral states of the auctioning agents.

\subsubsection{Central Aggregator}

The central aggregator agent is the main entity that facilitates the auction mechanism. Table 11.1 illustrates the algorithm flow of the auction scheme. 
Table 11.1: Behavioral response-based auction algorithm.

\section{Begin}

Initialize: Aggregator agent initialize the auction, all available microgrids agents subscribe to receive bid solicits;

\section{Loop}

Aggregator agent: calculate $\triangle$ Pagg amount of power to be auctioned for next AGC interval, calculate Fagg reserved price;

Microgrid agent: calculate Fmic reserved price; determine the mood and behavioral-state; calculate $B i$ initial bid value;

Aggregator agent: request bids;

if $(B i<F a g g)$ then

Microgrid agents: submit bid ;

else

Microgrid agents: drop not participating;

end

until (demand => total bid submitted supply) do

Aggregator agent: inform new base price;

Aggregator agent: request new bids;

Microgrid agents: inform submit bids;

\section{end until}

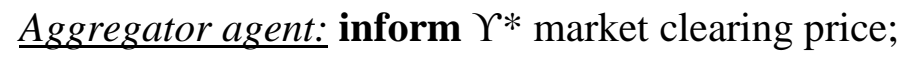

Aggregator agent: inform power references to the qualifiers

\section{Loop end}


In each auction period, the aggregator agent calculates the cost of $\Delta$ Pagg to supply from the bulk generation units as per equations (11.4), (11.5) and (11.6). According to the generation cost function, the reserved price is determined, and the auction is initialized for the next AGC cycle. Then, the central aggregator broadcasts the auction initialization to subscribed agents with the total demand $\triangle P a g g$ and the reserved price Fagg information, and solicits the bids. Receiving the bid request, the microgrid agents calculate their reserved prices and moods. All microgrids submit their bids as per equations (11.8) and (11.10). If the total submitted supply bid amount is higher than the demand, the central aggregator moves to another bidding cycle by lowering the reserved price (\%1 decrement is assumed) and solicits the updated bids. The algorithm continues until some of the microgrid agents' drop from the auction when the price becomes lower than their expected profit. Accordingly, the supply/demand equilibrium is reached and the market clearing price is determined along with the qualifier agents. The resulting power set point references are sent to microgrid operator agents.

\subsubsection{Auctioning Microgrid Agents}

After determining the reserved price for the microgrids as per equation (11.8), the agents select corresponding actions according to their mood behavior state and the threestep conditional plan equation (11.10). The POMDP-based auctioning agent's goal is to maximize the profit by selecting the optimal action for the bidding process. In fact, the goal of the agent is hidden and the states are unobservable, since the result of the auction is unknown until the auction process is over, and a new observation is received.

In this work, the real-time experiments are carried out with three auctioning microgrids. Upon receiving bid requests from the aggregator agent, microgrid agents 
calculate the current reserved price for the next auction period, and runs the online policy update using POMDP algorithm in order to define the action to be taken and the amount of profit to be added on top of the reserved price. The transition function values are shown in Table 11.2, where the state $s$ indicates the agent's goal in each bidding cycle.

Table 11.2: Transition function.

\begin{tabular}{cccc}
\hline \hline Action $(a)$ & State $(s)$ & $\begin{array}{c}\text { Unobserved State } \\
\left(s^{\prime}=\text { relax }\right)\end{array}$ & $\begin{array}{c}\text { Unobserved State } \\
\left(s^{\prime} \text {, greedy }\right)\end{array}$ \\
\hline \multirow{2}{*}{ askOperator } & relax & 1.001 .001 .00 & 0.000 .000 .00 \\
& greedy & 0.000 .000 .00 & 1.001 .001 .00 \\
& relax & 0.700 .500 .70 & 0.300 .500 .30 \\
bidRelax & greedy & 0.700 .500 .70 & 0.300 .500 .30 \\
& relax & 0.700 .50 .70 & 0.300 .500 .30 \\
bidGreedy & greedy & 0.700 .50 .70 & 0.300 .500 .30
\end{tabular}

${ }^{*} P\left(s^{\prime} \mid s, a\right)$ probability distribution for three microgrid agents (MG1, MG $\left.2, ~ M G 3\right)$

Since the agents do not know which selected action will result in positive utility in a stochastic environment, we intuitively selected the probability distribution values to support the decision of agent to maintain same distance between completely relaxed and completely greedy behaviors. The intuitively selected parameters would affect initial conditions of the procedure. As a machine learning algorithm, POMDP mainly improves itself through experience. In this developed framework, we would prefer an experience- 
based learning mechanism rather than a straightforward procedure. This is why a humanin-the-loop mechanism is preferred and suggested in the early learning stages of the agent behaviors. The unobserved state values of the askOperator action are set to 1 in order to ensure the agent's goal remains constant after this action. According to the current belief state, an agent has three available actions to select. It can either ask the microgrid operator which action to choose, or it can autonomously select the bidRelax or bidGreedy action.In the askOperator action, the human operator responds to the agent with an indication as to which action to choose according to its intentions. The auction result (observation) is uncertain, even though the human operator's decision is taken into action and the observation would still be losing the auction subject to a probability. Let's assume that the human operator gives a feedback to take an action as bidRelax, then the probability to win the auction is 0.7 ; and if the action bidGreedy is taken, the probability is relatively lower at 0.6 .

Table 11.3: Observation function.

\begin{tabular}{cccc}
\hline \hline Action $(a)$ & $\begin{array}{c}\text { Unobserved } \\
\text { State }\left(s^{\prime}\right)\end{array}$ & $\begin{array}{c}\text { Observation } \\
\left(\mathrm{o}^{\prime}=\text { WinbidRelax }\right)\end{array}$ & $\begin{array}{c}\text { Observation } \\
\left(\mathrm{o}^{\prime}=\text { WinbidGreedy }\right)\end{array}$ \\
\hline relax & 0.70 & 0.30 \\
bidRelax & greedy & 0.40 & 0.60 \\
& grelax & 0.50 & 0.50 \\
& relax & 0.50 & 0.50 \\
bidGreedy & greedy & 0.50 & 0.50 \\
& & 0.50 & 0.50 \\
\hline \hline$P\left(o^{\prime} \mid s^{\prime}, a\right)$ probability distribution is the same for three of the microgrid agents
\end{tabular}


This means that the human operator has a chance to fail with his experience by 0.3 and 0.4 , respectively, for bidRelax and bidGreedy actions. The probability of the observation function outcome is shown in Table 11.3. After autonomously selecting bidRelax and bidGreedy actions, there is no feedback from the human operator, and this is expressed in the POMDP by removing the conditioning of the observation on actions and states by setting $P\left(o^{\prime} / a, s^{\prime}\right)=P\left(o^{\prime}\right)^{2}$. In Table $11.3, P\left(o^{\prime}\right)$ has been arbitrarily set to 0.5 .

The rewarding mechanism is designed to encourage agents to make more profit by autonomous actions. When an agent wins an auction, the amount of profit it receives is positive. If the agent qualifies in an auction with a greedy behavior, inherently the reward (profit) received is larger than in relaxed mode. When an agent cannot qualify in an auction cycle, it receives a negative reward signal. In a relaxed behavior, the agent prefers to sell energy while maintaining a minimum profit level. In this work, we assume a realistic profit indicator for agents. In the relaxed mode, the agents add $10 \%$ in addition to the reserved price and $30 \%$ in the greedy mode.

In the relaxed mode, the negative reward is defined as only the maintenance cost of the generation units. In the greedy approach, the negative reward is relatively larger since it includes the regret. The regret defines that the agent might have won the auction, if it would have bid with a relaxed behavior. Agents are expected to take their independent actions by reducing the number of feedback requests from the human operator. Hence, the agents are discouraged to frequently ask feedback from the human operator by defining a small negative reward. This negative reward is defined as the labor cost of the human 
operator. The reward function is shown in Table 11.4. The outcome of the 3-step conditional plan for this study is shown in Table 11.5.

Table 11.4: Reward function.

\begin{tabular}{ccc}
\hline \hline Action $(a)$ & State $(s=$ relax $)$ & State $(s=$ greedy $)$ \\
\hline askOperator & $(-)$ operatorCost & $(-)$ operatorCost \\
bidRelax & $(+)$ lowProfit & $(-)$ maintanence \\
bidGreedy & $(-)$ maintanence & \\
& $(-)$ regret & $(+)$ highProfit \\
\hline \hline
\end{tabular}

${ }^{*} r(s \mid a)$ reward mechanism is the same for three of the microgrid agents

Table 11.5: 3-Step conditional plan outcome.

\begin{tabular}{|c|c|c|c|c|c|c|}
\hline \multirow{2}{*}{ Agent } & \multicolumn{3}{|c|}{ Update Belief } & \multicolumn{3}{|c|}{ Action/Observation Pair } \\
\hline & b0 & b1 & $\mathrm{b} 2$ & $1^{\mathrm{st}}$ & $2^{\text {nd }}$ & $3^{\text {rd }}$ \\
\hline \multirow{2}{*}{$M G 1$} & $(0.70)$ & $(0.61)$ & $(0.54)$ & bidRelax & askOperator & askOperator \\
\hline & $(0.30)$ & $(0.39)$ & $(0.46)$ & winBidRelax & winBidRelax & loseBidGreedy \\
\hline \multirow{2}{*}{$M G 2$} & $(0.50)$ & $(0.60)$ & $(0.43)$ & bidRelax & askOperator & askOperator \\
\hline & $(0.50)$ & $(0.40)$ & $(0.56)$ & winBidRelax & loseBidGreedy & winBidRelax \\
\hline \multirow{2}{*}{ MG3 } & $(0.70)$ & $(0.61)$ & $(0.64)$ & askOperator & askOperator & askOperator \\
\hline & $(0.30)$ & $(0.39)$ & $(0.36)$ & loseBidGreedy & winBidRelax & winBidRelax \\
\hline
\end{tabular}




\section{Belief State Monitoring}

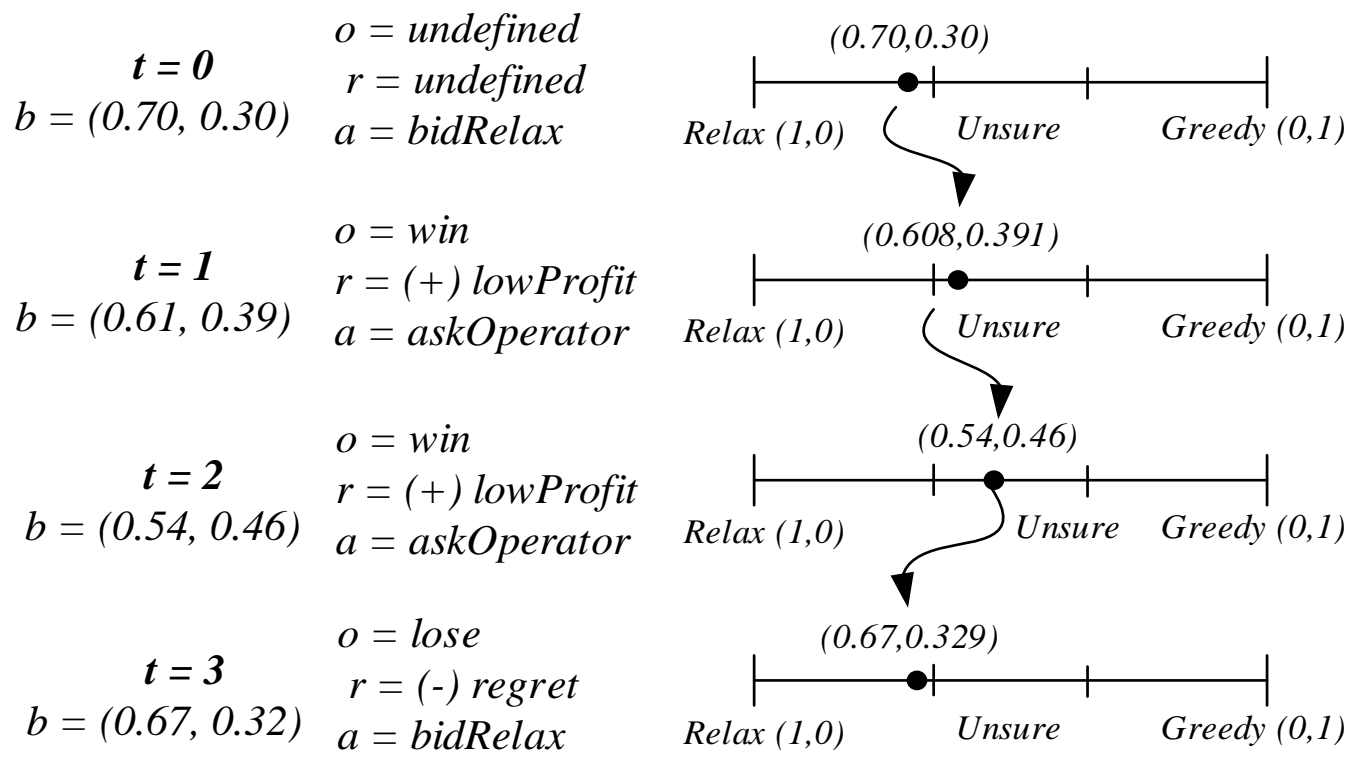

Figure 11.3: Belief monitoring in policy execution for MG1.

For MG1, let the algorithm start at $t=0$, with an initial belief state $b o=(0.70$, 0.30), as shown in the belief monitoring outcome in Figure 11.3. The belief space of an agent has two elements, $b(s=$ relax $)$ and $b(s=$ greedy $)$, where each point in this space $b$ represents a distribution over moods. At $t=0$, the belief state is close to the right corner, which means that the agent's mood is more likely to be relaxed than greedy, however the action taken is still subject to a probability. In the initial state, no observation or reward has yet to be received. bidRelax is taken autonomously as the initial action, and the agent transitions to another state.

The agent wins the auction with a bidRelax feedback from the operator and receives a (+) lowprofit reward. At $t=1$, the updated belief state moves toward the central region, where the agent is more likely to ask the microgrid operator which action to take. The agent wins the auction with a bidRelax feedback from the operator and receives a (+) lowprofit 
reward again. After two successive win observations, the belief state moves very close to the bidGreedy region. According to the conditional plan, the agent takes askOperator action again. In the $t=2$ state, the agent takes bidGreedy action with respect to operator feedback. However, this results a lose observation and a high negative regret reward, which transitions the belief state to bidRelax region for $t=3$.

\subsection{Application Results}

The proposed auction architecture is expected to be successfully applicable to realworld power systems. To illustrate the proposed approach in real-time, an experimental study has been performed at the Florida International University, Smart grid Testbed. A simulation study with a higher number of agents has been performed as well.

\subsubsection{Cyber-Physical Multiagent Framework}

This section briefly explains the hardware, software and the data information model of the multi-agent framework. The two-area power system model comprising 3 microgrids is developed, as illustrated in Figure 11.1. IEDs and Phasor Measurement Units (PMU) are located on system buses to enable monitoring and control. The power system frequency and power flow between two areas are monitored with the deployed PMUs at the tie-points of the transmission lines. IEDs using IEC 61850 communication protocol were deployed at the grid connection point of each microgrid. For actual implementation of decentralized control schemes in power systems, it is imperative to link multi-agent objects to distributed industrial control systems, such as PMUs and IEDs. The required interface is established through a combination of interoperable information data and protocols. IEC 61850 extensions of communication systems for distributed energy resources have been recently 
applied. The IEC 61850 communication provides a standardized information exchange for power systems. However, it is not sufficient to define the behavior of the agents. Therefore, semantics of the exchanged messages should be defined using common knowledge representations. The FIPA is an organization which intends to evolve inter-operable agent communications with an Agent Communication Language (ACL). We implemented the agent framework to run the POMDP algorithm in real-time using IEC 61850 and the FIPA standards, as shown in Figure 11.4.

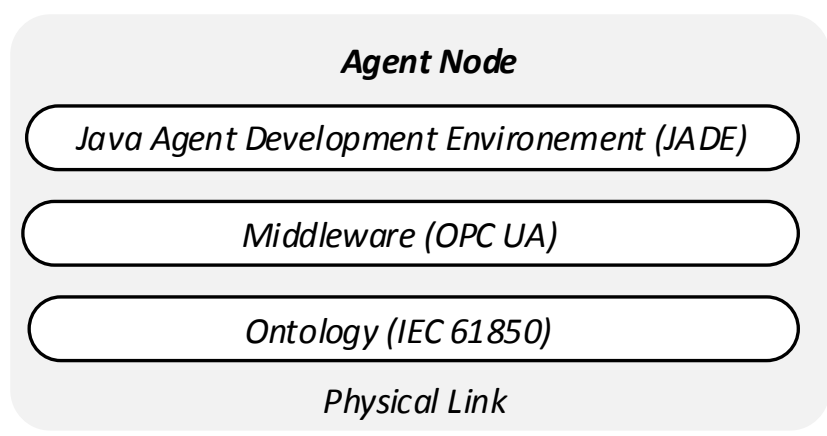

Figure 11.4: Agent node architecture.

ACL messages represent a communicative act or messages (inform, request, refuse etc.) intended to perform some action with precisely defined syntax and semantics. Java agent development framework (JADE) is a software framework to develop agents compliant with FIPA standards with flexible agent behavior methods. Open connectivity unified architecture (OPC UA) middleware is used to connect ACL messages and IEC 61850 logical nodes. OPC UA is a platform-independent specification with a set of industrial standards for system interconnectivity using publish/subscribe mechanism. In this setup, an off-the-shelf OPC UA server is implemented to acquire IEC 61850 logical node measurements. An OPC UA client is embedded in the Java platform to enable JADE to access mapped IEC 61850 node measurements. 


\subsubsection{Real-Time Experimental Demonstration}

The experimental result of the proposed auction architecture was implemented along with the AGC. The experiments are facilitated in a low inertia small-scale power system with actual generators and microgrids. It is desired that an AGC acts slowly and deliberately over tens of seconds of a few minutes.

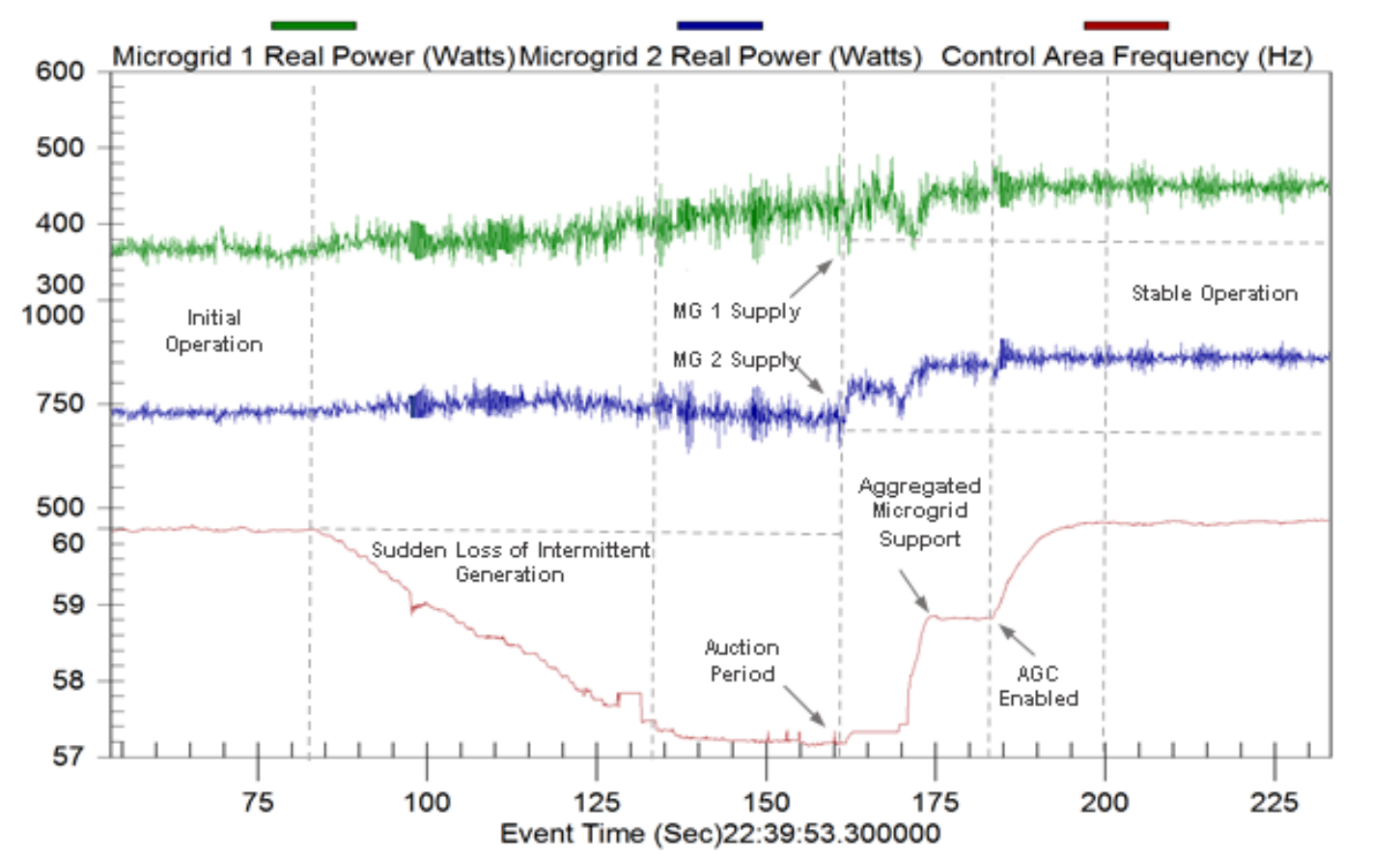

Figure 11.5: Real-time experiment of AGC and auction scheme.

In Figure 11.5, we took a snapshot from PMU measurements over 3 minutes.

Figure 11.5 is based on PMU messages received by a phasor data concentrator in each second. Although the complete snapshot is 3 minutes long, the auction lasts less than 10 seconds. Figure 11.5 starts at the $55^{\text {th }}$ time interval and ends at the $235^{\text {th }}$. The 180 time interval corresponds to 180 seconds in total, which counts each interval as a second. The auction period was kept at approximately 10 intervals corresponding to 10 seconds. In fact, 
the agent communication is almost instant and we adjusted each agent's message exchange in the JADE platform as 1 millisecond. The 10-second delay results from communication delays between agent channels through OPC UA middleware and the generators' response time. As shown Figure 11.5, in the $183^{\text {th }}$ to $196^{\text {th }}$ time interval, generators' response is delayed due to the PI controllers. However, in this study, the frequency drop and the time interval are for laboratory demonstration purposes only. Time intervals can be easily shortened since the agent communication is satisfactorily fast.

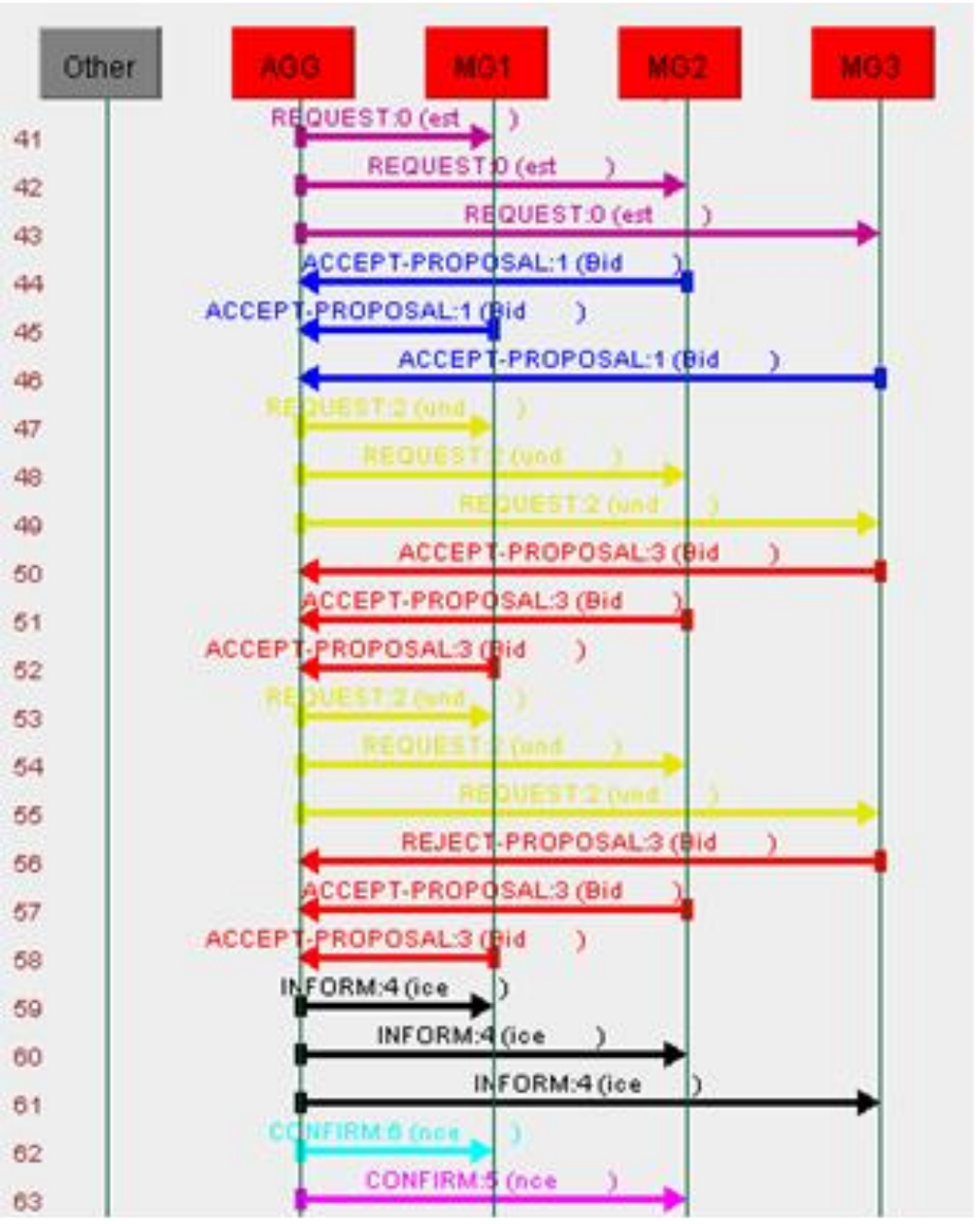

Figure 11.6: Correspondence between central aggregator and microgrid agent. 
Figure 11.6 illustrates correspondence of the aggregator agent and networked microgrid agents in a three-round auction period. In lines 41-43, the aggregator solicits bids from available microgrid agents. As shown in lines 44 to 46, all microgrid agents accept to submit bids. Table 11.6 provides aggregator reserved price and microgrid agent bidding prices, as well as auctioned and bid power amounts.

Table 11.6: Reverse auction result.

\begin{tabular}{ccccc}
\hline \hline Round & $\begin{array}{c}A G G \\
\text { Reserved Price } \\
\text { Auctioned Power }\end{array}$ & $\begin{array}{c}\text { MG1 } \\
\text { Reserved Price } \\
\text { Bided Power }\end{array}$ & $\begin{array}{c}M G 2 \\
\text { Reserved Price } \\
\text { Bided Power }\end{array}$ & $\begin{array}{c}\text { MG3 } \\
\text { Reserved Price } \\
\text { Bided Power }\end{array}$ \\
\hline $1^{\text {st }}$ Round & $0.18 \$$ & $0.13915 \$$ & $0.12892 \$$ & $0.16107 \$$ \\
$2^{\text {nd }}$ Round & $250 \mathrm{~W}$ & $100 \mathrm{~W}$ & $100 \mathrm{~W}$ & $100 \mathrm{~W}$ \\
& $0.17 \$$ & $0.13915 \$$ & $0.12892 \$$ & $0.16107 \$$ \\
$3^{\text {rd }}$ Round & $250 \mathrm{~W}$ & $100 \mathrm{~W}$ & $100 \mathrm{~W}$ & $100 \mathrm{~W}$ \\
& $0.16 \$$ & $0.13915 \$$ & $0.12892 \$$ & Drops \\
\hline \hline
\end{tabular}

${ }^{*}$ Market clearing price is determined as second highest bid $0.12892 \$$.

The negations continue until MG3's bid price is higher than aggregator's reserved price, rejects the proposal, and drops from the auction for that period. In lines 59 to 61 , the market clearing price is informed to all participating agents. In lines 62 and 63, the aggregator agent sends confirm messages to qualifier agents along with the power references. In this case, only MG1 and MG2 covers a portion of the deficit power in the control area. The rest is covered by AGC provided by bulk generations.

The system is in stable operation until the $83^{\text {th }}$ time interval. At the $83^{\text {th }}$, a sudden high amount of intermittent generation loss is introduced with solar panel emulators. The 
control area starts to receive a high amount of power from the neighboring area with respect to the droop parameters. In both control areas, the frequency settles to a lower value in response to droop control of bulk generators. At the $161^{\text {th }}$ time interval, the auction is initiated upon determining the deficit power, and the resulting power references are sent to microgrids. At the $170^{\text {th }}$, microgrids start to deliver resulting power to the grid. This support results a considerable frequency recovery until the $175^{\text {th }}$. Finally, at the $183^{\text {th }}$, AGC is enabled by a local area controller to perfectly mitigate residual frequency error and put the system frequency back to the nominal value. At the end of the $195^{\text {th }}$ time interval, the system frequency is stable and back to the nominal value.

\subsubsection{Simulation Results}

The auction and AGC scheme is demonstrated in a simulation environment with a larger number of microgrid agents. The same two area control in Figure 11.1 is modelled with a penetration of 30 microgrid participants in a simulation environment to demonstrate the effectiveness of the method in a larger scale. Matlab OPC toolbox is used to link JADE agents and SimPowerSystems microgrid objects. Figure 11.7 illustrates the simulation result of the AGC and auction scheme. A sudden loss of intermittent generation is applied at the $3^{\text {rd }}$ simulation time interval and the droop response of the generators take place until the $4^{\text {th }}$ time interval. The agent negotiations take place immediately, and the power amount of ACE is aggregated through qualified microgrids. The microgrids completed frequency support between $4^{\text {th }}$ and the $6^{\text {th }}$ time intervals. At the $6^{\text {th }}$ time interval, the conventional AGC is enabled to put the system frequency back to the nominal value. 


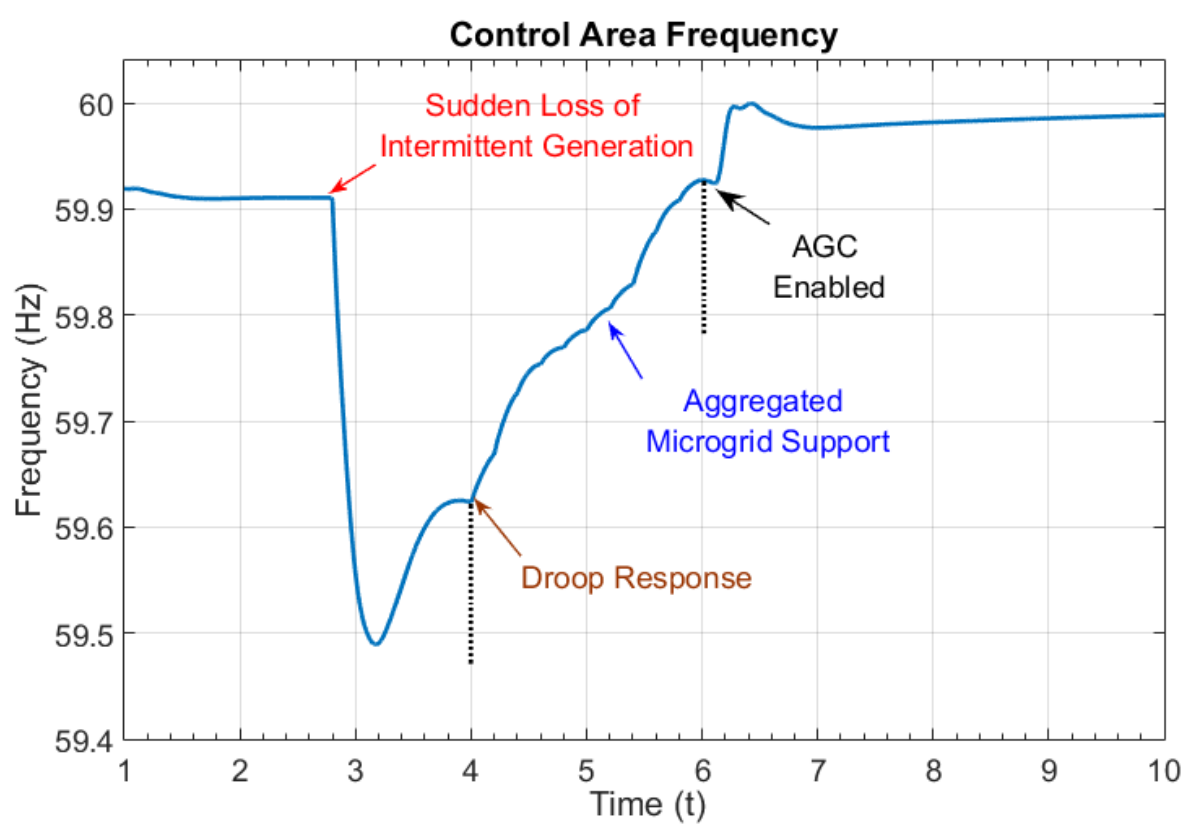

Figure 11.7: Simulation results of AGC and auction scheme.

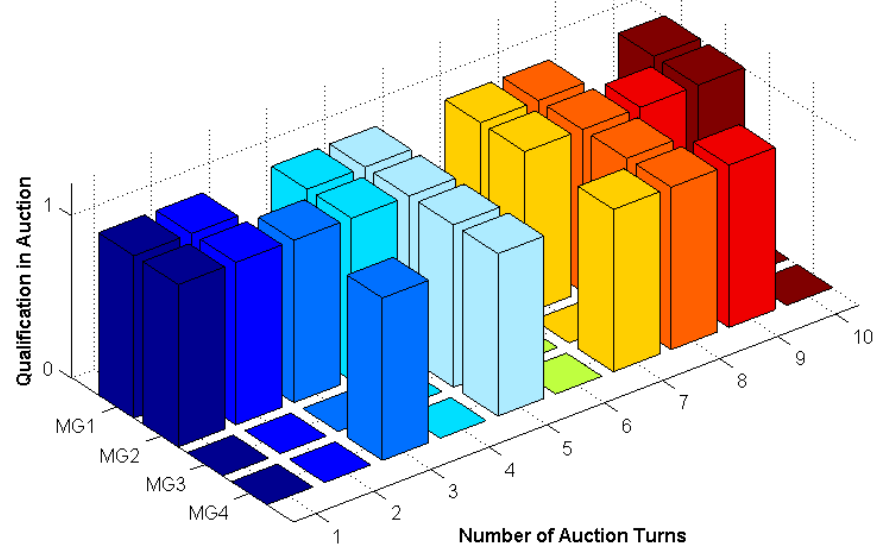

Figure 11.8: Rate of qualification.

A cost comparison analysis was made between agents to demonstrate POMDP leads to maximum profit possible. Among 30 microgrid participant agents, belief monitoring and conditional planning was implemented only to MG1. MG2 and MG3 were kept, bidRelax and bidGreedy, respectively. The rest of the agents, MG4 for instance, constantly bid according to raw cost function value. After training the MG1 agent over a 
period of some time, a comparative analysis on qualification rate and generated revenue was obtained for a 10-term auction. Generation constraints are assumed for all agents for a sound competition. The comparison between MG1, MG2, MG3, and MG4 is given in Figure 11.8 and Table 11.7. As shown in Figure 11.8, bidRelax behavior agent, is the most qualified among all agents due to its constant low value bidding behavior. On the other hand, the bidGreedy behavior agent fails to qualify most of the time. The MG1 agent was able to qualify with reasonable bidding values. Although the bidRelax behavior agent succeeded to qualify more auctions with a greater number of rate, MG1 agent generated more revenue compared to all other agents.

Table 11.7: Generated revenue comparison.

\begin{tabular}{cccc}
\hline \hline MG1 & MG2 & MG3 & MG4 \\
\hline $1.10866 \$$ & $1.09327 \$$ & $0.41517 \$$ & $0.76657 \$$ \\
& & & \\
\%80 Success & \%90 Success & \%20 Success & \%50 Success \\
Rate & Rate & Rate & Rate
\end{tabular}

\subsection{Summary of the Results and Conclusion}

Auction-based aggregation of networked utility-independent microgrids was discussed. A POMDP-based probabilistic planning algorithm was proposed to model intelligent agent behaviors during the auction process. Realistic bidding behaviors were modeled considering the individual profit maximization, rather than global welfare, as in the real-world. It has been shown that the proposed auction architecture can provide a useful mechanism to increase power system resiliency in the presence of high intermittent generation penetration by supporting the power system frequency along with the conventional AGC. The proposed auction mechanism was applied to a laboratory-based 
power system, including actual generators, load models and inverter-based solar generation emulators. An industrial-based multi-agent framework and a communication platform was introduced using FIPA, IEC 61850 and OPC UA standards. It has been presented that the proposed agent-based market mechanism can be successfully implemented in actual power systems. 


\section{CONCLUSIONS AND RECOMMENDATION FOR FUTURE WORKS}

\subsection{Conclusions}

This dissertation presented new techniques for the control, energy management, and protection of wide-area power systems and active distribution networks to improve the performance of the smart grid in terms of efficiency, reliability, communication, aggregation and human/agent participation. Four laboratory-scale hardware/softwarebased test platforms were developed to experimentally evaluate the contributions of this dissertation. The first platform consists of synchrophasors for wide-area and microgrid power system monitoring and control. As second platform, an IED-based protection test platform was built to investigate agent-based communication-assisted protection schemes. This platform is equipped with 3 non-directional and 2 directional feeder protection IEDs. The third test platform is the multi-agent interoperability testbed featuring FIPA, OPC UA and IEC 61850 data communication protocols. The final test setup constructs the wireless communication backbone using hybrid mesh network protocol and Internet-based cloud communication infrastructure. All developed setups have a real-time control and monitoring capabilities, which make the systems unique platforms for enhanced power system studies and performance analysis, such as efficiency, distributed control, distributed communication and distributed protection.

The involvement of synchrophasors in microgrids was investigated for various operation and monitoring applications. We demonstrated how synchrophasors can contribute to microgrid automation applications. Protection event analysis, islanding, 
resynchronization and remote generation dispatch experiments were realistically demonstrated. The results show that the time-synchronized phasor measurements are ubiquitous for fast and comparable data acquisition for future smart grid operations. Moreover, this study shows how synchrophasor measurements can be used to capture and record different events for post-mortem analysis.

A coordinated power flow control method of multi power electronic devices was introduced for a hybrid AC/DC microgrid operated in both grid-connected and islanded modes. The microgrid has PV modules and a synchronous generator that supply energy to its DC and AC sides. Battery banks were connected to the DC bus through bi-directional DC/DC converters. The AC side and DC side were linked by the bi-directional AC/DC inverter. The system topology and control algorithms were tested with the influence of pulse loads and renewable energy output power variations. The simulation and experimental results demonstrated that the microgrid with the developed algorithm can greatly increase the system stability and robustness.

A new agent-based protection scheme of autonomous microgrids was introduced using the industrial distributed communication IEC 61850 GOOSE protocol. Overcurrent and frequency protection challenges were assessed. A practical over current selectivity mechanism was introduced based on feeder characteristics and the location of the IEDs considering RAS. An online adaptive frequency selectivity approach was proposed to avoid pulse load effects in low-inertia autonomous microgrids. A state-of-the-art cyberphysical microgrid setup was introduced including IEDs and actual power system components. Experimental results were demonstrated in order to validate the effectiveness of the proposed schemes. The results were found to be promising for actual field 
deployment of the challenging protection selectivity and RAS schemes in low-inertia autonomous microgrids.

Cutting-edge communication technologies and advanced distributed algorithms play critical role on futuristic smart grid solutions, and protection schemes in particular due to high-speed clearing requirement. A new distributed protection approach was introduced for microgrids with communication constraints. First, a consensus-based auction algorithm was presented for fault localization. Then, a DDFS-based network topology adjustment algorithm was demonstrated. We successfully demonstrated the feasibility of microgrid protection in a fully distributed manner, even with communication constraints. The proposed algorithm was immune to the high impedance fault detection problem, since only a simple pick-up current threshold selection is required for all IEDs. Furthermore, this method completely eliminates the time delay coordination, which has been traditionally used in conventional protection for decades.

For wide-area aggregation of multi-microgrids, an auction-based aggregation of networked utility-independent microgrids was discussed. A POMDP-based probabilistic planning algorithm was proposed to model intelligent agent behaviors during the auction process. Realistic bidding behaviors were modeled considering individual profit maximization, rather than global welfare, as in the real-world. It has been shown that the proposed auction architecture can provide a useful mechanism to increase power system resiliency in the presence of high intermittent generation penetration by supporting the power system frequency along with the conventional AGC. 


\subsection{Future Work}

In this subsection, the new trends on smart grid were highlighted and open research areas were discussed. Comparing the earlier and the current research works, we identified the following trends:

Communication-dependent power grid: Modern sensor networks stimulate the flexibility and application range for wide area implementation of smart grids. Wired communication technologies for distributed control applications, such as market negotiations, power sharing, aggregation, and protection, may not be adequate. Therefore, the smart grid requires an extensive wireless communication research effort including new protocols and enhancing the existing ones. To conduct wireless communication experiments comparable to a real-world environment, there is a significant need for smart grid-oriented wireless communication research. This wireless communication backbone would allow more dynamical topology changes and field deployments. Furthermore, wireless communication testbeds should initiate outdoor deployments, as well as laboratory-scale indoor applications.

Security \& privacy awareness: Any research domain in smart grids should be accompanied with a cyber-physical interface. Along with the targeted research area, related security concepts are inherently involved. Any achieved research success without a specific security and privacy consideration can be assumed as an evolving work. Thus far, many security studies focused on intrusion detection and attack demonstrations. However, to achieve the future goals and provide a reliable smart grid network, further countermeasure techniques and algorithms should be developed and verified in cyber-physical smart grid 
testbeds. Online and offline forensic analysis research should be encouraged. Encryption and certificate methods need to be studied.

Software Defined Networks: The recently emerged software defined networking (SDN) paradigm can perfectly address resilient and secure data collection challenges by splitting controls of networks and data flow operations. The major goal of SDN is to interact with the switches, and thus create an open networking architecture for everyone. In this way, one can achieve a global view of the entire network and make global changes without having to access each device's unique hardware. Therefore, various large-scale network architectures can be deployed and maintained with ease while still featuring resiliency and robustness. The data communication of the smart grid necessitates upgrading the existing infrastructure with various components. SDN-enabled devices would offer a resilient communication infrastructure in all smart grid domains.

Data Interoperability: Interoperability is the ability of two or more devices to exchange information and work together in a system. This is achieved using published objects and data definitions, standard commands and protocols. The smart grid interoperability requirements are clear. All stakeholders need to use commonly agreedupon compatible data exchange formats for a fully integrated framework. The power system framework must work together, which are not just across the technical domains of the smart grid, but also across stakeholder communities in enterprises not part of the existing utility industry. The future challenges include expanding existing protocols and providing ongoing definitions of information models and information exchange requirements. Web-based semantic interoperability frameworks should be investigated further. 
Distributed control: Centralized control methods of operation require a highperformance central processing unit and are more susceptible to single-point failures, where managing the vast amount of data generated from the extensive deployment of smart devices becomes infeasible. In contrast to centralized control, the emerging smart grid concept is in a trend to adopt distributed methods as a result of the highly dynamic behavior of the power grid. Distributed control approaches intend to provide autonomy for different control layers by enabling an event-driven peer-to-peer communication structure, where central control schemes mainly rely on master-slave interactions. In power system applications, the implementation of distributed control is established using multi-agent frameworks, which are composed of interacting multiple intelligent agents to achieve a global or a local objective function. The number of testbeds implementing real-time agentbased control schemes need to be increased.

Interconnection of testbeds: Most institutions may not afford to construct multiple research area testbeds with various hardware and software environment conditions to carry out experimental research. There is a trend to form testbed federations to merge testbeds with different capabilities. Establishing interconnected testbeds is not a simple task. It also necessitates university or institution-wide management and approval. Furthermore, power system applications are considered as high safety risk experiments. However, the interconnection of individual testbeds can be realized in several forms, such as using virtual private networks (VPN) and cloud-based communication via the Internet. Realization of a combination of virtual components is a non-trivial challenge and should be supported with a common API for all participants. Interoperability and standard communication protocols can be a problem solver in such circumstances. 


\section{REFERENCES}

[1] X. Fang, S. Misra, G. Xue and D. Yang, "Smart Grid - The New and Improved Power Grid: A Survey," in IEEE Communications Surveys \& Tutorials, vol. 14, no. 4, pp. 944980, Fourth Quarter 2012.

[2] Hernandez, Luis, Carlos Baladron, Javier M. Aguiar, Belén Carro, Antonio J. SanchezEsguevillas, Jaime Lloret, and Joaquim Massana. "A survey on electric power demand forecasting: Future trends in smart grids, microgrids and smart buildings." Communications Surveys \& Tutorials, IEEE 16, no. 3 (2014): 1460-1495.

[3] Wang, Wenye, Yi Xu, and Mohit Khanna. "A survey on the communication architectures in smart grid." Computer Networks 55, no. 15 (2011): 3604-3629.

[4] Saputro, Nico, Kemal Akkaya, and Suleyman Uludag. "A survey of routing protocols for smart grid communications." Computer Networks 56, no. 11 (2012): 2742-2771.

[5] Yan, Ye, Yi Qian, Hamid Sharif, and David Tipper. "A survey on smart grid communication infrastructures: Motivations, requirements and challenges."Communications Surveys \& Tutorials, IEEE 15, no. 1 (2013): 5-20.

[6] Gungor, V. Cagri, Dilan Sahin, Taskin Kocak, Salih Ergut, Concettina Buccella, Carlo Cecati, and Gerhard P. Hancke. "A survey on smart grid potential applications and communication requirements." Industrial Informatics, IEEE Transactions on 9, no. 1 (2013): 28-42.

[7] Wencong Su; Eichi, H.; Wente Zeng; Mo-Yuen Chow, "A Survey on the Electrification of Transportation in a Smart Grid Environment," in Industrial Informatics, IEEE Transactions on , vol.8, no.1, pp.1-10, Feb. 2012

[8] Grijalva S, Tariq MU. Prosumer-based smart grid architecture enables a flat, sustainable electricity industry. InInnovative Smart Grid Technologies (ISGT), 2011 IEEE PES 2011 Jan 17 (pp. 1-6). IEEE.

[9] Grijalva, Santiago, Mitch Costley, and Nathan Ainsworth. "Prosumer-based control architecture for the future electricity grid." Control Applications (CCA), 2011 IEEE International Con-ference on. IEEE, 2011

[10] Chia-Han Yang; Zhabelova, G.; Chen-Wei Yang; Vyatkin, V., "Cosimulation Environment for Event-Driven Distributed Controls of Smart Grid," in Industrial Informatics, IEEE Transactions on, vol.9, no.3, pp.1423-1435, Aug. 2013.

[11] Locke, Gary, and Patrick D. Gallagher. "NIST framework and roadmap for smart grid interoperability standards, release 1.0." National Institute of Standards and Technology (2010): 33 . 
[12] Amin SM, Wollenberg BF. Toward a smart grid: power delivery for the 21st century. Power and Energy Magazine, IEEE. 2005 Sep;3(5):34-41.

[13] Rahimi F, Ipakchi A. Demand response as a market resource under the smart grid paradigm. Smart Grid, IEEE Transactions on. 2010 Jun;1(1):82-8.

[14] Rahimi F, Ipakchi A. Overview of demand response under the smart grid and market paradigms. InInnovative Smart Grid Technologies (ISGT), 20102010 Jan 19 (pp. 1-7). IEEE.

[15] Andersson G, Donalek P, Farmer R, Hatziargyriou N, Kamwa I, Kundur P, Martins N, Paserba J, Pourbeik P, Sanchez-Gasca J, Schulz R. Causes of the 2003 major grid blackouts in North America and Europe, and recommended means to improve system dynamic performance. Power Systems, IEEE Transactions on. 2005 Nov;20(4):1922-8.

[16] Strategy, NETL Modern Grid. "Advanced metering infrastructure." US Department of Energy Office of Electricity and Energy Reliability (2008).

[17] Karnouskos S, Terzidis O, Karnouskos P. An advanced metering infrastructure for future energy networks. InNew Technologies, Mobility and Security 2007 Jan 1 (pp. 597-606). Springer Netherlands.

[18] Das, R.; Madani, V.; Aminifar, F.; McDonald, J.; Venkata, S.S.; Novosel, D.; Bose, A.; Shahidehpour, M., "Distribution Automation Strategies: Evolution of Technologies and the Business Case," in Smart Grid, IEEE Transactions on , vol.6, no.4, pp.2166-2175, July 2015

[19] Cintuglu MH, Elsayed AT, Mohammed OA. Microgrid automation assisted by synchrophasors. InInnovative Smart Grid Technologies Conference (ISGT), 2015 IEEE Power \& Energy Society 2015 Feb 18 (pp. 1-5). IEEE.

[20] Vogt H, Weiss H, Spiess P, Karduck AP. Market-based prosumer participation in the smart grid. InDigital Ecosystems and Technologies (DEST), 2010 4th IEEE International Conference on 2010 Apr 13 (pp. 592-597). IEEE.

[21] Roberts, B.P.; Sandberg, C., "The Role of Energy Storage in Development of Smart Grids," in Proceedings of the IEEE, vol.99, no.6, pp.1139-1144, June 2011

[22] Mohd, A.; Ortjohann, E.; Schmelter, A.; Hamsic, N.; Morton, D., "Challenges in integrating distributed Energy storage systems into future smart grid," in Industrial Electronics, 2008. ISIE 2008. IEEE International Symposium on, vol., no., pp.16271632, June 30 2008-July 22008.

[23] T. Ma and O. A. Mohammed, "Optimal Charging of Plug-in Electric Vehicles for a CarPark Infrastructure," in IEEE Transactions on Industry Applications, vol. 50, no. 4, pp. 2323-2330, July-Aug. 2014. 
[24] T. Ma and O. Mohammed, "Economic analysis of real-time large scale PEVs network power flow control algorithm with the consideration of V2G services," Industry Applications Society Annual Meeting, 2013 IEEE, Lake Buena Vista, FL, 2013, pp. 18 .

[25] Albadi MH, El-Saadany EF. A summary of demand response in electricity markets. Electric power systems research. 2008 Nov 30;78(11):1989-96.

[26] Parikh, Palak P., Mitalkumar G. Kanabar, and Tarlochan S. Sidhu. "Opportunities and challenges of wireless communication technologies for smart grid applications." Power and Energy Society General Meeting, 2010 IEEE. IEEE, 2010.

[27] Gungor, Vehbi C., Bin Lu, and Gerhard P. Hancke. "Opportunities and challenges of wireless sensor networks in smart grid." Industrial Electronics, IEEE Transactions on 57.10 (2010): 3557-3564.

[28] Ericsson, Göran N. "Cyber security and power system communication-essential parts of a smart grid infrastructure." Power Delivery, IEEE Transactions on 25.3 (2010): 15011507.

[29] Mo Y, Kim TH, Brancik K, Dickinson D, Lee H, Perrig A, Sinopoli B. Cyber-physical security of a smart grid infrastructure. Proceedings of the IEEE. 2012 Jan;100(1):195209.

[30] Zaballos, Agustin, Alex Vallejo, and Josep M. Selga. "Heterogeneous communication architecture for the smart grid." Network, IEEE 25.5 (2011): 30-37.

[31] Chowdhury, Sunetra, and Peter Crossley. Microgrids and active distribution networks. The Institution of Engineering and Technology, 2009.

[32] Zechun $\mathrm{Hu}$, Furong Li, "Cost-benefit analyses of active distribution network management, part I: Annual benefit analysis," IEEE Transactions on Smart Grid, vol. 3, pp. 1067-1074, 2012.

[33] Zechun $\mathrm{Hu}$, Furong Li, "Cost-benefit analyses of active distribution network management, part II: Investment reduction analysis," IEEE Transactions on Smart Grid, vol. 3, pp. 1075-1081, 2012.

[34] O. Samuelsson, S. Repo, R. Jessler, J. Aho, M. Karenlampi, A. Malmquist, “Active distribution network - Demonstration project ADINE," in Innovative Smart Grid Technologies (ISGT Europe), 2010 IEEE PES, pp. 1-8.

[35] Martins, Vinicius F., and Carmen LT Borges. "Active distribution network integrated planning incorporating distributed generation and load response uncertainties." Power Systems, IEEE Transactions on 26.4 (2011): 2164-2172. 
[36] Gill, Simon, Ivana Kockar, and Graham Ault. "Dynamic optimal power flow for active distribution networks." IEEE Transactions on Power Systems (2013).

[37] Fan, Zhong, Parag Kulkarni, Sedat Gormus, Costas Efthymiou, Georgios Kalogridis, Mahesh Sooriyabandara, Ziming Zhu, Sangarapillai Lambotharan, and Woon Hau Chin. "Smart grid communications: overview of research challenges, solutions, and standardization activities." Communications Surveys \& Tutorials, IEEE 15, no. 1 (2013): 21-38.

[38] Yan, Ye, Yi Qian, Hamid Sharif, and David Tipper. "A survey on cyber security for smart grid communications." Communications Surveys \& Tutorials, IEEE14, no. 4 (2012): 998-1010.

[39] Liu, Jing, Yang Xiao, Shuhui Li, Wei Liang, and C. L. Chen. "Cyber security and privacy issues in smart grids." Communications Surveys \& Tutorials, IEEE 14, no. 4 (2012): 981-997.

[40] Hong, Junho, Shinn-Shyan Wu, A. Stefano, Ahmed Fshosha, Chen-Ching Liu, Pavel Gladyshev, and Manimaran Govindarasu. "An intrusion and defense testbed in a cyberpower system environment." In IEEE Power and Energy Society General Meeting. 2011.

[41] F. Hu, Q. Hao and K. Bao, "A Survey on Software-Defined Network and OpenFlow: From Concept to Implementation," in IEEE Communications Surveys \& Tutorials, vol. 16, no. 4, pp. 2181-2206, Fourthquarter 2014.

[42] Jianchao Zhang, Boon-Chong Seet, Tek-Tjing Lie and Chuan Heng Foh, "Opportunities for Software-Defined Networking in Smart Grid," Information, Communications and Signal Processing (ICICS) 2013 9th International Conference on, Tainan, 2013, pp. 1-5.

[43] A. Sydney, J. Nutaro, C. Scoglio, D. Gruenbacher and N. Schulz, "Simulative Comparison of Multiprotocol Label Switching and OpenFlow Network Technologies for Transmission Operations," in IEEE Transactions on Smart Grid, vol. 4, no. 2, pp. 763-770, June 2013.

[44] McMorran, A., et al. "Addressing the Challenge of Data Interoperability for Off-Line Analysis of Distribution Networks in the Smart Grid, IEEE PES Transmission and Distribution Conference and Exposition, Orlando." FL. May (2012).

[45] Von Dollen, Don. "Report to NIST on the smart grid interoperability standards roadmap." Electric Power Research Institute (EPRI) and National Institute of Standards and Technology (2009).

[46] IEEE Vision for Smart Grid Communications: 2030 and Beyond," IEEE Vision for Smart Grid Communications: 2030 and Beyond, pp.1,390, May 312013 
[47] Leccese, F., "An overwiev on IEEE Std 2030," Environment and Electri-cal Engineering (EEEIC), 2012 11th International Conference on , vol., no., pp.340,345, 1825 May 2012

[48] Cintuglu, Mehmet Hazar, and Osama Mohammed. "Simulation of digitalized power system using PMU and intelligent control." Industry Applications Society Annual Meeting, 2013 IEEE. IEEE, 2013.

[49] Fenghui Ren; Minjie Zhang; Soetanto, D.; XiaoDong Su, "Conceptual Design of A Multi-Agent System for Interconnected Power Systems Res $\neg$ toration," Power Systems, IEEE Transactions on , vol.27, no.2, pp.732,740, May 2012

[50] Zhabelova, G.; Vyatkin, V., "Multiagent Smart Grid Automation Archi-tecture Based on IEC 61850/61499 Intelligent Logical Nodes," Industrial Electronics, IEEE Transactions on , vol.59, no.5, pp.2351,2362, May 2012

[51] Hui Wan; Li, K.K.; Wong, K.P., "An Adaptive Multiagent Approach to Protection Relay Coordination With Distributed Generators in Industrial Power Distribution System," Industry Applications, IEEE Transactions on , vol.46, no.5, pp.2118,2124, Sept.-Oct. 2010

[52] Apostolov, Alexander. "Multi-agent systems and IEC 61850." Power En $\neg$ gineering Society General Meeting, 2006. IEEE. IEEE, 2006.

[53] Dimeas, A.L.; Hatziargyriou, N.D., "Operation of a Multiagent System for Microgrid Control," Power Systems, IEEE Transactions on, vol.20, no.3, pp.1447,1455, Aug. 2005

[54] Chun-Xia Dou; Bin Liu, "Multi-Agent Based Hierarchical Hybrid Control for Smart Microgrid," Smart Grid, IEEE Transactions on , vol.4, no.2, pp.771,778, June 2013

[55] IEEE Std 1547.4-2011 IEEE Guide for Design , Operation, and Integration of Distributed Resource Island Systems with Electric Power Systems

[56] N. Hatziargyriou, H. Asano, R. Iravani and C. Marnay, "Microgrids," in IEEE Power and Energy Magazine, vol. 5, no. 4, pp. 78-94, July-Aug. 2007.

[57] R. H. Lasseter, "MicroGrids," Power Engineering Society Winter Meeting, 2002. IEEE, 2002, pp. 305-308 vol.1.

[58] Kundur, Prabha. Power system stability and control. Eds. Neal J. Balu, and Mark G. Lauby. Vol. 7. New York: McGraw-hill, 1994.

[59] Senroy, Nilanjan, Gerald T. Heydt, and Vijay Vittal. "Decision tree assisted controlled islanding." Power Systems, IEEE Transactions on 21.4 (2006): 1790-1797.

[60] A.G.Phadke., J.S. Thorp,. "Synchronized Phasor Measurements and Their Applications" 
[61] Mackiewicz, R.E., "Overview of IEC 61850 and Benefits," Power Systems Conference and Exposition, 2006. PSCE '06. 2006 IEEE PES, vol., no., pp.623, 630, Oct. 29 2006Nov.

[62] Communication Networks and Systems in Substations - Part 7-2, Basic Communication Structure for Substation and Feeder Equipment-Abstract Communication Service Interface (ACSI), IEC61850, Int. Electrotech. Committee, 2003.

[63] IEEE Standard for Synchrophasor Measurements for Power Systems, IEEE Standard C37.118.1, 2011.

[64] IEEE Standard for Synchrophasor Data Transfer for Power Systems, IEEE Standard C37.118.2, 2011.

[65] Mazloomzadeh, Ali, Mehmet Hazar Cintuglu, and Osama Mohammed. "Islanding detection using synchronized measurement in smart microgrids."Innovative Smart Grid Technologies Latin America (ISGT LA), 2013 IEEE PES Conference On. IEEE, 2013.

[66] Mazloomzadeh, Ali, Mehmet H. Cintuglu, and Osama A. Mohammed. "Development and evaluation of a laboratory based phasor measurement devices." Innovative Smart Grid Technologies Conference (ISGT), 2015 IEEE Power \& Energy Society. IEEE, 2015.

[67] J. Sexauer, P. Javanbakht, S. Mohaghengi, "Phasor measurement units for the distribution grid: Necessity and benefits," in Innovative Smart Grid Technologies (ISGT), 2013 IEEE PES, pp. 1-6.

[68] Wache, Markus. "Application of phasor measurement units in distribution networks." In Electricity Distribution (CIRED 2013), 22nd International Conference and Exhibition on, pp. 1-4. IET.G.

[69] Sanchez-Ayala, J.R. Aguerc, D. Elizondo, M. Lelic, "Current trends on applications of PMUs in distribution systems," in Innovative Smart Grid Technologies (ISGT), 2013 IEEE PES, pp. 1-6.

[70] Y. Sun, W. Wu, H. Sun, B. Zhang, X. Cheng, S. Qiu, C. Zhang, H. Wu "A simulation and training system for active distribution network"in Innovative Smart Grid Technologies (ISGT Asia), 2012 IEEE PES, pp. 1-6.

[71] D. Feng, C. D. Booth, "Applications of PMUs in power distribution networks distributed generation," in Universities Power Engineering Conference (UPEC), 2011, pp. 1-5.

[72] Xiong Liu; Peng Wang; Poh Chiang Loh, "A Hybrid AC/DC Microgrid and Its Coordination Control," Smart Grid, IEEE Transactions on , vol.2, no.2, pp.278,286, June 2011 
[73] Crider, J.M.; Sudhoff, S.D., "Reducing Impact of Pulsed Power Loads on Microgrid Power Systems," Smart Grid, IEEE Transactions on , vol.1, no.3, pp.270,277, Dec. 2010

[74] Laaksonen, H.J., "Protection Principles for Future Microgrids," Power Electronics, IEEE Transactions on , vol.25, no.12, pp.2910,2918, Dec. 2010

[75] Najy, W.K.A; Zeineldin, H.H.; Woon, W.L., "Optimal Protection Coordination for Microgrids With Grid-Connected and Islanded Capability," Industrial Electronics, IEEE Transactions on , vol.60, no.4, pp.1668,1677, April 2013

[76] Laaksonen, H.; Ishchenko, D.; Oudalov, A, "Adaptive Protection and Microgrid Control Design for Hailuoto Island," Smart Grid, IEEE Transactions on , vol.5, no.3, pp.1486,1493, May 2014

[77] Ustun, T.S.; Ozansoy, C.; Zayegh, A., "Modeling of a Centralized Microgrid Protection System and Distributed Energy Resources According to IEC 61850-7-420," Power Systems, IEEE Transactions on, vol.27, no.3, pp.1560,1567, Aug. 2012

[78] Tomita, Y.; Fukui, C.; Kudo, H.; Koda, J.; Yabe, K., "A cooperative protection system with an agent model," Power Delivery, IEEE Transactions on , vol.13, no.4, pp.1060,1066, Oct 1998

[79] Hui Wan; Li, K.K.; Wong, K.P., "An Adaptive Multiagent Approach to Protection Relay Coordination With Distributed Generators in Industrial Power Distribution System," Industry Applications, IEEE Transactions on , vol.46, no.5, pp.2118,2124, Sept.-Oct. 2010

[80] Zeng Xiangjun; Li, K.K.; Chan, W.L.; Su Sheng, "Multi-agents based protection for distributed generation systems," Electric Utility Deregulation, Restructuring and Power Technologies, 2004. (DRPT 2004). Proceedings of the 2004 IEEE International Conference on , vol.1, no., pp.393,397 Vol.1, 5-8 April 2004

[81] Manickam, A.; Kamalasadan, S.; Edwards, D.; Simmons, S., "A Novel Self-Evolving Intelligent Multiagent Framework for Power System Control and Protection," Systems Journal, IEEE , vol.8, no.4, pp.1086,1095, Dec. 2014

[82] Su Sheng; Li, K.K.; Chan, W.L.; Zeng Xiangjun; Duan Xianzhong, "Agent-based selfhealing protection system," Power Delivery, IEEE Transactions on , vol.21, no.2, pp.610,618, April 2006

[83] Timbus, A.; Larsson, M.; Yuen, C., "Active Management of Distributed Energy Resources Using Standardized Communications and Modern Information Technologies," Industrial Electronics, IEEE Transactions on , vol.56, no.10, pp.4029,4037, Oct. 2009

[84] Davidson, E.M.; McArthur, S.D.J.; Mcdonald, James R; Cumming, T.; Watt, I., "Applying multi-agent system technology in practice: automated management and 
analysis of SCADA and digital fault recorder data," Power Systems, IEEE Transactions on , vol.21, no.2, pp.559,567, May 2006

[85] Apostolov, A, "Multi-agent systems and IEC 61850," Power Engineering Society General Meeting, 2006. IEEE

[86] Zhabelova, G.; Vyatkin, V., "Multiagent Smart Grid Automation Architecture Based on IEC 61850/61499 Intelligent Logical Nodes," Industrial Electronics, IEEE Transactions on , vol.59, no.5, pp.2351,2362, May 2012

[87] Strasser, T.; Andren, F.; Kathan, J.; Cecati, C.; Buccella, C.; Siano, P.; Leitao, P.; Zhabelova, G.; Vyatkin, V.; Vrba, P.; Marik, V., "A Review of Architectures and Concepts for Intelligence in Future Electric Energy Systems," Industrial Electronics, IEEE Transactions on , vol.62, no.4, pp.2424,2438, April 2015

[88] Pala, D.; Tornelli, C.; Proserpio, G., "An adaptive, agent-based protection scheme for radial distribution networks based on IEC 61850 and IEC 61499,"Integration of Renewables into the Distribution Grid, CIRED 2012 Workshop, vol., no., pp.1,4, 29-30 May 2012

[89] Apostolov, A; Vandiver, B., "IEC 61850 GOOSE applications to distribution protection schemes," Protective Relay Engineers, 2011 64th Annual Conference pp.178,184, 11-14 April 2011

[90] Communication Networks and Systems in Substations - Part 7-4, Basic Communication Structure for Substation and Feeder Equipment- Compatible Logical Node Classes and Data Classes, IEC61850, Int. Electrotech. Committee, 2003.

[91] Communication Networks and Systems for Power Utility Automation for Distributed Energy Resources (DER) — Part 7-420, IEC61850, Int. Electrotech. Committee, 2011.

[92] M. H. Cintuglu and O. A. Mohammed, "Multiagent-based decentralized operation of microgrids considering data interoperability," 2015 IEEE International Conference on Smart Grid Communications (SmartGridComm), Miami, FL, USA, 2015, pp. 404-409.

[93] M. H. Cintuglu, H. Martin and O. A. Mohammed, "An intelligent multi agent framework for active distribution networks based on IEC 61850 and FIPA standards," Intelligent System Application to Power Systems (ISAP), 2015 18th International Conference on, Porto, 2015, pp. 1-6.

[94] IEC 61850 Communication networks and systems in substations - Use of IEC 61850 for the communication between substations pt. 90-1 [Online]. Available: http://www.iec.ch

[95] Wen, Jun, et al. "Evolution Pathway Towards Wide Area Monitoring and ProtectionA Real-World Implementation of Centralized RAS System." Smart Grid, IEEE Transactions on 5.3 (2014): 1506-1513. 
[96] IEEE Application Guide for IEEE Std 1547(TM), IEEE Standard for Interconnecting Distributed Resources with Electric Power Systems,"IEEE Std 1547.2-2008, pp.1,217, April 152009

[97] Bronin, Sara C., and Paul McCary. "Peaceful Coexistence: Independent Microgrids are Coming." Public Utilities Fortnightly (2013).

[98] M. A. Zamani, T. S. Sidhu and A. Yazdani, "Investigations Into the Control and Protection of an Existing Distribution Network to Operate as a Microgrid: A Case Study," in IEEE Transactions on Industrial Electronics, vol. 61, no. 4, pp. 1904-1915, April 2014.

[99] G. T. Heydt, "The Next Generation of Power Distribution Systems," in IEEE Transactions on Smart Grid, vol. 1, no. 3, pp. 225-235, Dec. 2010.

[100] H. J. Laaksonen, "Protection Principles for Future Microgrids," in IEEE Transactions on Power Electronics, vol. 25, no. 12, pp. 2910-2918, Dec. 2010.

[101] M. Kezunovic, "Smart Fault Location for Smart Grids," in IEEE Transactions on Smart Grid, vol. 2, no. 1, pp. 11-22, March 2011.

[102] S. Karnouskos, "Cyber-Physical Systems in the SmartGrid," Industrial Informatics (INDIN), 2011 9th IEEE International Conference on, Caparica, Lisbon, 2011, pp. 2023.

[103] Sortomme, Eric, S. S. Venkata, and Joydeep Mitra. "Microgrid protection using communication-assisted digital relays." Power delivery, IEEE transactions on 25.4 (2010): 2789-2796.

[104] U. Orji; C. Schantz; S. B. Leeb; J. L. Kirtley; B. Sievenpiper; K. Gerhard; T. McCoy, "Adaptive Zonal Protection for Ring Microgrids," in IEEE Transactions on Smart Grid , vol.PP, no.99, pp.1-9

[105] Brahma, Sukumar M., and Adly A. Girgis. "Development of adaptive protection scheme for distribution systems with high penetration of distributed generation." Power Delivery, IEEE Transactions on 19.1 (2004): 56-63.

[106] H. Laaksonen, D. Ishchenko and A. Oudalov, "Adaptive Protection and Microgrid Control Design for Hailuoto Island," in IEEE Transactions on Smart Grid, vol. 5, no. 3, pp. 1486-1493, May 2014.

[107] T. S. Ustun, C. Ozansoy and A. Ustun, "Fault current coefficient and time delay assignment for microgrid protection system with central protection unit," in IEEE Transactions on Power Systems, vol. 28, no. 2, pp. 598-606, May 2013.

[108] H. Wan, K. K. Li and K. P. Wong, "An Adaptive Multiagent Approach to Protection Relay Coordination With Distributed Generators in Industrial Power Distribution 
System," inIEEE Transactions on Industry Applications, vol. 46, no. 5, pp. 2118-2124, Sept.-Oct. 2010.

[109] G. Zhabelova and V. Vyatkin, "Multiagent Smart Grid Automation Architecture Based on IEC 61850/61499 Intelligent Logical Nodes," in IEEE Transactions on Industrial Electronics, vol. 59, no. 5, pp. 2351-2362, May 2012.

[110] T. S. Ustun and R. H. Khan, "Multiterminal Hybrid Protection of Microgrids Over Wireless Communications Network," in IEEE Transactions on Smart Grid, vol. 6, no. 5, pp. 2493-2500, Sept. 2015.

[111] M. H. Cintuglu; T. Ma; O. Mohammed, "Protection of Autonomous Microgrids using Agent-Based Distributed Communication," in IEEE Transactions on Power Delivery, vol.PP, no.99, pp.1-1

[112] Brunet, Luc, Han-Lim Choi, and Jonathan P. How. "Consensus-based auction approaches for decentralized task assignment." AIAA Guidance, Navigation, and Control Conference, Honolulu, Hawaii. 2008.

[113] R. Olfati-Saber, J. A. Fax and R. M. Murray, "Consensus and Cooperation in Networked Multi-Agent Systems," in Proceedings of the IEEE, vol. 95, no. 1, pp. 215-233, Jan. 2007.

[114] Cidon, Isreal. "Yet another distributed depth-first-search algorithm."Information Processing Letters 26.6 (1988): 301-305.

[115] Makki, S. A. M., and George Havas. "Distributed algorithms for depth-first search." Information Processing Letters 60.1 (1996): 7-12.

[116] J. Chen and A. H. Sayed, "Diffusion Adaptation Strategies for Distributed Optimization and Learning Over Networks," in IEEE Transactions on Signal Processing, vol. 60, no. 8, pp. 4289-4305, Aug. 2012.

[117] M. H. Cintuglu, H. Martin and O. A. Mohammed, "Real-Time Implementation of Multiagent-Based Game Theory Reverse Auction Model for Microgrid Market Operation," in IEEE Transactions on Smart Grid, vol. 6, no. 2, pp. 1064-1072, March 2015.

[118] Simscape Power Systems., Available: http://www.mathworks.com/products/simpower/.

[119] Univ. Washington. Seattle, WA, Mar. 2006 [Online]. Available: http://www.ee.washington.edu/research/pstca./

[120] J. M. Guerrero, M. Chandorkar, T. L. Lee and P. C. Loh, "Advanced Control Architectures for Intelligent Microgrids-Part I: Decentralized and Hierarchical Control," in IEEE Transactions on Industrial Electronics, vol. 60, no. 4, pp. 1254-1262, April 2013. 
[121] J. M. Guerrero, P. C. Loh, T. L. Lee and M. Chandorkar, "Advanced Control Architectures for Intelligent Microgrids-Part II: Power Quality, Energy Storage, and AC/DC Microgrids," in IEEE Transactions on Industrial Electronics, vol. 60, no. 4, pp. 1263-1270, April 2013.

[122] J. M. Guerrero, J. C. Vasquez, J. Matas, L. G. de Vicuna and M. Castilla, "Hierarchical Control of Droop-Controlled AC and DC Microgrids-A General Approach Toward Standardization," inIEEE Transactions on Industrial Electronics, vol. 58, no. 1, pp. 158172, Jan. 2011.

[123] D. E. Olivares et al., "Trends in Microgrid Control," in IEEE Transactions on Smart Grid, vol. 5, no. 4, pp. 1905-1919, July 2014.

[124] H. S. V. S. Kumar Nunna and S. Doolla, "Multiagent-Based Distributed-EnergyResource Management for Intelligent Microgrids," in IEEE Transactions on Industrial Electronics, vol. 60, no. 4, pp. 1678-1687, April 2013.

[125] M. Mao, P. Jin, N. D. Hatziargyriou and L. Chang, "Multiagent-Based Hybrid Energy Management System for Microgrids," in IEEE Transactions on Sustainable Energy, vol. 5, no. 3, pp. 938-946, July 2014.

[126] Y. S. Foo. Eddy, H. B. Gooi and S. X. Chen, "Multi-Agent System for Distributed Management of Microgrids," in IEEE Transactions on Power Systems, vol. 30, no. 1, pp. 24-34, Jan. 2015.

[127] Communication Networks and Systems for Power Utility Automation for Distributed Energy Resources (DER) — Part 7-420, IEC61850, Int. Elec ᄀtrotech. Committee, 2011.

[128] Odell, James, and Marian Nodine. "The foundation for intelligent physical agents." Retrievable at: http://www. fipa.org (2006).

[129] Bellifemine, Fabio, Agostino Poggi, and Giovanni Rimassa. "JADE-A FIPA-compliant agent framework." Proceedings of PAAM. Vol. 99. No. 97-108. 1999.

[130] R. de Azevedo; M. H. Cintuglu; T. Ma; O. Mohammed, "Multi-Agent Based Optimal Microgrid Control Using Fully Distributed Diffusion Strategy," in IEEE Transactions on Smart Grid, vol.PP, no.99, pp.1-1

[131] Y. Xu and Z. Li, "Distributed Optimal Resource Management Based on the Consensus Algorithm in a Microgrid," in IEEE Transactions on Industrial Electronics, vol. 62, no. 4, pp. 2584-2592, April 2015.

[132] C. Li, M. Savaghebi, J. C. Vasquez and J. M. Guerrero, "Multiagent based distributed control for operation cost minimization of droop controlled AC microgrid using incremental cost consensus," Power Electronics and Applications (EPE'15 ECCEEurope), 2015 17th European Conference on, Geneva, 2015, pp. 1-9. 
[133] Wood, Allen J., and Bruce F. Wollenberg. Power generation, operation, and control. John Wiley \& Sons, 2012.

[134] Maiti, A.; Kist, A.A.; Maxwell, A.D., "Real-Time Remote Access Laboratory With Distributed and Modular Design," in Industrial Electronics, IEEE Transactions on , vol.62, no.6, pp.3607-3618, June 2015

[135] Gomes, L.; Bogosyan, S., "Current Trends in Remote Laboratories," in Industrial Electronics, IEEE Transactions on , vol.56, no.12, pp.4744-4756, Dec. 2009

[136] Bera, S.; Misra, S.; Rodrigues, J.J.P.C., "Cloud Computing Applications for Smart Grid: A Survey," in Parallel and Distributed Systems, IEEE Transactions on , vol.26, no.5, pp.1477-1494, May 12015

[137] Li Da Xu; Wu He; Shancang Li, "Internet of Things in Industries: A Survey," in Industrial Informatics, IEEE Transactions on , vol.10, no.4, pp.2233-2243, Nov. 2014

[138] Bitzer, B.; Gebretsadik, E.S., "Cloud computing framework for smart grid applications," in Power Engineering Conference (UPEC), 2013 48th International Universities' , vol., no., pp.1-5, 2-5 Sept. 2013

[139] Skkynet White Paper "What is a good approach to industrial SaaS?", Available: https://www.skkynet.com/assets.

[140] M. Uslar, M. Specht, S. Rohjans, J. Trefke, J. Gonzalez, The Common Information Model CIM. Springer-Verlag Berlin Heidelberg, 2012.

[141] S. Chowdhury, S.P. Chowdhury, and P. Crossley, Microgrids and Active Distribution Networks. The institution of Engineering and Technology, 2009.

[142] P. Wei, Y. Yan, Y. Ni, J. Yen, and F.F. Wu, "A decentralized approach for optimal wholesale cross-border trade planning using multi-agent technology," IEEE Transactions on Power Systems, vol. 16, pp. 833 -838, 2001.

[143] S. Kahrobaee, R.A. Rajabzadeh, L. Soh, and S. Asgarpoor, "A multiagent modeling and investigation of smart homes with power generation, storage and trading features," IEEE Transactions on Smart Grid, vol. 4, pp. 659 - 668, 2013

[144] A.J. Bagnall, and G.D. Smith, "A multiagent model of the UK market in electricity generation," IEEE Transactions on Evolutionary Computation, vol. 9, pp. 522 - 536, 2005

[145] D. Divenyi, and A.M. Dan, "Agent-based modeling of distributed generation in power system control," IEEE Transactions on Sustainable Energy, vol. 4, pp. 886 - 893, 2013 
[146] D.W Bunn, and F.S. Oliveira, "Agent-based simulation- an application to the new electricity trading arrangements of England and Wales," IEEE Transactions on Evolutionary Computation, vol. 5, pp. 493 - 503, 2001

[147] L.A. Wehinger, G. Hug-Glanzmann, M.D. Galus, and G. Andersson, "Modeling electricity wholesale markets with model predictive and profit maximizing agents," IEEE Transactions on Power Systems, vol. 28, pp. 868 -876, 2013.

[148] T. Logenthiran, D. Srinivasan, and A.M. Khambadkone, "Multi-agent system for energy resource scheduling of integrated microgrids in a distributed system," Electric Power Systems Research, vol. 81, pp. $138-148,2011$.

[149] S.D.J. McArthur, E.M. Davidson, V.M. Catterson, A.L. Dimeas, N.D. Hatziargyriou, F. Ponci, and T. Funabashi, "Multi-agent systems for power engineering applications-part I: concepts, approaches, and technical challenges," IEEE Transactions on Power Systems, vol. 22, pp. $1743-1752,2007$.

[150] S.D.J. McArthur, E.M. Davidson, V.M. Catterson, A.L. Dimeas, N.D. Hatziargyriou, F. Ponci, and T. Funabashi, "Multi-agent systems for power engineering applications-part II: technologies, standards, and tools for building multi-agent systems," IEEE Transactions on Power Systems, vol. 22, pp. 1753 -1759, 2007.

[151] P. Cozzi, Jade Assessing Reverse Auctions as a Policy Tool for Renewable Energy Deployment. The Center for International Environment \& Resource Policy, 2012.

[152] Z. Alibhai, W.A. Gruver, D.B. Kotak and D. Sabaz, "Distributed coordination of microgrids using bilateral contracts," IEEE International Conference on Man and Cybernetics, pp. $1990-1995,2004$.

[153] Bevrani, H.; Daneshfar, F.; Hiyama, T., "A New Intelligent Agent-Based AGC Design With Real-Time Application," Systems, Man, and Cybernetics, Part C: Applications and Reviews, IEEE Transactions on , vol.42, no.6, pp.994,1002, Nov. 2012

[154] Yuen, C.; Oudalov, A.; Timbus, A., "The Provision of Frequency Control Reserves From Multiple Microgrids," Industrial Electronics, IEEE Transactions on , vol.58, no.1, pp.173,183, Jan. 2011

[155] H. Chen, R. Ye, X. Wang and R. Lu, "Cooperative Control of Power System Load and Frequency by Using Differential Games," in IEEE Transactions on Control Systems Technology, vol. 23, no. 3, pp. 882-897, May 2015.

[156] Daneshfar, F.; Bevrani, H., "Load-frequency control: a GA-based multi-agent reinforcement learning," Generation, Transmission \& Distribution, IET , vol.4, no.1, pp.13,26, January 2010 
[157] A. Pahwa et al., "Goal-Based Holonic Multiagent System for Operation of Power Distribution Systems," in IEEE Transactions on Smart Grid, vol. 6, no. 5, pp. 2510-2518, Sept. 2015.

[158] Cintuglu, M.H.; Martin, H.; Mohammed, O.A., "Real-Time Implementation of Multiagent-Based Game Theory Reverse Auction Model for Microgrid Market Operation," Smart Grid, IEEE Transactions on , vol.6, no.2, pp.1064,1072, March 2015

[159] A.J. Bagnall, and G.D. Smith, "A multiagent model of the UK market in electricity generation," IEEE Transactions on Evolutionary Computation, vol. 9, pp. 522 - 536, 2005

[160] D. Divenyi, and A.M. Dan, “Agent-based modeling of distributed generation in power system control," IEEE Transactions on Sustainable Energy, vol. 4, pp. 886 - 893, 2013

[161] D.W Bunn, and F.S. Oliveira, "Agent-based simulation- an application to the new electricity trading arrangements of England and Wales," IEEE Transactions on Evolutionary Computation, vol. 5, pp. 493 - 503, 2001

[162] Zeng, Ming, et al. "An Incentivized Auction-Based Group-Selling Approach for Demand Response Management in V2G Systems." Industrial Informatics, IEEE Transactions on 11.6 (2015): 1554-1563.

[163] Ramachandran, B.; Srivastava, S.K.; Edrington, C.S.; Cartes, D.A., "An Intelligent Auction Scheme for Smart Grid Market Using a Hybrid Immune Algorithm," Industrial Electronics, IEEE Transactions on , vol.58, no.10, pp.4603,4612, Oct. 2011

[164] Wang, Yunpeng, et al. "A game-theoretic approach to energy trading in the smart grid." Smart Grid, IEEE Transactions on 5.3 (2014): 1439-1450.

[165] Ma, Jinghuan, et al. "Incentive mechanism for demand side management in smart grid using auction." Smart Grid, IEEE Transactions on 5.3 (2014): 1379-1388.

[166] Cassandra, Anthony R. "A survey of POMDP applications." Working Notes of AAAI 1998 Fall Symposium on Planning with Partially Observable Markov Decision Processes. 1998.

[167] Young, Steve, et al. "Pomdp-based statistical spoken dialog systems: A review." Proceedings of the IEEE 101.5 (2013): 1160-1179.

[168] Ross, Stéphane, et al. "Online planning algorithms for POMDPs." Journal of Artificial Intelligence Research (2008): 663-704.

[169] Aiken, Bob, et al. "Report of nsf workshop on network research testbeds."National Science Foundation Directorate for Computer and Information Science and Engineering (CISE) Advanced Networking Infrastructure \& Research Division (2002). 
[170] Genge, B.; Beres, A.; Haller, P., "A survey on cloud-based software platforms to implement secure smart grids," in Power Engineering Conference (UPEC), 2014 49th International Universities, vol., no., pp.1-6, 2-5 Sept. 2014

[171] Jianli Pan; Jain, R.; Paul, S., "A Survey of Energy Efficiency in Buildings and Microgrids using Networking Technologies," in Communications Surveys \& Tutorials, IEEE , vol.16, no.3, pp.1709-1731, Third Quarter 2014

[172] Simmhan, Y.; Aman, S.; Kumbhare, A.; Rongyang Liu; Stevens, S.; Qunzhi Zhou; Prasanna, V., "Cloud-Based Software Platform for Big Data Analytics in Smart Grids," in Computing in Science \& Engineering, vol.15, no.4, pp.38-47, July-Aug. 2013

[173] Rusitschka, S.; Eger, K.; Gerdes, C., "Smart Grid Data Cloud: A Model for Utilizing Cloud Computing in the Smart Grid Domain," in Smart Grid Communications (SmartGridComm), 2010 First IEEE International Conference on, vol., no., pp.483488, 4-6 Oct. 2010

[174] Maheshwari, K.; Lim, M.; Wang, L.; Birman, K.; van Renesse, R., "Toward a reliable, secure and fault tolerant smart grid state estimation in the cloud," in Innovative Smart Grid Technologies (ISGT), 2013 IEEE PES, vol., no., pp.1-6, 24-27 Feb. 2013

[175] Mohsenian-Rad, A.-H.; Leon-Garcia, A., "Coordination of Cloud Computing and Smart Power Grids," in Smart Grid Communications (SmartGridComm), 2010 First IEEE International Conference on, vol., no., pp.368-372, 4-6 Oct. 2010

[176] Vukovic, O.; Dan, G.; Bobba, R.B., "Confidentiality-preserving obfuscation for cloudbased power system contingency analysis," in Smart Grid Communications (SmartGridComm), 2013 IEEE International Conference on, vol., no., pp.432-437, 2124 Oct. 2013

[177] Xi Fang; Dejun Yang; Guoliang Xue, "Evolving Smart Grid Information Management Cloudward: A Cloud Optimization Perspective," in Smart Grid, IEEE Transactions on , vol.4, no.1, pp.111-119, March 2013 
VITA

\section{MEHMET HAZAR CINTUGLU}

1986

2004-2008

2008-2011

$2012-2016$

2015
Born, Adana, Turkey

B.S., Electrical Engineering

Gazi University, Ankara, Turkey

M.S., Electrical Engineering

Gazi University, Ankara, Turkey

Doctoral Candidate, Electrical Engineering

Florida International University, Miami, Florida

Award, Dissertation Year Fellowship

Florida International University, Miami, Florida

\section{SELECTED PUBLICATIONS AND PRESENTATIONS}

[J-1] M. H. Cintuglu; O. A. Mohammed, "Behavior Modeling and Auction Architecture of Networked Microgrids for Frequency Support," in IEEE Transactions on Industrial Informatics, vol.PP, no.99, pp.1-1

[J-2] R. de Azevedo; M. H. Cintuglu; T. Ma; O. Mohammed, "Multi-Agent Based Optimal Microgrid Control Using Fully Distributed Diffusion Strategy," in IEEE Transactions on Smart Grid, vol.PP, no.99, pp.1-1

[J-3] M. H. Cintuglu; T. Youssef; O. A. Mohammed, "Development and Application of a Real-Time Testbed for Multiagent System Interoperability: A Case Study on Hierarchical Microgrid Control," in IEEE Transactions on Smart Grid, vol.PP, no.99, pp.1-1

[J-4] M. H. Cintuglu; T. Ma; O. Mohammed, "Protection of Autonomous Microgrids using Agent-Based Distributed Communication," in IEEE Transactions on Power Delivery, vol.PP, no.99, pp.1-1

[J-5] M. H. Cintuglu, H. Martin and O. Mohammed, "Real-Time Implementation of Multiagent-Based Game Theory Reverse Auction Model for Microgrid Market Operation,” IEEE Trans. Smart Grid, vol.6, no.2, pp.1064-1072, March 2015. 
[J-6] A. Abdulaal; M. H. Cintuglu; S. Asfour; O. Mohammed, "Solving the MultiVariant EV Routing Problem Incorporating V2G and G2V Options," in IEEE Transactions on Transportation Electrification, vol.PP, no.99, pp.1-1

[J-7] T. Ma; M. H. Cintuglu; O. A. Mohammed, "Control of Hybrid AC/DC Microgrid Involving Storage, Renewable Energy and Pulsed Loads," in IEEE Transactions on Industry Applications, vol.PP, no.99, pp.1-1

[J-8] M. H. Cintuglu; O. A. Mohammed; K. Akkaya; A. S. Uluagac, "A Survey on Smart Grid Cyber-Physical System Testbeds," in IEEE Communications Surveys \& Tutorials, vol.PP, no.99, pp.1-1

[C-1] M. H. Cintuglu, A. T. Elsayed and Osama Mohammed, "Microgrid automation assisted by synchrophasors," Smart Grid Technologies Conference (ISGT), 2015 IEEE Power \& Energy Society, Washington, DC, 2015, pp. 1-5.

[C-2] M. H. Cintuglu, T. A. Youssef, A. T. Elsayed and O. A. Mohammed, "Frequency and voltage control of microgrids upon unintentional cascading islanding," SoutheastCon 2015, Fort Lauderdale, FL, 2015, pp. 1-6.

[C-3] M. H. Cintuglu and O. A. Mohammed, "Simulation of digitalized power system using PMU and intelligent control,"Industry Applications Society Annual Meeting, 2013 IEEE, Lake Buena Vista, FL, 2013, pp. 1-8.

[C-4] A. Aydeger, K. Akkaya, M. H. Cintuglu, A. S. Uluagac and O. Mohammed, "Software defined networking for resilient communications in Smart Grid active distribution networks," 2016 IEEE International Conference on Communications (ICC), Kuala Lumpur, 2016, pp. 1-6.

[C-5] A. Mazloomzadeh, M. H. Cintuglu and O. A. Mohammed, "Islanding detection using synchronized measurement in smart microgrids," Innovative Smart Grid Technologies Latin America (ISGT LA), 2013 IEEE PES Conference On, Sao Paulo, 2013, pp. 1-7.

[C-6] M. H. Cintuglu, H. Martin and O. A. Mohammed, "An intelligent multi agent framework for active distribution networks based on IEC 61850 and FIPA standards," Intelligent System Application to Power Systems (ISAP), 2015 18th International Conference on, Porto, 2015, pp. 1-6.

[C-7] M. H. Cintuglu, Tan Ma and O. A. Mohammed, "Aggregated active distribution networks for secondary control of islanded power systems," 2015 IEEE Power \& Energy Society General Meeting, Denver, CO, 2015, pp. 1-5. 